\author{
Universidade de São Paulo \\ Instituto de Física
}

\title{
Fenomenologia de Teorias com um Higgs Composto
}

\author{
Pedro Bittar
}

Orientador: Prof. Dr. Gustavo Burdman

Dissertação de mestrado apresentada ao Instituto de Física da Universidade de São Paulo, como requisito parcial para a obtenção do título de Mestre em Ciências.

Banca Examinadora:

Prof. Dr. Gustavo Burdman - Orientador FMA/IFUSP

Prof. Dr. Rogério Rosenfeld - IFT/UNESP

Prof. Dr. Pedro Galli Mercadante - UFABC 
FICHA CATALOGRÁFICA

Preparada pelo Serviço de Biblioteca e Informação do Instituto de Física da Universidade de São Paulo

\section{Souza, Pedro Bittar Oliveira}

Fenomenologia de teorias com um Higgs composto / On the phenomenology of theories with a composite Higgs. São Paulo, 2020.

Dissertação (Mestrado) - Universidade de São Paulo. Instituto de Física. Depto. de Física Matemática

Orientador: Prof. Dr. Gustavo Burdman Campos.

Área de Concentração: Física de Partículas Elementares e

Unitermos: 1. Teoria quântica de campo; 2. Teoria de campos;

3. Física teórica. 


\title{
University of São Paulo \\ Institute of Physics
}

\section{On the Phenomenology of Theories with a Composite Higgs}

\author{
Pedro Bittar
}

Supervisor: Prof. Dr. Gustavo Burdman

Dissertation submitted to the Physics Institute of the University of São Paulo in partial fulfillment of the requirements for the degree of Master of Science.

Examining Committee:

Prof. Dr. Gustavo Burdman - Supervisor FMA/IFUSP

Prof. Dr. Rogério Rosenfeld - IFT/UNESP

Prof. Dr. Pedro Galli Mercadante - UFABC 



\section{Agradecimentos}

Primeiramente gostaria de agradecer ao meu orientador, Gustavo Burdman. Sou grato pela sua confiança em colocar esse projeto nas minhas mãos e pela liberdade e segurança garantida para seguir os meus próprios caminhos. Também agradeço ao Dorival Gonçalves pela quase co-orientação e enorme ajuda em tempos nebulosos. Ao Massoni pelas várias discussões sobre o destino da física de partículas e colegas do júnior club e aos professores do departamento.

Agradeço ao Conselho Nacional de Desenvolvimento Científico e Tecnológico (CNPq) pelo auxílio financeiro.

À todos que me apoiaram ao longo dessa trajetória. Meus amigos e familiares de São João del Rei, que por estarem tão longe, fazem da minha estadia em São Paulo um pouco mais difícil. Principalmente, gostaria de agradecer aos meus avós Ivan, Lourdes, Vera e Fred pelo carinho e ajuda ao longo de todos esses anos. Ao meu pai que me ensinou a melhor qualidade dele - a ambição. Obrigado por sempre buscar ficar por perto. E ao meu irmão que sempre me lembra que não devo levar tudo tão à sério.

Agora, às pessoas que fizeram a minha estadia em São Paulo mais feliz. Obrigado aos Wiladores, pelas discussões e tempo perdido no café. À família da Isa pela recepção e goiabinhas. Finalmente, à Isa que segurou a minha mão nos momentos de surto e delírio que passei para chegar aqui. Não teria conseguido sem o carinho dela.

Minha vida acadêmica só foi possível graças a minha mãe. Dedico tudo que escrevo, e que sou, à ela.

"Science changed drastically...

from trying to show that the world is intelligible to us, which was given up, to trying to show that we can construct theories that are intelligible to us."

Noam Chomsky 



\title{
UNIVERSITY OF SÃO PAULO
}

\author{
Abstract \\ IFUSP \\ Mathematical Physics Department \\ Master's degree

\section{On the Phenomenology of Theories with a Composite Higgs}

by Pedro Bittar

The Standard Model (SM) is a complete and well-tested framework for describing elementary particles interactions. However, when discussing the stability of the Electroweak (EW) scale, it lacks theoretical grounding. More concretely, having a light Higgs boson is not naturally attainable if there is new microscopic physics above the EW scale. This issue is the hierarchy problem (HP)

The Higgs boson is the agent of Electroweak Symmetry Breaking (EWSB), the origin of particles masses. Here, if the symmetry structure of the SM is maintained, the elementary nature of the Higgs inevitably introduces the HP. Even though the SM parametrizes the Higgs mechanism, it offers no dynamical explanation for EWSB and, consequently, causes the HP. Giving a composite nature to the Higgs can solve such a problem, while also providing EWSB with new dynamical content.

Such a proposal is at the core of composite Higgs models (CHM). They provide a general framework for extending the $\mathrm{SM}$ at the $\mathrm{TeV}$ scale, the aimed energy scales of the LHC, and to attack the HP. However, when it comes to the experimental signatures of compositeness itself, such models may lack in their effective local descriptions.

Our objective is to look for general signs of compositeness in collider experiments. The task ahead is to find a systematic way of including compositeness effects while providing the means to study its phenomenology. Throughout our work, we have restored the full momentum dependence of compositeness via the form factor formalism and studied its phenomenological consequences through simulations. Here, we discovered changes in the shapes of kinematic distributions, that are typical of compositeness. If the Higgs is composite, such signatures should start to become apparent in the next stages of the LHC experiment.

Keywords: Composite Higgs Models, Form Factor, Momentum Dependence, Hierarchy Problem, Standard Model 



\title{
UNIVERSIDADE DE SÃO PAULO
}

\author{
Resumo \\ IFUSP \\ Departamento de Física Matemática
}

Tese de Mestrado

\section{Fenomenologia de Teorias com um Higgs Composto}

por Pedro Bittar

O Modelo Padrão (MP) é uma descrição completa e bem testada das interações de partículas elementares. Contudo, quando se discute a estabilidade da escala eletrofraca (EW), a sua estrutura parece incompleta. Concretamente, ter um boson de Higgs leve não é atingível de maneira natural se existe física microscópica além da escala EW. Essa questão é o problema da hierarchia $(\mathrm{PH})$.

O boson de Higgs é o agente da quebra espontânea da simetria eletrofraca (EWSB), responsável pela origem das massas das paticulas elementares. Aqui, se a estrutura de simetrias do MP for mantida, o caráter elementar do Higgs vai, inevitavelmente, reintroduzir o PH. Embora o MP parametrize o mecanismo de Higgs, ele não oferece uma explicação dinâmica para EWSB, e consequentemente, causa o PH. Dar uma natureza composta para o Higgs pode resolver o PH e também dar um novo conteúdo dinâmico para EWSB.

Essa proposta está no centro dos modelos do Higgs composto. Eles dão um contexto geral para extender o MP na escala dos $T e V$ e atacam diretamente o PH. Contudo, no que tange ao sinais experimentais da composição do Higgs, esses modelos podem falhar na sua descrição efetiva e local.

Nosso objetivo é olhar para os sinais mais gerais do Higgs composto nos experimentos de colisor. A tarefa é encontrar uma maneira sistematica de incluir os efeitos de uma partícula composta na teoria e derivar meios para estudar a sua fenomenologia. No nosso trabalho, tivemos que restaurar completamente a dependência no momento de um Higgs composto, e estudamos os sinais desse caso por simulações. Aqui, encontramos modificações nas distribuições cinemáticas, que são típicas do cenário assumido. Se o Higgs é de fato composto, os seus efeitos devem começar a ser visíveis nas próximas fases do LHC.

Palavras-Chave: Higgs Composto, Fator de Forma, Dependência com o momento, Problema da Hierarquia, Modelo Padrão. 



\section{Contents}

$\begin{array}{ll}\text { Agradecimentos } & \text { iii }\end{array}$

$\begin{array}{lll}\text { Abstract } & \text { v }\end{array}$

Resumo vii

1 Introduction 1

1.1 Elementary and Composite Particle Physics . . . . . . . . . . . . . 3

1.2 The Compositeness of the Higgs Boson . . . . . . . . . . . . . . . . 18

2 A Composite Higgs Boson $\quad 23$

2.1 The pseudo-Nambu-Goldstone Higgs Particle . . . . . . . . . . . . . . 27

2.1.1 A New Composite Sector . . . . . . . . . . . . . . . . 30

2.1.2 Embeeding the Standard Model . . . . . . . . . . . . . . 33

2.1.3 Vacuum Misalignment . . . . . . . . . . . . 36

2.2 Non-linearly Realized Symmetries . . . . . . . . . . . . . . . . . 38

2.3 Composite Higgs couplings in the MCHM5 . . . . . . . . . . . . . . . 41

2.3.1 Gauge Boson Interactions . . . . . . . . . . . . . . . 43

2.3.2 Fermion Interactions . . . . . . . . . . . . . . . 45

2.4 Interactions between Elementary and Composite Sectors . . . . . . . 48

2.4.1 Composite and Elementary Mixing . . . . . . . . . . . . . . 48

2.4.2 The Composite Hierarchy Solution . . . . . . . . . . . . . 52

3 Strong Dynamics via Nonpertubative methods $\quad 57$

3.1 Spectral Properties . . . . . . . . . . . . . . . . 58

3.1.1 The Spectral Decomposition . . . . . . . . . . . 63

3.2 The $1 /$ N Expansion . . . . . . . . . . . . . . . . . . . 66

3.3 Tools for Form Factor Model-Building . . . . . . . . . . . . . . . . 70

3.3.1 Narrow Resonances Limit . . . . . . . . . . . . . . . . . 70

3.3.2 Weinberg Sum Rules . . . . . . . . . . . . . . . . 72

3.3.3 Form Factors . . . . . . . . . . . . . . . . . . . 75

3.4 Effective Field Theory Matching . . . . . . . . . . . . . . 76

3.5 Higher Point Functions . . . . . . . . . . . . . . . . . . . . . . . . 78 
4 Composite Higgs Form Factors $\quad \mathbf{8 1}$

4.1 Simple Form Factor Ansatzes . . . . . . . . . . . . . . . . . . . . . 82

4.1 .1 Two-Point Form Factors . . . . . . . . . . . . . . . . . 83

4.1.2 Three-Point Form Factors . . . . . . . . . . . . . . . . 88

4.2 The $\mathrm{MCHM}_{5}$ Form Factors . . . . . . . . . . . . . . . . . . . . . . 92

4.2.1 Fermion Form Factor . . . . . . . . . . . . . . . . . . . 93

Couplings to the Higgs . . . . . . . . . . . . . . . . . 99

Matching with the EFT . . . . . . . . . . . . . . . . . . . . . . . . . . . . . . . .

4.2.2 Gauge Bosons Form Factor . . . . . . . . . . . . . . . . . . . 106

Electroweak Precision Tests . . . . . . . . . . . . 109

4.3 Overview of the Form Factor Formalism . . . . . . . . . . . . . . . 112

5 Collider Phenomenology of Form Factors 113

5.1 Zero Momentum Phenomenology of CHMs . . . . . . . . . . . . . . 117

5.2 Form Factor Phenomenology . . . . . . . . . . . . . . . . . . 120

5.2 .1 Numerical Implementation . . . . . . . . . . . . . . . . . . . 121

5.2 .2 The tth Channel . . . . . . . . . . . . . . . . . 124

Theory Constraints . . . . . . . . . . . . . . 125

Results . . . . . . . . . . . . . 126

5.2 .3 The ggh Channel . . . . . . . . . . . . . . . . . . 134

Higgs Off-Shell . . . . . . . . . . . . . . 135

Modification of the Loop Integral . . . . . . . . . . . . 137

5.2.4 Other Channels . . . . . . . . . . . . . . . . . 139

5.3 Compositeness from the Collider Perspective . . . . . . . . . . . . 140

6 Discussions and Conclusion $\quad 141$

6.1 The Composite Higgs Picture . . . . . . . . . . . . . . . . . . . . . . 142

6.2 Perspectives . . . . . . . . . . . . . . . . . . . . . . 145

6.3 Conclusion . . . . . . . . . . . . . . . . . . . . 153

$\begin{array}{ll}\text { References } & 155\end{array}$ 


\section{Chapter 1}

\section{Introduction}

A large share of the particle physics community is currently dedicated to studying the Higgs boson interactions at the Large Hadron Collider (LHC). The last decades of theoretical endeavours suggested that there should be deviations from the current framework onset, the Standard Model (SM). Furthermore, it is suggested that Electroweak Symmetry Breaking (EWSB), the origin of all the SM particle masses, is incomplete. The expectation is that these new effects should be accessible at the current LHC TeV scale. Altogether, the Higgs [1] seems to be the central piece of this puzzle.

However, it has been a remarkable task to include beyond the standard model (BSM) effects into the current framework. The main difficulty is the so-called Hierarchy Problem (HP), which reflects the trouble in conciliating the elementary Higgs $125 \mathrm{GeV}$ mass with the scale of new physics. Problematically, quantum corrections to the Higgs mass term suggest that it should be much heavier.

In all of this, the Higgs is assumed to have the same status of the remaining SM particles - it is elementary. The Higgs of the SM has no internal structure or available symmetry to protect its mass from these large quantum corrections. Thus, we are bound to the HP by the very nature of this SM scalar particle. It was against these difficulties that composite Higgs Models (CHM) were proposed.

CHMs were built to provide a solution to the HP. Moreover, they are local effective field theories (EFT's) that modify the SM coupling constants. Importantly, these couplings shifts do not bear any distinctive features of composite structures since they cannot be distinguished from elementary ones. 
As a result, CHMs effective descriptions are not enough to describe the full content of a composite Higgs. Surely, we expect that compositeness should have its own phenomenological signals. The question, above all, is about how to explore the full extent of compositeness when we are limited to LHC scale experiments.

Thus, the purpose of this work is to investigate the general signatures of the Higgs compositeness in upcoming collider experiments.

Hierarchy in nature's scales has been a central concept throughout the development of physics. A remarkable feature of the particle physics framework is that hierarchy is built-in the construction of our models as we explore experimentally from small energies to higher ones. Historically, much has happened in this program to extend the high energy frontier, since when a new energy benchmark is reached, we often force ourselves to redefine the labels of the elementary constituents of our models. One can say that looking for the next energy benchmark is the program for the current generation of physicists. Undoubtedly, the theoretical puzzles we are facing have paramount importance to such a goal.

To some degree, we know the relationship between the full range of observed phenomena up until the electroweak scale, which defines the realm of the SM. The way it is, the SM is the theory of the Electroweak scale. Exploring it was the driving force of past and current collider experiments. At this point, the thoughtto-be program of 2010's physics was to discover the Higgs and whichever new physics that could come along.

Coming as a shock, the absolute experimental adequacy of the SM forces an unnatural behaviour when incorporating scales beyond the ones characterized by the Higgs mass. Moreover, the HP was treated as the expectation of a new energy benchmark associated with the Higgs. At this new scale, hints of a completion for the 
SM should become evident, with the appearance of new particles and interactions ${ }^{1}$. The shock is that only the Higgs came up, and nothing else so far. Not only we discovered the Higgs, but it behaves just as it should in the SM.

By trying to extend the theory to include new massive phenomena, we discover that the SM suffers this unexplainable amount of fine-tuning. Keeping the next new physics threshold separated from the electroweak scale creates this hierarchy, which requires a fine parameter calibration. Such puzzle suggests the Higgs sector has more structure than what was foreseen by the SM. This tension is at the core of the Higgs theoretical developments today, and the appearance of the next energy benchmark still seems to be unwarranted.

Even with proposed solutions, hierarchy and elementarity are two concepts that are hard to conciliate. Many different solutions to the HP that keep the elementary status of the Higgs face phenomenological challenges today. By giving up particle elementarity, we are putting the Higgs at the edge of our knowledge, and predicting the appearance of new phenomenology at the $T e V$ scale. We are assuming that the Higgs emerges from a yet undiscovered sector, Beyond the Standard Model (BSM) [47, 46].

So far the HP has served as an essential guide for BSM. However, this work is primarily concerned with the Higgs status as a composite. The attitude here is to postpone the conflict of the $\mathrm{TeV}$ scale, shifting from the HP to the consequences of a composite Higgs boson. How different is a composite Higgs from an elementary one?

\subsection{Elementary and Composite Particle Physics}

The situation though the 1920s was that the electron and proton were considered the fundamental constituents of matter. Eventually, the composite nature of nucleons was bound to emerge as experimental technology further sophisticated.

\footnotetext{
${ }^{1}$ Like supersymmetry, extra dimensions, and even composite higgses
} 
Interestingly, such dynamics underlined much of the advances in particle physics [17].

It was in the 1950s that the extended composite nature of nucleons started to become apparent through charge distribution measurements.

Through these experiments, the crucial characteristics of compositeness were manifest as the momentum transfer allowed a probe to the internal structure of particles. Here, the momentum dependence posed modifications to the distribution functions of elementary particles, and this was the main signal of a composite object - shapes of cross-sections distributions do distinguish elementarity from compositeness.

As an example we can look for the the expected differential cross section of the elementary electron scattering, predicted by the Mott formula. ${ }^{2}$

$$
\left(\frac{d \sigma}{d \Omega}\right)_{\mathrm{Mott}}=\frac{\alpha^{2}}{4 E^{2} \sin ^{4} \theta / 2} \cos ^{2} \frac{\theta}{2}
$$

The Mott cross-section is for structureless scattering. Here the target proton recoil and size are neglected together with the magnetic spin-spin interactions. To take into account the finite size of the proton, reflected through a certain charge distribution, the Mott cross section gets modified by a Form Factor.

$$
\frac{d \sigma}{d \Omega}=\left(\frac{d \sigma}{d \Omega}\right)_{\mathrm{Mott}}\left|F\left(q^{2}\right)\right|^{2}
$$

where the vector $q$ is defined as the momentum transfer of the process, and the form factor $F\left(q^{2}\right)$ is the Fourier transform of the charge distribution.

A form factor reflects the finite size of the scattering center and induces a phase difference between the electron plane waves. If the incoming wavelength is very large compared to the proton's size, it is reduced to $F\left(q^{2}\right)=1$. In such case,

\footnotetext{
${ }^{2}$ Here $\alpha$ is the fine-structure constant, $E$ is the electron beam energy and $\theta$ is the relative angle between incoming and outgoing electrons scattering over a fixed proton.
} 
the elementary behaviour is recovered. This limit serves as a normalization, and at zero transferred momentum, the effects of compositeness disappear.

Another essential point is that the transferred momentum has a kinematic dependence associated with it. This dependence is the main feature of the extended character of the proton - deviations from point-like behaviour induce modifications in the differential kinematic distributions. This behaviour can be seen in the crosssections measurements of electron scattering from protons, as in figure 1.1.

In short, the important fea-

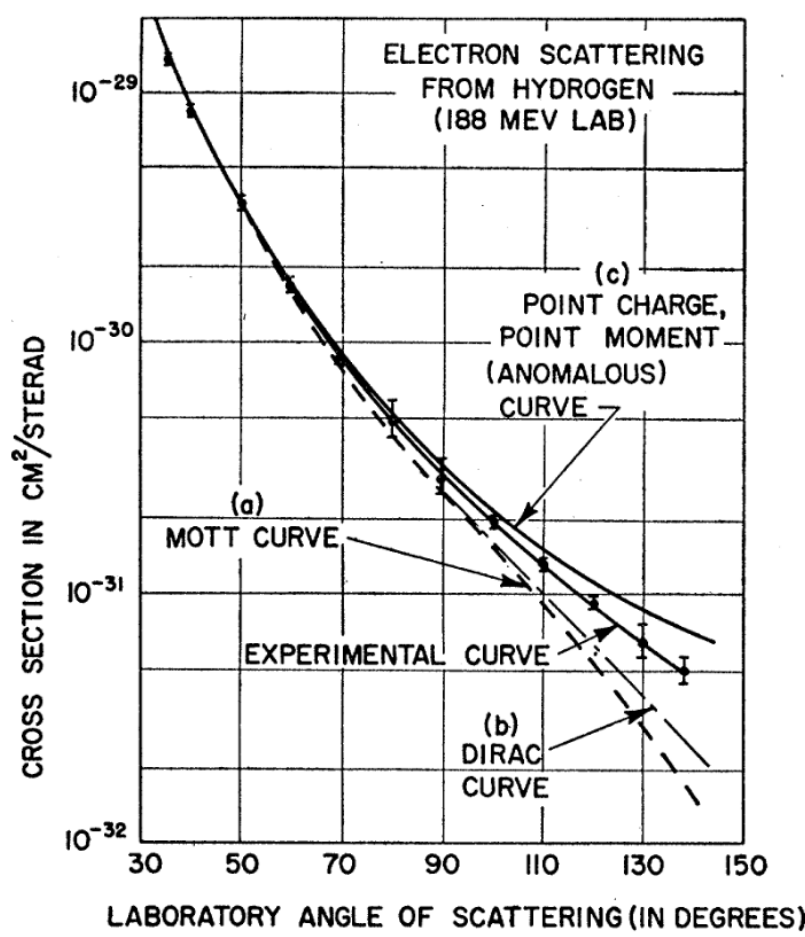

FiguRE 1.1: Electron scattering from the proton at incident energy of $188 \mathrm{MeV}$ [49]. The shape of the distribution tail deviats from the elementary prediction due to proton compositeness. tures of compositeness are observed when there is enough momentum flow to access the extended structure. When accessed, a composite object induce modifications to the point-like kinematic distributions.

Before the charge distribution measurements, the first evidence for a composite structure of nucleons came with the magnetic moment measurements performed by Otto Stern [55, 56]. The Dirac equation predicts that the magnetic moment is $g=2$ for free elementary fermions. The striking discovery was that the proton and neutron magnetic moments depart significantly from the predicted value for structureless elementary fermions. Such shifts in particle couplings indicate composite behaviour, as shown in table 1.1.

The electron's g-factor also departs slightly from the exact $g=2$ value, but it is still expected to be elementary. The reason is not related to coupling shifts but 
due to the absence of internal constituents. Even though there is a cloud of virtual particles surrounding the electron, these are not physical states and do not suggest a meaningful sign of compositeness at low energies.

\begin{tabular}{|l|l|l|}
\hline Particle & Symbol & g-factor \\
\hline Dirac & $g$ & 2 \\
Electron & $g_{e}$ & $2.00231930436256(35)$ \\
Muon & $g_{\mu}$ & $2.0023318418(13)$ \\
Neutron & $g_{n}$ & $3.82608545(90)$ \\
Proton & $g_{p}$ & $5.5856946893(16)$ \\
\hline
\end{tabular}

TABlE 1.1: Magnetic moments of different particles. Electrons and muons are closer to the Dirac value. For the neutron and proton, the departure from $g=2$ are due to their internal structure.

Actually, electrons could be composite in very high scales. The effective running of $\alpha$ suggests the presence of a Landau pole. At high energies, a gauge $U(1)$ becomes a nonperturbative theory and is not asymptotically free. It is said that the theory has such a Landau pole when the coupling becomes infinite at finite energy. Such behaviour is often attributed as a sign of theoretical incompleteness and could be attributed to the emergence of a complete theory at the Landau pole scale.

However the strong coupling limit of the SM is at astonishingly high energies, $\sim 10^{286} \mathrm{GeV}$. Even though the actual electrons constituents might arise in a complete theory, it is not sensible in any way to look for electrons compositeness signs since they are very far away. For all senses, electrons remain elementary particles.

Such discussion introduces a crucial aspect of compositeness - it is a scaledependent concept.

A much more accessible example of nonperturbative behaviour occurs in Quantum Chromodynamics (QCD). There, the perturbativity breakdown occurs in the low energy infrared (IR) region instead of the ultraviolet (UV). QCD at such scale is responsible for the emergence of hadrons.

The first attempts to build a hadronic Lagrangian was particles such as pions, nucleons, sigmas and rhos. In it, pions are naturally light compared to the much more massive nucleons and others. Such property is the result of them being 
the pseudo-Nambu-Goldstone bosons of chiral symmetry breaking. This symmetry structure is deeply rooted in the spontaneous symmetry breaking (SSB) of chiral symmetry and will be mimicked when we proceed to make the Higgs a composite scalar later on.

By looking closer into interactions, we can start to access the composite sector via the momentum transfer of interactions. This aspect became most apparent in the pion electromagnetic form factor discussion, in which yet another hadronic resonance appears - the $\rho$-meson vector.

Form factors encapsulate the contributions of heavier intermediate particles to physical processes. They do it without having to explicitly assign fields for these states, thus making them a non-local approach.

The electromagnetic form factor $G_{\pi}\left(q^{2}\right)$ of charged pions can be defined as the matrix element of the electromagnetic current between two pion states. Lorentz and Gauge invariance force this element to be of the form:

$$
\left\langle\pi^{+}\left(\mathbf{p}_{2}\right)\left|J_{e m}^{\mu}\right| \pi^{+}\left(\mathbf{p}_{1}\right)\right\rangle=G_{\pi}\left(q^{2}\right)\left(p_{1}+p_{2}\right)^{\mu}
$$

with $q^{\mu}=\left(p_{1}-p_{2}\right)^{\mu}$ is the transfered momentum to the photon. Physically, the interaction is dominated by a mixing of the photon and the $\rho$ resonance. Such interaction is diagonalized at zero tranfered momentum, making $G_{\pi}(0)=1$ to ensure gauge invariance. The mixing must occur at large enough momentum transfer $q^{2}$ to be kinematically effective as $G_{\pi}(0)=1$ is

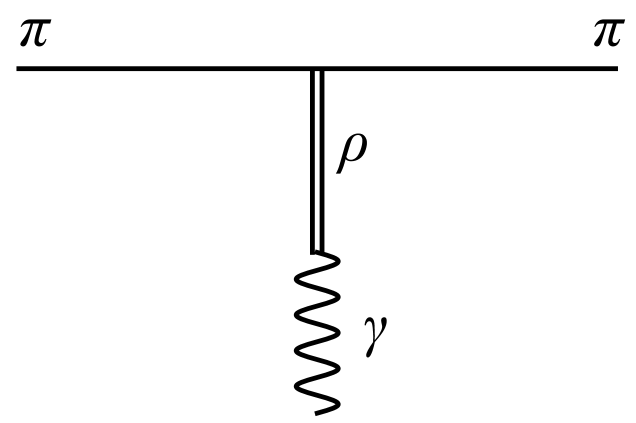

Figure 1.2: The pion form factor under the one vector-meson dominance hyphothesis. forced by the masslessness of the photon. 
The use of mixing interactions to describe the form factor became known as the vector-meson dominance hypothesis in the context of Hidden local symmetries $[53,54]$. Phenomenologically, they provided a good description of the pion form factor since the $\rho$ 's were the lightest hadrons that participate in such a process. Diagrammatically, we can see in figure 1.2 how the $\rho$ dominates the pion process.

The form factor expression in such approximation is given by a monopole term, which reflects the intermediate $\rho$ state between the pions and the photon.

$$
G_{\pi}\left(q^{2}\right) \simeq \frac{1}{1-q^{2} / m_{\rho}^{2}}, \quad m_{\rho} \approx 775 \mathrm{MeV}
$$

Eventually, the advance in experimental techniques permitted the discovery of the Hadron zoo, with hundreds of new particles in the $G e V$ range. This scale defines the order parameter in which the fundamental degrees of freedom confines to form composite hadrons. At the time, making sense of such a theory was incredibly hard, since the interaction became strong enough to surpass the perturbativity limit.

Assigning a field for each new state was a pointless strategy. It was unprecedented the necessity of formulating a nonperturbative approach to QFT, which was before the emergence of the fundamental QCD theory. Many alternatives to the Quantum Field Theory scheme were proposed for dealing with these strongly interacting theories and, eventually, all of them became incorporated into QFT's toolkit. Some examples are the Bootstrap models and techniques such as spectral decomposition, large-N limit and sum rules. Surprisingly, these tools will be useful in upcoming discussions of the Higgs compositeness here.

Despite the necessity to develop nonperturbative tools, the discovery of quarks, gluons and the asymptotically free structure of QCD postponed this challenge. After 1973, the necessity to understand QCD properties at high energies drove the particle physics field, and the alternatives were mainly abandoned. Perturbative calculations were then reliable in the high energy limit, and elementarity emerged again. In figure 1.3, we show the evolution of $\alpha_{s}$ that indicate the asymptotically free behaviour of 


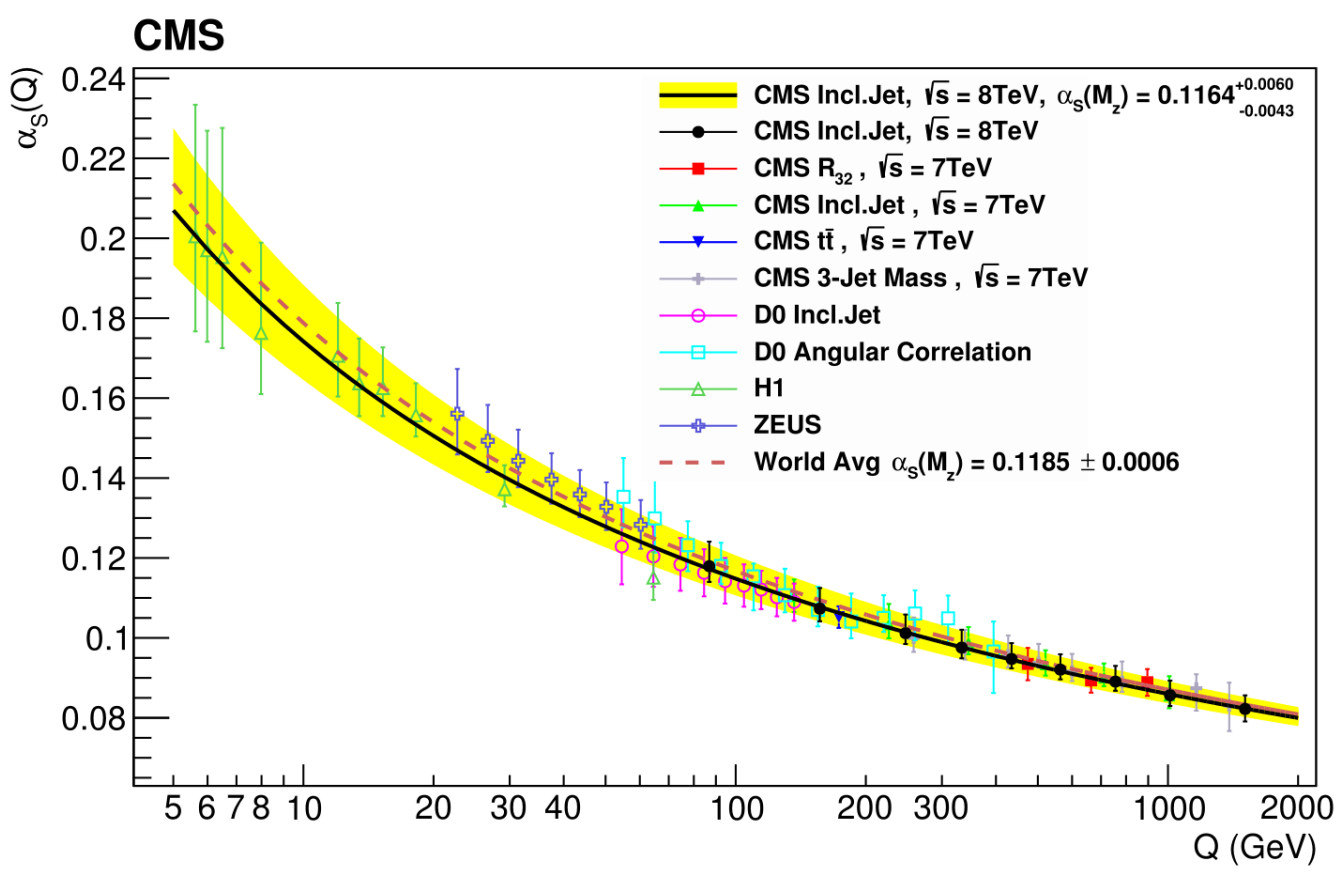

Figure 1.3: Measurements of the strong coulpling of QCD as a function of the transferred momentum $Q$. Taken from [50].

QCD.

In QCD, the combination of six elementary $S U(3)$ charged fermions and their interaction pattern with new gauge bosons was responsible for forming hadrons at low energies as bound states. This happens because of confinement - a truly nonperturbative effect.

In light of $\mathrm{QCD}$, the compositeness of hadrons became a clear subject. Hadrons are bound states of quarks and gluons that can be accessed in collider experiments. Traditionally, the most important process used to probe the structures inside the hadrons was Deep Inelastic Scattering (DIS) [62, 63, 64].

DIS provided experimental evidence for the existence of an internal structure of hadrons. It pointed to the existence of three valence quarks forming the baryons and two forming mesons. Ultimately, it set the elementary status of quarks.

After the typical hadronic energies, at $E>>\Lambda_{Q C D}$, the internal constituents started to become clearly visible to detectors. At colliders, these effects were seen 


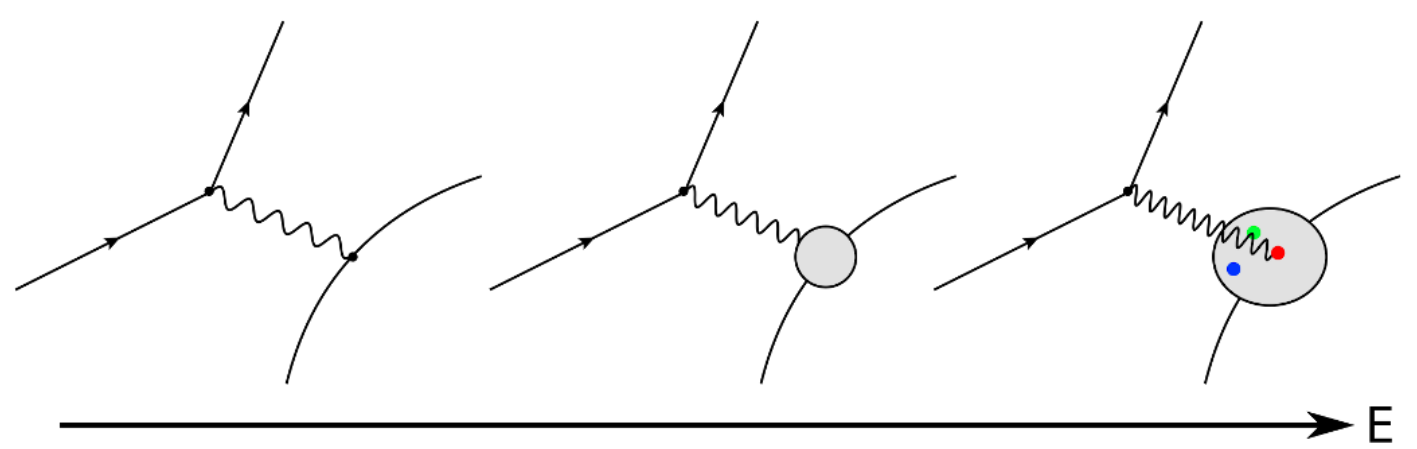

Figure 1.4: Experimental probe to a composite object. From a seemingly elementary interaction (left), to a form factor type of interaction (middle) until the experimental resolution is enought to probe internal constituents (right).

as jets, which are the result of the production of quarks and gluons. Subsequently, they hadronize producing very distinctive trails on calorimeter detectors. These jets are paramount features of modern high energy proton-proton colliders such as the Tevatron and LHC.

We can draw a general picture of compositeness (Figure 1.4). At low energies, the internal structure of composite objects seems to be point-like and elementary. Then, after some energy threshold, deviations in the expected elementary couplings can be observed, and kinematic effects appear through form factors of interactions. Eventually, experiments get energetic enough to produce the composite states and all the composite Hadron-like objects emerge. Finally, the UV theory is manifested when energies are much above the confinement scale of the theory.

Compositeness is a scale-dependent concept, and it reveals new physics benchmarks. These new benchmarks generally come with new interactions and new particles as unique experimental features. Given that, why would the Higgs be a composite particle?

The answer comes with the role of the Higgs boson in EWSB. A composite 


\begin{tabular}{|l|ccccc|}
\hline & $q_{L}$ & $u_{R}^{c}$ & $d_{R}^{c}$ & $l_{L}$ & $e_{R}^{c}$ \\
\hline$S U(3)_{c}$ & 3 & 3 & 3 & 1 & 1 \\
$S U(2)_{L}$ & 2 & 1 & 1 & 2 & 1 \\
$U(1)_{Y}$ & $1 / 6$ & $-2 / 3$ & $1 / 3$ & $-1 / 2$ & 1 \\
\hline
\end{tabular}

TABLE 1.2: Quantum numbers of the SM elementary fermions. There are three quark fields $Q, U^{c}, D^{c}$ and two lepton ones $L$ and $E^{c}$.

Higgs would signal the existence of yet another benchmark roughly at the $\mathrm{TeV}$ range. Ultimately, we expect that the presence of a new energy benchmark would complete the SM Higgs mechanism, promoting it to a dynamical EWSB.

As it is, the SM is the elementary theory of strong, weak and electromagnetic interactions. It provides the unification of Electroweak (EW) phenomena through the Weinberg-Glashow-Salam theory of local $S U(2)_{L} \times U(1)_{Y}$ interactions $[4,2,3]$.

The gauge structure of the Electroweak sector is very well established. It is built over a local group $S U(2)_{L} \times U(1)_{Y}$, so all mass terms are forbidden by the chiral $S U(2)_{L}$ symmetry and gauge invariance. Therefore, without the Higgs mechanism for SSB, all particles should be massless.

Before SSB, there are four massless gauge bosons. Three of them correspond to the generators of $S U(2)_{L}$ and one with $U(1)_{Y}$. So, these are $W_{1}^{\mu}, W_{2}^{\mu}, W_{3}^{\mu}$ and $B^{\mu}$.

Additionally, the SM includes 12 elementary fermions, divided between three generations of quarks and leptons. These are organized in a singlet/doublet structure, in which the right-handed fields are singlets, and left-handed fields are doublets of $S U(2)_{L}$. Each one of these has a Hypercharge and $S U(3)_{c}$ charge as specified in table 1.2. Furthermore, these doublets are composed between two fermions of the same generation (i.e. up/down, electron/neutrino $\left(\nu_{e}\right)$, etc).

Altogether, the Electroweak part of the SM is

$$
\begin{aligned}
\mathcal{L}= & -\frac{1}{4} W_{a}^{\mu \nu} W_{\mu \nu}^{a}-\frac{1}{4} B^{\mu \nu} B_{\mu \nu}+i \overline{f_{L, i}} \not D f_{L, i}+i \overline{f_{R, i}} \not D f_{R, i} \\
& +\underbrace{\left|D_{\mu} H\right|^{2}+V\left(|H|^{2}\right)+y_{i j} \overline{f_{L, i}} H f_{R, j}+h . c .}_{\text {Higgs Sector }}
\end{aligned}
$$


Unification of Electroweak phenomena requires the embedding of an $U(1)_{E M}$ into the SM group. This is achieved by the SSB of $S U(2)_{L} \times U(1)_{Y} \rightarrow U(1)_{E M}$, which triggers the Higgs mechanism.

The Higgs field is defined as a scalar that interacts with the Electroweak gauge bosons and charged fermions. Its quantum numbers must be fixed by physical requirements of EWSB.

Meaningfully, the SSB process indicates that the vacuum state is not symmetric under the full EW group. In this phase, the Higgs assumes a vacuum expectation value (vev), $\langle H\rangle=v$. EWSB triggers the Higgs mechanism because the interactions of the Higgs generate mass terms when $H$ it assumes its vev. The fact that $U(1)_{E M}$ is left unbroken indicates the masslessness of the photon.

So, out of these four gauge bosons, three of them get masses due to the Higgs [14, 15]. Also, because their mass terms are components of the Higgs field itself, we need at least three degrees of freedom into the $H$ multiplet. The smallest scalar that contain such three components is $H \sim\left(\mathbf{1}, \mathbf{2},+\frac{1}{2}\right)^{3}$.

$$
H=\frac{1}{\sqrt{2}}\left(\begin{array}{l}
h_{1}+i h_{2} \\
h_{3}+i h_{4}
\end{array}\right)
$$

Keeping only renormalizable terms in the Higgs potential, the Higgs sector lagrangian is ${ }^{4}$ :

$$
\begin{aligned}
\mathcal{L}_{H} & =\left|D_{\mu} H\right|^{2}+\mu^{2} H^{\dagger} H-\lambda\left(H^{\dagger} H\right)^{2}+ \\
& -\overline{l_{L}} y_{e} H e_{R}-\overline{q_{L}} y_{d} H d_{R}-\overline{q_{L}} y_{u} \tilde{H} u_{R}
\end{aligned}
$$

where $y_{i}$ are the Yukawa matrices in flavour space. The covariant derivative is:

$$
D_{\mu} H=\left(\partial_{\mu}-i g W_{\mu}^{i} t^{i}-i g^{\prime} Y_{H} B_{\mu}\right) H
$$

\footnotetext{
${ }^{3}$ The notation here means $\left(S U(3)_{c}, S U(2)_{L}, U(1)_{Y}\right)$ charges. Additionally, the Higgs hypercharge is taken as an input of the theory and fixes all other SM hypercharges.

${ }^{4}$ In which $\tilde{H} \equiv i \sigma_{2} H$
} 
In order to guarantee the positivity of the potential, $\lambda>0$. Also, there are two phases, defined by the sign of $\mu^{2}$. If $\mu^{2}<0$ the potential has a trivial minimum at $\langle H\rangle=0$, thus with no EWSB. If the potential has $\mu^{2}>0$, we can trigger EWSB since there will be a nontrivial minima. Now, minimizing such potential lead to the definition of the Higgs vev as $|\langle H\rangle|^{2}=\frac{\mu^{2}}{\lambda}$.

One can see how the mass terms arrise from the Higgs sector Lagrangian. First the gauge boson massses come from the cotribution of the "veved" Higgs in its kinetic term.

$$
\begin{aligned}
\left|D_{\mu}\langle H\rangle\right|^{2}=\frac{1}{4} & {\left[g^{2}\left(W_{\mu}^{1}-i W_{\mu}^{2}\right)\left(W_{1}^{\mu}+i W_{2}^{\mu}\right) v^{2}\right.} \\
& \left.\left.+\left(-g W_{\mu}^{3}+g^{\prime} B_{\mu}\right)^{2} v^{2}\right)\right]
\end{aligned}
$$

We define the mass basis for the gauge bosons as $W_{\mu}^{ \pm}=W_{\mu}^{1} \mp i W_{\mu}^{2}$ and $Z^{\mu}=$ $\frac{1}{\sqrt{g+g^{\prime}}}\left(g W_{\mu}^{3}-g^{\prime} B_{\mu}\right)$. Substituting the basis back in (1.9) leads to

$$
\begin{aligned}
& \left|D_{\mu}\langle H\rangle\right|^{2}=m_{W}^{2} W_{\mu}^{+} W^{-, \mu}+\frac{m_{Z}^{2}}{2} Z_{\mu} Z^{\mu} \\
& m_{W}^{2}=\frac{g^{2} v^{2}}{2} \\
& m_{Z}^{2}=\frac{\left(g^{2}+g^{\prime 2}\right) v^{2}}{2}
\end{aligned}
$$

Defined by $A^{\mu}=\frac{1}{\sqrt{g+g^{\prime}}}\left(g W_{\mu}^{3}+g^{\prime} B_{\mu}\right)$, the photon is left massless in the broken phase of the SM.

The fermionic mass terms are generated by the Yukawa lagrangian.

$$
\mathcal{L}_{Y u k}(\langle H\rangle)=-\frac{v}{\sqrt{2}} \overline{e_{L}} y_{e} e_{R}-\frac{v}{\sqrt{2}} \overline{d_{L}} y_{d} d_{R}-\frac{v}{\sqrt{2}} \overline{u_{L}} y_{u} u_{R}
$$

Going to the mass basis in flavour space defines the mass-Yukawa relation, a consequence of the SM Higgs mechanism.

$$
m_{f}=\frac{y_{f}}{\sqrt{2}} v
$$




\begin{tabular}{|l|l|}
\hline Measured quantity & Experimental value \\
\hline Anomalous $g_{e}-2$ & $0.0011 \cdots \pm 2.8 \times 10^{-13}$ \\
$G_{F}$ (Muon Lifetime) & $1.1663787(6) \times 10^{-5} \mathrm{GeV}^{-2}$ \\
Z pole mass (LEP) & $91.1876(21) \mathrm{GeV}$ \\
Z decay width (LEP) & $2.4952(23) \mathrm{GeV}$ \\
Z polarizarion assymetry & $0.1515(19)$ \\
\hline
\end{tabular}

TABLE 1.3: Experimental values SM observables.

Fixing the vev, the SM requires that $g, g^{\prime}$ and all the Yukawa couplings must be known to reproduce the particle spectrum. In total, there are two parameters for the gauge sector, 2 for the Higgs, and 13 for the whole Yukawa sector ${ }^{5}$.

Even with a lot of independent parameters, the SM predictions are very concrete. When confronted with the experiment, the SM has proven to have a remarkably robust structure. Since the 1970's, its internal structure is intact, without any modification dictated by experimental data.

The success of the SM gauge sector is reflected in the astounding precision of the electron magnetic moment and muon lifetime measurements. Furthermore, at the Large Electron-Positron (LEP) collider, Electroweak Precision Tests (EWPT) were conducted to study loop-level properties of the massive electroweak gauge bosons $[6,13]$. The results indicate the experimental sturdiness of the gauge interactions that all agree with the SM predictions, and are indicated in table 1.3.

Subsequently to the LEP experiments, the LHC presented the possibility of finding the Higgs. The objective was the discovery, the study of its couplings as well as its production/decay channels.

The main production channels of the Higgs are gluon fusion, Higgs-strahlung, vector fusion and top fusion. Furthermore, the decays of the Higgs are mostly to the bottom-quark, the most massive kinematically available fermion. Even though it is the dominant mode, there are also several opportunities to study other kinds of decay channels, as it can be seen in figure 1.5 below.

\footnotetext{
${ }^{5}$ These are three Yukawa couplings for each lepton, up- and down quarks, plus three CKM angles and a CKM phase.
} 

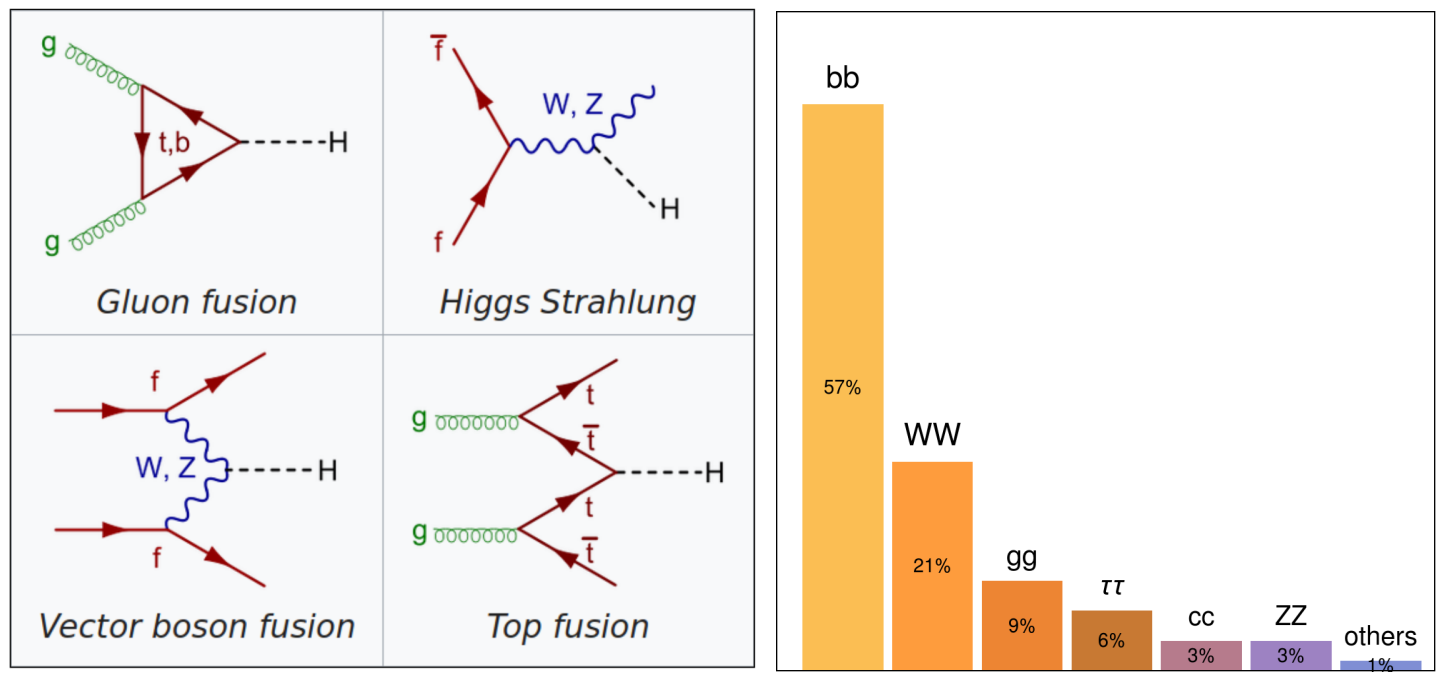

FiguRE 1.5: Main production and decay channels of the Higgs. The diagrams of the most important production channels are to the left, and the branching ratios of Higgs decays to the right.

In 2012, the Higgs was discovered, confirming the SM predictions [7, 8]. The Higgs discovery is indicated in figure 1.6. It was a combined analysis of multiple channels dominated by the decay of the Higgs to two photons. Even though this decay mode has a small branching fraction, $B R(H \rightarrow \gamma \gamma)=1.73 \%$, it is preferred since the detector signal is cleaner. This illustrates that the production cross-section and branching ratio analysis are not the only factors when determining the Higgs visibility.

A fair share of the Higgs couplings was observed in the LHC. In all measurements, the couplings are in agreement with the SM predictions, and there are no favoured results for BSM searches, as pictured in figure 1.7.

In short, the experimental picture of the SM is very favourable. We are already probing scales much beyond the Electroweak, at $13 \mathrm{TeV}$ and the SM predictions are still compatible. However, there is much concern regarding the theoretical framework we traced.

The most critical issue is regarding the inclusion of BSM effects. Certainly, the SM is not the final picture of nature - there are still many observed phenomena that require modification. We have an undoubtful amount of evidence for dark matter; 

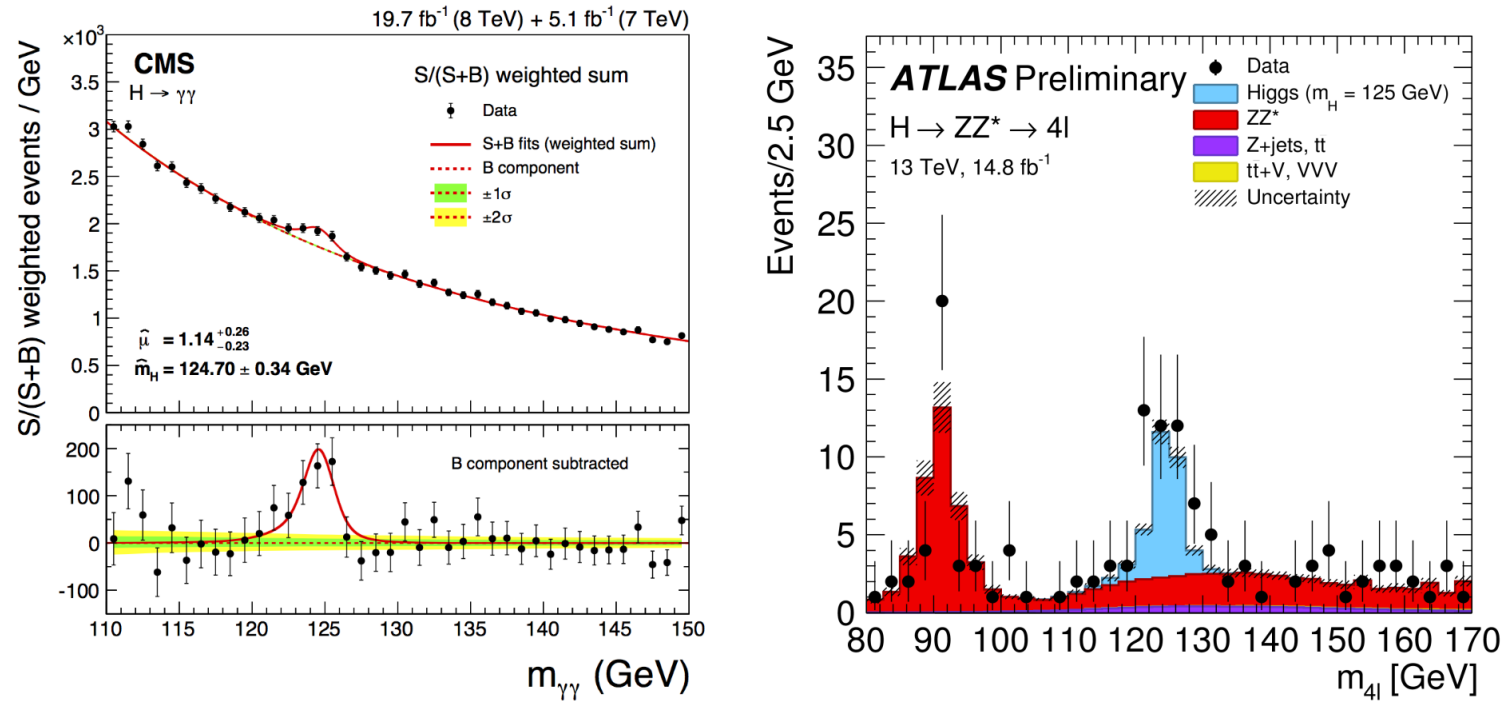

FiguRE 1.6: Higgs discovery plots. The sharp peak indicates the presence of the Higgs boson at $m_{h}=125 \mathrm{GeV}$. To the left, is the number of events of $H \rightarrow \gamma \gamma$ as a function of the invariant mass distribution $m_{\gamma \gamma}$. S and B are the numbers of signal and background events, respectively. To the right, is the $H \rightarrow 4 l$ number of events per $m_{4 l}$. In both the discovery significance of $5 \sigma$ was reached by an analysis of multiple production channels.

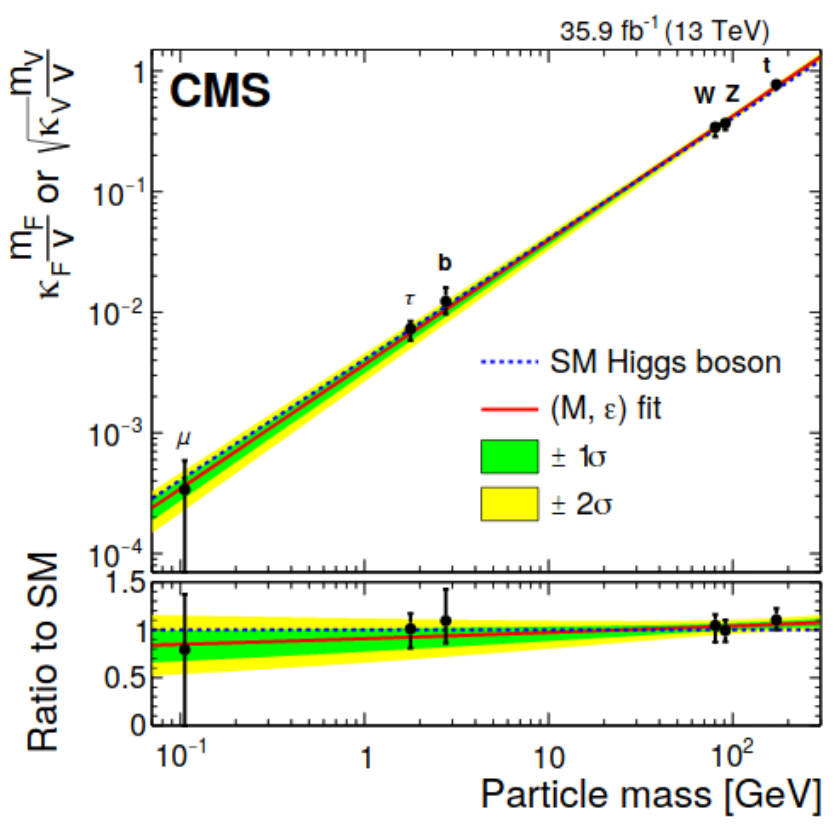

FiguRE 1.7: Measurements of the Higgs couplings at the LHC. Data from run 2. So far, the Higgs couplings to the top/bottom quarks, tau/muon leptons and $\mathrm{W} / \mathrm{Z}$ gauge bosons were measured. The dashed line is the SM expectation. Green and Yellow contours are the $1 \sigma$ and $2 \sigma$ C.L. intervals. The measurements are in agreement with predictions as indicated by the red line fit. 
we have not identified the scale responsible for the neutrino masses; another energy benchmark could be responsible to resolving the flavour puzzle of fermion masses; the running of gauge couplings suggest complete unification at high energies; there is matter-antimatter asymmetry; and, ultimately, gravity forces a completely new picture at the Planck scale. All of these are not included in the SM framework [47].

Here lies the experimental tension between theory and experiment - The Higgs, as a scalar particle, lives at the cutoff for new physics. As an elementary particle, it has not enough symmetry to protect its mass from divergent contributions due to its interactions. Since the Higgs should live at the cutoff, whenever we introduce new physics at higher scales, the Higgs mass value is driven towards this scale.

In such a conflict, we define the hierarchy problem. The elementary SM presents a remarkable resilience to BSM inclusions, yet, we need to incorporate BSM phenomena.

A simple calculation illustrates this effect. The most important loop effects to the Higgs mass comes from virtual top quark corrections, massive gauge boson, and self-interaction loops. All of these yield quadratically divergent contributions.

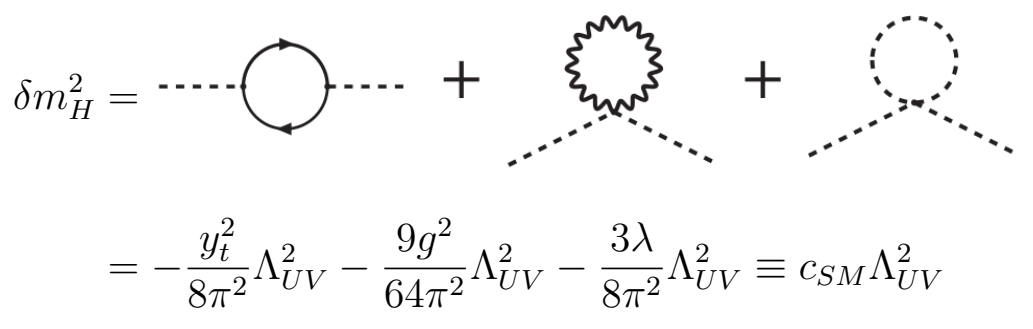

The hierarchy problem appears when we have to fix the physical Higgs mass to its $125 \mathrm{GeV}$ value. To fix it, we subtract the divergences from a counterterm that parametrizes the ultraviolet (UV) unknown physics.

$$
\begin{aligned}
\overbrace{m_{H}^{2}}^{\text {physical mass }} & =m_{\mathrm{BSM}}{ }^{2}-\left|\Delta m_{H}^{2}\right| \\
& =\left(c_{B S M}-c_{S M}\right) \Lambda_{U V}^{2}
\end{aligned}
$$


As we try to send the cutoff of the SM to $\Lambda_{U V}^{2}>>m_{H}^{2}=(125 \mathrm{GeV})^{2}$, we face the problem of fine-tuning the combination $\left(c_{B S M}-c_{S M}\right)$ to be very small. This makes the inclusion of heavy phenomena very unnatural.

The Hierarchy problem is not about cancelling divergences, it is about the separation of the Electroweak and BSM scales. As heavier as the new physics scale is, the stronger we have to fine-tune. For this reason, we expected new physics at the $T e V$ s. If it comes from higher energies, the model becomes uncontrollably unnatural. Yet, no new $T e V$ physics showed up so far.

Due to this puzzling outcome, the theoretical pathway is gradually being changed. The sole confirmation of the SM, without new phenomena, confronts particle physicists expectation and forces us to rethink our expectations of BSM physics. The SM picture with an elementary Higgs is the most striking puzzle we face today.

\subsection{The Compositeness of the Higgs Boson}

In nature, SSB is a reasonably common phenomenon ranging from ferromagnetism, superconductivity and the melting of ice to the Hadronic structure given by QCD. In all of these, there are broken and unbroken phases that define the physical systems, each with its unique properties. These spontaneously broken systems can be described by their order parameters and effective theories (i.e. Landau-Ginsburg Theory; chiral perturbation theory). However, it often happens that the SSB process itself has a microscopic dynamical origin. For example, superconductivity can be described by the effective Landau-Ginsburg theory, but its microscopic origin is only provided by the condensation of cooper pairs as in BCS theory.

In the case of the SM, the HP seems to suggest more structure to EWSB than what is contained in the Higgs Mechanism. An elementary Higgs signals a structureless agent of EWSB, which indicate the absence of dynamics to EWSB. As it is, the Higgs mechanism can describe the properties of EWSB in the broken phase but gives no reason on how the EW scale comes to be stable. 


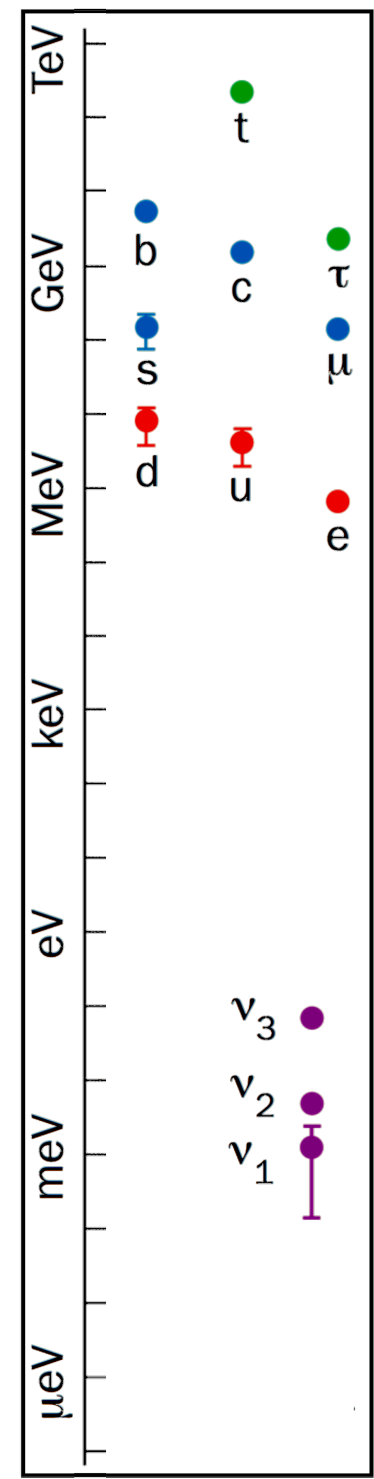

Figure 1.8: The flavour puzzle. Here, the Higgs is responsible for generating all mass terms (perhaps, apart from the $\nu$ 's). Hierarchy is present in fermion mass parameters, ranging across six orders of magnitude. Together with the HP, the fermion hierarchy are puzzles of the Higgs sector formulation.
The Higgs mechanism just parametrizes EWSB. It provides no insight into a possible microscopic origin of such phenomena. Simply, the elementary Higgs is assumed to be in its broken phase $\left(\mu^{2}>0\right)$, but there are no dynamics that drove it to such a picture. We are forced to assume the EW vev as an input of the theory, defining the only scale of the SM. This assumption is not a problem within the SM itself, but with a new energy benchmark, we are bound to introduce hierarchy. If EWSB is not dynamical, $v$ cannot be generated from the next scale, and fine-tuning problems start to arise.

Similarly, another hierarchy of scales regards the origin of fermion masses. As we have seen, the gauge sector has an intrinsic and well-defined structure with great experimental confirmation. However, the fermionic sector comes with plenty of independent parameters. Their values range across six orders of magnitude, which fixes the fermion masses at their physical values, as indicated in figure 1.8.

This might suggest that there is a scale, yet unidentified, that sets up the hierarchy within the fermion families. Both the HP and the hierarchy of fermion masses are related to the Higgs sector of the SM.

A composite solution to the HP that also addresses the origin of fermion masses origin was first presented by Georgi and Kaplan [20, 21, 22]. Their core ideas were further improved in the context of 5-dimensional theories by Pomarol, Agashe, Contino and many others [23, 24, 26, 28]. The $5 \mathrm{~d}$ models match the $4 \mathrm{~d}$ effective 
description we will be using throughout this work. This framework is generically called Composite Higgs Models (CHMs).

In such class of models, the Higgs is assumed to be part of a composite sector that confines its fundamental constituents at the $T e V$ scale. Inspired by the pions in QCD, the Higgs of CHMs is made naturally light by being a pseudo-NambuGoldstone (pNGB), exploring a consequence of the Goldstone Theorem. Ultimately, this feature explains why the Higgs is not at the SM cutoff, and why it can be fixed at the EW scale. Therefore, this solution to the HP requires both compositeness and the use of symmetry to achieve naturalness.

CHMs provide a way out of the HP by dismantling the Higgs at high energies, in analogy with the case of hadrons mentioned earlier. These models provide a robust framework to compute interaction shifts from the SM, and they allow estimates of how light its mass can naturally be. Ultimately, they are effective local descriptions of an unknown UV completion. Deep into the UV, the actual constituents of the Higgs should be revealed just as the constituents of hadrons did.

Besides these features, CHMs lack the main ingredient of compositeness - momentum dependence.

The goal here is to study in such models the signature of compositeness and to explore their phenomenology.

As we shall review, the predicted kinematic distribution functions of CHMs are not altered in the effective description of the theory, and the general picture of form factors is not fully incorporated to parametrize compositeness effects to their full extent. The general problem is, how does one look for general signals of the compositeness of the Higgs boson at collider experiments?

To this end, this work is devoted to finding a systematic way of including the Higgs compositeness effects and finding the means to study its phenomenology. 
This dissertation is organized as follows. Chapter 2 is dedicated to reviewing the CHM framework in search of compositeness effects. These momentum dependent effects will be parametrized by form factors in a similar manner as in hadronic interactions. However, as defined in the literature, the formulation is incomplete since the Higgs is non-dynamical. This restricts the full dependence on the momentum of Higgs interactions and hides away the phenomenological signals.

The objective will be to restore the complete momentum dependence of the Higgs interactions in form factor models.

The construction of the form factor theory will be addressed in chapter 3 . There, we will introduce a set of nonperturbative methods for dealing with strong interactions. We shall build the form factors from general requirements like the spectral function, and use tools to write down explicit expressions for them.

We achieve a concrete implementation of momentum dependence in chapter 4 , through a minimal realization of the composite Higgs. Subsequently, we study the collider signatures of compositeness through form factor models in chapter 5 .

In chapter 6, we conclude with a discussion on the picture of the Higgs compositeness. The focus is to present distinct possibilities that might overcome the usual paradigms of model-building compositeness effects. Finally, we present some prospects for future research and the final comments. 



\section{Chapter 2}

\section{A Composite Higgs Boson}

After the developments of the Standard model, there were several attempts to realize the Higgs as a composite state providing a dynamical origin to EWSB. Much of the difficulties arise in the aversion of the SM to BSM physics, which generally require the alternatives to be very SM-like.

There are some presumptions to the composite Higgs hypothesis. Necessarily, the Higgs should be accompanied by a whole new sector that modifies the SM predictions, while also being experimentally consistent with the collider searches so far. CHMs offers such consistency and will serve as a concrete framework to build the phenomenology later on.

As a first goal, we aim to find the ingredients of models with composite Higgses and learn to compute the modifications with respect to the SM. Then, we seek to identify what are the primary effects of compositeness on physical observables, going beyond the local description of these models.

This chapter is devoted to reviewing the models in which the Higgs is a composite bound state. We shall look for a complete description, in search of the distinctive features of composite phenomenology.

As we have seen, compositeness is a scale-dependent concept. So by making the Higgs composite, we are assuming the existence of another type of interaction, yet unobserved, that is responsible for bound it at the $\mathrm{TeV}$ range. The fundamental theory must be such that the constituents are not clearly produced in collider experiments. They must be much above the TeV's into the UV. Because of this, 
the general expectancy is that the new sector interacts strongly to confine its fundamental constituents, much like what happens in QCD.

Because of the Higgs narrowness, we do not see its emergence as a loosely bounded composite. To ensure compatibility with experimental results, the underlying sector must be strongly coupled.

Therefore, to realize the composite Higgs, it is necessary to know some general properties of strongly interacting theories. This is a hard task since the formation of bound states depends on nonperturbative physics, and there are limited tools to treat such problems. Model building becomes a tough task for such strong interactions. In chapter 3, we shall explore some available nonperturbative tools to explore these sectors with more depth.

Fortunately, discovering the fundamental theory that underlies the composite states is not the crucial step from the low energy point of view. It is appropriate to use the language of effective field theories to treat the IR theory. In such effective parametrization, we assume some underlying symmetry structure to be respected, through which we build a Lagrangian model.

The primal example of a nonperturbative theory is QCD. Because of its importance, almost all tools we have for analyzing the strongly coupled theories are QCD biased, and much of the phenomenology of CHM bears some resemblance to Hadronic models.

The QCD experience suggested that in a confining theory, we expect bound states as resonances. If the confinement scale of the new composite sector is $f$, we define the spectrum by the masses $m_{*}=g_{*} f$ in which $g_{*}$ are the coupling constants of the theory. In general, these particles are close to the confinement scale and tightly split from each other.

Namely, all the attempts to make the Higgs composite rely on this structure we have a spectrum of resonances in which the Higgs emerges amongst them. Such is portrayed in figure 2.1 .

Now, the input scale is the dynamically generated $f$, an order parameter for 


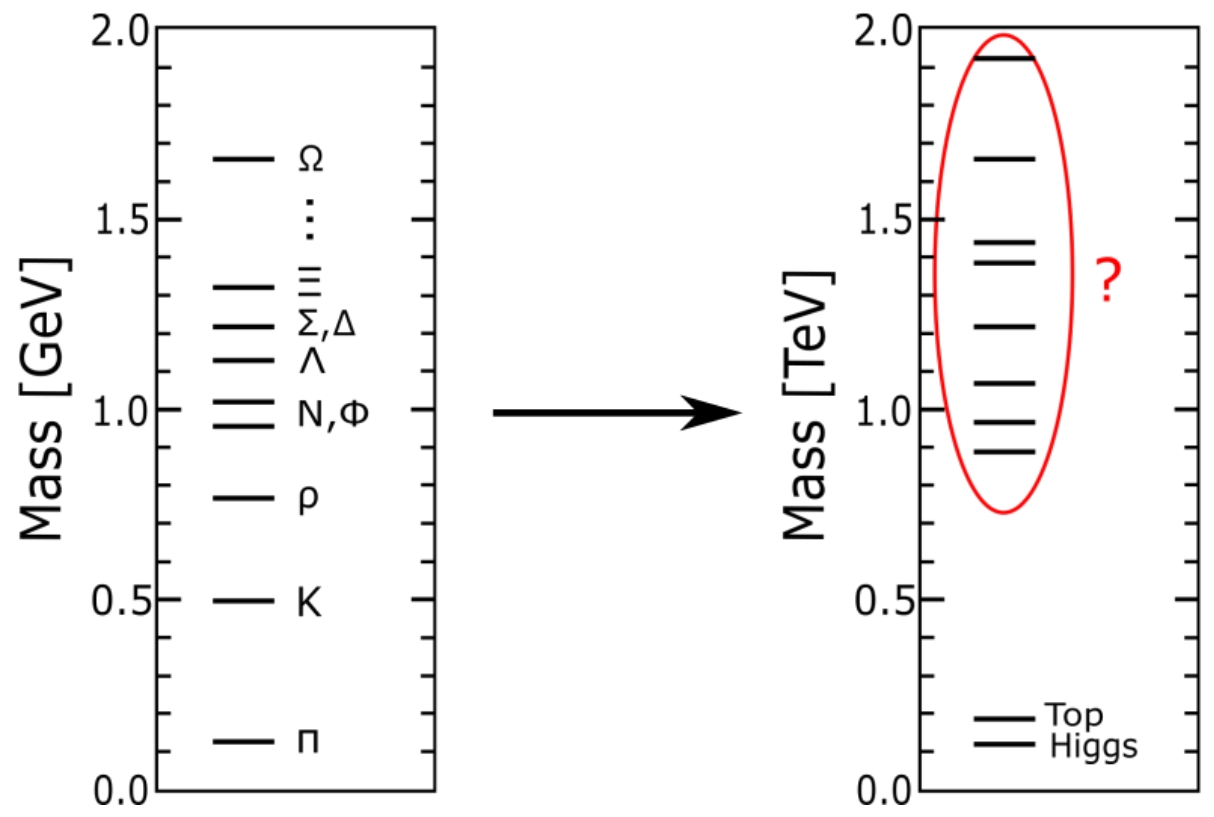

FIGURE 2.1: Hadron spectrum parallel with the composite sector spectrum. The analogy between CHM and QCD is extensive. We have pNGBs in both theories - the pions in QCD and the Higgs in CHMs. Also, we assume a spectrum of resonances for the new sector that resembles the hadronic structure.

the confinement process. The EW scale must be generated from this $f$ to address the hierarchy issue. In this way, we are ensuring a new dynamical content to EWSB that should complete the Higgs role in the SM.

As stressed, the main challenge in constructing a feasible composite Higgs is due to the empirical adequacy of the SM. Because of this, the form of the Higgs must be very similar to what the SM predicts. Here lies the main challenge to exotic BSM physics; the internal structure of the SM seems to be very BSM-phobic in this matter.

As the experimental data continues to agree with the SM, the attitude of the community is - how well a model survives the SM limit gives how successful it will be. This underlies the success of a class of models which make the Higgs a pNGB composite. In these models, we reobtain the SM by sending the new physics scale $f$ to infinity, at the expense of reintroducing tuning in theory.

This has been the central theme of theoretical research in the past years. As 
data get more aligned with the SM, model-building of BSM effects is more tweaked to reproduce SM predictions.

Conversely, the first attempt to give a dynamical origin to EWSB, was a complete departure of the SM mechanism. This was Technicolor [45]. Like in QCD, Technicolor is assumed to be a confining theory and has a confinement energy scale. The Higgs emerges as a loosely bound state, similarly to cooper pairs in superconductor theories. Here, the insight from superconductivity to the Higgs mechanism becomes even more explicit, where it is assumed that a pair of techniquarks combine to give rise to the Higgs. Unfortunately, Technicolor seems not to have lived up to its full potential.

The discovery of a narrow light Higgs mostly discard Technicolor models. The main difficulty is that the Higgs is like the $\sigma$ meson in QCD; it lives close to $f$. Being close to the compositeness scale, it becomes naturally heavy and much broader than the SM predictions.

A solution for these phenomenological conundrums is to make the Higgs analogous to pions in QCD. The pions are naturally light compared to the QCD scale since they are pseudo-Nambu-Goldstone bosons of the broken chiral symmetry. Inspired by this, protecting the Higgs mass by the Goldstone mechanism turned out to be the primary motivation of CHMs.

The Composite pNGB Higgs provides a particular and concrete implementation of the Compositeness idea for the Higgs. The focus of this chapter is to perform an inquiring review of pNGB Composite Higgs Models, craving for the phenomenological signatures of Higgs compositeness.

So, in which ways the pNGB Composite Higgs are successful models and where are the compositeness signs in the phenomenology?

We will explore the construction of the global symmetry breaking pattern. Such construction underlies the role of the new strongly interacting sector in which 
the Higgs emerges at the TeV scale. The EWSB dynamical origin will be reviewed via the Vacuum Misalignment mechanism, which is one of the main conceptual pillars of composite Higgs models.

Then, the parametrization of the theory can be done by realizing symmetries non-linearly. This approach provides a systematic language for describing the low energy degrees of freedom of a theory that has NGB's. Finally, we will be able to extract the Composite Higgs interactions and compute its modifications for the Higgs couplings to fermions and gauge bosons.

To get a light Higgs and to solve the Hierarchy problem we will sketch the calculation of the Coleman-Weinberg potential. This computation will require a formalism that shall put us in the track for the main subject of this work - the Form Factor parametrization.

\subsection{The pseudo-Nambu-Goldstone Higgs Particle}

To work out the meaning of CHMs, let us distillate of the composite pseudoNambu-Goldstone Higgs particle. First, the "Nambu-Goldstone" contribution refers to the Goldstone Theorem.

\section{Goldstone Theorem}

In the broken phase, "whenever the original Lagrangian has a continuous symmetry group, the new solutions have a reduced symmetry and contain massless bosons." a

\footnotetext{
${ }^{a}$ Taken from Goldstone's paper [5].
}

These massless states are called the Nambu-Goldstone Bosons, and they are very general in any quantum theory. A continuous global symmetry implies the existence of a Noether current $J(x)$ which is conserved ${ }^{1} \partial_{\mu} J(x)=0$. Then, if the symmetric vacuum with the conserved charge $Q|0\rangle_{\text {Sym }}=0$ is unstable, and the true vacuum is charged $Q|0\rangle \neq 0$, we are by definition pledged to symmetry breaking.

\footnotetext{
${ }^{1}$ We are assuming this conservation remains exact at the quantum level.
} 
Physically, what is happening is that the global symmetry forces the potential to be either unique, with no symmetry breaking, or degenerate, then with symmetry breaking. The running modes on the degenerate vacuum are the Goldstone bosons like shown in figure 2.2 .
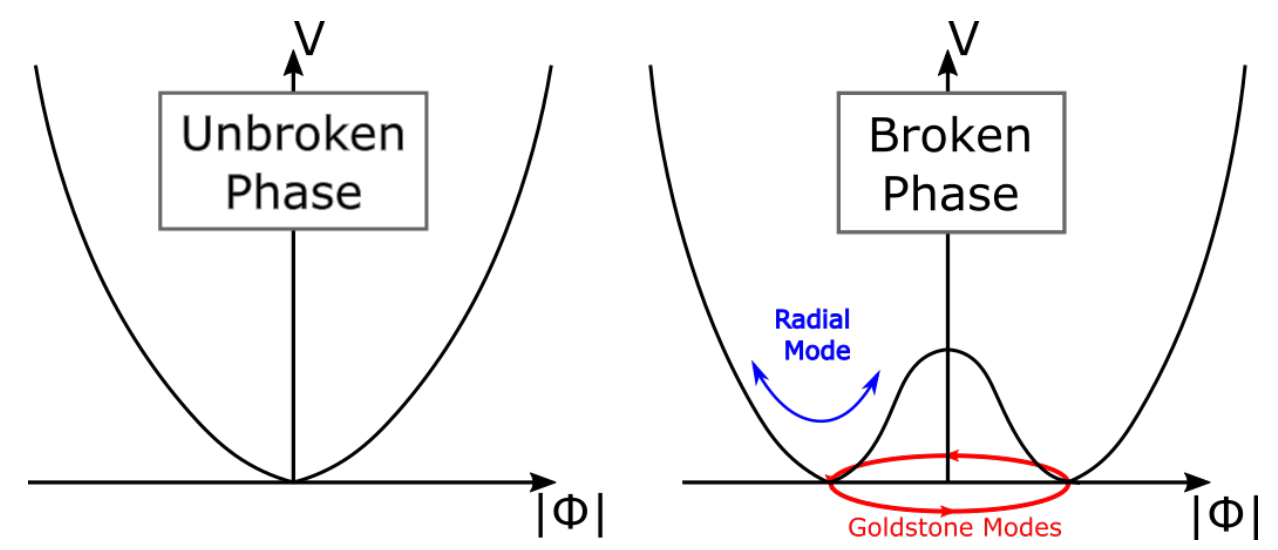

FiguRE 2.2: SSB of a potential V. There are two phases in a system that can have SSB - the unbroken one is defined by the trivial vacuum state, with no expectation value. The broken phase has a degenerated vacuum with associated massless Goldstone bosons.

When the starting symmetry is exact, the goldstone bosons are massless. However, if the continuous global symmetry is softly broken, the Nambu-Goldstones become pseudo-Nambu-Goldstones acquiring a small mass value. This process is pictured in figure 2.3 .

The smallness of the Goldstone masses is referring to the mass gap created between the typical scale of the theory and the much lighter, SSB protected scale. We already see why this mechanism is of interest here, it creates hierarchy in a natural way ${ }^{2}$.

In light of the Goldstone theorem, a scalar boson can be lighter than the associated physical scale of the theory. Remember that the Hierarchy problem is just about that: How can the Higgs be at the electroweak scale when we expect it to be far above that?

\footnotetext{
${ }^{2}$ As natural as the source of explicit breaking can be.
} 

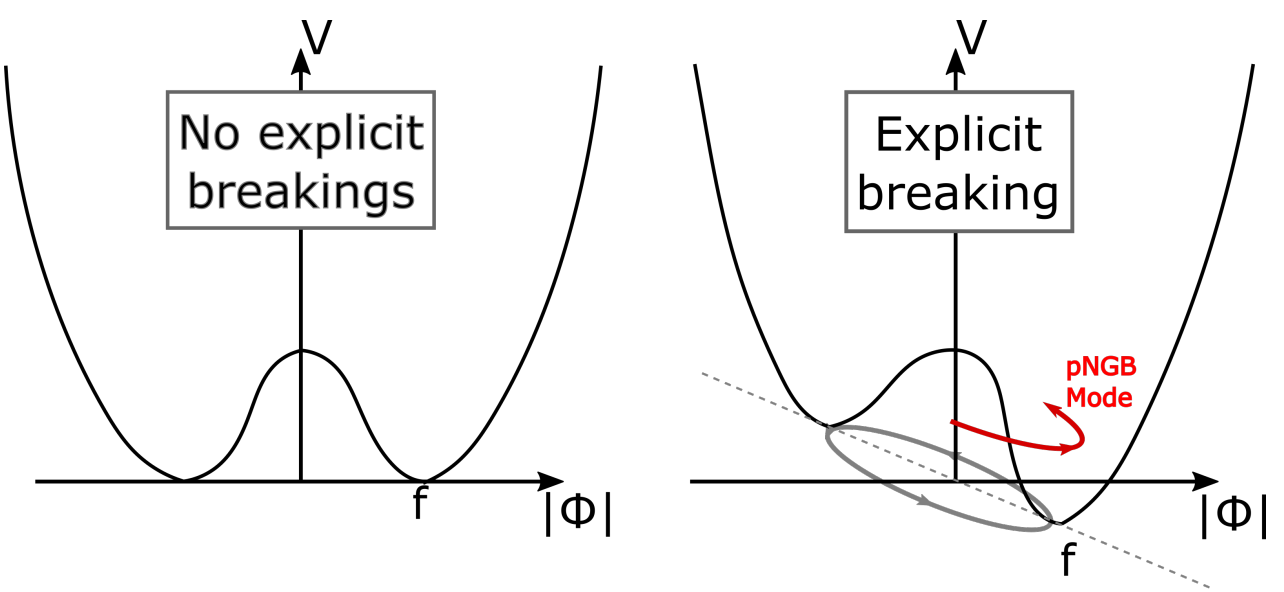

FIGURE 2.3: SSB in the broken phase, with or without explicit breaking. If there is no explicit breaking, the Goldstone bosons are massless as predicted. However, when introducing explicit breakings, they can acquire masses. If the breaking is soft, then we expect these states to be lighter than the rest of the theory spectrum.

One could jump to the punchline directly - The Higgs shall be a pseudoNambu-Goldstone boson.

The Goldstone theorem refers to the spontaneous symmetry breakdown of global continuous symmetries and in principle does not apply to the Higgs mechanism, which explores the structure of the local Electroweak gauge group $S U(2)_{L} \times$ $U(1)_{Y}$. The apparent loophole of the Goldstone's theorem for EWSB is explained by the longe range correlations imposed by the gauge bosons of the theory. These long-range correlations wash out the goldstone degrees of freedom, which becomes the longitudinal component of the gauge bosons.

Importantly, we must distinguish between the two symmetry breaking events occurring. One is the familiar Electroweak symmetry breaking that happens when the Higgs assumes its vacuum expectation value. Concerning this breaking, the Higgs particle is the radial mode and not a Goldstone. Because of the local structure, the "would-be" goldstone from the other Higgs doublet components are eaten up by the W's and Z particles, which become massive as a result. No Goldstone's here, just massive vectors.

The other symmetry breaking is due to a new global symmetry that is not 
manifest in the Standard Model formulation. Here lies the CHMs proposal. We will call the symmetry group $\mathcal{G}$ which breaks to a subgroup $\mathcal{H}$, and the Higgs end as a Nambu-Goldstone of this breaking, precisely as the Goldstone theorem predicts.

Even though the two breaking patterns seem to be independent of each other, we require a more intrinsic connection between them. Ultimately, we want that the presence of the strongly coupled sector should trigger a dynamical EWSB.

To achieve the dynamical EWSB, the generation of the Higgs potential shall be induced by explicit breakings of $\mathcal{G}$. These breakings are introduced by the interactions between the elementary particles and members of the composite sector. This arrangement is accomplished through what is called the vacuum misalignment mechanism and is at the core of CHMs.

The composite pseudo-Nambu-Goldstone Higgs particle gives a starting point on what will be needed to construct viable composite Higgses, and complete the picture of compositeness.

\subsubsection{A New Composite Sector}

We shall turn to describe the theory at the confinement scale effectively. At this level, there are several new composite heavy states and the remaining elementary fields of the SM. The objective is to describe the composite and elementary sectors, together with the interactions between the two of them.

$$
\mathcal{L}=\mathcal{L}_{C S}+\mathcal{L}_{E S}+\mathcal{L}_{\text {int }}
$$

As we have seen, to have a Goldstone Higgs we need to extend the SM group to incorporate a larger global symmetry. Then this global symmetry should be broken, and the Higgs field should transform with the coset generators since it lives in $\mathcal{G} / \mathcal{H}$.

$$
\mathcal{G} \rightarrow \mathcal{H}
$$




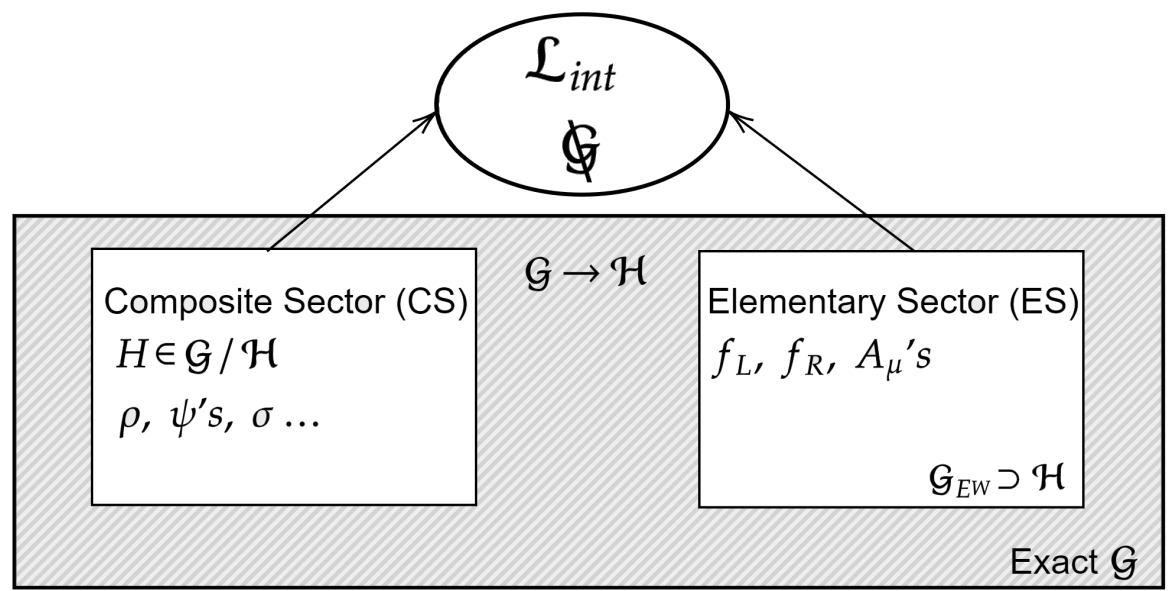

Figure 2.4: Scheme of a pNGB CHM. The global $\mathcal{G}$ is used to build the theory. Individually, the elementary and composite sectors have an exact $\mathcal{G}$ symmetry. The $\mathrm{SM}$ is embedded into $\mathcal{H}$ the unbroken subgroup of the global SSB. Interactions between the two sectors are introduced, explicitly braking $\mathcal{G}$, and making the Higgs a pNGB.

The theory can be divided into a pure composite sector, a pure Electroweak without the Higgs and the interactions between the two. This scheme is portrayed in figure 2.4.

The composite sector arises from the condensation of the UV degrees of freedom in the more fundamental theory. Similarly to QCD, we assume that this process gives rise to composite states much like the Hadrons, which we generically name as resonances. Additionally, the Higgs should be part of this spectrum, since it arises as a composite. Concerning this sector alone, the $\mathcal{G}$ symmetry is exact, and the Higgs is a true Goldstone boson.

Then, there is the pure Elementary sector. Here is located all remaining SM fields apart from the composite Higgs. In this sector all fermions and gauge bosons are massless, and even though chiral symmetry was exact in the SM, now it is utterly manifest with no mass terms.

Finally, EWSB is achieved through interactions between the Composite and Elementary sectors. A mass gap is generated, and the Higgs is maintained at electroweak scales naturally.

Practically, this structure is realized by embedding the standard model in 
the broken subgroup $\mathcal{H}$ and then generating EWSB via interactions between the elementary and composite sectors. Such is the general procedure proposed by Georgi and Kaplan [20, 21, 22].

The mass gap indicates that it would be adequate to use an effective theory to parametrize the low energy modifications to the SM. From an effective point of view, the situation here is very similar to the chiral symmetry breaking. There is a global group $\mathcal{G}$ that is broken to $\mathcal{H}$ due to the condensation of the fundamental degrees of freedom.

Due to the resemblance between the QCD and Composite Higgs effective descriptions, the techniques used in the construction of hadronic interactions are amply used in composite Higgs model building. One of the lessons we take here is the use of form factors to parametrize compositeness effects. Eventually, we will require the use of and other tools like the spectral decomposition, sum rules and large-N expansions.

These ideas from QCD corroborate to a scenario in which the resulting Hadrons are relatively stable and weakly interacting among themselves. The justification is due to 't Hooft, based on a perturbative expansion for the number of colours $N$ of a $S U(N)_{c}$ type theory. This expansion points to coherency between the microscopic asymptotically free theory, and the nonperturbative low energy regime of strongly interacting theories. Overall, it fits the hadron picture arising from QCD well.

When constructing the resonances of Composite Higgs theories, it is essential to know if their widths and interactions are controlled by the large-N limit or not.

All these questions must be taken into account when realizing the phenomenology we are interested. For now, we are interested in how to embed the SM consistently and the correct implementation of the misalignment mechanism. The nonperturbative intricacies of strongly interacting systems will be a topic for chapter 3. 


\subsubsection{Embeeding the Standard Model}

The EWSB is triggered by the presence of a strong sector that has itself a global SSB for generating a light Higgs. To guarantee this framework, we need to embed the SM group and provide particle representations according to the $\mathcal{G}$ symmetry.

As stated before, the new global symmetry breaking is like

$$
\mathcal{G} \rightarrow \mathcal{H} \supset S U(2)_{L} \times U(1)_{Y} \equiv \mathcal{G}_{E W}
$$

Thus the vacuum states are only invariant under a subgroup $\mathcal{H} \subset \mathcal{G}$, and the Goldstones transform as elements of the $\operatorname{coset} \mathcal{G} / \mathcal{H}$.

Defining $\left\{T^{A}\right\}$ as a basis of linearly independent generators for $\mathcal{G}$ with $A=$ $\{1,2, \ldots, \operatorname{dim}[\mathcal{G}]\}$, we can split the generators into two categories. There are the generators of the subgroup $\mathcal{H}$ which we design as unbroken generators $T^{a}$ and the ones of the coset $\mathcal{G} / \mathcal{H}$ which we call the broken $\hat{T}^{\hat{a}}$.

$$
\left\{T^{A}\right\}=\left\{T^{a}, \hat{T}^{\hat{a}}\right\}
$$

Together $\left\{T^{a}, \hat{T}^{\hat{a}}\right\}$ span the entire group $\mathcal{G}$. The reason we call them broken and unbroken generators is due to the spontaneous breaking pattern. The arbitrary reference vacuum $\vec{F}$ is defined as the direction in which the action of the unbroken and broken generators are:

$$
\begin{aligned}
& T^{a} \vec{F}=0 \\
& \hat{T}^{\hat{a}} \vec{F} \neq 0
\end{aligned}
$$

The coset must have at least four linearly independent elements to form a Higgs doublet. This relation defines the minimal number of coset elements needed to fully embed the Higgs, that is $\operatorname{dim}[\mathcal{G} / \mathcal{H}] \geq 4$. We also expect that $\operatorname{dim}[\mathcal{H}] \geq$ $\operatorname{dim}\left[\mathcal{G}_{E W}\right]=4$ since $\mathcal{H}$ must contain the Electroweak group. 


\begin{tabular}{ccccc}
\hline $\mathcal{G}$ & $\mathcal{H}$ & $C$ & $N_{G}$ & $\mathbf{r}_{\mathcal{H}}=\mathbf{r}_{\mathrm{SU}(2) \times \mathrm{SU}(2)}\left(\mathbf{r}_{\mathrm{SU}(2) \times \mathrm{U}(1)}\right)$ \\
\hline $\mathrm{SO}(5)$ & $\mathrm{SO}(4)$ & $\checkmark$ & 4 & $\mathbf{4}=(\mathbf{2}, \mathbf{2})$ \\
$\mathrm{SU}(3) \times \mathrm{U}(1)$ & $\mathrm{SU}(2) \times \mathrm{U}(1)$ & & 5 & $\mathbf{2}_{ \pm \mathbf{1} / \mathbf{2}}+\mathbf{1}_{\mathbf{0}}$ \\
$\mathrm{SU}(4)$ & $\mathrm{Sp}(4)$ & $\checkmark$ & 5 & $\mathbf{5}=(\mathbf{1}, \mathbf{1})+(\mathbf{2}, \mathbf{2})$ \\
$\mathrm{SU}(4)$ & {$[\mathrm{SU}(2)]^{2} \times \mathrm{U}(1)$} & $\checkmark^{*}$ & 8 & $(\mathbf{2}, \mathbf{2})_{ \pm \mathbf{2}}=2 \cdot(\mathbf{2}, \mathbf{2})$ \\
$\mathrm{SO}(7)$ & $\mathrm{SO}(6)$ & $\checkmark$ & 6 & $\mathbf{6}=2 \cdot(\mathbf{1}, \mathbf{1})+(\mathbf{2}, \mathbf{2})$ \\
$\mathrm{SO}(7)$ & $\mathrm{G}_{2}$ & $\checkmark^{*}$ & 7 & $\mathbf{7}=(\mathbf{1}, \mathbf{3})+(\mathbf{2}, \mathbf{2})$ \\
$\mathrm{SO}(7)$ & $\mathrm{SO}(5) \times \mathrm{U}(1)$ & $\checkmark^{*}$ & 10 & $\mathbf{1 0}_{\mathbf{0}}=(\mathbf{3}, \mathbf{1})+(\mathbf{1}, \mathbf{3})+(\mathbf{2}, \mathbf{2})$ \\
$\mathrm{SO}(7)$ & {$[\mathrm{SU}(2)]^{3}$} & $\checkmark^{*}$ & 12 & $(\mathbf{2}, \mathbf{2}, \mathbf{3})=3 \cdot(\mathbf{2}, \mathbf{2})$ \\
$\mathrm{Sp}(6)$ & $\mathrm{Sp}(4) \times \mathrm{SU}(2)$ & $\checkmark^{*}$ & 8 & $(\mathbf{4}, \mathbf{2})=2 \cdot(\mathbf{2}, \mathbf{2})$ \\
$\mathrm{SU}(5)$ & $\mathrm{SU}(4) \times \mathrm{U}(1)$ & $\checkmark^{*}$ & 8 & $\mathbf{4}-5+\overline{\mathbf{4}}+\mathbf{5}=2 \cdot(\mathbf{2}, \mathbf{2})$ \\
$\mathrm{SU}(5)$ & $\mathrm{SO}(5)$ & $\checkmark^{*}$ & 14 & $\mathbf{1 4}=(\mathbf{3}, \mathbf{3})+(\mathbf{2}, \mathbf{2})+(\mathbf{1}, \mathbf{1})$ \\
$\mathrm{SO}(8)$ & $\mathrm{SO}(7)$ & $\checkmark$ & 7 & $\mathbf{7}=3 \cdot(\mathbf{1}, \mathbf{1})+(\mathbf{2}, \mathbf{2})$ \\
$\mathrm{SO}(9)$ & $\mathrm{SO}(8)$ & $\checkmark$ & 8 & $\mathbf{8}=2 \cdot(\mathbf{2}, \mathbf{2})$ \\
$\mathrm{SO}(9)$ & $\mathrm{SO}(5) \times \mathrm{SO}(4)$ & $\checkmark^{*}$ & 20 & $(\mathbf{5}, \mathbf{4})=(\mathbf{2}, \mathbf{2})+(\mathbf{1}+\mathbf{3}, \mathbf{1}+\mathbf{3})$ \\
{$[\mathrm{SU}(3)]^{2}$} & $\mathrm{SU}(3)$ & & 8 & $\mathbf{8}=\mathbf{1}_{\mathbf{0}}+\mathbf{2}_{ \pm \mathbf{1}} \mathbf{2}+\mathbf{3}_{\mathbf{0}}$ \\
{$[\mathrm{SO}(5)]^{2}$} & $\mathrm{SO}(5)$ & $\checkmark^{*}$ & 10 & $\mathbf{1 0}=(\mathbf{1}, \mathbf{3})+(\mathbf{3}, \mathbf{1})+(\mathbf{2}, \mathbf{2})$ \\
$\mathrm{SU}(4) \times \mathrm{U}(1)$ & $\mathrm{SU}(3) \times \mathrm{U}(1)$ & & 7 & $\mathbf{3}_{-\mathbf{1} / \mathbf{3}}+\overline{\mathbf{3}}_{+\mathbf{1} / \mathbf{3}}+\mathbf{1}_{\mathbf{0}}=3 \cdot \mathbf{1}_{\mathbf{0}}+\mathbf{2} \pm \mathbf{1} / \mathbf{2}$ \\
$\mathrm{SU}(6)$ & $\mathrm{Sp}(6)$ & $\checkmark^{*}$ & 14 & $\mathbf{1 4}=2 \cdot(\mathbf{2}, \mathbf{2})+(\mathbf{1}, \mathbf{3})+3 \cdot(\mathbf{1}, \mathbf{1})$ \\
{$[\mathrm{SO}(6)]^{2}$} & $\mathrm{SO}(6)$ & $\checkmark^{*}$ & 15 & $\mathbf{1 5}=(\mathbf{1}, \mathbf{1})+2 \cdot(\mathbf{2}, \mathbf{2})+(\mathbf{3}, \mathbf{1})+(\mathbf{1}, \mathbf{3})$ \\
\hline
\end{tabular}

TABLE 2.1: Symmetry breaking patterns of different CHMs. The global group is $\mathcal{G} \rightarrow \mathcal{H}$. $C$ refers to having custodial symmetry, and $N_{G}$ is the number of coset elements. The last column contains the goldstone representations under $S O(4) \simeq S U(2)_{L} \times S U(2)_{R}$. The table was taken from [29].

These relations suggests that $\operatorname{dim}[\mathcal{G}]=\operatorname{dim}[\mathcal{H}]+\operatorname{dim}[\mathcal{G} / \mathcal{H}] \geq 8$. The $S U(3)$ group could be a fitting candidate for the minimal Composite Higgs model since its Lie Algebra has exactly 8 elements. However, there is a phenomenological problem in doing so - the resulting theory will violate custodial symmetry.

Before EWSB, the SM Higgs potential has a global $S U(2) \times S U(2)$. After EWSB, this is broken to $S U(2)_{c}$, making the remnant symmetry what is called custodial symmetry. If the Yukawa interactions coupled the same way in between families $^{3}$, the complete SM lagrangian would be custodial symmetric. Thus, the elementary sector alone has this $S U(2)_{c}$.

To ensure that the CHM has custodial symmetry, we need to ensure that the unbroken subgroup $\mathcal{H}$ contains a $S U(2)$ custodial. This forces $\mathcal{H}$ to have a dimension

\footnotetext{
${ }^{3}$ For example, if $y_{u}=y_{d}$ and hypercharge coupling were zero
} 
greater than 6 to reproduce the $S U(2) \times S U(2)$ structure. Then four generators are associated with the Electroweak group, and the other two remains due to custodial symmetry.

With the minimal coset to form a Higgs doublet, the smallest realization of a $\mathrm{CH}$ that respects custodial symmetry is $\mathcal{G}=S O(5)$ broken to $\mathcal{H}=S O(4)$. This relation defines the Minimal Composite Higgs Model (MCHM) [23].

We identify the Higgs and Gauge bosons electroweak representations by decomposing $\mathcal{H}$ into $S U(2)_{L} \times U(1)_{Y}$. Since $S O(4)$ is isomorphic to $S U(2)_{L} \times S U(2)_{R}$ the matching is direct - The $S U(2)_{L}$ from $S O(4)$ is associated with the weak isospin, and a $U(1)_{Y} \subset S U(2)_{R}$ is addressed to Hypercharge. With that, the Higgs has a $S O(4)$ representation that ensures its $\mathbf{2}_{ \pm \mathbf{1} / \mathbf{2}}$ under $\mathcal{G}_{E W}$ and Gauge bosons at their usual adjoint representations.

In contrast, there is much more freedom when identifying the $\mathcal{G}_{E W}$ representation for fermions. The simplest one defines the $\mathrm{MCHM}_{5}$, in which the flavour families of fermions are embeeded into 5's of $S O(5)$. This is done in such a way that the left-handed fermions transform as 4's of the unbroken $S O(4)$ and the right-handed ones as $\mathbf{1}^{\prime} s$.

$$
q_{L}^{\mathbf{4}}=\left(\begin{array}{c}
b_{l} \\
-i b_{l} \\
t_{l} \\
i t_{l} \\
0
\end{array}\right) \quad t_{R}^{\mathbf{1}}=\left(\begin{array}{c}
0 \\
0 \\
0 \\
0 \\
t_{r}
\end{array}\right) \quad b_{R}^{\mathbf{1}}=\left(\begin{array}{c}
0 \\
0 \\
0 \\
0 \\
b_{r}
\end{array}\right)
$$

There were proposed many other realizations that are different from the $\mathrm{MCHM}_{5}$, with non-minimal cosets and fermionic representations. Some of them are listed in table 2.1 .

Even though there are alternative CHM realizations, they all rely on the same theoretical structure. In all pNGB Higgs models, the misalignment of the order parameter $f$ occurs and generates EWSB. 


\subsubsection{Vacuum Misalignment}

When embedding the EW group into $\mathcal{H}$, the $S U(2)_{L} \times U(1)_{Y}$ must be formed as linear combinations of the unbroken generators.

$$
T_{E W}=\sum_{a} c_{a} T^{a}
$$

The SSB is given when there is a misalignment between the Higgs vacuum expectation value (vev) and the unbroken electroweak part of the generators. This relation is at the core of Composite Higgs models, EWSB is achieved through the condensation of the strong sector at TeV scales via the Vacuum Misalignment mechanism. The actual source of the vev misalignment is due to the explicit breaking of $\mathcal{G}$, that generates a potential and will be a theme for later on this chapter.

We start by defining the set of fields $\theta_{\hat{a}}(x)$ as the Goldstone modes. These fields span the degenerate vacua when $\mathcal{G}$ is not explicitly broken, thus transforming only with $\hat{T}^{\hat{a}}$ generators. The Higgs field can be written as

$$
\vec{\phi}=e^{\theta_{\hat{a}}(x) \hat{T}^{\hat{a}} \vec{F}}
$$

If we assume that the Higgs field gets a vev that is aligned with the reference vacuum, then $\langle\vec{\phi}\rangle=\vec{F}$ leads to only the breaking of the already broken generators $\hat{T}^{\hat{a}}$. This direction of alignment implies that the expectation value of the Goldstone mode is zero $\theta \equiv\left\langle\theta_{\hat{a}}\right\rangle=0$. In this case, there is no EWSB.

Now if we assume that the expectation value of the Goldstone mode is nonzero $\theta \equiv\left\langle\theta_{\hat{a}}\right\rangle \neq 0$, the Higgs vev gets misaligned with respect to the vacuum $\vec{F}$. In this case, the true vacuum direction $\langle\vec{\phi}\rangle$ gets a component into the unbroken EW generators, and EWSB is achieved. Figure 2.5 illustrates the whole process.

The amount of misalignment is given by the goldstone mode expectation value $\theta$ and defines the misalignment angle. Then the electroweak vev is obtained from the strong sector order parameter $f \equiv|\vec{F}|$ through $v=f \sin \theta$. Such a relationship 

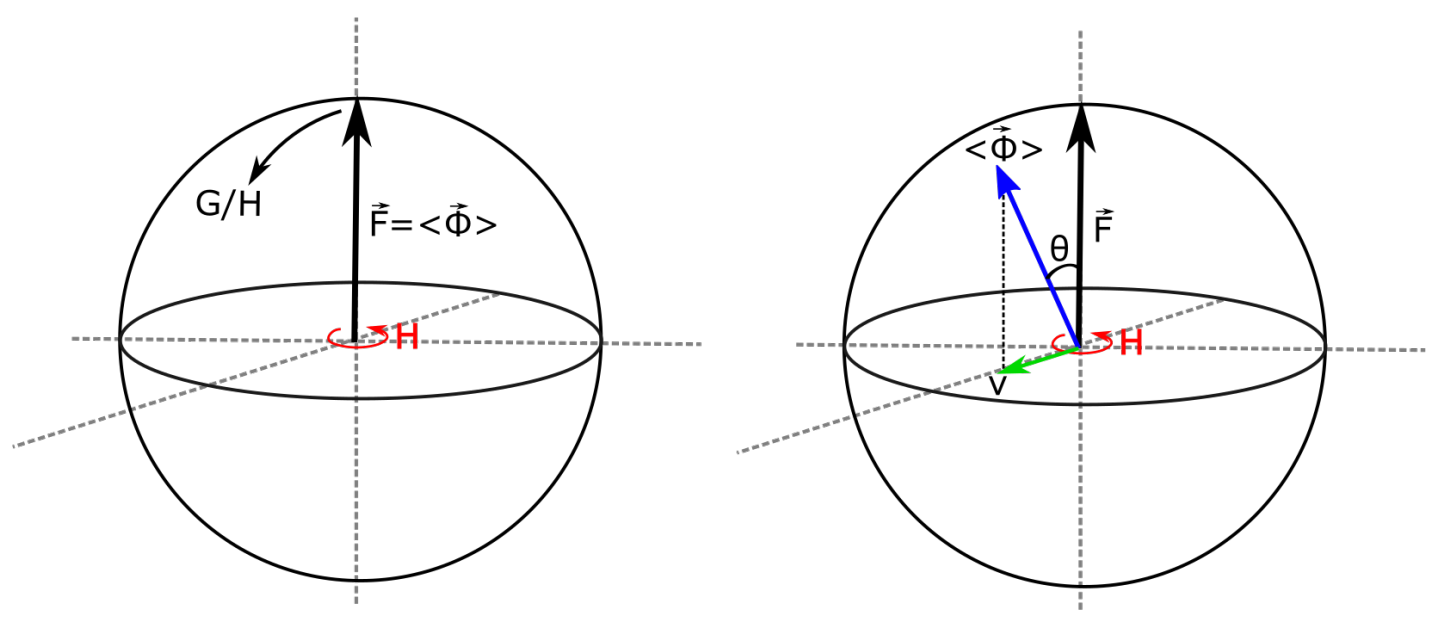

FIgURE 2.5: Misalignment mechanism. Reference vacuum $\vec{F}$ is defined such as it has no $\mathcal{H}$ component. If $\langle\vec{\phi}\rangle$ is aligned with $\vec{F}$, then there is no EWSB. Otherwise, if $\langle\vec{\phi}\rangle$ gets a non-zero goldstone expectation values, the small misalignment induces EWSB generating the EW vev $v$. Figure adapted from [33].

means that the electroweak scale is generated below $f$ if the misalignment angle is small enough.

At this level, we suppose that the misalignment angle can be naturally small, but this claim must be checked. When we proceed to calculate the Higgs potential generated from explicit $\mathcal{G}$ breakings, we shall address this question. For now, we can quantify the tuning in adjusting $\theta$ by the parameter

$$
\xi \equiv \frac{v^{2}}{f^{2}}
$$

this shall be an important quantifier of the SM deviations later on.

In this kind of model, we expect to recover the SM by sending the composite sector to very high scales. In such scenario, the effects of the new strong sector are diminished as $f \rightarrow \infty$, or $\xi \rightarrow 0$. The BSM physics is effectively decoupled in such a limit. This is the main feature of CHM; tuning is reintroduced as the deviations from SM couplings are reduced experimentally.

To compute the contributions to Higgs couplings, we must know how to 
parametrize the low energy degrees of freedom effectively. It will be necessary to comprehend the non-linear realization of the Higgs field and the CCWZ construction.

\subsection{Non-linearly Realized Symmetries}

Given the general context of CHMs, we want to find a systematic way to construct and predict the model's features. This is mainly done in the context of the Callan-Coleman-Wess-Zumino (CCWZ) construction $[59,58]$. We are mainly concerned with a non-linear parameterization of the Higgs, that is directed for theories with built-in global SSB patterns. These are key to determining the modifications to the SM couplings when a new strong sector is present.

The low energy degrees of freedom of CHM are just the known elementary particles and a composite Higgs. To write down the NGB's Lagrangian, we start from a scalar field $\Sigma$ that transforms linearly with respect to the full $\mathcal{G}$ group.

$$
\Sigma \rightarrow g \Sigma \quad \text { with } g \in \mathcal{G}
$$

Now we define a new representation that divides the NGB's that are parametrize the coset $\mathcal{G} / \mathcal{H}$, together with the remaining non-goldstone fields.

$$
\Sigma(x) \equiv U[\pi(x)] \Sigma_{0}(x)
$$

Built to be invariant under $h \in \mathcal{H}$ transformations, $\Sigma_{0}(x)$ contains the vacuum $\vec{F}$ and massive excitations around it. The other object, $U[\pi(x)]$ is called the Goldstone matrix and is constructed solely from goldstone fields. We can see how it transforms by acting a $\mathcal{G}$ transformation on the $\Sigma$ field.

$$
g \Sigma(x)=g U[\pi(x)] \Sigma_{0}(x)=U\left[\pi^{\prime}(x)\right] h[\pi, g] \Sigma_{0}=U\left[\pi^{\prime}(x)\right] \Sigma_{0}
$$


Hence, the NGB transformation induce the goldstone matrix to transform as

$$
U[\pi] \rightarrow g U[\pi] h^{\dagger}[g, \pi]
$$

Relation (2.14) obeys the group multiplication law, with $h$ being local since it contains the NGB fields. Because of this structure, $U[\pi]$ is a non-linear realization of $\mathcal{G}$.

More concretelly, we could write a generic group element $g$ as

$$
g=\exp \left(i \alpha_{A} T^{A}\right)=\exp \left(i f_{\hat{a}}[\alpha] T^{\hat{a}}\right) \exp \left(i f_{a}[\alpha] T^{a}\right)
$$

In which $f_{a}$ and $f_{\hat{a}}$ are functions of the $\alpha$ parameters. Up to $\mathcal{O}(\xi)$ they satisfy:

$$
\begin{aligned}
& f_{\hat{a}}=\alpha_{\hat{a}}+\mathcal{O}\left(\alpha^{2}\right), \\
& f_{a}=\alpha_{a}+\mathcal{O}\left(\alpha^{2}\right) .
\end{aligned}
$$

Since $T^{a}$ generators leave $\Sigma_{0}$ invariant, the scalar field can be written in the non-linear realization $a^{4}$ :

$$
\begin{aligned}
\phi(x)=\exp \left(i \theta_{A} T^{A}\right) \Sigma_{0} & =\exp \left(\frac{i \sqrt{2}}{f} \pi_{\hat{a}} T^{\hat{a}}\right) \exp \left(\frac{i \sqrt{2}}{f} \xi_{a} T^{a}\right) \Sigma_{0} \\
& =\exp \left(\frac{i \sqrt{2}}{f} \pi_{\hat{a}} T^{\hat{a}}\right) \Sigma_{0}
\end{aligned}
$$

We can see that the Goldstone matrix is

$$
U[\pi]=\exp \left(\frac{i \sqrt{2}}{f} \pi_{\hat{a}} T^{\hat{a}}\right)
$$

Neglecting possible heavy excitations, $\Sigma_{0}$ is just the reference vacuum $\Sigma_{0}=\vec{F}$.

${ }^{4}$ The factor of $\frac{\sqrt{2}}{f}$ was included to canonically normalize the kinetic terms in the upcoming effective lagrangian construction. 
Relying on representation independence we choose to use the non-linear approach for the Higgs since it is more suitable to describe the global symmetry breaking. The Higgs field is then obtained from the goldstone $\pi_{\hat{a}}$ 's that span the coset $\mathcal{G} / \mathcal{H}$

$$
\Sigma(x)=\exp \left(\frac{i \sqrt{2}}{f} \pi_{\hat{a}} T^{\hat{a}}\right) \vec{F}
$$

For the Gauge boson representations, we have to correctly match the gauged generators with the strong sector unbroken generators. The actual process is straightforward; the Gauge boson fields transform as the usual adjoint representation of the EW group and are formed as a linear combination of $\mathcal{H}$ elements.

As we have discussed, gauging a subgroup of an exact global symmetry breaks the given symmetry explicitly. This will induce a potential for the Higgs making it massive, but still light compared to the rest of the composite sector.

The non-linear realization for the fermionic fields is similar to the Higgs. We define the multiplets with respect to representations of $\mathcal{G}$ and dress the fermion fields with the Goldstone matrix to get a non-linear transformation law.

$$
Q_{l}^{[\mathbf{r}]} \equiv U[\pi] q_{l} \quad T_{r}^{[\mathbf{r}]} \equiv U[\pi] t_{r}
$$

where the $[\mathbf{r}]$ index refers to the fermionic representation. Both $q_{l}$ and $t_{r}$ are linearly realized and are the simple immersions of the standard model $S U(2)_{L}$ multiplets into $\mathcal{H}$ multiplets. It often happens that the SM fermions fit loosely to these new $\mathcal{G}$ representations - so to say, they form incomplete multiplets. These sources of explicit breaking will be essential when combining with the gauged contributions to the potential to the Higgs.

Such prescription comes in handy when building the effective lagrangian of these low energy degrees of freedom. After we fix a specific CHM group, the Higgs 
and gauge boson interactions will come from their kinetic terms.

$$
\begin{gathered}
\mathcal{L}_{\text {gauge }}=\frac{1}{2}\left(D_{\mu} \Sigma\right)^{\dagger} D^{\mu} \Sigma-\frac{1}{4} A_{\mu \nu}^{i} A^{i \mu \nu} \\
D_{\mu} \equiv \partial_{\mu}-i A_{\mu}^{i} T^{i}, \quad T^{i} \in \mathcal{G}_{E W}
\end{gathered}
$$

Additionally, the fermion mass terms and interactions will come from the possible $\mathcal{G}$ invariant contraction.

$$
\mathcal{L}_{\text {fermion }}=m_{*} \overline{Q_{l}^{[\mathbf{r}]}} T_{r}^{[\mathbf{r}]}+\text { h.c. }
$$

With these simple, effective terms, we shall proceed to choose a specific realization of CHMs, the $\mathrm{MCHM}_{\mathbf{5}}$, and reobtain the SM with its misaligned contributions to the couplings of the Higgs.

\subsection{Composite Higgs couplings in the MCHM5}

The SM has precise predictions on the Higgs couplings. The fermions Yukawa couplings are proportional to their masses, and the gauge-Higgs couplings go with the massive bosons masses squared. In a composite Higgs model, these couplings are generally modified due to the misalignment of the electroweak scale.

We wish to calculate the $\mathrm{MCHM}_{5}$ Higgs interactions modifications at the level of the effective local theory. In such, the internal dynamics are completely integrated out, without any momentum dependent non-local effects. This defines the model at zero transferred momentum.

The program is to expand the effective Lagrangian expressions in the nonlinear realization and compute the Feynman rules of the model.

Remember that in the $\mathrm{MCHM}_{5}, \mathcal{G}$ is the $S O(5)$ group, and the fermions are embeeded in 5's representations. Then, the $S O(5)$ is spontaneously broken to $\mathcal{H}=$ 
$S O(4)$ and the Higgs is realized though the non-linear $\Sigma$ field.

$$
\begin{array}{r}
\Sigma(x)=e^{i \frac{1}{f} \Pi_{i}(x) \hat{T}^{i}} \Sigma_{0} \\
\Sigma_{0}=\vec{F}
\end{array}
$$

The generators of $S O(5)$ are divided between broken $T^{\hat{a}}$ and unbroken $T^{a}$, as $T^{A}=\left\{T^{a}, T^{\hat{a}}\right\}$. We can define $T_{L}^{a}$ and $T_{R}^{a}$ generators corresponding to $S O(4) \simeq$ $S U(2)_{L} \times S U(2)_{R} \simeq S O(4)$, just for computation convenience.

$$
\begin{aligned}
{\left[T_{L, R}^{a}\right] } & =-\frac{i}{2}\left[\frac{1}{2} \epsilon^{a b c}\left(\delta_{i}^{b} \delta_{j}^{c}-\delta_{j}^{c} \delta_{i}^{b}\right) \pm\left(\delta_{i}^{a} \delta_{j}^{4}-\delta_{j}^{a} \delta_{i}^{4}\right)\right] \\
{\left[T^{\hat{a}}\right] } & =-\frac{i}{\sqrt{2}}\left(\delta_{i}^{\hat{a}} \delta_{j}^{5}-\delta_{j}^{\hat{a}} \delta_{i}^{5}\right)
\end{aligned}
$$

These generators have the following structure,

$$
T^{A}=\left(\begin{array}{c|c} 
& \\
\mathcal{H} & \mathcal{G} / \mathcal{H} \\
\hline \mathcal{G} / \mathcal{H} &
\end{array}\right)
$$

so the vacuum that satisfies the properties of (2.14) can be defined as:

$$
\vec{F}=(0,0,0,0, f)^{T}
$$

With $i, j=1, \ldots 5$. Using these definitions for the generators, $\Sigma(x)$ assume the form:

$$
\Sigma(x)=f\left(\begin{array}{c}
\operatorname{Sin}\left(\frac{\Pi}{f}\right) \hat{\Pi} \\
\operatorname{Cos}\left(\frac{\Pi}{f}\right)
\end{array}\right)
$$


Here $\Pi$ is the norm of the NGB vector $\vec{\pi}(x)$, that can be expressed in terms of the Higgs doublet components.

$$
\vec{\pi}=\left(\begin{array}{c}
\pi_{1} \\
\pi_{2} \\
\pi_{3} \\
\pi_{4}
\end{array}\right)=\frac{1}{\sqrt{2}}\left(\begin{array}{c}
-i\left(h_{u}-h_{u}^{\dagger}\right) \\
h_{u}+h_{u}^{\dagger} \\
i\left(h_{d}-h_{d}^{\dagger}\right) \\
h_{d}+h_{d}^{\dagger}
\end{array}\right)
$$

As in the SM, we define the unitary gauge as a specific direction in which the Higgs mode oscillates. Here we choose $\pi_{4}$ as the Higgs mode, and the other components get absorbed by the massive gauge bosons.

Furthermore, we divide the fermion 5-plets into a fourplet for the left handed fermions and a singlet for the right handed fields.

$$
\begin{aligned}
q_{L}^{\mathbf{4}} & =\left(0,0, t_{l}, i t_{l}, 0\right)^{T} \\
t_{R}^{\mathbf{1}} & =\left(0,0,0,0, t_{r}\right)^{T}
\end{aligned}
$$

where the bottom fields were ommited since they do not take an important role in the following discussions.

\subsubsection{Gauge Boson Interactions}

To compute the gauge boson interactions we start with

$$
\mathcal{L}_{\text {gauge }}=\frac{1}{2}\left(D_{\mu} \Sigma\right)^{\dagger} D^{\mu} \Sigma-\frac{1}{4} A_{\mu \nu}^{i} A^{i \mu \nu}
$$

Using (2.30) and the explicit generators we arrive at

$$
\begin{aligned}
\mathcal{L}_{\text {gauge }}= & \frac{1}{2}\left(D_{\mu} \Sigma\right)^{\dagger} D^{\mu} \Sigma-\frac{g^{2}}{4} f^{2} W_{\mu}^{+} W^{-, \mu} \sin ^{2}\left(\frac{h}{f}\right)+\frac{g^{2}}{8 c_{W}^{2}} f^{2} Z_{\mu} Z^{\mu} \sin ^{2}\left(\frac{h}{f}\right) \\
& -\frac{1}{4} W_{\mu \nu}^{i} W^{i \mu \nu}-\frac{1}{4} B_{\mu \nu}^{i} B^{i \mu \nu}
\end{aligned}
$$


When the Higgs assume its vev, $h$ gets shifted by $h \rightarrow v+h$ so

$$
\begin{aligned}
f^{2} \sin \left(\frac{v+h}{f}\right) \simeq f^{2} & {\left[\sin ^{2}\left(\frac{v}{f}\right)+2 \cos \left(\frac{v}{f}\right) \sin \left(\frac{v}{f}\right) \frac{h}{f}\right.} \\
& \left.+\left(\cos ^{2}\left(\frac{v}{f}\right)-\sin ^{2}\left(\frac{v}{f}\right)\right) \frac{h^{2}}{f^{2}}+\mathcal{O}\left(h^{3}\right)\right] \\
\approx & v^{2}+2 v \sqrt{1-\frac{v^{2}}{f^{2}}} h+\left(1-\frac{2 v^{2}}{f^{2}}\right) h^{2}+f^{2} \mathcal{O}\left(h^{3}, v^{4} / f^{4}\right)
\end{aligned}
$$

In (2.36) we are expanding for $h / f$ and ignoring the 4-higgs term that will appear as effective 6-point operators. (2.37) assumes that the misalignment angle is small so it is reasonable to truncate terms higher than $\xi^{2}=v^{4} / f^{4}$. Substituting back to 2.36 lead to

$$
\begin{aligned}
\mathcal{L}_{\text {gauge }}= & \frac{1}{2}\left(D_{\mu} \Sigma\right)^{\dagger} D^{\mu} \Sigma-\frac{1}{4} W_{\mu \nu}^{i} W^{i \mu \nu}-\frac{1}{4} B_{\mu \nu}^{i} B^{i \mu \nu} \\
& +2 m_{w}^{2} W_{\mu}^{+} W^{-, \mu}+\frac{2 i m_{w}^{2}}{v} \sqrt{1-\xi} W_{\mu} W^{\mu} h+\frac{m_{z}^{2}}{v} Z_{\mu} Z^{\mu} \\
& +\frac{2 i m_{z}^{2}}{v} \sqrt{1-\xi} Z_{\mu} Z^{\mu} h+\frac{2 i m_{w}^{2}}{v^{2}}(1-2 \xi) W_{\mu} W^{\mu} h^{2}+\frac{2 i m_{z}^{2}}{v^{2}}(1-2 \xi) Z_{\mu} Z^{\mu} h^{2}
\end{aligned}
$$

Where $m_{W}=c_{w} m_{Z}, c_{w}$ is the Weinberg angle and $\xi=v^{2} / f^{2}$. Then, the $h V V$ deviations with respect to the SM $g_{h V V}^{S M}=\frac{2 i m_{V}^{2}}{v}$ can be defined as

$$
\kappa_{V}=\frac{g_{h V V}^{C H}}{g_{h V V}^{S M}}=\sqrt{1-\xi}
$$

Also, there will be modifications to the two Higgs vertices.

$$
\kappa_{V}=\frac{g_{h h V V}^{C H}}{g_{h h V V}^{S M}}=\sqrt{1-\xi}
$$

To summarize, in the $\mathrm{MCHM}_{5}$ the $\mathrm{SM}$ couplings always get suppressed with the $\kappa_{V}$ factors. Additionally, custodial symmetry is respected at tree level by construction. As we argued before, these models with a pNGB Higgs have an SM limit. Here we can explicitly check that by making $\xi \rightarrow 0$ all couplings return to the SM. 


\subsubsection{Fermion Interactions}

The fermions interactions with the Higgs do not only depend on the choice of symmetry breaking pattern but also on the specific immersion of the fermions into $\mathcal{G}$ representations. In the UV, $S O(5)$ is unbroken, and particles are grouped as multiplets in the $\mathcal{G}$-representation.

Accordingly, there is some degree of ambiguity in choosing $S O(5)$ representations, but they must contain the $\mathcal{G}_{E W}$ quantum numbers of the left-handed doublets of $S U(2)_{L}$ and right-handed singlets with the correct hypercharges. What we need are a $\mathcal{G}_{E W} \mathbf{2}_{1 / 6}$ for $q_{l}$, a $\mathbf{1}_{2 / 3}$ for $t_{r}$ and a $\mathbf{1}_{-1 / 3}$ for $b_{r}$.

However this representation do not exist for the previously chosen 5 of $S O(5)$. A five decomposes as $\mathbf{5} \rightarrow \mathbf{4} \oplus \mathbf{1}=(\mathbf{2}, \mathbf{2}) \oplus \mathbf{1}$. Under $\mathcal{G}_{E W}$ this is $\mathbf{2}_{1 / 2} \oplus \mathbf{2}_{-1 / 2} \oplus \mathbf{1}$, which does not contain th necessary quantum numbers for the fermions.

As a result, it is necessary to postulate the existence of another auxiliary $U(1)_{X}$ group which the only role is to provide the correct hypercharges to the fermions. Since $U(1)_{X}$ and $S O(5)$ generators do commute, this auxiliary group does not alters or participates in the SSB of $\mathcal{G}$ nor alter the details in the CCWZ construction. ${ }^{5}$

$$
S O(5) \times U(1)_{X} \rightarrow S O(4) \times U(1)_{X}
$$

All fermions will have charges under this $U(1)_{X}$. Accordingly, this choice is made such that all SM hypercharges are fixed to their usual attributes. Furthermore, the gauge bosons and the Higgs are neutral in $X$.

$$
Y \equiv T_{R}^{3}+X
$$

We will adress only Top-Higgs interactions since they will be the ones with leading phenomenologial effects. Using the effective description with dressed Quark

\footnotetext{
${ }^{5}$ Traditionally, the extra $U(1)_{X}$ is often omitted since it does not play major roles in the effective description.
} 
fields we can compute the yukawa interactions.

$$
\begin{aligned}
& \mathcal{L}_{\text {fermion }}=m_{*} \overline{Q_{l}^{[\mathbf{5}]}} T_{r}^{[\mathbf{5}]}+\text { h.c. } \\
& Q_{l}^{[\mathbf{5}]}=U[\pi] q_{l} \quad T_{r}^{[\mathbf{5}]}=U[\pi] t_{r}
\end{aligned}
$$

Because of the presence of the Goldstone matrix, these terms will induce Higgs interactions. We can compute them explicitly

$$
\begin{aligned}
& Q_{l}^{[\mathbf{5}]}=\left(e^{i \sqrt{2} \hat{T}^{\hat{a}} \pi^{\hat{a}}(x) / f}\right)\left(\begin{array}{c}
0 \\
0 \\
-i t_{L} \\
t_{L} \\
0
\end{array}\right)=t_{L}\left(\begin{array}{c}
\pi_{1}\left(\pi_{4}-i \pi_{3}\right)\left(\cos \frac{|\pi|}{f}-1\right) \\
\pi_{2}\left(\pi_{4}-i \pi_{3}\right)\left(\cos \frac{|\pi|}{f}-1\right) \\
\frac{-1}{|\pi|^{2}}\left[i \pi_{1}^{2}-i \pi_{2}^{2}+\left(\pi_{3} \cos \frac{|\pi|}{f}-i \pi_{4}\right)\left(\pi_{3}+i \pi_{4}\right)\right] \\
\frac{1}{|\pi|^{2}}\left[\pi_{1}^{2}+\pi_{2}^{2}-\left(i \pi_{4} \cos \frac{|\pi|}{f}-\pi_{3}\right)\left(\pi_{3}+i \pi_{4}\right)\right] \\
\frac{1}{|\pi|^{2}} \sin \frac{|\pi|}{f}\left(\pi_{3}+i \pi_{4}\right)
\end{array}\right) \\
& T_{r}^{[\mathbf{5}]}=\left(e^{i \sqrt{2} \hat{T}^{\hat{a}} \pi^{\hat{a}}(x) / f}\right)\left(\begin{array}{c}
0 \\
0 \\
0 \\
0 \\
t_{R}
\end{array}\right)=t_{R}\left(\begin{array}{c}
\pi_{1} \frac{\sin \frac{|\pi|}{f}}{|\pi|} \\
\pi_{2} \frac{\sin \frac{|\pi|}{f}}{|\pi|} \\
\pi_{3} \frac{\sin \frac{|\pi|}{f}}{|\pi|} \\
\pi_{4} \frac{\sin \frac{|\pi|}{f|\pi|}}{\cos \frac{|\pi|}{f}}
\end{array}\right)
\end{aligned}
$$

In the unitary gauge, we define $\pi_{4}(x)=h(x)$ and this expression simplifies to

$$
Q_{l}^{[\mathbf{5}]}=q_{l}\left(\begin{array}{c}
0 \\
0 \\
0 \\
\cos (h / f) \\
\sin (h / f)
\end{array}\right) T_{r}^{[\mathbf{5}]}=t_{r}\left(\begin{array}{c}
0 \\
0 \\
0 \\
\sin (h / f) \\
\cos (h / f)
\end{array}\right)
$$


Finally we can obtain the fermion mass terms and yukawa interactions via the effective lagrangian for dressed fields.

$$
\begin{aligned}
\mathcal{L}_{\text {fermion }} & =m_{*} \overline{Q_{l}^{[5]}} T_{r}^{[\mathbf{5}]}+\text { h.c. } \\
& =2 m_{*} \overline{t_{L}} t_{R} \cos \left(\frac{h}{f}\right) \sin \left(\frac{h}{f}\right)+\text { h.c. } \\
& =m_{*} \overline{t_{L}} t_{R} \sin \left(\frac{2 h}{f}\right)+\text { h.c. }
\end{aligned}
$$

Again, when the Higgs assume its vev, $h$ gets shifted by $h \rightarrow v+h$ and $\sin \left(\frac{2 h}{f}\right) \rightarrow \sin \left(\frac{2(v+h)}{f}\right)$. We can define the top-mass by expanding the higgses interactions and kepping lowest order of $\xi$.

$$
\begin{aligned}
\mathcal{L}_{\text {fermion }} & =\frac{m_{*}}{f} v \sqrt{1-\xi} \bar{t}_{L} t_{R}+m_{*} \frac{2}{f}(1-2 \xi) h \overline{t_{L}} t_{R}+\text { h.c. } \\
\Rightarrow m_{t} & \equiv \frac{m_{*}}{f} v \sqrt{1-\xi} \\
\mathcal{L}_{\text {fermion }} & =m_{t} \overline{t_{L}} t_{R}+\frac{\sqrt{2} m_{t}}{v}\left[\frac{1-2 \xi}{\sqrt{1-\xi}}\right] h \overline{t_{L}} t_{R}+h . c .
\end{aligned}
$$

Concearning the SM interactions we get a suppression of the fermion couplings that goes like a function of the misalignment angle $\xi$.

$$
k_{t}^{\mathbf{5}} \equiv \frac{g_{h t t}^{C H}}{g_{h t t}^{S M}}=\frac{1-2 \xi}{\sqrt{1-\xi}} \approx 1-\frac{3}{2} \xi
$$

These suppressions to the SM scale to the square in the cross-section and distribution functions. Because of the non-dynamical shift in the interactions, the momentum flow in the precesses is not altered compared to SM. Because of this, we do not expect differences in the shape of cross-sections and kinematic distributions here. The modification is just an overall normalization suppression in all observables. 


\subsection{Interactions between Elementary and Compos- ite Sectors}

We computed the modifications to SM couplings in the CHM framework via the effective field theory of the low energy degrees of freedom. These degrees of freedom are the same particles as in the standard model and get a misalignment suppression in their interactions.

Even though these modifications are a direct consequence of the presence of the strong sector, it does not suggest any experimental feature of compositeness itself. Here, no "smoking gun" of compositeness is available, and there are no kinematic departures from elementarity. To provide a structure that alters the momentum flow, we must keep the influence of the strong sector interactions to a higher degree than presented before.

As we have defined, the theory associated with a composite Higgs should have an elementary sector, a composite one, and interactions between the two of them. We can integrate out $\mathcal{L}_{C S}$ and $\mathcal{L}_{\text {int }}$, but this time keeping momentum dependence through the non-local prescription of form factors.

The idea is to understand how these interactions allow the CHM to becomes natural. As we will see, the most consistent way to realize a CHM that addresses the HP will be to introduce mixings between the elementary and composite particles.

\subsubsection{Composite and Elementary Mixing}

An important feature of the interactions between the composite and elementary sectors is the introduction of mixings. These mixings are phenomenologically attractive because they fit the internal structure of the theory and provide an explicit way of dealing with the hierarchy problem. In the following, we shall describe how these interactions come to be.

The gauge bosons were previously introduced as external sources of a part of the global symmetry group. Assuming the existence of resonances that have 
the same quantum numbers of elementary gauge bosons, the external source terms couples them linearly. These interactions predict mixings between the two vector particles.

Such resonances are generally named as vector partners and are important signals of CHMs. The mixing interaction is a straightforward way of modelling $\mathcal{L}_{\text {int }}$ and is similar to the hadronic interactions with electromagnetic currents back in QCD. Such prescription was framed in vector-meson dominance with the $\rho$ meson mixing directly with the photon.

More concretelly, we can write this gauge interactions with their vector-partners.

$$
\mathcal{L}_{\text {int }}^{\text {vector }}=J^{a \mu} W_{\mu}^{a}+J_{Y}^{\mu} B_{\mu}
$$

The fermions also get a mixing-type of interaction, though it is more difficult to visualize why it is the case. Now, the principal motivation of mixing interactions comes from the necessity to avoid Yukawa couplings in the fermion sector [28].

The central point is that, within the usual bilinear interactions, we cannot expect the generation of a Yukawa coupling for the top-quark that is realistic to its experimental value and at the same time does not suffers from the hierarchy problem. The argument for this remark comes from the scaling dimensions of the operators that generate the fermion masses.

There are mainly two forms of coupling elementary fermions to the composite sector. Either through a linear interaction or a bilinear one.

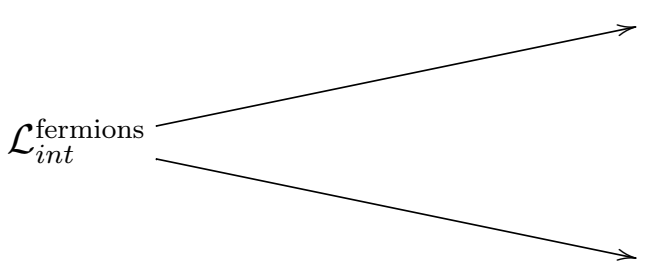

Bilinears (technicolor)

Linear (partial compositeness) 
If we use bilinear interactions, the lagrangian is given by:

$$
\mathcal{L}_{\text {int }}^{\text {bilinear }}=\frac{\lambda_{t}}{\left(\Lambda_{U V}\right)^{d-1}} \bar{q}_{L} \mathcal{O}_{S}^{c} t_{R}+\text { h.c. }
$$

where $\mathcal{O}_{s}^{c}$ is a Lorentz scalar operator with mass dimension $d, \lambda_{t}$ is the coupling of the interaction term and $\Lambda_{U V}$ the ultraviolet cutoff. This scalar operator has the same quantum numbers as the Higgs and is analogous to the Higgs Yukawa interaction in the SM.

By scaling such operators from $\Lambda_{U} V$ to the ressonance mass scale $m_{*}$ the effective coupling $\lambda_{t}$ evaluated at IR scales should be

$$
\begin{aligned}
\mathcal{L}_{\text {int }}^{\text {bilinear }}\left[\Lambda_{U V}\right] & =\frac{\lambda_{t}}{\left(\Lambda_{U V}\right)^{d-1}} \bar{q}_{L} \mathcal{O}_{s}^{c} t_{R} \rightarrow \mathcal{L}_{\text {int }}^{\text {bilinear }}\left[m_{*}\right]=\frac{\lambda_{t}\left[m_{*}\right]}{m_{*}^{d-1}} \bar{q}_{L} \mathcal{O}_{s}^{c} t_{R} \\
\Rightarrow \lambda_{t}\left[m_{*}\right] & =\left(\frac{m_{*}}{\Lambda_{U V}}\right)^{d-1} \lambda_{t}
\end{aligned}
$$

This relation suggests that if the operator is not close to being marginal, with $d=1$, then it is hard to generate large Yukawa couplings for the top-quark. This is simply the Wilsonian statement that we are working with effective field theory. It is a difficult task to postulate a set of interactions at the UV, run them down to the $m_{*}$ scale and get a parametrically large coefficient if $d \neq 1$, since the operator is suppressed by large scales.

If we choose $d=1$, the generation of the Yukawa couplings is just as in the SM, recovering the HP. The actual dynamical generation is portrayed in the left panel of figure 2.6.

Alternatively, one could try to use a linear coupling that assumes the form:

$$
\mathcal{L}_{\text {int }}^{\text {linear }}=\frac{\lambda_{t_{L}}}{\left(\Lambda_{U V}\right)^{d_{L}-5 / 2}} \bar{q}_{L}^{[\mathbf{5}]} \mathcal{O}_{F}^{L}+\frac{\lambda_{t_{R}}}{\left(\Lambda_{U V}\right)^{d_{R}-5 / 2}} \bar{t}_{R}^{[\mathbf{5}]} \mathcal{O}_{F}^{R}
$$

Now, the operator $\mathcal{O}_{F}$ which couples with fermions is not scalar anymore, but fermionic. Here the required dimension for the interaction to be marginal is $d=5 / 2$. 

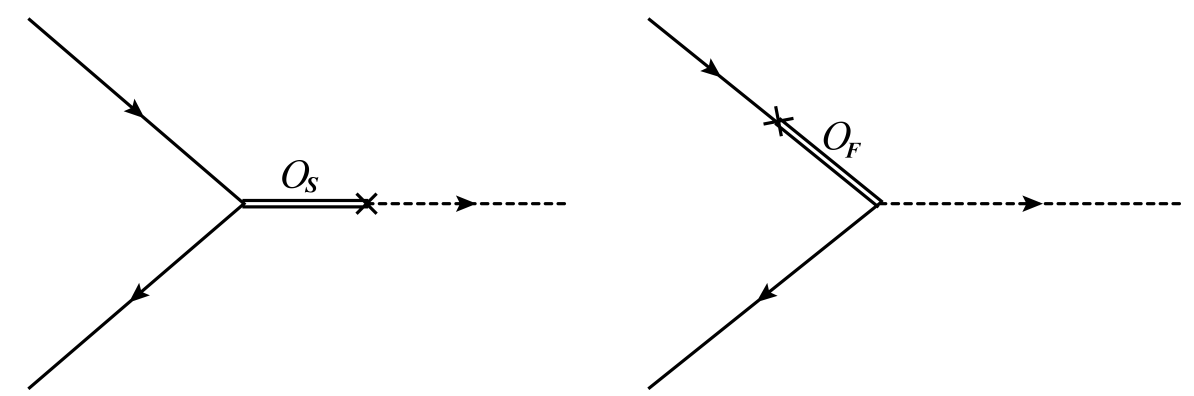

FIgURE 2.6: Generation of composite Higgs couplings. We divided between the case of a bilinear (left) and a (linear) structure of fermion couplings with UV composite operators. By using a scalar $\mathcal{O}_{S}$ we reitroduce the HP back into the model. Thus, the preferred way to contruct a CHM is to use PC.

Thus the scaling of the coupling parameters is

$$
\lambda_{t_{L}}\left[m_{*}\right]=\left(\frac{m_{*}}{\Lambda_{U V}}\right)^{d-5 / 2} \lambda_{t}
$$

and obtaining a $\mathcal{O}(1)$ contribution for the Yukawa coupling dynamically is feasible for an approximately marginal operator. In such a case, the HP is not reintroduced in the theory.

The $d=5 / 2$ fermionic operators are assumed to create particle states that have the same quantum numbers as the elementary fermions. We can assign to this operator a field that correspond to a resonant single particle-state denoted as the fermion partner.

$$
\begin{aligned}
& \left\langle 0\left|\mathcal{O}_{F}^{L}\right| \tilde{Q}\right\rangle \neq 0 \\
& \left\langle 0\left|\mathcal{O}_{F}^{L}\right| \tilde{T}\right\rangle \neq 0
\end{aligned}
$$

Because of the goldstone matrix in $\bar{q}_{L}^{[\mathbf{5}]}$ in (2.57), the Yukawa couplings can be dynamically generated through $\mathcal{O}_{F}^{L}$. In this case, when the Higgs assumes its vev, there will be mixings between the elementary top-quark and its heavy partner. Because of these mixings, such implementation of linear couplings is called Partial Compositeness (PC). 
The generation of the Yukawa coupling is depicted in the right panel of figure 2.6. The explicit implementation of PC scenario, with physical resonances, will be achieved in chapter 4 .

\subsubsection{The Composite Hierarchy Solution}

With the assumed interactions via external gauge sources and partial compositeness, the Lagrangian at the compositeness scale is

$$
\mathcal{L}=\mathcal{L}_{C S}+\mathcal{L}_{E S}+J^{a \mu} W_{\mu}^{a}+J_{Y}^{\mu} B_{\mu}+\sum \lambda_{r} \bar{\psi}_{r} \mathcal{O}_{r}+\text { h.c. }
$$

The details of the Composite Sector (CS) are, in general, not known and unimportant for the following discussion. We are aiming to obtain an effective description in which the CS degrees of freedom are not explicitly written, but their dynamical effects still present. To reach our objective, we need a description that includes momentum dependence.

Now, we write an effective Lagrangian that respect $\mathcal{G}$ symmetry, but with the resonances integrated-out. We proceed to parametrize their contributions through the momentum dependent coeffiecients $\Pi\left(p^{2}\right)$ and $M\left(p^{2}\right)$ - these will be generically denoted as our form factors [23, 34].

$$
\begin{aligned}
\mathcal{L}_{\text {eff }}^{\mathcal{G}}= & \frac{1}{2} \mathcal{P}^{\mu \nu}\left(\Pi_{0}(p) \operatorname{Tr}\left(A_{\mu} A_{\nu}\right)+\Pi_{1}(p) \Sigma^{T} A_{\mu} A_{\nu} \Sigma\right)+ \\
& +\overline{Q_{L}} \not p\left(\Pi_{0}^{L}(p)+\Pi_{1}^{L}(p) \Sigma \Sigma^{T}\right) Q_{L}+ \\
& +\overline{T_{R}} \not p\left(\Pi_{0}^{R}(p)+\Pi_{1}^{R}(p) \Sigma^{T} \Sigma\right) T_{R}+ \\
& +\overline{Q_{L}}\left(M_{0}(p)+M_{1}(p) \Sigma \Sigma^{T}\right) T_{R}
\end{aligned}
$$

where $\mathcal{P}_{T}^{\mu \nu}=g^{\mu \nu}-p^{\mu} p^{\nu} / p^{2}$, is the transverse polarization projector.

In (2.62) we retained terms up to order $\Sigma^{2}$ and built the lagrangian to respect $\mathcal{G}$. Here, we treated $\Sigma$ as a background field with no dynamics as is done in the literature $[23,31]$. As we will see, treating the Higgs as a background field is a harsh 
simplification and diminishes the composite phenomenology significantly. However, the way it is here, such construction is sufficient for the HP since we will compute the Higgs potential by fixing external legs at zero external momentum.

In the Coleman-Weinberg calculation, we perform the ressumation of all '-loop irreducible contributions of the Higgs external legs. By this approach, we can extract what the Higgs mass is and see how light it can be compared to $f$. Thoughout this section we will outline the calculation of [30]. Writing (2.62) in the unitary gauge leads to:

$$
\begin{aligned}
\mathcal{L}_{\text {eff }}^{\mathcal{G}}= & \frac{1}{2} \mathcal{P}^{\mu \nu}\left[\left(\Pi_{0}(p)+\frac{S_{h}^{2}}{4} \Pi_{1}(p)\right) B_{\mu} B_{\nu}\right. \\
& \left.+\left(\Pi_{0}(p)+\frac{S_{h}^{2}}{4} \Pi_{1}(p)\right) A_{\mu}^{a} A_{\nu}^{a}-2\left(\frac{S_{h}^{2}}{4} \Pi_{1}(p)\right) A_{\mu}^{3} B_{\nu}\right] \\
& \overline{q_{L}} \not p\left(\Pi_{0}^{L}(p)+\Pi_{1}^{L}(p) \frac{S_{h}^{2}}{2}\right) q_{L}+\overline{t_{R}} \not p\left(\Pi_{0}^{R}(p)+\Pi_{1}^{R}(p) C_{h}^{2}\right) t_{R}+ \\
& +\overline{t_{R}}\left(M_{1}(p) \frac{S_{h} C_{h}}{\sqrt{2}}\right) q_{L}
\end{aligned}
$$

From these expressions, we can extract the Feynman rules for such a theory.

$$
=\mathcal{P}_{\mathcal{T}}{ }^{\mu \nu} \frac{i}{\Pi_{0}\left(p^{2}\right)}
$$

The potential is obtained by summing all the 1PI contributions of closed gauge boson and fermion loops with the all external $\Sigma$ lines at zero momentum, as indicated by figures 2.7-2.8. This justifies the background filed assumption below. 


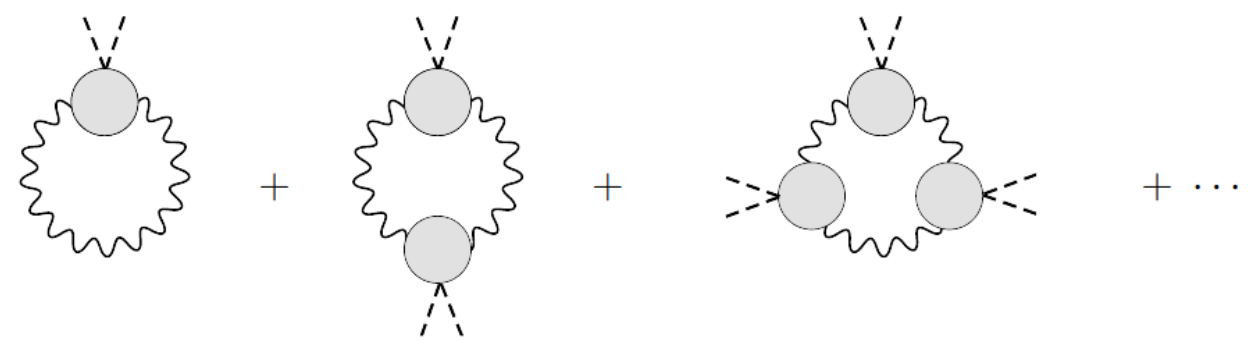

Figure 2.7: Coleman Weinberg calculation for the MCHM5 with form factors. Here are displayed the gauge boson loop contributions. The 1-loop ressumation is done with the external Higgses in zero momentum. This feature means that treating $\Sigma$ as a background field is acceptable here.

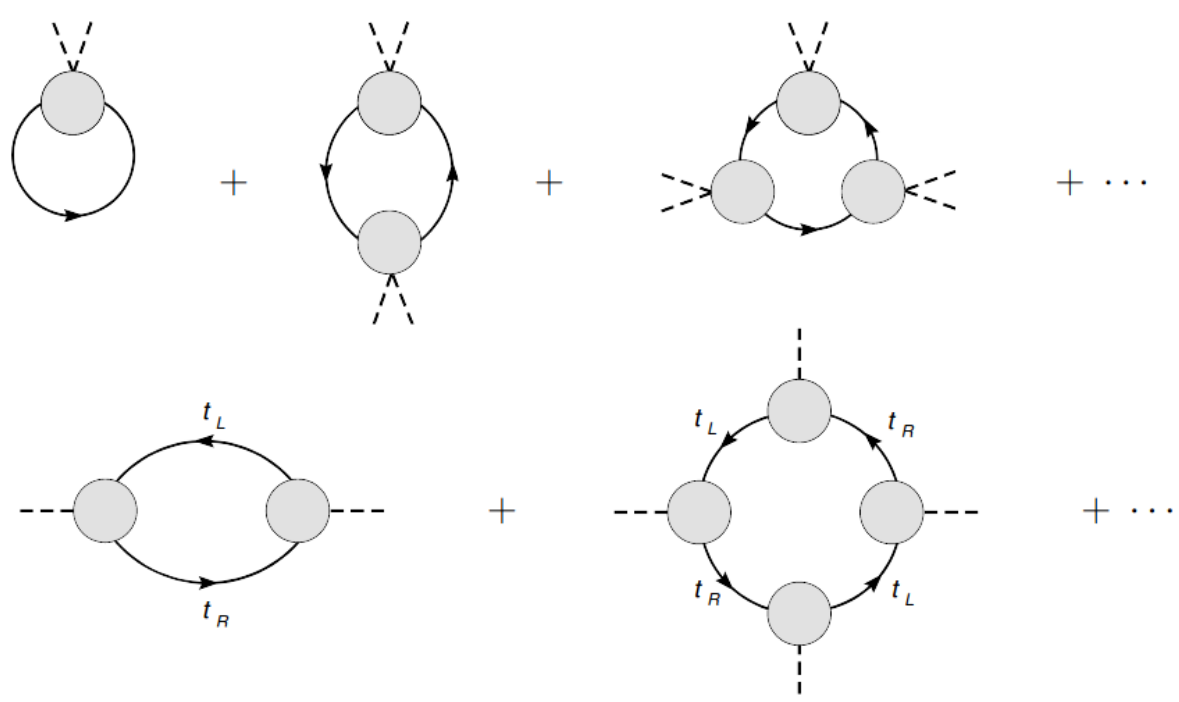

Figure 2.8: Coleman Weinberg calculation for the MCHM5 with form factors. Here are displayed the fermion 1-loop contributions.

The actual computation is not our primal concearn here, and we refer to [30, 73, 74] for further details. After ressuming, the gauge boson and fermion contributions 
are

$$
\begin{aligned}
V_{\text {eff }}^{\text {gauge }}(h)=\frac{9}{2} \int \frac{d^{4} q}{(2 \pi)^{4}} \log \left(1+\frac{\Pi_{1}\left(q^{2}\right)}{4 \Pi_{0}\left(q^{2}\right)} \sin ^{2}(h / f)\right) \\
V_{e f f}^{\text {fermion }}(h)=-2 N_{c} \int \frac{d^{4} q}{(2 \pi)^{4}} 2\left\{\log \left(1+\frac{\Pi_{1}^{L}\left(q^{2}\right)}{4 \Pi_{0}^{L}\left(q^{2}\right)} \cos (h / f)\right)\right. \\
+\log \left(1-\frac{\Pi_{1}^{R}\left(q^{2}\right)}{4 \Pi_{0}^{R}\left(q^{2}\right)} \cos (h / f)\right) \\
\left.+\log \left(1-\frac{\left(M_{1} \sin (h / f)\right)^{2}}{q^{2}\left(\Pi_{0}^{L}+\Pi_{1}^{L} \sin (h / f) / 2\right)\left(\Pi_{0}^{R}+\Pi_{1}^{R} \cos (h / f)\right)}\right)\right\}
\end{aligned}
$$

The finiteness of the potential is guaranteed by the convergence properties of form factors. Imposing constraints on their momentum behaviour ${ }^{6}$, the potential can be approximated by expanding the logariths at first order. Schematically it assumes the following form:

$$
V(h)=\alpha \cos \left(\frac{h}{f}\right)+\beta \sin ^{2}\left(\frac{h}{f}\right)
$$

where $\alpha$ and $\beta$ are functions of the form factors ${ }^{7}$.

The generation of EWSB then is given by the minimization of such potential. We can see that, to trigger EWSB one must have $\alpha \leq 2 \beta$. The minimum of the potential is at

$$
\xi=\frac{v^{2}}{f^{2}}=1-\left(\frac{\alpha}{2 \beta}\right)^{2}
$$

Once we define a model that has specific form factors, the fine-tuning question shifts from adjusting the parameters to actually calculating the expected Higgs mass. There will generally be a tuning of order $\xi$, which is an expected feature of CHMs. The calculation of the Coleman-Weinberg potential highlights how CHMs addresses the HP, recovering a natural theory. However, we are interested in the modifications of the Higgs dynamics due to compositeness, and for that, we must go further.

\footnotetext{
${ }^{6}$ Such as sum rules that we will explore in chapter 3

${ }^{7}$ Again, check [30] for explicit expressions.
} 


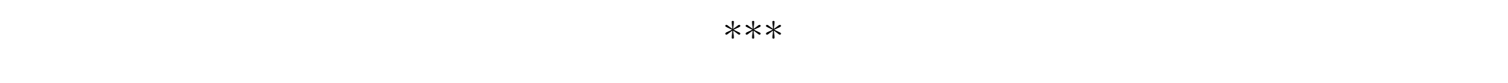

We dedicated this chapter to an inquiring review of pNGB CHMs. This has led to the main features that CHM lacks in describing composite phenomenology a full inclusion of momentum dependence in dynamical Higgs interactions.

Now we must obtain a formal and more complete picture of form factors. What remains is to understand the analytic structure of these functions and how they can be explicitly written. Then, we go to define the complete form factor theory of a CHM. In it, we shall restore the fully dynamical $\Sigma$, which was so far treated as a background field.

Working with an explicit realization of compositeness through the CHM shall enlighten us about the full consequences of a composite Higgs later on. 


\section{Chapter 3}

\section{Strong Dynamics via Nonpertubative methods}

The search for compositeness signals has led us to consider the full extent of momentum dependence in interactions. Now, the first step is to decipher the BSM effects by going into a limit of high momentum transfer in collider experiments.

These momentum dependent effects of the new composite sector shall be expressed through the analytical properties of form factors. So, in order to construct their expressions, we must look at the general properties of point functions in any interacting theory, regardless of their interaction strength.

Even though we have limited access to the nonperturbative regime of QFT's, there are some tools to classify their general behaviour. A good lead is by looking into the spectrum of the theory, where we shall find a way of predicting sensible effects into the two-point propagators. Such is done through the spectral decomposition and will define the general shape of the form factors later on.

It is assumed that a strong sector predicts composite bound states. We want to know how this behaviour emerges after confinement, moulding these resonances. The $1 / N$ expansion is a general classification that provides a grounding for this class of Higgs phenomenology. Unintentionally, using it makes model-building very QCD-like. 
After this part on generalities, it is necessary to use tools and approximations to constraint the behaviour of form factors. These tools will aim to get useful expressions for our formulation. Imposing constraints to form factors might be a very productive way forward in exploring this regime. Some fundamental properties of field theories like unitarity, analyticity and the restoration of $\mathcal{G}$ symmetry at high energies are greatly valuable in our context.

All of these tools had their role for dealing with QCD in the nonperturbative regime. Because of it, they are necessarily driven towards the specific problem of a hadronic theory and have their limitations for Higgs applications. Alternatively, one could ask if this QCD-like behaviour is present in all strongly interacting QFT's, and what is the full range of possibilities for the sector that is responsible for confining the Higgs.

Most importantly, the tone here is to explore a concrete implementation of momentum dependence when there is a composite sector lying ahead at the $\mathrm{TeV}$ range. Of course, there are many possibilities for the strong sector that confines the Higgs; the one we present is just one of them.

\subsection{Spectral Properties}

Built in the conceptual framework of QFT is the artificial division of the free and interacting parts of the dynamics. In such division, we include the twopoint interactions into the free part and the higher point ones into the perturbation lagrangian. Such division is done purely from a calculatory standpoint and has not any particular physical significance.

However, when dealing with quantum corrections, the parameters of the theory get shifted and acquire a running dependence with energy. This phenomenon redefines the artificial divisions made at the beginning - the theory regulates itself, and this change of parameters often can be explicitly calculated in the perturbative regime. 
The starting free particles form part of the spectrum, but their point interactions change due to the quantum nature of the theory. Loops of intermediate multi-particle states modify the bare parameters. In particular, the weakly coupled regime implicitly assume that radiative corrections only modify the behaviour of the theory minorly, making perturbative assumptions predictive.

However, the interaction effects can be more dramatic than that. It might occur the formation of bound-states in strongly interacting systems, a phenomenon that is nonperturbative by its nature. In confining theories such as QCD, the formation of bound-states is a central aspect of the theory itself, even though this process is not yet completely understood. Since this is a hard problem, we will just assume the existence of resonances as single-particle contributions. Later we shall ask for a more profound prescription.

At this point we can define the spectrum as the vacumm with and the single or multiparticle eingenstates of the operator $P^{2}$, each with its mass eingenvalue. Their structure is shown in figure 3.1.The single particle states are the free elementary plus the possible bound-states generated dynamically. The multiparticle states are defined by the continuum formed by the fock-states with more than one particle in it.

\section{General Spectrum of a QFT}

$|0\rangle$ : vacuum state $E=0$

$|1, k\rangle: 1$ particle states $k^{2}=m^{2}$.

$|2, k\rangle$ : bound states $k^{\prime 2}=m^{\prime 2}$.

$|\lambda, k\rangle$ : multi particle particle states with $\lambda$ as a continuous index.

A more sensible way to define the physical spectrum of a theory is related directly to the properties of the S-matrix [84]. This contours the artificial divisions made in the perturbative regime. The S-matrix is an analytical function of the external momentum of asymptotic states. Non-analyticities like poles and branch 


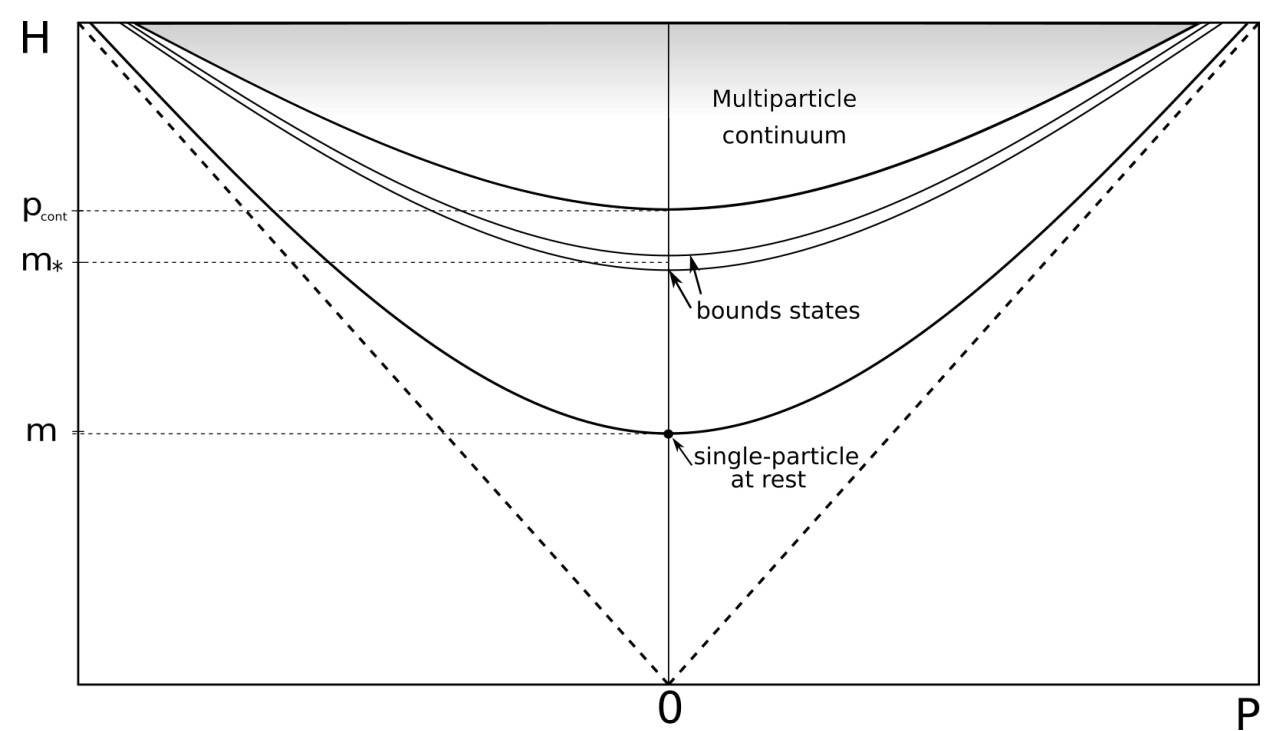

Figure 3.1: Eingenstates of the $P^{\mu}=(H, P)$ operator. Figure based on [12]. The eingenstates of the momentum operator defines the particles masses. Their structure can be devided in single-elementary ,single-bound-state or a multiparticle one.

cuts always indicates physical processes taking place, and provide a well-defined description of particles states.

We can define a function that has the spectral properties desired for our theory.

$$
f(p) \equiv \sum_{\alpha}|\langle 0|\phi(0)| \alpha\rangle|^{2} \delta^{4}\left(p-P_{\alpha}\right)
$$

where $\alpha$ is the index of the Hilbert space complete basis, with the states being eingenvectors of the $P^{\mu}$ operator. This function has support only for the single particle mass hyperboloid $p^{2}=m^{2}$ and the multi-particle continuum. Also, $f(p)$ is a Lorentz scalar function so it only depends on $p^{2}$ and vanishes for $p_{0}<0$. Since $|\lambda\rangle$ are physical states they must be on-shell, so we have also $p_{0} \geq 0$ and $p^{2}>0$. All of these allow us to define the spectral function $\rho\left(p^{2}\right)$, which is positive and with support only on the physical spectrum.

$$
\frac{1}{(2 \pi)^{3}} \theta\left(p_{0}\right) \rho\left(p^{2}\right)=\sum_{\alpha}|\langle 0|\phi(0)| \alpha\rangle|^{2} \delta^{4}\left(p-P_{\alpha}\right)
$$




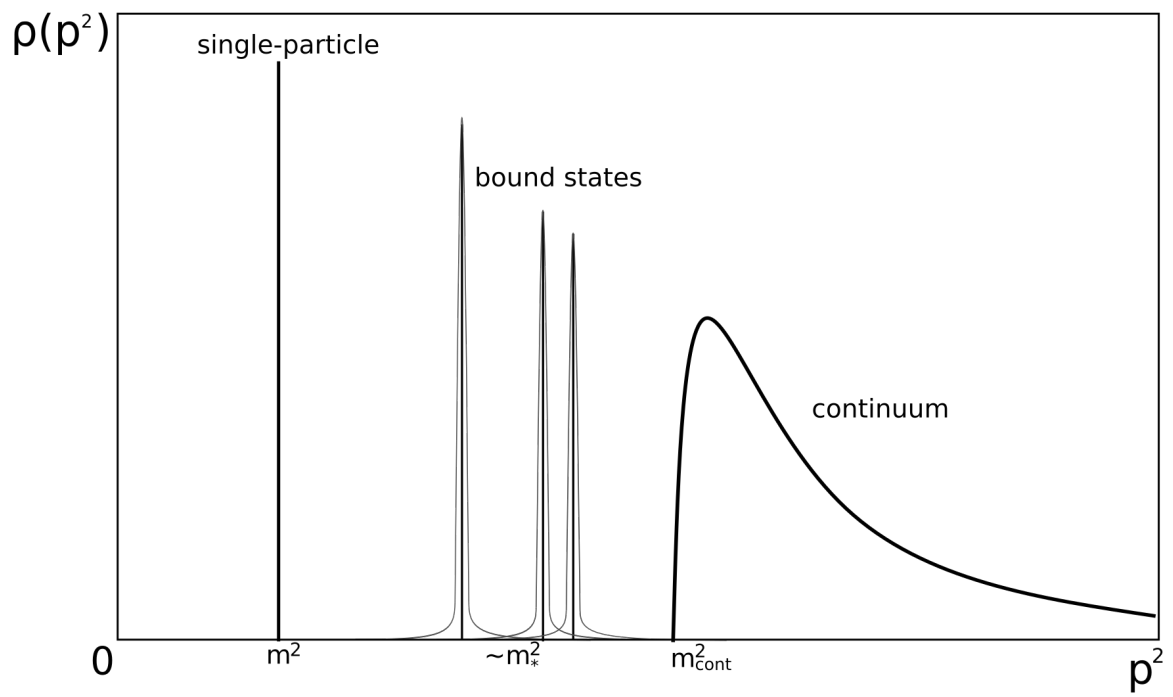

FIgURE 3.2: General spectral density structure of an interacting quantum field theory.

The matrix element for the single-particle states is defined up to a normalization factor $Z$ as the plane wave element

$$
\langle 0|\phi(0)| k\rangle_{\text {in }}=\frac{Z^{1 / 2}}{(2 \pi)^{3 / 2} \sqrt{2 E(k)}} e^{i k \cdot x} .
$$

Up to a normalization factor, we can determine the spectral function for the stable 1-particle states.

$$
\begin{array}{r}
\frac{1}{(2 \pi)^{3}} \theta\left(p_{0}\right) \rho_{1 \text { part }}\left(p^{2}\right)=\int d^{3} k|\langle 0|\phi(0)| k\rangle|^{2} \delta^{4}(p-k) \\
=\frac{Z}{(2 \pi)^{3} 2 E(P)} \delta\left(p_{0}-E(p)\right) \theta\left(p_{0}\right) \\
\rho_{1 \text { part }}\left(p^{2}\right)=Z \delta\left(p_{0}-E(p)\right) \delta\left(p_{0}+E(p)\right)=Z \delta\left(p^{2}-m^{2}\right)
\end{array}
$$

The spectral function tells us all about the intermediate physical states of the theory. For interacting theories, it will generally have singularities at renormalized masses of one-particle states and bound states, and a branch cut for multi-particle states. The analyticity of the spectral function and the S-matrix are correspondent in this manner. 
Each pole corresponds to a single particle state, with the location of the pole being the physical mass of the particle. The branch cuts signal the presence of a continuum of states, such as the one indicated by the threshold of multi-particle production. The general spectral function is pictured in figure 3.2

Relation (3.5) is valid for stable particles that can always be asymptotic states. In the case of unstable resonances, where the particle can decay into lighter ones, the dispersion relation $p^{2}=m^{2}$ becomes imaginary. In such a case, the spectral function is not a delta function anymore and has a continuous $p^{2}$ dependence that is controlled by the decay widths. Physically, their poles are getting deeper into the analytic plane, acquiring a larger imaginary contribution $[18,19]$.

To see that, we can calculate the complex contributions to the pole of the propagator, that defines $p^{2}=m^{2}$. Defining $-i M^{2}\left(p^{2}\right)$ as the self-energy, which is the sum of the one particle-irreducible (1PI) insertions into the propagator, we can obtain the exact two-point function. ${ }^{1}$

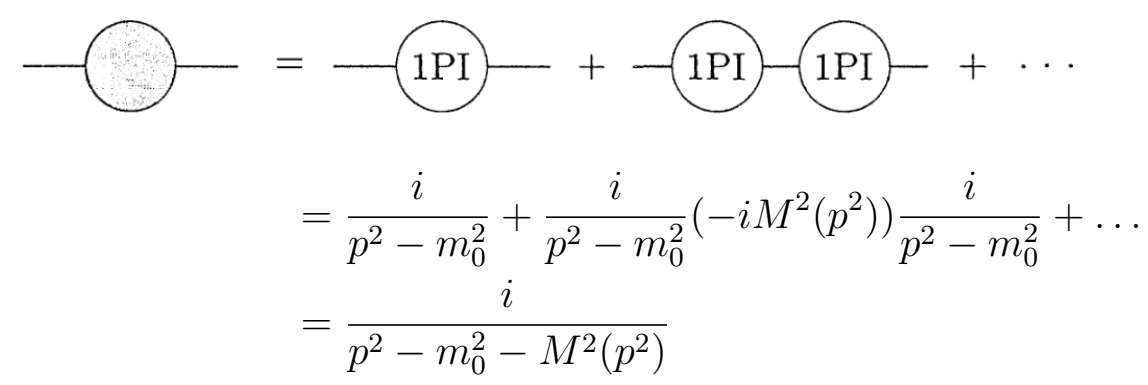

where we defined $m_{0}$ as the bare mass to differenciate with the physical mass $m$.

When the particle is stable, unitarity guarantees that $M^{2}\left(p^{2}\right)$ is real. In this case, the physical mass is defined by the pole in the propagator at $p^{2}=m^{2}$, that is $m^{2}=m_{0}^{2}+M^{2}\left(m^{2}\right)$. The pole lies in the real $p^{2}$ axis and gives a real delta function contribution in the spectral function.

Now, in the case of unstable particles $M^{2}\left(p^{2}\right)$ aquires an imaginary part, and the physical mass must be defined as $m^{2}=m_{0}^{2}+R e\left[M^{2}\left(m^{2}\right)\right]$. The pole is not

\footnotetext{
${ }^{1}$ Figure taken from [73].
} 
anymore in the real axis, because of the remaining imaginary part of $M^{2}\left(p^{2}\right)$.

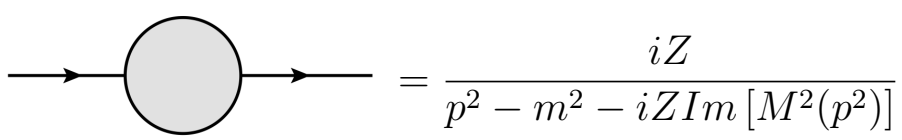

The propagator modifications due to the presence of self-interactions suggest a link between the spectral properties and the general form of the two-point-function. We shall turn to derive what this relation is through the spectral representation.

\subsubsection{The Spectral Decomposition}

Now we desire a connection of the spectrum of the theory and the general form of the two-point function [61]. When computing the exact propagator, we realized that the analytic form of the two-point function could encapsulate effects from the interactions through the self-energies. An explicit connection with the more general spectral function is needed.

Starting with the two point correlator between two scalar fields, it can be decomposed as following.

$$
\begin{aligned}
\langle\phi(x) \phi(y)\rangle & =\sum_{\lambda} \int \frac{d^{3} p}{(2 \pi)^{3}} \frac{1}{2 E_{\vec{p}}(\lambda)}\left\langle\Omega|\phi(x)| \lambda_{p}\right\rangle\left\langle\lambda_{p}|\phi(y)| \Omega\right\rangle \\
& =\sum_{\lambda} \int d \Pi_{\lambda} e^{-i p_{\lambda}(x-y)}\left|\left\langle\Omega|\phi(0)| \lambda_{0}\right\rangle\right|^{2} \\
& =\int \frac{d^{4} p}{(2 \pi)^{4}} e^{-i p(x-y)}\left\{\sum_{\lambda} \int d \Pi_{\lambda} \delta^{4}\left(p-p_{\lambda}\right)\left|\left\langle\Omega|\phi(0)| \lambda_{0}\right\rangle\right|^{2}\right\}
\end{aligned}
$$

The quantity in brackets is the spectral function defined in (3.2). Now, we can write the correlator in terms of the propagator $\Delta_{+}\left(x-y ; m^{2}\right)$.

$$
\begin{aligned}
\Delta_{+}\left(x-y ; m^{2}\right) & =\int \frac{d^{3} p}{(2 \pi)^{3}} \frac{1}{2 \sqrt{\vec{p}^{2}-m^{2}}} e^{-i p(x-y)} \\
& =\int \frac{d^{4} p}{(2 \pi)^{4}} e^{-i p(x-y)} \theta\left(p^{0}\right) \delta\left(p^{2}-m^{2}\right)
\end{aligned}
$$




$$
\langle\phi(x) \phi(y)\rangle=\int_{0}^{\infty} d m^{2} \rho\left(m^{2}\right) \Delta_{+}\left(x, y ; m^{2}\right)
$$

The Feynman propagator can be used to obtain the time ordered version of the two-point function.

$$
i \Delta_{F}\left(x-y ; m^{2}\right)=\theta\left(x^{0}-y^{0}\right) \Delta_{+}\left(x-y, m^{2}\right)-\theta\left(y^{0}-x^{0}\right) \Delta_{+}\left(y-x ; m^{2}\right)
$$

Then,

$$
\langle\mathcal{T} \phi(x) \phi(y)\rangle=i \int \frac{d^{4} p}{(2 \pi)^{4}} e^{i p(x-y)} \Pi\left(p^{2}\right)
$$

With,

\section{Spectral Decomposition}

$$
\Pi\left(p^{2}\right)=\int_{0}^{\infty} d m^{2} \frac{\rho\left(m^{2}\right)}{p^{2}-m^{2}}
$$

The two-point function is an analytical function of external momentum, meaning its poles and branch cuts define the entire function. From the other point, the spectral function gives the physical content of the theory, with all the possible states. The usefulness of the spectral decomposition is to link the analytical properties and the physical content of the theory. This relationship will be the leading guide in constructing the form factors. The analytical properties are pictured in figure 3.3.

By using the general form of the spectral funtion for the single particle state (3.5) we can factor out the lowest exitation that corresponds to the free particle Lagrangian, assuming it is stable. By substituting (3.5) into (3.18) we get

$$
\Pi\left(p^{2}\right)=\frac{i Z}{p^{2}-m^{2}}+\int_{m_{*}}^{\infty} d \mu^{2} \frac{\rho\left(\mu^{2}\right)}{p^{2}-\mu^{2}}
$$

One can see that a confining theory require the wavefunction normalization to be zero, $Z=0$. This requirement happens because the confined elementary constituents are not asymptotic states, nor they are produced as resonant excitations.

Also, if the strongly interacting system produces a spectrum of single particle 


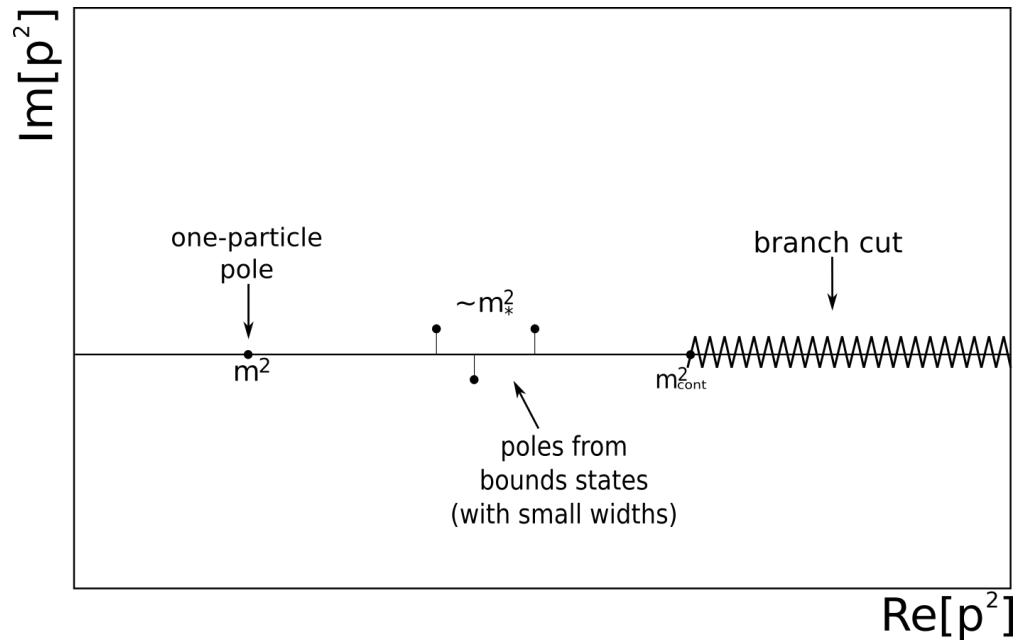

FiguRE 3.3: Analytic structure of a general two point function. There are poles for single-particle states (Assumed to be stable), poles of bound-states with small widths, and a branch-cut for multiparticle states. Note that the non-analycities of the spectral function correspond to physical processes of interest.

bound-states, they should be accessible through the spectral function. We can divide $\rho\left(p^{2}\right)$ into a single particle bound-state (BS) contribution and a multiparticle one (MS).

$$
\Pi\left(p^{2}\right)=\int_{m_{*}}^{\infty} d \mu^{2} \frac{\rho_{B S}\left(\mu^{2}\right)}{p^{2}-\mu^{2}}+\int_{4 m^{2}}^{\infty} d \mu^{2} \frac{\rho_{M S}\left(\mu^{2}\right)}{p^{2}-\mu^{2}}
$$

Isolating one of these resonances, we can translate the bound-state expression for the spectral decomposition into the exact propagator form.

$$
\int_{m_{*}-\Delta m_{*}}^{m_{*}+\Delta m_{*}} \frac{\rho_{B S}\left(\mu^{2}\right)}{p^{2}-\mu^{2}} d \mu^{2} \sim \frac{i Z}{p^{2}-m_{*}^{2}-i Z \operatorname{Im}\left[M^{2}\left(p^{2}\right)\right]}
$$

where, the interval $\left(m_{*}-\Delta m_{*}, m_{*}+\Delta m_{*}\right)$ indicates the vicinity of the bound-state contribution.

This relation suggests that if $\operatorname{Im}\left[M^{2}\left(m^{2}\right)\right]$ is small, the spectral form can be further simplified. This effect happens because the distribution becomes concentrated at the pole mass $m^{2}$. The natural question here is, do we expect these stable resonances in such a strongly interacting system?

To address this question, we can use a systematic approach to characterize the 
hadron-like states of a theory such as QCD. Such characterization is done through a perturbative expansion proposed by 't Hooft [57, 60].

\subsection{The 1/N Expansion}

The $1 / N$ expansion is a perturbative scheme for a $S U(N)$ gauge theory in terms of the number of colours N. In a strongly interacting system, perturbation theory in terms of the coupling constant is not well controlled and does not provide much insight into the full theory. Here, the strategy is to recover a perturbative scheme in terms of the external parameter $N$, defining the theory in the large- $\mathrm{N}$ limit.

't Hooft showed that as $N \rightarrow \infty$ the theory simplifies so that mesons and glue states are free, stable, and non-interacting. Also, the meson widths are of order $1 / \sqrt{N}$, and meson-meson elastic scattering amplitudes get suppressed to order $1 / N$.

Of course, in QCD the gauge group with $N=3$ does not seems to provide a perfect approximation, but the expansion is qualitatively useful. It organizes the hadronic spectrum and provides arguments for the seemingly free and stable behaviour or resonances. Even though this technique still uses some perturbative analysis in terms of the coupling constant, there are certain classes of diagrams that share the same features at all orders in the perturbative expansion. The assumption is that after the ressumation, this set of diagrams is representative of the final theory. ${ }^{2}$

These diagrams are called planar and are better defined through the 't Hooft double-line notation for representing $S U(N)$ colour diagrams, as depicted in figure 3.4 .

In this QCD-like theory, there are $N$ colours of quarks and $N^{2}-1 \approx N^{2}$ gluons. As an example, the vacuum polarization contributions to the gluons twopoint function are pictured in figure 3.5.

\footnotetext{
${ }^{2}$ The final class of diagrams is also assumed to generate the confinement phenomenon.
} 

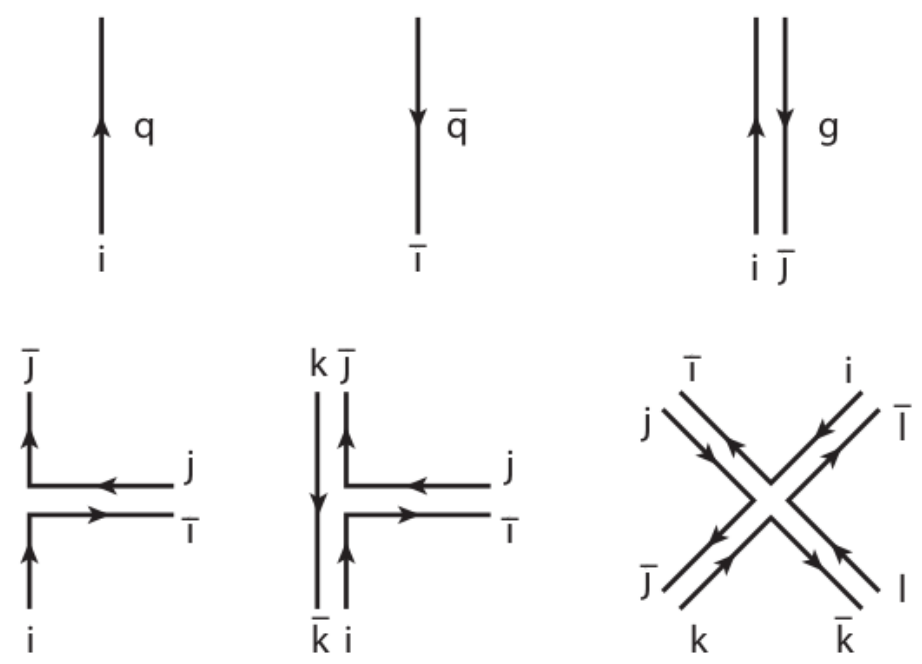

Figure 3.4: 't Hooft double line notation. A quark or anti-quark are represented by one line, as they carry just one color label. The gluons have two lines associated with them
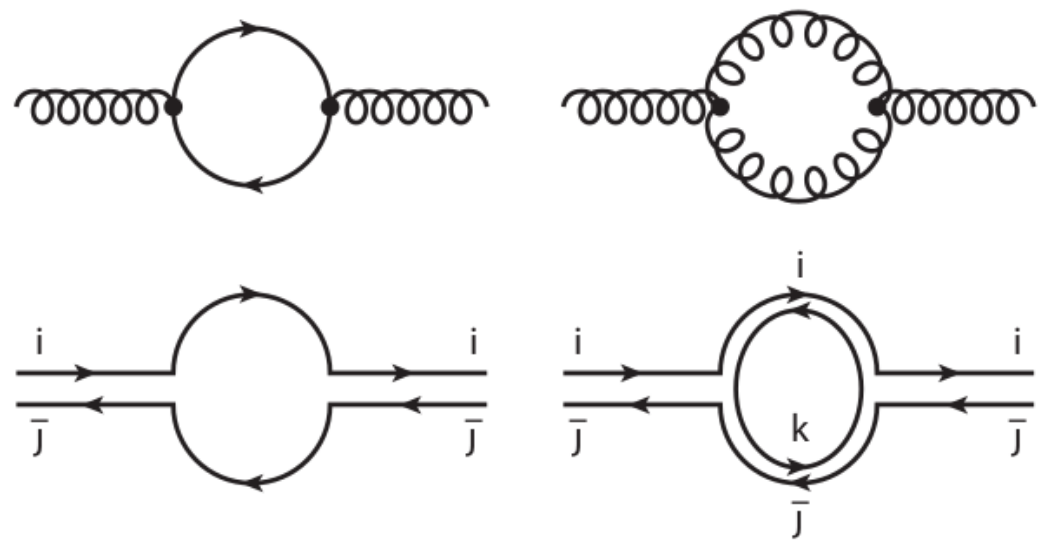

FigURE 3.5: Vacuum polarization of the glouns two-point function.

Inside the gluon loop there is a colour index line $k$ that is free to take the values $k=1, \ldots, N$. Correspondingly, this diagram is associated with a multiplicative factor $N$ and dominates over the quark loops as $N \rightarrow \infty$. However, the perturbation is ill-defined if we wish to choose the factor $N$ as expansion parameter since the contributions diverge. To contour this issue, we rescale the coupling constant to be $g / \sqrt{N}$, with fixed $g$ and $N \rightarrow \infty$. The loop factor then goes to a well-behaved limit.

$$
\left(\frac{g}{\sqrt{N}}\right)^{2} \cdot N \rightarrow g^{2}
$$


With this definition, the disposal of quark loop diagrams is explicit. After the coupling constant rescaling they go like

$$
\left(\frac{g}{\sqrt{N}}\right)^{2} \cdot 1 \sim \frac{g^{2}}{N} \rightarrow 0
$$

and each internal quark loop has this suppression factor of $1 / N$.

The Higher loops can be trated with the same methodology. Consider the two loop contribution of figure 3.6.
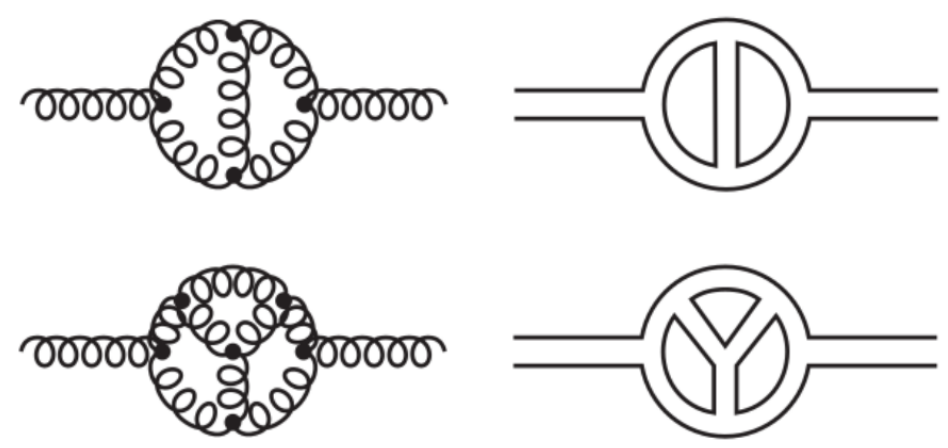

FigURE 3.6: Higher loops for the vacuum polarization of the gluons.

Such diagrmas go like:

$$
\left(\frac{g}{\sqrt{N}}\right)^{4} \cdot N^{2} \rightarrow g^{4}
$$

Similarly the three loop goes like

$$
\left(\frac{g}{\sqrt{N}}\right)^{6} \cdot N^{3} \rightarrow g^{6}
$$

All of these cases belong to the class of planar diagrams, that do not self intersect at some internal point. For non-planar diagrams, the combinatorial analysis favours their disposal. For example, consider yet another loop in figure 3.7.

Since there is only one internal running index, this diagram scale as

$$
\left(\frac{g}{\sqrt{N}}\right)^{6} \cdot N \rightarrow \frac{g^{6}}{N^{2}}
$$



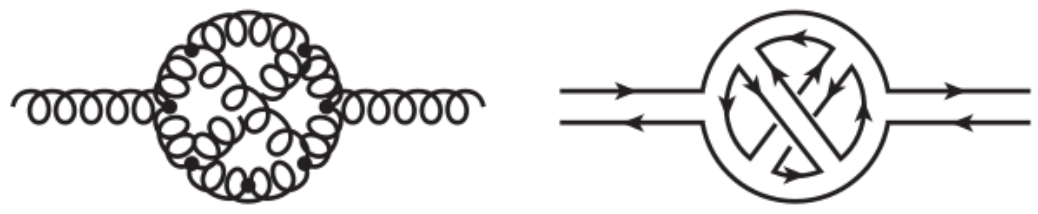

FiguRE 3.7: Example of a non-planar diagram in the gluon two-point function. Note that there is only one running index.

therefore, it is discarted as $N$ approaches infinity. As a general result, all non-planar graphs are suppressed by a factor of $1 / N^{2}$ and can be desconsidered in the large- $N$ limit [57, 60, 75].

Even though the evaluation of all the planar diagrams is a hard task, the conclusions are fairly simple. They provide some insight into the qualitative features of a strongly interacting theory in the large- $\mathrm{N}$ limit.

- The meson-like particles are free, stable and non-interacting.

- Meson-like particle decays are proportional to $1 / \sqrt{N}$, so they are narrow states.

- Meson-meson scattering amplitudes get suppressed to $1 / N$.

- Mesons are pure quark-like $(q \bar{q})$ states, with no quark-antiquark sea.

- The exotic meson-meson bound states are forbidden.

- Infinite tower of glueball states, with widths $1 / N^{2}$.

These consequences are important qualitative guidelines for modelling the composite sector of the Higgs. We can use this QCD-driven approach to firm a concrete realization of the strong sector. However, there could be other behaviours that depart from this general picture.

Contrary to the Large-N, in QCD, we have the $\sigma$ and the $\rho$, which are broad and strongly interacting at the hadron level. Also, there are all sorts of meson-meson systems and exotic bounds states like glueballs and pentaquarks. Additionally, sending $N \rightarrow \infty$ do not describe the qualitative behaviour of baryons as it was supposed 
to do. All of these corroborate to go beyond such limit. However, we postpone such discussion to chapter 6 .

\subsection{Tools for Form Factor Model-Building}

Assuming the spectral properties and analytical structure of the previous sections and the Large- $\mathrm{N}$ limit, extracting practical expressions from the spectral decomposition is a more attainable task now.

The form factors will inherit the analytic properties of the point-functions. Their construction is based on integrating-out the massive resonances while keeping its dynamical effects. Even though these massive states will not be explicitly written, their influence will still be on the generation of the dynamical couplings of the theory. Ultimately, they will be accessible through the momentum transfer of Higgs processes.

For the task of writing the expressions, we will present some tools that were amply used in the QCD context and will be of use here. The objective is to reduce the integral form of the spectral function into an explicit function of the external momenta.

\subsubsection{Narrow Resonances Limit}

Previously, we argued that the spectral decomposition for bound states is related to the exact propagator as

$$
\int_{m_{*}-\Delta m_{*}}^{m_{*}+\Delta m_{*}} \frac{\rho_{B S}\left(\mu^{2}\right)}{p^{2}-\mu^{2}} d \mu^{2}=\frac{i Z}{p^{2}-m_{*}^{2}-i Z \operatorname{Im}\left[M^{2}\left(p^{2}\right)\right]}
$$

One of the consequences of the large- $\mathrm{N}$ limit is that the resonance widths are

narrow in the composite theory. In this case, the $\operatorname{Im}\left[M^{2}\left(m^{2}\right)\right]$ is small compared to 
the particle pole mass. Therefore we can approximate the width as:

$$
\Gamma=-\frac{Z}{m} \operatorname{Im}\left[M^{2}\left(m^{2}\right)\right]
$$

Then, the spectral decomposition can be factored to all single particle states when resonances are narrow.

$$
\begin{aligned}
\Pi\left(p^{2}\right) & =i \int d^{4} x e^{i p(x-y)}\langle\mathcal{T} \phi(x) \phi(y)\rangle \\
& =\frac{i Z}{p^{2}-m^{2}}+\sum_{i=1}^{N_{S}} \frac{i F_{i}^{2}}{p^{2}-m_{i}^{2}+i m_{i} \Gamma_{i}}+\int_{\sim 4 m^{2}}^{\infty} d \mu^{2} \frac{\rho\left(\mu^{2}\right)}{p^{2}-\mu^{2}}
\end{aligned}
$$

where the constants $F_{i}=\left|\left\langle\lambda_{0}\left|\phi_{0}\right| \Omega\right\rangle\right|^{2}$ are taken as a phenomenological input to the theory, and $N_{S}$ is the number of narrow scalar resonances in such theory.

The generalization to fermions and gauge bosons comes with the replacement of the scalar propagator to the correspondent propagator for each spin particle.

$$
\begin{aligned}
\Pi_{F}\left(p^{2}\right)=i \int d^{4} x e^{i p(x-y)}\langle\mathcal{T} \overline{\psi(x)} \psi(y)\rangle \\
=\frac{i Z}{\not p-m}+\sum_{i=1}^{N_{F}} \frac{i F_{i}(\not p-m)}{p^{2}-m_{i}^{2}+i m_{i} \Gamma_{i}}+\int_{\sim 4 m^{2}}^{\infty} d \mu^{2} \frac{\rho\left(\mu^{2}\right)}{\not p-\mu} \\
\Pi_{V}^{\mu \nu}\left(p^{2}\right)=i \int d^{4} x e^{i p(x-y)}\left\langle\mathcal{T} A^{\mu}(x) A^{\nu}(y)\right\rangle \\
=\frac{i Z\left(\eta^{\mu \nu}-\frac{p^{\mu} p^{\nu}}{p^{2}}\right)}{p^{2}-m^{2}}+\sum_{i=1}^{N_{V}} \frac{i F_{i}^{2}\left(\eta^{\mu \nu}-\frac{p^{\mu} p^{\nu}}{p^{2}}\right)}{p^{2}-m_{i}^{2}+i m_{i} \Gamma_{i}}+ \\
+\int_{\sim 4 m^{2}}^{\infty} d \mu^{2} \rho\left(\mu^{2}\right) \frac{\left(\eta^{\mu \nu}-\frac{p^{\mu} p^{\nu}}{p^{2}}\right)}{p^{2}-\mu^{2}}
\end{aligned}
$$

The treatment of the continuum part is more involved since it depends on specific details of the intermediate virtual state corrections. This treatment is no trivial task to be done non-perturbatively. In general, the threshold for multi-particle production is at energies above the single-particle pole region (e.g. $\sim 4 m^{2}$ ), and the 
first-order effects should come from the single-particles states alone.

With this in mind, we further assume that the continuum can be neglected in terms of only the poles of the spectral function.

The situation is an infinite tower of resonances, each signalling a pole on the spectral function. In this set of infinite particles, we can focus on the lowest mass states as they are closer to the threshold for experimental visibility. In the same manner, as we had discarded the higher energy effects of the continuum, we can try to simplify the analytic structure of the theory here. Further simplification is achieved by just considering a small number of lower-end bound-state excitations.

In $\mathrm{QCD}$, the pion form factor is well reproduced using this strategy. The $\rho$ resonance is the lowest state that shares the photon quantum numbers. Practically, the actual measurement of the form factor effect is well matched by an expression with a simple pole at the mass of $770 \mathrm{MeV}$ of the $\rho$.

The two-point function assumes a simple expression with only these lower-end part of the resonant spectrum.

$$
\Pi\left(p^{2}\right) \approx \frac{i Z}{p^{2}-m^{2}}+\frac{i F^{2}}{p^{2}-M^{2}+i M \Gamma}
$$

\subsubsection{Weinberg Sum Rules}

In the previous section, we constrained the expressions from the lower end of the spectrum. This was done in order to reach a simple form for the two-point function. Also, it is possible to include constraints from the expected UV properties of the theory. These are generally implemented through sum rules $[52,51]$.

The Weinberg sum rules are a set of relations that applies when dealing with spectral functions of a spontaneous breaking to a subgroup $\mathcal{H}$. The sum rules guarantee the $\mathcal{G}$-symmetry restoration at high energies, that is a general property of any theory with a composite Higgs.

First, we assign two contributions to the two point-functions. $\Pi_{a}$ is the assigned to the unbroken correlator and $\Pi_{\hat{a}}$ to the broken one. The sum rule's objective 
is to enforce that at the UV, the momentum behaviour of the form factors is the same.

$$
\lim _{|p| \rightarrow \infty}\left(\Pi_{a}^{\mu \nu}(p)-\Pi_{\hat{a}}^{\mu \nu}(p)\right)=0
$$

The Weinberg sum rules are obtained by requiring the asymptotic limit (3.33) to respect a spectral function decrease faster than $\frac{1}{p^{2}}$ and $\frac{1}{p^{4}}$ as $p \rightarrow \infty$.

$$
\begin{aligned}
& \lim _{|p| \rightarrow \infty} p^{2}\left(\Pi_{a}^{\mu \nu}(p)-\Pi_{\hat{a}}^{\mu \nu}(p)\right)=0 \\
& \lim _{|p| \rightarrow \infty} p^{4}\left(\Pi_{a}^{\mu \nu}(p)-\Pi_{\hat{a}}^{\mu \nu}(p)\right)=0
\end{aligned}
$$

We can decompose the ח's with the spectral representation. The convergence of the two-point function is assumed to hold order by order. So, expanding $1 /\left(p^{2}-\right.$ $\left.\mu^{2}\right) \approx 1 / p^{2}+\mu^{2} / p^{4}$ and imposing (3.34) - (3.35) will lead to Weinberg sum rules for spectral functions. Projecting the transverse part of the correlator, the sum rule (3.34) becomes

$$
\int_{0}^{\infty} d \mu^{2} \rho^{a}\left(\mu^{2}\right)=\int_{0}^{\infty} d \mu^{2} \rho^{\hat{a}}\left(\mu^{2}\right)+f_{\pi}^{2}
$$

where the appearance of the Goldstone matrix element $f_{\pi}$ is due to the broken current sensitivity to its goldstone exchange.

The second sum rule (3.35) for the spectral function is

$$
\int_{0}^{\infty} d \mu^{2} \rho^{a}\left(\mu^{2}\right) \mu^{2}=\int_{0}^{\infty} d \mu^{2} \rho^{\hat{a}}\left(\mu^{2}\right) \mu^{2}
$$

More explicitly, using the large-N limit with one vector meson dominance we get

$$
F_{\rho}^{2}-F_{a}^{2}-f_{\pi}^{2}=0
$$

where $F_{\rho}, F_{a}$ are the matrix elements for creating the corresponding resonant states associated with unbroken and broken currents. 
Also, the second sum rule becomes

$$
F_{\rho}^{2} m_{\rho}^{2}-F_{a}^{2} m_{a}^{2}=0
$$

Turning to fermionic contributions, it is instructive to recover the terminology of the MCHM5. There, we had form factors for a fourplet and singlet contributions. The sum rules analysis is the same as for the vectorial currents, and we can impose the sum rules to find:

$$
\begin{aligned}
& \lim _{|p| \rightarrow \infty} p^{2}\left(\Pi_{1}^{L}(p)-\Pi_{\mathbf{4}}^{L}(p)\right)=0 \\
& \lim _{|p| \rightarrow \infty} p^{2}\left(\Pi_{1}^{R}(p)-\Pi_{\mathbf{4}}^{R}(p)\right)=0
\end{aligned}
$$

Substituting the explicit forms we get to

$$
\begin{aligned}
& \left|y_{L 1}\right|^{2}-\left|y_{L 4}\right|^{2}=0 \\
& \left|y_{R 1}\right|^{2}-\left|y_{R 4}\right|^{2}=0
\end{aligned}
$$

where these constants are the mixing couplings of left and right-handed fermion multiplets.

The practical use of sum rules is to reduce internal parameter dependence. This reduction is crucial when studying the phase space of the theory for phenomenological signals. Nevertheless, the sum rules are a concrete prescription on the UV behaviour of the theory.

Another feature of the imposition of the sum rules is that the ColemanWeinberg potential of the theory will result to be finite $[96,28]$. This feature is expected in any CHM that claims to solve the hierarchy problem. Thus, imposing the sum rules is desired in model-building. 


\subsubsection{Form Factors}

Up until now, we have referred to the form factors and the correlation functions as the same. The distinction is a bit more subtle. The point functions include the entire dynamics of an interacting theory, including the propagation of the lowest excitation that we identify as the elementary degrees of freedom.

The form factors are a generic designation to the effects of integrating out the heavy states. These are not expected to appear explicitly at low energies. However, their contributions modify the dynamics of point functions and inherit the analytical behaviour of the theory.

Form factors are momentum dependent contributions that alter the elementary behaviour of interactions. They signal the presence of internal structure and can be used in the construction of effective lagrangians such as the MCHM5 lagrangian we presented earlier in chapter 2 .

$$
\begin{aligned}
\mathcal{L}_{\text {eff }}^{\mathcal{G}}= & \frac{1}{2} \mathcal{P}^{\mu \nu}\left(\Pi_{0}(p) \operatorname{Tr}\left(A_{\mu} A_{\nu}\right)+\Pi_{1}(p) \Sigma^{T} A_{\mu} A_{\nu} \Sigma\right)+ \\
& +\overline{Q_{L}} \not p\left(\Pi_{0}^{L}(p)+\Pi_{1}^{L}(p) \Sigma \Sigma^{T}\right) Q_{L}+ \\
& +\overline{T_{R}} \not p\left(\Pi_{0}^{R}(p)+\Pi_{1}^{R}(p) \Sigma^{T} \Sigma\right) T_{R}+ \\
& +\overline{Q_{L}}\left(M_{0}(p)+M_{1}(p) \Sigma \Sigma^{T}\right) T_{R}
\end{aligned}
$$

For each conserved current, we identify a form factor that represents a resonance exchange according to the spectral decomposition. These form factors correspond to self-energy contributions to the elementary fields two-point functions.

For example, an fourplet fermionic quadratic lagrangian has the general form:

$$
\begin{array}{r}
\mathcal{L}=\bar{Q}_{L 4} \not p Q_{L 4}+\Pi_{4}^{L}\left(p^{2}\right) \bar{Q}_{L 4} \not p Q_{L 4} \\
\Pi_{4}^{L}\left(p^{2}\right) \equiv \frac{f_{4}^{L}}{p^{2}-m_{4}^{2}+i \Gamma m_{4}}
\end{array}
$$


In the same manner, all the other associated two-point form factors can be identified with the single pole exchange forms of the self-energies.

$$
\begin{aligned}
& \Pi_{a}(p)=\frac{F_{\rho}^{2}}{p^{2}-m_{\rho}^{2}+i \Gamma_{\rho} m_{\rho}} \\
& \Pi_{\hat{a}}(p)=\frac{f_{\pi}^{2}}{p^{2}}+\frac{F_{a}^{2}}{p^{2}-m_{a}^{2}+i \Gamma_{a} m_{a}} \\
& \Pi_{\mathbf{4}}^{L}(p)=\left|y_{L}\right|^{2} \frac{f_{4}^{2}}{p^{2}-m_{4}^{2}+i \Gamma_{4} m_{4}}, \quad L \leftrightarrow R \\
& \Pi_{\mathbf{1}}^{L}(p)=\left|y_{L}\right|^{2} \frac{f_{1}^{2}}{p^{2}-m_{1}^{2}+i \Gamma_{1} m_{1}}, \quad L \leftrightarrow R \\
& M_{\mathbf{4}}(p)=y_{L} y_{R}^{*} m_{4} \frac{f_{4}^{2}}{p^{2}-m_{4}^{2}+i \Gamma_{4} m_{4}} \\
& M_{\mathbf{1}}(p)=y_{L} y_{R}^{*} m_{1} \frac{f_{1}^{2}}{p^{2}-m_{1}^{2}+i \Gamma_{1} m_{1}}
\end{aligned}
$$

The goal when studying the MCHM5 will be to match the effective lagrangian (3.44) to the expressions (3.47)-(3.52). In Chapter 4, we will address this issue accordingly by explicitly computing the form factors.

\subsection{Effective Field Theory Matching}

An effective field theory is defined as a local lagrangian that respects the symmetries of a system. The operators can be of any dimension in this lagrangian, but we assume the EFT is well defined and the irrelevant operators suppressed by the cutoff of the theory.

The EFT can be useful in translating the form factor effects into a known framework. Since form factors are a non-local description (i.e. the operators are not linear in the lagrangian), to match with the EFT, we have to expand them out and find linear structures in the momentum. 
As an example, consider the following lagrangian ${ }^{3}$.

$$
\mathcal{L}=-\frac{1}{2} \phi\left(p^{2}-M^{2}\right) \phi-\frac{1}{2} \pi\left(p^{2}-m^{2}\right) \pi+\frac{\lambda}{4} \phi \pi^{2}
$$

where $M>>m$.

Now, we desire to integrate out the heavy field $\phi$ using its equation of motion

$$
-\left(p^{2}-M^{2}\right) \phi+\frac{\lambda}{2} \pi^{2}=0
$$

Then the remaining theory is non-local and bears a strong resemblance to our form factors.

$$
\mathcal{L}_{\text {form }}=-\frac{1}{2} \pi\left(p^{2}-M^{2}\right) \pi+\frac{\lambda^{2}}{8} \pi^{2} \underbrace{\frac{1}{p^{2}-M^{2}}}_{=\Pi\left(p^{2}\right)} \pi^{2}
$$

Notice that if $p^{2}<<M^{2}$, we are allowed to recover a local description

$$
\mathcal{L}_{E F T}=-\frac{1}{2} \pi\left(p^{2}-M^{2}\right) \pi+\frac{\lambda^{2}}{8}\left(\frac{\pi^{4}}{M^{2}}-\pi^{2} \frac{p^{2}}{M^{4}} \pi^{2}+\ldots\right)
$$

In general, we can write for $p^{2}<<\Lambda^{2}$ :

$$
\Pi\left(p^{2}\right)=\Pi(0)+\frac{p^{2}}{\Lambda} \Pi^{\prime}(0)+\frac{p^{4}}{\Lambda^{4}} \Pi^{\prime \prime}(0)+\ldots
$$

The expansion (3.57) allow one to pass from the non-local formulation of the spectral decomposition to the EFT local description. Historically, this type of expansion was crucial to understanding the electroweak precision measurements [44]. Here, we will use such a tool to review the obtention of oblique parameters in CHMs.

\footnotetext{
${ }^{3}$ Example based on [74]
} 


\subsection{Higher Point Functions}

Throughout this chapter, all of the constructions we have worked with are for two-point functions. The spectral decomposition, narrow resonances and sum rules are applied to functions of only one momentum $p^{\mu}$. Additionally, because of the Lorentz properties of field theories, these functions must be functions exclusively of the Lorentz invariant quantity $p^{2}$.

$$
\Pi\left(p^{\mu}\right)=\Pi\left(p^{2}\right)
$$

However, most of the phenomenological opportunities we will encounter will come from higher point functions. The enriched phenomenology occurs because there are more kinematical possibilities when studying off-shell processes, as we will carefully attain.

The excuse for not wholly discussing the general properties of 3 and 4 point functions is simply because not much is known about them. The complications are several, kinematic dependence on external momenta is much more intricated, and the spectral correspondence is not clearly defined anymore.

In the case of the form factor appearing in the Yukawa interaction, we have a 3-point correlator. Here, the momentum dependence will be on both momenta $p^{\mu}, q^{\nu}$.

$$
M(p, q)=i \int d^{4} x d^{4} y e^{i p . x} e^{i q . y}\left\langle\mathcal{T} \Sigma(0) \overline{\psi_{a}(x)} \psi_{b}(y)\right\rangle
$$

Building the function from only Lorentz invariant quantities lead to the dependence on only three combinations of the four-vectors.

$$
M\left(p^{\mu}, q^{\nu}\right)=M\left(p^{2}, q^{2}, p \cdot q\right)
$$

The strategy to contour this difficulty is to rely only on explicit realizations of three-point functions. These will be constructed solely from the interactions hypothesis between the composite and elementary sectors, and are specific implementations 
of individual UV pictures.

Luckily, the nature of the Higgs will grant us a clue. Because of the Higgs mechanism, the three-point functions will be directly related to the two-point ones since the Higgs vev generate all mass terms. This consequence of the Higgs mechanism will impose boundary conditions on the form factors and constrain the fully dynamical Higgs behaviour in the three-point interactions.

In summary, this chapter was devoted to the analytical behaviour of interactions. The two-point functions analytic properties were directly related to the spectrum of the theory through the spectral decomposition. Such representation has provided a powerful tool for discovering the general expression a form factor can assume.

Because of this construction, the momentum dependence of the interactions will be reflected in the structure of the form factors. An evident influence of the dynamical contributions of the strong sector will appear when we use this approach, and through the next chapters, we will carry the study of the Higgs compositeness fully. 



\section{Chapter 4}

\section{Composite Higgs Form Factors}

We have gathered enough information to explore the composite Higgs boson through form factors. Now, the issue is to transform the Higgs propagation and interaction issues into the analytical properties study of two- and three-point functions.

As we have seen, the two-point function is described through the spectral representation. It provides a correspondence of physical spectral properties with the analyticity of external momenta. This relation fixes a generic picture of the composite Higgs propagation modifications. However, dealing with Higgs interactions, in general, is not so straightforward since the general structure of three-point functions is unknown.

Initially, this characterization will be done by providing simple ansatz expressions for the form factors. They will help to illustrate some particular features on how to handle form factors. Additionally, this discussion will help the build-up to the restoration of the full momentum dependence in the pNGB Composite Higgs Models.

MCHM5's form factors prescription becomes a clear matter after figuring out the partial compositeness and vector partners recipe for the generation of dynamical couplings. The hypothesis on $\mathcal{L}_{\text {int }}$ is essential guidelines to build the complete threepoint form factors.

Then, this formalism can be matched with the correspondent EFT with $d \geq 4$ operators. We shall see that form factors are capable of capturing effects beyond 


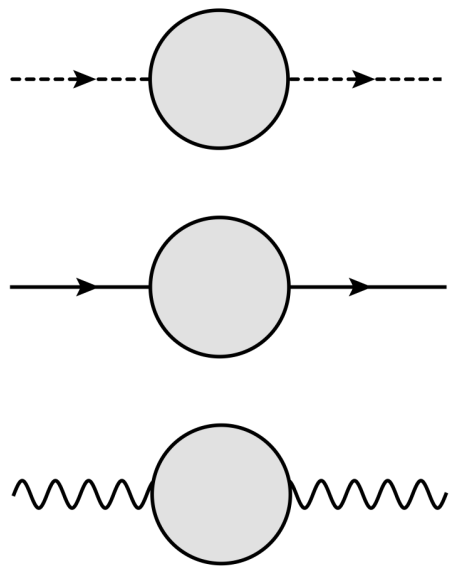

Figure 4.1: Form factor contributions to two-point functions.

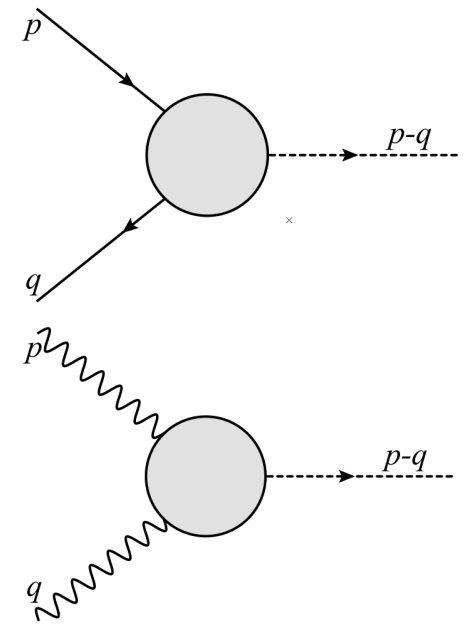

FiguRE 4.2: Form Factor contributions to three-point functions.

the local EFT. Importantly, this discussion reveals that the momentum dependent effects can be are competitive with the modifications to the zero momentum theory ${ }^{1}$.

The discussion of the form factors become most relevant when taking into account the Higgs three-point interactions. Ultimately, the vertices of Higgs interactions are produced by the exchange of composite states, which are integrated out but maintained as non-local form factors expressions. The point functions we are interested in are depicted in figure 4.1-4.2.

\subsection{Simple Form Factor Ansatzes}

As an instructive exercise, let us imagine some simple UV pictures that would result in concrete expressions for the form factors. The goal is to find practical functions for the dynamical Higgs interactions. By beginning with simple form factors, we can illustrate some of the correspondent physical processes underlying the more extensive MCHM5 construction.

\footnotetext{
${ }^{1}$ The misalignment suppressions.
} 
Thematically, this discussion illustrates an essential point of our work. With all its assumptions, the so-called CHMs are just realizations of a composite Higgs. We could imagine that the Higgs compositeness could manifest in a landscape of UV scenarios, a theme for discussion in chapter 6 . For now, we shall focus on discussing some examples of two- and three-point functions form factor structure.

\subsubsection{Two-Point Form Factors}

Two-point form factors capture corrections to the propagation of particles in interacting theories. The two external legs share the same momentum $p^{\mu}$. Because of such dependence, two-point form factors can only be functions of $p^{2}$, the only Lorentz invariant combination.

Guessing some heavy resonance content, as long as its interactions with elementary sector particles, will lead to explicit form factor expressions.

\section{Heavy Scalar Mode Mixing with the Higgs}

A massive scalar mode is a particle with the same quantum numbers of the Higgs boson, but much more massive. We also assume that the Higgs and the heavy scalar interact due to a non-zero mixing between the two.

The general approach to such mixing would be the mass matrix diagonalization. However, one must be careful here.

To define the theory in terms of the mass eigenstates, it is necessary to resolve the mixing at some fixed momentum point. Usually, we define this point to be at the on-shell limit. So, when processes have on-shell external momentum, the interactions are diagonal. However, allowing off-shell form factor legs will generally introduce again mixed interactions. This has far-reaching consequences for the form factor formulation.

The consequence is that the Higgs is still subjected to interaction effects when it is off-shell. 
More concretely, imagine that we integrated out this heavy scalar but kept their momentum dependence through the form factor $\Gamma\left(p^{2}\right)$. Choosing the linear realization for simplicity and passing to momentum space, the induced form for the two-point lagrangian of the light scalar is:

$$
\mathcal{L}=\frac{1}{2}\left(p^{2}-m^{2}\right) \phi^{2}+\Gamma\left(p^{2}\right) \phi^{2}+\mathcal{O}\left(\phi^{4}\right)
$$

Accordingly, the propagator assumes the form:

$$
G\left(p^{2}\right)=\frac{i}{p^{2}-m^{2}+\Gamma\left(p^{2}\right)}
$$

which is similar to the exact propagator (3.9) if we identify the self-interactions contributions with the form factor of a heavy scalar exchange.

There are boundary conditions to the physical mass and the wavefunction renormalization constant. The pole of the propagator defines the physical mass of the lighter states.

$$
m_{P}^{2}=\Gamma\left(m_{P}^{2}\right)-m^{2}
$$

By expanding $\Gamma\left(p^{2}\right)$ around $m_{P}^{2}$, we get

$$
\begin{aligned}
G\left(p^{2}\right) & =\left(p^{2}-m^{2}-\Gamma\left(m_{P}^{2}\right)+\frac{\partial \Gamma\left(m_{P}^{2}\right)}{\partial p^{2}} p^{2}+\mathcal{O}\left(p^{4}\right)\right)^{-1} \\
& \simeq \frac{i}{Z p^{2}-m_{P}^{2}}=\frac{i Z}{p^{2}-{\overline{m_{P}}}^{2}}
\end{aligned}
$$

where ${\overline{m_{P}}}^{2}$ is the redefined physical mass. The value of the wavefunction renormalization constant $Z$ is fixed by the renormalization condition:

$$
\left.\frac{\partial \Gamma\left(p^{2}\right)}{\partial p^{2}}\right|_{p^{2}=m_{P}^{2}}=Z-1
$$

Relations (4.3)-(4.6) define the normalization of the form factor. When $p^{2}=$ $m_{P}^{2}$, the light scalar boson is on-shell, and all the momentum dependent effects due 
to compositeness are absent. This defines the zero momentum limit ${ }^{2}$.

Because of the normalization, we will obtain information from the composite sector only through off-shell effects. Thus, off-shellness of the Higgs is a critical probe to compositeness effects since one is allowed to access the high momentum structure of the theory.

The form factor corresponds to a heavy scalar $(\sigma)$ exchange, that appears as a monopole term. In the large-N limit the narrow width approximation is expected to be valid and we can write:

$$
\Gamma\left(p^{2}\right)=\frac{i F_{\sigma}^{2}}{p^{2}-m_{\sigma}^{2}+i \Gamma_{\sigma} m_{\sigma}}
$$

The off-shell effects of form factor are captured through the mass running of the Higgs. However, this is not the most sensitive physical observable in phenomenological terms. We shall leave the phenomenological possibilities for chapter 5 , focusing now on building up the form factor formalism.

\section{Fermionic and Bosonic Mixings with Heavy Partners}

Similarly to the heavy scalar mode influence in the Higgs propagation, the Fermion and Gauge Boson lines can also gain form factors due to the presence of heavy partners. However, since there the kinetic and mass terms in the lagrangian shared the same symmetries, we were limited to use one form factor. The situation is a bit different for fermions and gauge bosons now.

In this case, chiral symmetry and gauge invariance forbid the explicit appearance of explicit mass terms in the lagrangian. The point is, we have a different symmetry content between kinetic and mass terms since the Higgs mechanism generates the mass ones. So, in general, expect the assignment different form factor expressions for each operator.

\footnotetext{
${ }^{2}$ Since we are referring to the two-point function, the concept of momentum exchange is illdefined. Here zero momentum exchange shall be referred to the on/off-shell properties of the process. This nomenclature is used for unifying the description of compositeness effects for the 3 -point interaction later on.
} 
Beggining with the fermions

$$
\mathcal{L}_{F}=\Pi_{L}\left(p^{2}\right) \bar{q}_{L} \not p q_{l}+\Pi_{R}\left(p^{2}\right) \bar{t}_{R} \not p t_{R}+\Gamma_{F}\left(p^{2}\right) \bar{q}_{L} t_{R}+\text { h.c. }
$$

where $\Pi_{L}\left(p^{2}\right), \Pi_{R}\left(p^{2}\right)$ and $\Gamma_{F}\left(p^{2}\right)$ are the form factors generated through integrating out the left and right-handed heavy fermion partners.

We can redefine the fermion fields in order to canonically normalize the kinetic terms.

$$
q_{L} \rightarrow \frac{e^{i \phi_{L}}}{\sqrt{\left|\Pi_{L}(p)\right|}} q_{L} \quad q_{R} \rightarrow \frac{e^{i \phi_{R}}}{\sqrt{\left|\Pi_{R}(p)\right|}} t_{R}
$$

here, the phase factors are included to make the argument of the square roots real.

Now the lagrangian can be redefined in terms of only one form factor $M(p)$.

$$
\begin{gathered}
\mathcal{L}_{F}=\bar{q}_{L} \not p q_{l}+\bar{t}_{R} \not p t_{R}+\underbrace{\left[\frac{e^{i\left(\phi_{L}+\phi_{R}\right)} \Gamma_{F}(p)}{\sqrt{\left|\Pi_{L}(p)\right|} \sqrt{\left|\Pi_{R}(p)\right|}}\right]}_{\equiv M(p)} \bar{q}_{L} t_{R}+\text { h.c. } \\
\mathcal{L}_{F}=\bar{q}_{L} \not p q_{l}+\bar{t}_{R} \not p t_{R}+M(p) \bar{q}_{L} t_{R}+\text { h.c. }
\end{gathered}
$$

From now, the structure is similar to the heavy scalar exchange. The propagator is

$$
G_{F}(p)=\frac{i}{\not p-M(p)}
$$

We can define the normalization of the form factor from the pole mass relation, and the residue defines the wavefunction normalization.

$$
M\left(m_{P}\right)=m_{P}
$$

Accordingly, the same procedure applies to the Gauge Bosons.

$$
\mathcal{L}_{V}=\left(\eta_{\mu \nu}-\frac{p^{\mu} p^{\nu}}{p^{2}}\right)\left[\Pi_{a}\left(p^{2}\right) A_{\mu}^{a} A_{\nu}^{a}+\Pi_{\hat{a}}\left(p^{2}\right) A_{\mu}^{\hat{a}} A_{\nu}^{\hat{a}}\right]+\Gamma_{V}^{I}\left(p^{2}\right) A_{\mu}^{I} A^{I, \mu}
$$


where $a, \hat{a}$ and $I$ are the indices of the broken, unbroken and all $\mathcal{G}$ generators respectively. $\Pi_{a}, \Pi_{\hat{a}}$ and $\Gamma_{V}^{I}$ are the form factors.

Redefining the fields as

$$
A_{\mu}^{a} \rightarrow \frac{1}{\Pi_{a}(p)} A_{\mu}^{a} \quad A_{\mu}^{\hat{a}} \rightarrow \frac{1}{\Pi_{\hat{a}}(p)} A_{\mu}^{\hat{a}}
$$

will induce the lagrangian:

$$
\begin{gathered}
\mathcal{L}_{V}=\left(\eta_{\mu \nu}-\frac{p^{\mu} p^{\nu}}{p^{2}}\right)\left[A_{\mu}^{a} A_{\nu}^{a}+A_{\mu}^{\hat{a}} A_{\nu}^{\hat{a}}\right]+\underbrace{\frac{\Gamma_{V}^{a}\left(p^{2}\right)}{\prod_{a}\left(p^{2}\right)}}_{\equiv M_{V}^{a}\left(p^{2}\right) / 2} A_{\mu}^{a} A^{a, \mu}+\underbrace{\frac{\Gamma_{V}^{\hat{a}}\left(p^{2}\right)}{\prod_{\hat{a}}\left(p^{2}\right)}}_{\equiv M_{V}^{\hat{a}}\left(p^{2}\right) / 2} A_{\mu}^{\hat{a}} A^{\hat{a}, \mu} \\
\mathcal{L}_{V}=-\frac{1}{4} F_{\mu \nu}^{a} F^{a, \mu \nu}+\frac{M_{V}^{I}\left(p^{2}\right)}{2} A_{\mu}^{I} A^{I, \mu}
\end{gathered}
$$

Again, the pole condition for gauge bosons masses determine the normalization of the form factor $M_{V}^{I}\left(p^{2}\right)$.

$$
M_{V}^{I^{2}}\left(m_{V, I}^{2}\right)=m_{V, I}^{2}
$$

To sum up, the form factors after normalizing the kinetic terms are

$$
\begin{aligned}
& M_{F}(p)=\frac{e^{i\left(\phi_{L}+\phi_{R}\right)} \Gamma_{F}(p)}{\sqrt{\left|\Pi_{L}(p)\right|} \sqrt{\left|\Pi_{R}(p)\right|}} \\
& M_{V}^{A}\left(p^{2}\right)=2 \frac{\Gamma_{V}^{A}\left(p^{2}\right)}{\Pi_{A}\left(p^{2}\right)}
\end{aligned}
$$

Importantly, the two-point form factors are the main indications of propagation modifications due to compositeness. Their influence appears when there are far offshell intermediate contributions in physical processes. Because of this feature, the main observable for these effects is the direct running of the mass parameters, which are difficult experimental signatures [11]. 


\subsubsection{Three-Point Form Factors}

Because of the simple external dependence of two-point form factors on only $p^{2}$, its applications are limited in phenomenological terms. Three-point interactions offer a much more distinctive modification, generating non-local dynamical interactions of the Higgs. The extra momentum dependence due to the presence of another external leg will be important to extend the form factor phenomenological possibilities later on.

\section{Heavy Scalar Monopole}

When introducing interactions between the composite and elementary fermions in Chapter 2, we discussed two types of operators, $\mathcal{O}_{S}$ and $\mathcal{O}_{F}$. The first corresponds to a scalar operator that couples bilinearly with two chiral fermions and the second the PC prescription used in CHMs to generate Higgs-fermion couplings.

Back then, the lagrangian was

$$
\mathcal{L}_{\text {int }}^{\text {bilinear }}=\lambda_{s} \bar{q}_{L} \mathcal{O}_{S}^{c} t_{R}+\text { h.c. }
$$

in which the scalar $\mathcal{O}_{S}$ operator had the same quantum numbers of the Higgs.

$$
\left\langle 0\left|\mathcal{O}_{S}(x)\right| h\right\rangle \neq 0
$$

The explicit realization of this operator is the inclusion of a field that acts as a Heavy scalar mode. This scalar mixes with the Higgs intermediating the bilinear coupling to elementary fermions. Now, we can generate the Higgs Yukawa interaction with a form factor that has the monopole heavy scalar structure. 


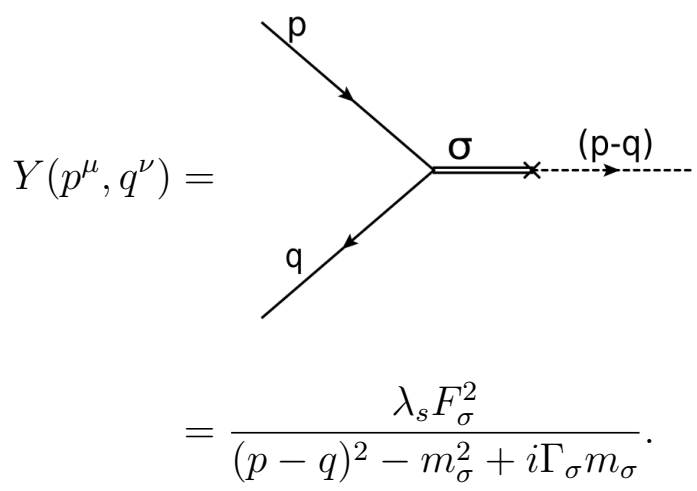

where we assumed the constant $\lambda_{s}$ to control the Heavy scalar/Higgs mixture

Such form factor bears a considerable resemblance with the pion form factor, where the Heavy scalar plays the role of the $\rho$ resonance. Another source of the same structure is technicolor, in which the heavy state arrases as a bound-state of techniquarks $\langle\bar{\psi} \psi\rangle$.

In general, Lorentz invariance imposes the three point vertex to be a function of the variables $p^{2}, q^{2}$ and $p \cdot q$.

$$
Y\left(p^{\mu}, q^{\nu}\right)=Y\left(p^{2}, q^{2}, p \cdot q\right)
$$

In the non-local effective lagrangian, such form factor appears as the momentum dependent coefficient of the Higgs Yukawa terms.

$$
\mathcal{L}=Y\left(p^{2}, q^{2}, p \cdot q\right) \bar{q}_{L} H t_{r}+\text { h.c. }
$$

The role of the Yukawa vertices in the SM is to generate the masses of all charged particles. This situation occurs when the Higgs assumes its vev and becomes non-dynamical, demanding $(p-q)^{\mu}=0$. Therefore, the three-point function is effectively reduced to a two-point one, with only one momentum dependence $p^{2}$.

$$
\left.Y\left(p^{2}, q^{2}, p \cdot q\right)\right|_{p=q}=Y\left(p^{2}\right)=\frac{\sqrt{2} M\left(p^{2}\right)}{v}
$$


This is the Mass-Yukawa relation that is obtained as a consequence of EWSB. At zero trensfered momentum, the form factor (4.27) is reduced to a constant factor that defines the Yukawa constant.

$$
\left.Y\left(p^{2}, q^{2}, p \cdot q\right)\right|_{p-q=0}=\frac{\lambda_{s} F_{\sigma}^{2}}{-m_{\sigma}^{2}+i \Gamma_{\sigma} m_{\sigma}} \equiv y_{f}=\frac{\sqrt{2} m_{t}}{v}
$$

Relation (4.27) imposes the physical constraint that allows us to define the top mass.

In principle, when the Higgs is fully dynamical, one could study this interaction for the on-shell and off-shell cases. However, when the on-shell Higgs condition is satisfied, the momentum is restricted to $(p-q)^{2}=m_{h}^{2}$. As a consequence, the form factor is again reduced to a constant contribution. On-shell Higgs processes only contribute up to a running shift of the top-quark mass. We conclude that all dynamical effects of the heavy scalar are restricted to the off-shell case.

Off-shellness is the ultimate probe to form factor phenomenology. It is capable of producing effects beyond the running of parameters. The central point of the formulation will be an actual modification in the kinematic distributions that reflect the momentum dependence of interactions.

The heavy scalar phenomenology is interesting when the Higgs is off-shell, since the form factor couples with Higgs momentum. However, the actual pNGB models use a different structure for introducing these couplings - they assume partially composite interactions. These will explore the off-shellness of the top external lines instead of the Higgs.

\section{Heavy Fermion Monopole}

Instead of mediating the Higgs interactions with the two elementary scalars via a Heavy scalar, we can assume the nondiagonal PC couplings. 


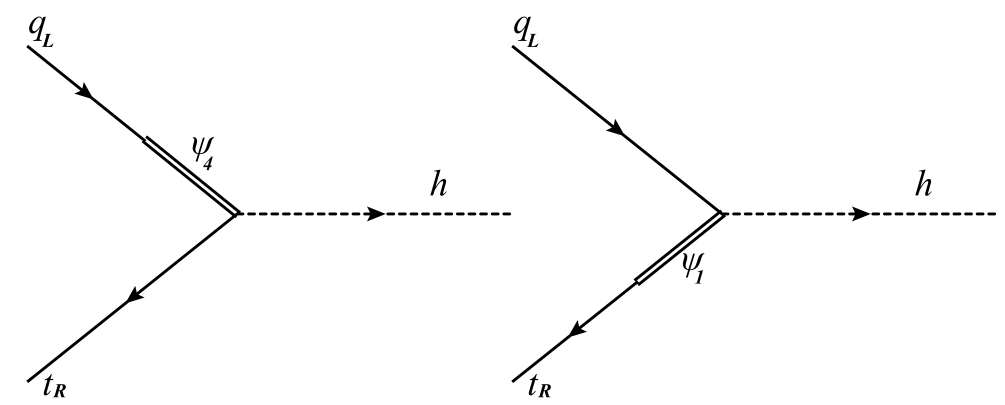

These were generated by the linear $d=5 / 2$ fermionic operator $\mathcal{O}_{F}$ according to the PC prescription of chapter 2 .

$$
\mathcal{L}_{\text {int }}^{\text {linear }}=\lambda_{f}^{L} \bar{q}_{L} \mathcal{O}_{F, L}^{c}+\text { h.c. }
$$

Assuming that the partial composite couplings generate the monopole structure of the form factor, we can include their effects by assuming the two resonances pole structure.

$$
F\left(p^{2}, q^{2}\right) \sim \frac{g M_{1}^{2}}{p^{2}-M_{1}^{2}+i \Gamma_{1} M_{1}}+\frac{g M_{4}^{2}}{q^{2}-M_{4}^{2}+i \Gamma_{4} M_{4}}
$$

The analytic structure of such expression is pictured in figure 4.3. As we shall see, the MHCM5 uses exactly this prescription. In the next section we will proceed treat them properly, deriving the structure from the CCWZ formalism.

\section{Heavy Vectors Dipole}

Similarly to the previous cases, the generation of the Higgs to gauge bosons couplings is done through the mixings with heavy vector states. However, gauge invariance forbids the appearance of nondiagonal terms to generate a monopole.

The gauge construction favours a direct mixing of the elementary vectors with the heavy ones, and the form factor is produced with a dipole term:

$$
F\left(p^{2}, q^{2}\right)=\frac{g M^{2}}{p^{2}-M^{2}+i \Gamma M} \frac{g M^{2}}{q^{2}-M^{2}+i \Gamma M}
$$



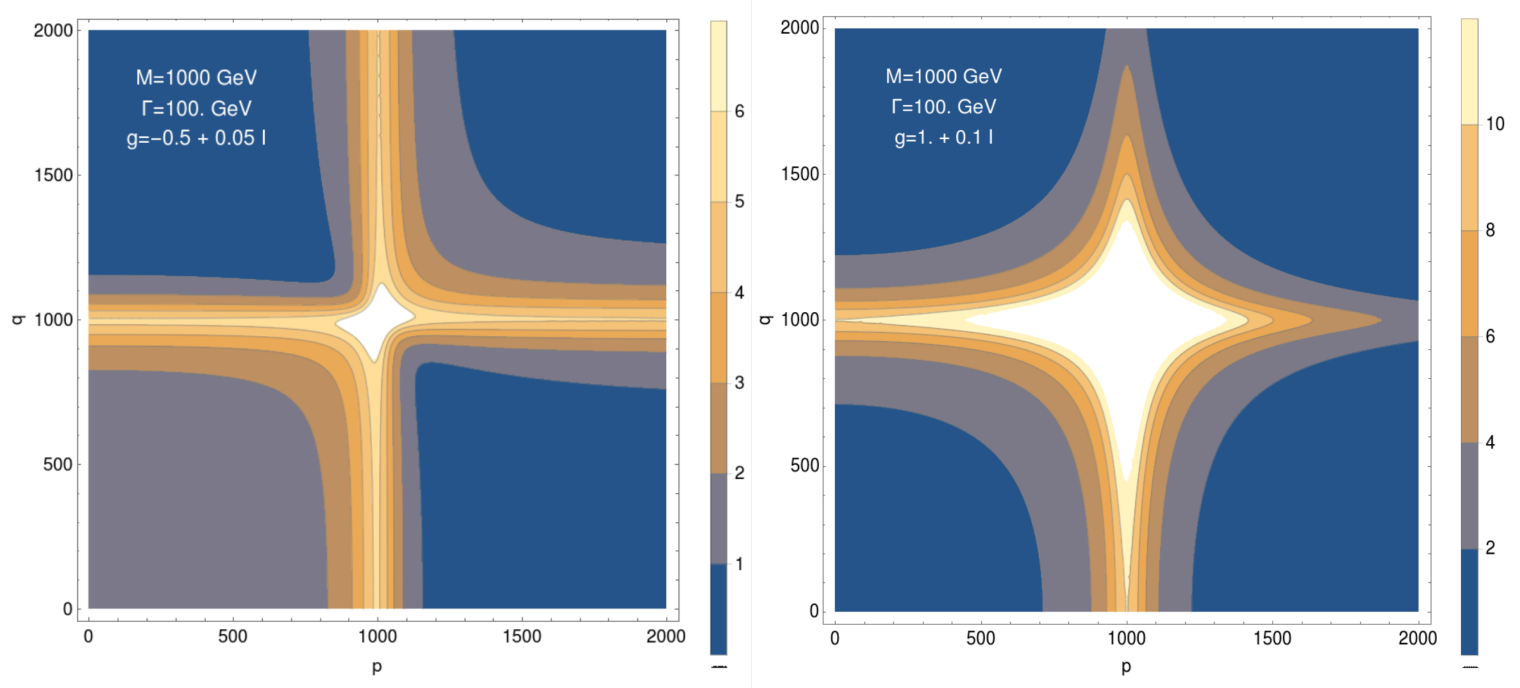

Figure 4.3: Analytic structure of a fermionic monopole (left) or a vector dipole (right). Here we plot the absolute value of the form factors (4.30)-(4.31), by their two momenta $p, q$. Each of these momenta corresponds to a fermion/vector external line.

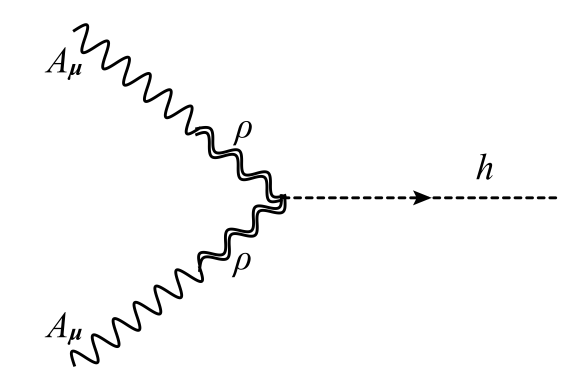

Also, such structure is reproduced in the MCHM5 model. Then, we shall proceed to the characterization of this more robust case.

\subsection{The $\mathrm{MCHM}_{5}$ Form Factors}

In chapter 2, we built the $\mathrm{MCHM}_{\mathbf{5}}$ as an linear effective description. There, the Higgs interactions were modified with respect to the SM by a misalignment suppression. Such shifts are are the first consequence of the presence of a strong 
sector ahead.

$$
\begin{aligned}
\kappa_{V} & =\frac{g_{h V V}^{C H}}{g_{h V V}^{S M}}=\sqrt{1-\xi} \\
k_{t}^{\mathbf{5}} & \equiv \frac{g_{h t t}^{C H}}{g_{h t t}^{S M}}=\frac{1-2 \xi}{\sqrt{1-\xi}} \approx 1-\frac{3}{2} \xi .
\end{aligned}
$$

Most importantly, we argued there is no change to the shape of kinematic distributions, only altering these through an overall rescaling. We argued that this change in shape is a characteristic indicative of internal structure, that is associated with compositeness.

Now, having discussed the dynamical generation of gauge and Yukawa couplings we can assign the effective CHMs as the zero momentum limit of the form factor theory. Then, at zero momentum, we impose boundary conditions on the form factors. Accordingly, through the exploration of off-shellness, we can access the strong sector contributions at the collider level.

When building the couplings of the Higgs, we found that a consistent way is through a monopole of fermionic partners and a dipole of vector resonances.

The form factors for the $\mathrm{MCHM}_{5}$ will be constructed respecting this Higgs interaction generating mechanisms, which ensures the naturalness features of CHMs. With such interactions, we can provide specific forms for the three-point function that relies on the mixing of composite and elementary states. The aim is to restore the form factor formulation the MCHM5's three-point functions for a fully dynamical Higgs. Then we proceed to compute the form factors accordingly.

\subsubsection{Fermion Form Factor}

Linear couplings between the elementary and composite sector are favoured to bilinears if we use Partial Compositeness. Now, the way forward is to insert the resonance fields explicitly, adopting the $\mathrm{PC}$ prescription in it. 
Remember that the elementary third generation of Quarks were embeeded as an incomplete 5-plet of $S O(5)$, with the left-handed fermions as $\mathbf{4}$ and the righthanded as a singlet.

$$
Q_{L}=\left(\begin{array}{c}
b_{l} \\
-i b_{l} \\
t_{l} \\
i t_{l} \\
0
\end{array}\right) \quad T_{R}=\left(\begin{array}{c}
0 \\
0 \\
0 \\
0 \\
t_{r}
\end{array}\right)
$$

The Higgs is realized non-linearly through the Goldstone matrix. Remeber that after exlicit computation it was:

$$
\Sigma(x)=f\left(\begin{array}{c}
\operatorname{Sin}\left(\frac{\Pi}{f}\right) \hat{\Pi} \\
\operatorname{Cos}\left(\frac{\Pi}{f}\right)
\end{array}\right)
$$

and the gauge bosons were identified by their generators that are a linear combination of unbroken $T^{a}{ }^{\prime}$ s.

In table $\mathrm{X}$, we define the notation, mainly to keep track of the multiple fields we are going to use in this parametrization.

\begin{tabular}{|c|c|}
\hline Assigned Field & Particle Classification \\
\hline$\Sigma(x)$ & NGB's/Higgs \\
$Q_{L}(x)$ & 5-plet of elementary left-handed fermions \\
$T_{R}(x)$ & 5-plet of elementary right-handed fermions \\
$\psi_{1}^{i}(x)$ & Singlet of fermionic resonances \\
$\psi_{4}^{i}(x)$ & Fourplet of fermionic resonances \\
\hline
\end{tabular}


With these degrees of freedom, we can write the lagrangian's kinetic term with the explicit resonances fields.

$$
\begin{aligned}
\mathcal{L}_{C S}= & i \overline{Q_{L}} \not D Q_{L}+i \overline{T_{R}} \not D T_{R}+\sum_{i=1}^{N_{s}} \overline{\psi_{1}^{i}}\left(i \not D-m_{1, i}\right) \psi_{1}^{i} \\
& +\sum_{i=1}^{N_{f}} \overline{\psi_{4}^{i}}\left(i \not D-m_{4, i}\right) \psi_{4}^{i}+\left(D_{\mu} \Sigma^{T}\right) D^{\mu} \Sigma+V_{C W}\left(|\Sigma|^{2}\right)
\end{aligned}
$$

We construct the interaction lagrangian by dressing the incoplemte elementary fields with the Goldstone matrix according to CCWZ, thus granting a $S O(5)$ invariant theory. Now, we are able to identify the correct structure for the generation of the Higgs Yukawa couplings.

$$
\begin{aligned}
\mathcal{L}_{i n t}= & f\left[y_{L 1}\left(\overline{Q_{L}} U[\pi]\right)_{1} \psi_{1}^{i}+y_{L 4}\left(\overline{Q_{L}} U[\pi]\right)_{4} \psi_{4}^{i}\right]+\text { h.c. }+ \\
& +f\left[y_{R 1}\left(\overline{T_{R}} U[\pi]\right)_{1} \psi_{1}^{i}+y_{R 4}\left(\overline{T_{R}} U[\pi]\right)_{4} \psi_{4}^{i}\right]+\text { h.c. }
\end{aligned}
$$

The mixing fermion terms will generate the vertices in Fig4.4.

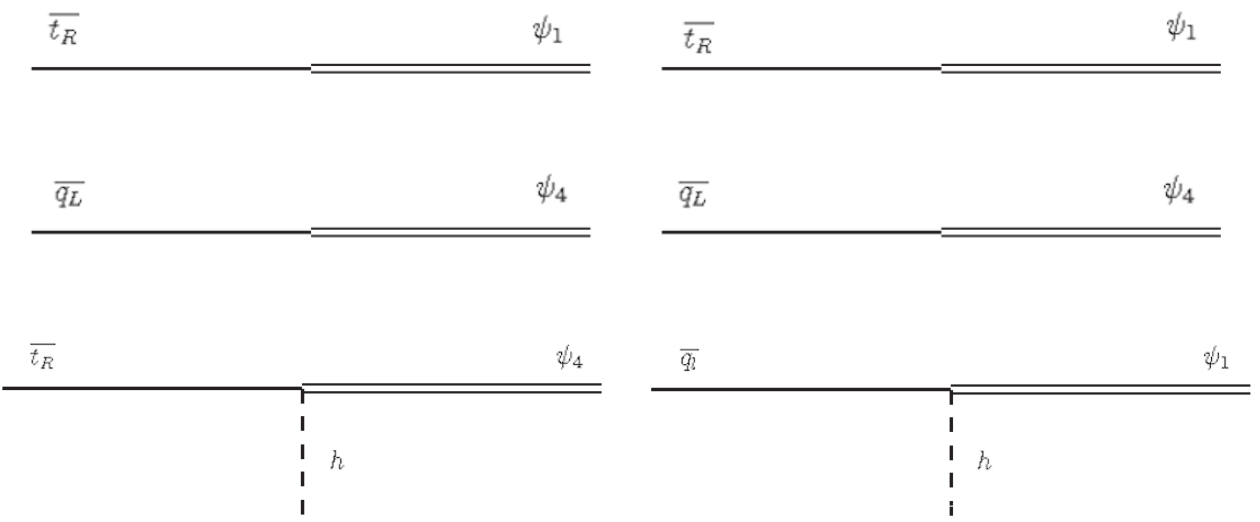

FiguRE 4.4: Fermion vertices deduced from $\mathcal{L}_{\text {Mix }}$. 
And each diagram represent a part of (4.37):

$$
\begin{aligned}
& f y_{R 1}\left(\overline{T_{R}} U[\pi]\right)_{1} \psi_{1}=f y_{R 1} \cos \left(\frac{h}{f}\right) \overline{t_{R}} \psi_{1}=f y_{R 1} \overline{t_{R}} \psi_{1}+\mathcal{O}\left(h^{2}\right) \\
& f y_{L 4}\left(\overline{Q_{L}} U[\pi]\right)_{4} \psi_{4}=f y_{L 4} \cos \left(\frac{h}{f}\right) \overline{q_{L}} \psi_{4}=f y_{L 4} \overline{q_{L}} \psi_{4}+\mathcal{O}\left(h^{2}\right) \\
& f y_{L 1}\left(\overline{Q_{L}} U[\pi]\right)_{1} \psi_{1}=f y_{L 1} \sin \left(\frac{h}{f}\right) \overline{q_{L}} \psi_{1}=y_{L 1} \overline{q_{L}} h \psi_{1}+\mathcal{O}\left(h^{3}\right) \\
& f y_{R 4}\left(\overline{T_{R}} U[\pi]\right)_{4} \psi_{4}=f y_{R 4} \sin \left(\frac{h}{f}\right) \overline{t_{R}} \psi_{4}=y_{R 4} \overline{t_{R}} h \psi_{4}+\mathcal{O}\left(h^{3}\right)
\end{aligned}
$$

Finally, the Yukawa are derived from joining the vertices of (4.38)-(4.41).

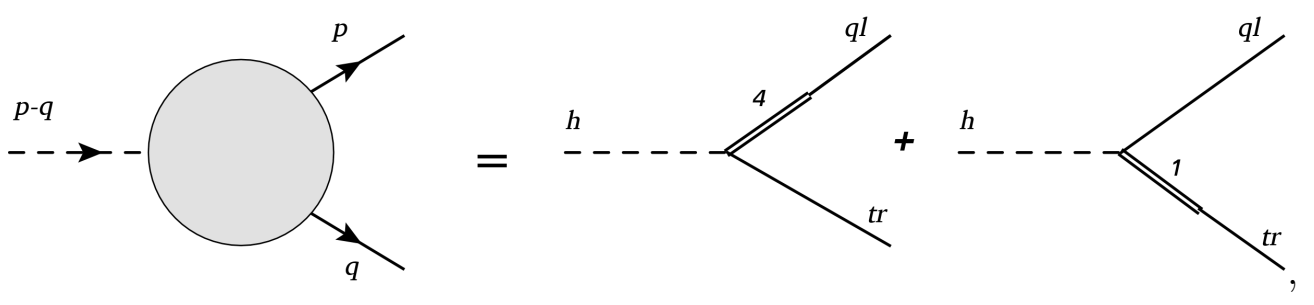

Which assume the form:

$$
M(p, q) \sim M_{4}(p)+M_{1}(q)
$$

Back to the non-linear formalism, the form factors will appear as coefficients of the effective lagrangian. They will introduce a momentum dependence in the interactions of the low energy theory and should be generated according to the couplings prescripted by partial compositeness.

We write once again the the $\mathrm{SO}(5)$ preserving effective lagrangian with the coeffiecients $\Pi_{0}^{L}, \Pi_{1}^{L}, \Pi_{0}^{R}, \Pi_{1}^{R}$ for kinetic terms and $M_{0}, M_{1}$ for Mass/Yukawa terms. Keeping terms up to order $\Sigma^{2}$ we have:

$$
\begin{aligned}
\mathcal{L}_{\text {eff }}^{\mathcal{G}}= & \overline{Q_{L}} \not p\left(\Pi_{0}^{L}(p)+\Pi_{1}^{L}(p, q) \Sigma \Sigma^{T}\right) Q_{L}+ \\
& +\overline{T_{R}} \not p\left(\Pi_{0}^{R}(p)+\Pi_{1}^{R}(p, q) \Sigma \Sigma^{T}\right) T_{R}+ \\
& +\overline{Q_{L}}\left(M_{0}(p)+M_{1}(p, q) \Sigma \Sigma^{T}\right) T_{R}
\end{aligned}
$$


We have to match these coefficients with the form factors defined by the unbroken/broken currents it couples to. This is mainly done by reducing three- to two-point form factors, when the Higgs assume its vev. First, we divide the 5 -plets into 4-plets and the singlet, $Q_{L}=\left(Q_{L 4}, Q_{L 1}\right), T_{R}=\left(T_{R 4}, T_{R 1}\right)$ and $\Sigma_{0}=\left(\Sigma^{4}, \Sigma^{1}\right)$. Opening up (4.43), there will be various combinations of the fields.

$$
\begin{aligned}
\mathcal{L}_{\text {eff }}^{\mathcal{G}}= & \Pi_{0}^{L}(p)\left[\overline{Q_{L 4}} \not p Q_{L 4}+\overline{Q_{L 1}} \not p Q_{L 1}+\overline{Q_{L 4}} \not p Q_{L 1}+\text { h.c. }\right]+Q_{L} \rightarrow T_{R}+ \\
& \Pi_{1}^{L}(p)\left[\overline{Q_{L 4}} \not p \Sigma^{4} \Sigma^{4} Q_{L 4}+\overline{Q_{L 1}} \not p \Sigma^{1} \Sigma^{1} Q_{L 1}+\overline{Q_{L 4}} \not p \Sigma^{4} \Sigma^{1} Q_{L 1}+\text { h.c. }\right]+ \\
& M_{0}\left[\overline{Q_{L 4}} Q_{R 4}+\overline{Q_{L 1}} Q_{R 1}+\overline{Q_{L 4}} Q_{R 1}+\overline{Q_{L 1}} Q_{R 4}\right]+ \\
& M_{1}\left[\overline{Q_{L 4}} \Sigma_{4} \Sigma_{4} Q_{R 4}+\overline{Q_{L 1}} \Sigma_{1} \Sigma_{1} Q_{R 1}+\overline{Q_{L 4}} \Sigma_{4} \Sigma_{1} Q_{R 1}+\overline{Q_{L 1}} \Sigma_{1} \Sigma_{4} Q_{R 4}\right]+\text { h.c. }
\end{aligned}
$$

When the Higgs assumes its vev, we are able to reduce the three point functions contributions in (4.44) to two point ones. By making $\mathcal{G} \rightarrow \mathcal{H}$, we can match the coefficients with the physical currents that define the form factors. Most of the terms in (4.44) are spurious, and in the vacuum $\Sigma \rightarrow \Sigma_{0}=(0,0,0,0,1)$ the effective lagrangian simplified to:

$$
\begin{aligned}
\mathcal{L}_{\text {eff }}^{\mathcal{H}}= & \overline{Q_{L 4}} \not p\left(1+\Pi_{4}^{L}(p)\right) Q_{L 4}+\overline{Q_{L 1}} \not p\left(1+\Pi_{1}^{L}(p)\right) Q_{L 1} \\
& +\overline{T_{R 4}} \not p\left(1+\Pi_{4}^{R}(p)\right) T_{R 4}+\overline{T_{R 1}} \not p\left(1+\Pi_{1}^{R}(p)\right) T_{R 1} \\
& +\overline{Q_{L 4}}\left(M_{4}(p)\right) T_{R 4}+\overline{Q_{L 1}}\left(M_{1}(p)\right) T_{R 1}
\end{aligned}
$$

With the coefficients of (4.45) related to the self-energies $\Pi_{\mathbf{4}}^{L}, \Pi_{\mathbf{4}}^{R}, \Pi_{1}^{L}, \Pi_{1}^{R}$, $M_{4}, M_{1}$ by:

$$
\begin{array}{ll}
\Pi_{0}^{L}(p)=1+\Pi_{\mathbf{4}}^{L}(p), & L \leftrightarrow R \\
\Pi_{1}^{L}(p)=\Pi_{\mathbf{1}}^{L}(p)-\Pi_{\mathbf{4}}^{L}(p), & L \leftrightarrow R \\
M_{0}(p)=M_{4}(p) & \\
M(p, q)=M_{\mathbf{4}}(p)-M_{\mathbf{1}}(q) &
\end{array}
$$


With the usual large-N approximations, we can write the two point funtion contributions as single heavy particle exchange through the form factors $\Pi_{\mathbf{4}}^{L}(p), \Pi_{\mathbf{1}}^{L}(p)$, $M_{4}(p)$ and $M_{\mathbf{1}}(p)$. Each one is associated to a heavy resonance being exchanged in the two-point line.

$$
\begin{aligned}
& \Pi_{\mathbf{4}}^{L}(p)=\left|y_{L 4}\right|^{2} \frac{f^{2}}{p^{2}-m_{4}^{2}+i \Gamma_{4} m_{4}}, \quad L \leftrightarrow R \\
& \Pi_{\mathbf{1}}^{L}(p)=\left|y_{L 1}\right|^{2} \frac{f^{2}}{p^{2}-m_{1}^{2}+i \Gamma_{1} m_{1}}, \quad L \leftrightarrow R \\
& M_{\mathbf{4}}(p)=y_{L 4} y_{R}^{*} m_{4} \frac{f^{2}}{p^{2}-m_{4}^{2}+i \Gamma_{4} m_{4}} \\
& M_{\mathbf{1}}(p)=y_{L 1} y_{R}^{*} m_{1} \frac{f^{2}}{p^{2}-m_{1}^{2}+i \Gamma_{1} m_{1}}
\end{aligned}
$$

Using the explicit expressions (4.50)-(4.50) we have

$$
\begin{aligned}
& \Pi_{0}^{L}(p)=1+\Pi_{4}^{L}(p)=1+\frac{f^{2}\left|y_{L}\right|^{2}}{p^{2}-m_{4}^{2}} \\
& \Pi_{0}^{R}(p)=1+\Pi_{1}^{L}(p)=1+\frac{f^{2}\left|y_{L}\right|^{2}}{p^{2}-m_{1}^{2}} \\
& \Pi_{1}^{L}(p, q)=\Pi_{1}^{L}(p)-\Pi_{4}^{L}(q)=f^{2}\left|y_{L}\right|^{2}\left(\frac{1}{p^{2}-m_{1}^{2}}-\frac{1}{q^{2}-m_{4}^{2}}\right), \quad L \leftrightarrow R \\
& M_{0}(p)=M_{4}(q)=f^{2} y_{L} y_{R} \frac{m_{4}}{p^{2}-m_{4}^{2}} \\
& M(p, q)=M_{\mathbf{4}}(p)-M_{\mathbf{1}}(q)=f^{2} y_{L} y_{R}\left(\frac{m_{4}}{p^{2}-m_{4}^{2}}-\frac{m_{1}}{q^{2}-m_{1}^{2}}\right)
\end{aligned}
$$

where the widths of the resonances are ommited.

In the previous deduction, the objective of to obtain what the form factors (4.54)-(4.58) are explicitly. We have paid extra attention to restoring the dependence on both momenta associated with the external legs. Having matched the correct expressions of the $\mathcal{G}$-invariant lagrangian, we can proceed to calculate the dynamical modifications to the Higgs couplings to fermions. 


\section{Couplings to the Higgs}

Now, consider the fully dynamical Higgs lagrangian $\mathcal{L}_{\text {eff,F }}^{\mathcal{G}}$, and expand the interactions terms up to $d=4$ contributions. Writing (4.43) in the unitary gauge and expanding the general form of $\Sigma(x)$, we have:

$$
\begin{gathered}
\mathcal{L}_{\text {eff,F }}^{\mathcal{G}}=\overline{q_{L}} \not p\left(\Pi_{0}^{L}(p)+\Pi_{1}^{L}(p) \frac{S_{h}^{2}}{2}\right) q_{L}+\overline{t_{R}} \not\left(\Pi_{0}^{R}(q)+\Pi_{1}^{R}(q) C_{h}^{2}\right) t_{R}+ \\
+\overline{t_{R}}\left(M_{1}(p, q) \frac{S_{h} C_{h}}{\sqrt{2}}\right) q_{L}
\end{gathered}
$$

where we have defined $S_{h}=\sin \left(\frac{h+v}{f}\right), C_{h}=\cos \left(\frac{h+v}{f}\right)$.

Now, expanding $S_{h}$ over powers of $h / f$ and normalizing the kinetic terms we get:

$$
\begin{aligned}
& \mathcal{L}_{\text {eff,F }}^{\mathcal{G}}=\overline{q_{L}} \not p q_{l}+\overline{t_{R}} \phi t_{R}+ \\
& +\overline{q_{L}}\left[\frac{1}{\sqrt{2}} \frac{M(p, q) \sqrt{\xi(1-\xi)}}{\sqrt{\Pi_{0}^{L}(p)+\Pi_{1}^{L}(p)\left\langle S_{h}{ }^{2}\right\rangle} \sqrt{\Pi_{0}^{R}(q)+\Pi_{1}^{R}(q)\left\langle C_{h}{ }^{2}\right\rangle}}\right] t_{R} \\
& +\overline{q_{L}}\left[\frac{1}{\sqrt{2}} \frac{M(p, q) \sqrt{\xi(1-\xi)}}{\sqrt{\Pi_{0}^{L}(p)+\Pi_{1}^{L}(p)\left\langle S_{h}{ }^{2}\right\rangle} \sqrt{\Pi_{0}^{R}(q)+\Pi_{1}^{R}(q)\left\langle C_{h}{ }^{2}\right\rangle}}\right] \overbrace{\left(\frac{1-2 \xi}{\sqrt{1-\xi}}\right)}^{=\kappa_{t}^{5}} \frac{1}{\sqrt{\xi}} \frac{h}{f} t_{R} \\
& +\mathcal{O}\left(h^{2} / f^{2}\right) .
\end{aligned}
$$

Defining the form factors as $M_{t}\left(p^{2}\right)$ and $Y_{t}\left(p^{2}, q^{2}\right)$, we have

$$
\mathcal{L}_{\text {eff,F }}^{\mathcal{G}}=\overline{q_{L}} \not p q_{l}+\overline{t_{R}} \phi t_{R}+M_{t}\left(p^{2}\right) \overline{q_{L}} t_{R}+Y_{t}\left(p^{2}, q^{2}\right) \overline{q_{L}} h t_{R}
$$

With the top quark mass is obtained by the pole mass condition $M_{t}\left(m_{t}^{2}\right)=m_{t}$.

$$
m_{t}=\left.\frac{1}{\sqrt{2}} \frac{M \sqrt{\xi(1-\xi)}}{\sqrt{\Pi_{0}^{L}+\Pi_{1}^{L} \frac{1}{2}\left\langle S_{h}{ }^{2}\right\rangle} \sqrt{\Pi_{0}^{R}+\Pi_{1}^{R}\left\langle C_{h}{ }^{2}\right\rangle}}\right|_{p^{2}=m_{t}^{2}}
$$


In the zero transfered momentum limit, the lagrangian of the MCHM5 is recovered.

$$
\mathcal{L}_{\text {eff,F }}^{\mathcal{G}}=\overline{q_{L}} \not p q_{l}+\overline{t_{R}} \phi t_{R}++m_{t} \overline{q_{L}} t_{R}+\left(\frac{1-2 \xi}{\sqrt{1-\xi}}\right) \frac{m_{t}}{v} \overline{q_{L}} h t_{R}
$$

This way we guarantee that the top mass-Yukawa relation is respected with a $\kappa_{t}$ correction due to misaligned EWSB.

$$
y_{t}=\kappa_{t} \frac{m_{t}}{v}=\left(\frac{1-2 \xi}{\sqrt{1-\xi}}\right) \frac{m_{t}}{v}
$$

Satisfying the mass-Yukawa relation is a consequence of the Higgs solution to EWSB. The $\mathrm{CH}$ structure is responsible for a small $\mathcal{O}(\xi)$ deviation from the prediction of the standard model, which was already expected from construction. However, the major modification due to compositeness is reflected in the dependence of the two momenta $p$ and $q$ of the $y_{t}$ form factor.

Recovering the full momentum dependence of the form factor associated with the yukawa interactions, we have the expression:

$$
\begin{aligned}
Y_{t}(p, q) & =\frac{1}{\sqrt{2}} \frac{M(p, q)(1-2 \xi)}{\sqrt{\Pi_{0}^{L}(p)+\Pi_{1}^{L}(p) \frac{1}{2}\left\langle S_{h}^{2}\right\rangle} \sqrt{\Pi_{0}^{R}(q)+\Pi_{1}^{R}(q)\left\langle C_{h}^{2}\right\rangle}} \\
& =\frac{\frac{(1-2 \xi)}{\sqrt{2}} y_{l} y_{r} e^{i\left(\phi_{l}-\phi_{r}\right)}\left[\frac{m_{4}}{p^{2}-m_{4}^{2}}-\frac{m_{1}}{q^{2}-m_{1}^{2}}\right]}{\sqrt{1+\frac{f^{2} y_{l}^{2}}{p^{2}-m_{4}^{2}}+\frac{v^{2} y_{l}^{2}}{2}\left(\frac{1}{p^{2}-m_{1}^{2}}-\frac{1}{p^{2}-m_{4}^{2}}\right)} \sqrt{1+\frac{f^{2} y_{r}^{2}}{q^{2}-m_{1}^{2}}+y_{r}^{2}\left(\frac{f^{2}-v^{2}}{q^{2}-m_{1}^{2}}-\frac{f^{2}-v^{2}}{q^{2}-m_{4}^{2}}\right)}} .
\end{aligned}
$$

We can plot the contours of the absolute value of $Y_{t}(p, q)$ on $p-q$ plane, as is indicated in figure 4.5 .

The complicated analytical expression of the form factor induces a rich structure in the $p-q$ plane. Most importantly, accessing different regions of this plane will grant new possibilities for the phenomenological study of compositeness in CHMs.

Without the constraints, the form factor can have a very diverse momentum dependence. To ground such a study, we will have to take a careful look into the 

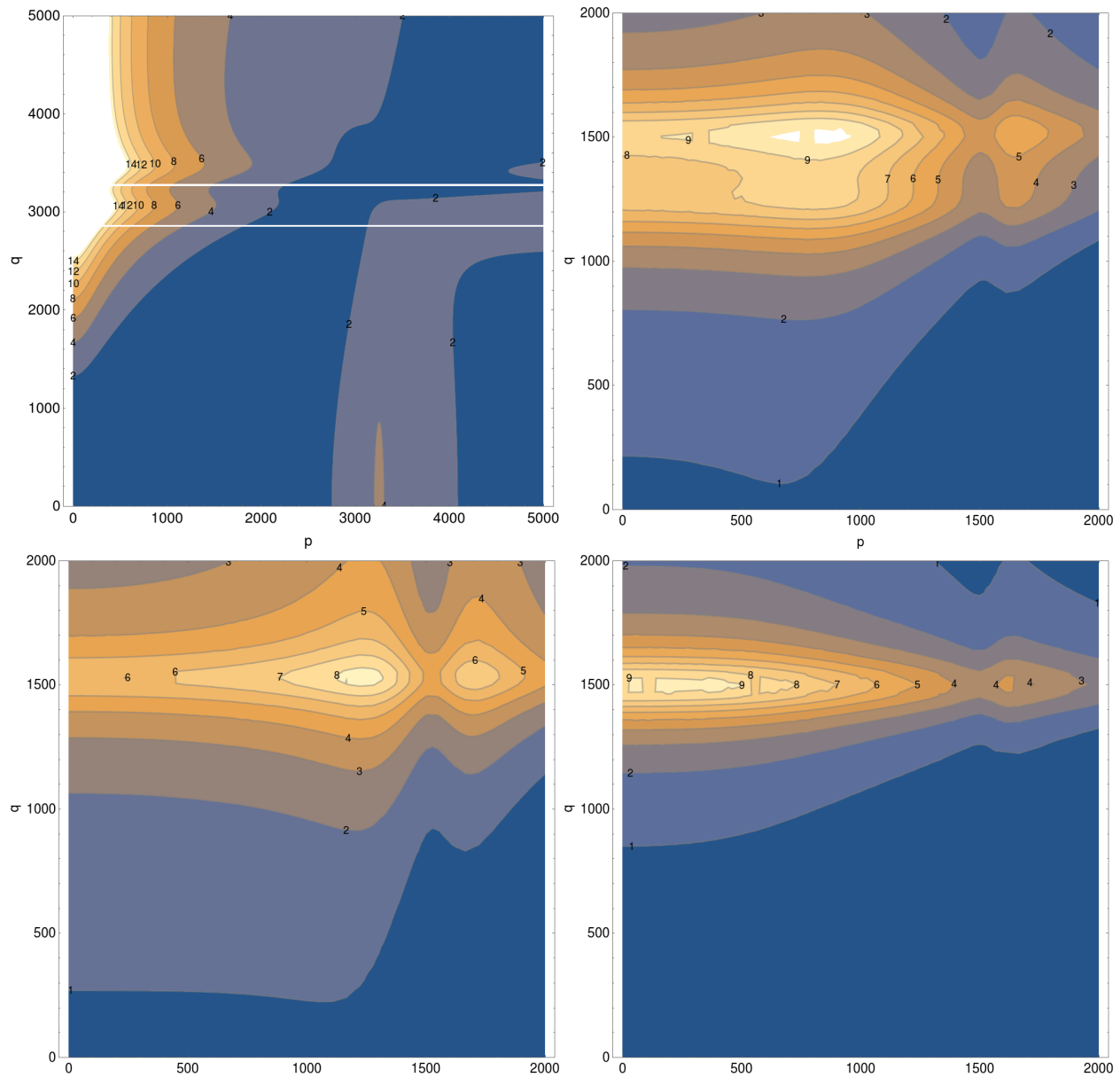

FiguRE 4.5: Absolute value of Yukawa form factor as a function of $\mathrm{p}$ and q. The form factor of the MCHM5 has a complex structure. To study its phenomenology, we shall apply constraints to the expessions derived. 
parameter space of the model. This task will be addressed in chapter 5 , where we shall apply the constraints on the form factor expressions.

\section{Matching with the EFT}

Expanding (4.65) up to first order terms in $\xi$ and keeping only single higgs interactions leads us to

$$
\begin{gathered}
\mathcal{L}_{\text {eff }, F}^{\mathcal{G}}=\overline{q_{L}} \not p\left(\Pi_{0}^{L}(p)+\Pi_{1}^{L}(p) \frac{1}{2}\left[\xi+\frac{2 \xi}{v} h\right]\right) q_{L}+\overline{t_{R}} \phi\left(\Pi_{0}^{R}(q)+\Pi_{1}^{R}(q)\left[(1-\xi)-\frac{2 \xi}{v} h\right]\right) t_{R} \\
+\overline{t_{R}}\left(M_{1}(p, q) \frac{1}{\sqrt{2}}\left[\left(1-\frac{2}{3} \xi\right) v+(1-2 \xi) h\right]\right) q_{L}
\end{gathered}
$$

Now, in order to recover an local EFT we should expand the form factors as functions of the momenta.

$$
\begin{aligned}
\Pi_{0}^{L}(p) & =\Pi_{0}^{L}(0)+\left.\frac{\partial \Pi_{0}^{L}(p)}{\partial\left(p^{2} / f^{2}\right)}\right|_{p=0} \frac{p^{2}}{f^{2}}+\mathcal{O}\left(p^{4} / f^{4}\right) \\
& \equiv \Pi_{0,0}^{L}+\Pi_{0,0}^{L} \frac{p^{2}}{f^{2}} \\
\Pi_{0}^{R}(q) & =\Pi_{0,0}^{R}+\Pi_{0,0}^{R} \frac{q^{2}}{f^{2}} \\
\Pi_{1}^{L}(p) & =\Pi_{1,0}^{L}+\Pi_{1,0}^{L} \frac{p^{2}}{f^{2}} \\
\Pi_{1}^{R}(q) & =\Pi_{1,0}^{R}+\Pi_{1,0}^{R} \frac{q^{2}}{f^{2}} \\
M(p, q) & =f\left(M_{0}+M_{1, p} \frac{p^{2}}{f^{2}}+M_{1, q} \frac{q^{2}}{f^{2}}\right)
\end{aligned}
$$


Rewriting the effective lagrangian

$$
\begin{aligned}
& \left.\mathcal{L}_{E F T, F}=\left[\frac{(1-2 \xi)}{\sqrt{2}} M_{0}\right] v \overline{t_{R}} q_{L}\right\} \rightarrow d=3 \text { operator } \\
& +\underbrace{\left[\Pi_{0,0}^{L}+\left(\frac{\xi}{2} \Pi_{1,0}^{L}\right)\right] \overline{q_{L}} \not p q_{L}+\left[\Pi_{0,0}^{R}+(1-\xi) \Pi_{1,0}^{R}\right] \overline{t_{R}} \phi t_{R}+\left[\frac{\left(1-\frac{2}{3} \xi\right)}{\sqrt{2}} M_{0}\right] \overline{t_{R}} h q_{L}}_{d=4 \text { operators }} \\
& +\underbrace{\left[\xi \Pi_{1,0}^{L}\right] \frac{1}{v} \overline{q_{L}} \not p h q_{L}-\left[2 \xi \Pi_{1,0}^{R}\right] \frac{1}{v} \overline{t_{R}} \phi h t_{R}+\left[\frac{\xi}{\sqrt{2}} M_{1, p}\right] \frac{1}{v} p^{2} \overline{t_{R}} q_{L}+\left[\frac{\xi}{\sqrt{2}} M_{1, q}\right] \frac{1}{v} q^{2} \overline{t_{R}} q_{L}}_{d=5 \text { operators }} \\
& +\underbrace{\left[\xi \Pi_{0,1}^{L}\right] \frac{1}{v^{2}} p^{2} \overline{q_{L}} \not p q_{L}-\left[\xi \Pi_{0,1}^{R}\right] \frac{1}{v^{2}} q^{2} \overline{t_{R}} \phi t_{R}+\left[\frac{\xi}{\sqrt{2}} M_{1, p}\right] \frac{1}{v^{2}} p^{2} \overline{t_{R}} h q_{L}+\left[\frac{\xi}{\sqrt{2}} M_{1, q}\right] \frac{1}{v^{2}} q^{2} \overline{t_{R}} h q_{L}}_{d=6 \text { operators }}
\end{aligned}
$$

Now we redefine the fields to get canonically normalized kinetic terms.

$$
\begin{aligned}
\mathcal{L}_{E F T}^{d \leq 6}= & \overline{q_{L}} \not p q_{L}+\overline{t_{R}} \not p t_{R}+m_{t} \overline{t_{R}} q_{L}+\kappa_{5}^{\xi} y_{t} \overline{t_{R}} h q_{L} \\
& +\frac{c_{k}^{5, L} \xi}{v} \overline{q_{L}} \not p h q_{L} \frac{c_{k}^{5, R} \xi}{v} \overline{t_{R}} \phi h t_{R}+\frac{c_{y}^{5, L} \xi}{v} p^{2} \overline{t_{R}} q_{L}+\frac{c_{y}^{5, R} \xi}{v} q^{2} \overline{t_{R}} q_{L} \\
& +\frac{c_{k}^{6, L} \xi}{v^{2}} p^{2} \overline{q_{L}} \not p q_{L}+\frac{c_{k}^{6, R} \xi}{v^{2}} q^{2} \overline{t_{R}} \phi t_{R}+\frac{c_{y}^{6, L} \xi}{v^{2}} p^{2} \overline{t_{R}} h q_{L}+\frac{c_{y}^{6, R} \xi}{v^{2}} q^{2} \overline{t_{R}} h q_{L}
\end{aligned}
$$

where the coefficients (4.77) are matched to the form factor constants.

$$
\begin{aligned}
& c_{k}^{5, L}=c_{k}^{6, L}=\frac{\Pi_{1,0}^{L}}{\Pi_{0,0}^{L}} \\
& c_{k}^{5, R}=c_{k}^{6, R}=\frac{-2 \Pi_{1,0}^{R}}{\Pi_{0,0}^{R}+\Pi_{1,0}^{R}} \\
& c_{y}^{5, p}=c_{y}^{6, p}=\frac{M_{1, P}}{\sqrt{2} \sqrt{\Pi_{0,0}^{L}} \sqrt{\Pi_{0,0}^{R}+\Pi_{1,0}^{R}}} \\
& c_{y}^{5, q}=c_{y}^{6, q}=\frac{M_{1, q}}{\sqrt{2} \sqrt{\Pi_{0,0}^{L}} \sqrt{\Pi_{0,0}^{R}+\Pi_{1,0}^{R}}}
\end{aligned}
$$


Using the explicit expressions for the form factors, and defining $g_{1}=m_{1} / f$, $g_{4}=m_{4} / f, g_{\Gamma 1}=\Gamma_{1} / f$ and $g_{\Gamma 4}=\Gamma_{4} / f$

$$
\begin{aligned}
c_{k}^{L} & =\frac{y_{l}^{2}\left(g_{1}^{2}-i g_{1} g_{\Gamma 1}-g_{4}\left(g_{4}-i g_{\Gamma 4}\right)\right)}{g_{1}\left(g_{1}-i g_{\Gamma 1}\right)\left(g_{4}^{2}-i g_{4} g_{\Gamma 4}-y_{l}^{2}\right)} \\
c_{k}^{R} & =\frac{2 y_{r}^{2}\left(-g_{1}^{2}+i g_{1} g_{\Gamma 1}+g_{4}\left(g_{4}-i g_{\Gamma 4}\right)\right)}{y_{r}^{2}\left(g_{1}^{2}-i g_{1} g_{\Gamma 1}-2 g_{4}\left(g_{4}-i g_{\Gamma 4}\right)\right)+g_{1} g_{4}\left(g_{1}-i g_{\Gamma 1}\right)\left(g_{4}-i g_{\Gamma 4}\right)} \\
c_{y}^{p} & =-\frac{y_{l} y_{r}}{g_{4}\left(g_{4}-i g_{\Gamma 4}\right)^{2} \sqrt{2-\frac{2 y_{l}^{2}}{g_{4}^{2}-i g_{4} g_{\Gamma 4}}} \sqrt{1+y_{r}^{2}\left(\frac{1}{g_{4}^{2}-i g_{4} g_{\Gamma 4}}-\frac{2}{g_{1}^{2}-i g_{1} g_{\Gamma 1}}\right)}} \\
c_{y}^{q} & =\frac{y_{l} y_{r}}{g_{1}\left(g_{1}-i g_{\Gamma 1}\right)^{2} \sqrt{2-\frac{2 y_{l}^{2}}{g_{4}^{2}-i g_{4} g_{\Gamma 4}}} \sqrt{1+y_{r}^{2}\left(\frac{1}{g_{4}^{2}-i g_{4} g_{\Gamma 4}}-\frac{2}{g_{1}^{2}-i g_{1} g_{\Gamma 1}}\right)}}
\end{aligned}
$$

Expressions (4.82)-(4.85) define the coefficients of the local effective field theory in the limit of $p^{2}<<\Lambda^{2} \sim 4 \pi f$. However, if we wish to match these with the $d=6$ effective operators to a tabled basis sich as the SILH [27, 32] we run into a problem. First of all the $d=3$ and $d=5$ operators we wrote match with the $d=6$ ones if we assume the Higgs can generate a vev in $d=6$ terms. But those remaining are not listed as effective operators and is not clear how do these $p^{2}$ terms behave as a local EFT.

What could be done is to match these operators to a $d=8$ basis. This remark comes with the realization that we can pick a certain $d=8$ operator and generate the terms in (4.77). For example, by performing a partial integration and a going to momentum space, we can

$$
\frac{c_{8}}{\Lambda^{2}}\left|D_{\mu} H\right|^{2} \overline{q_{L}} \not p q_{L} \Rightarrow \frac{c_{k}^{6, L}}{f^{2}} p^{2} \overline{q_{L}} \not p q_{L}
$$

Even though we did not fully explore this approach, this might suggest that the non-linear theory encapsulate non-negligible higher-dimensional operators, that would not be taken into account in order six.

The important point here is that by expanding the form factors we were led to order $p^{2} / f^{2}$ contributions. Thus, at high moments these dynamical effects are 
comparable to the order $\xi$ effects of the zero momentum CHM. We shall see this effect in the phenomenological study of the next chapter.

In summary, the inclusion of momentum dependence in the $M C H M_{5}$ is effectively done using form factors. Such additional dynamical effects are reduced to the usual composite Higgs models suppressions by going into on-shell processes. Importantly, the mass normalization was established at the on-shell limit, and dynamical effects are only expected at the off-shell cases, where the momentum transfer is high enough. 


\subsubsection{Gauge Bosons Form Factor}

Differently from the fermionic case, the inclusion of vector resonances are not done through monopole terms. The picture for gauge boson is that the elementary particles mix directly with their heavy vector partners, which are then connected to the Higgs though bilinear couplings.

A fundamental property required to have a dynamical contribution in gauge boson sector is the presence of two vector multiplets. The first one is associated with the unbroken currents and the other associated with the broken ones.

\begin{tabular}{|c|c|}
\hline Assigned Field & Particle Classification \\
\hline$\Sigma(x)$ & NGB's/Higgs \\
$A_{\mu}^{i}(x)$ & Elementary Gauge Bosons \\
$\rho_{\mu}^{i}(x)$ & Unbroken vector resonances \\
$a_{\mu}^{i}(x)$ & Broken vector resonances \\
\hline
\end{tabular}

The resonances can be introduced explicitly through the CCWZ formalism. As prescripted, these heavy partners interactions with the composite Higgs appears as external gauge sources. To all intents, they appear as extra covariant derivative terms in the explicit sources lagrangian.

$$
\begin{aligned}
\mathcal{L}_{\text {Kin }}= & -\frac{1}{4} A_{\mu \nu} A^{\mu \nu}+\left[-\frac{1}{4} v^{i}{ }_{\mu \nu} v^{i \mu \nu}+\frac{m_{v_{i}}}{2} v_{i}^{\mu} v_{i \mu}\right]+v \leftrightarrow a \\
& +\left(D_{\mu} \Sigma^{T}\right) D^{\mu} \Sigma+V_{C W}\left(|\Sigma|^{2}\right)
\end{aligned}
$$

with $D_{\mu} \Sigma(x)=\left(\partial \mu+i g_{*}^{v} T^{a} v_{\mu}^{a}+i g_{*}^{a} T^{\hat{a}} a_{\mu}^{\hat{a}}\right) \Sigma(x)$. The field $v_{\mu}^{a}$ is assigned to the unbroken resonant currents and $a_{\mu}^{\hat{a}}$ to the broken ones.

The result is that we have a bilinear vertice of two resonances and the Higgs. We will use this to build the Higgs interaction with elementary gauge bosons.

For the purpose of generating the elementary gauge couplings, the introduction of direct mixing of elementary and resonant vectors is crutial. Here, the mixing lagrangian is:

$$
\mathcal{L}_{M i x}=A_{\mu}^{a} v^{a \mu}+A_{\mu}^{\hat{a}} a^{\hat{a} \mu}
$$


With the mixing, the gauge-Higgs interactions are built through a dipole structure. The elementary bosons mix with two vectors that combine to form a Higgs.

Thus, the form factor associated with such interaction should be of the form

$$
\Pi_{1}\left(p^{2}, q^{2}\right)=\frac{f^{2} m_{\rho}^{2} m_{a}^{2}}{\left(p^{2}-m_{\rho}^{2}\right)\left(q^{2}-m_{a}^{2}\right)}
$$

Also, there are contributions to the two-point interactions that come from resonance exchange. These are mainly given according to the spectral distribution. Another contribution comes from a Higgs exchange, which couples to broken current. These will appear as a low momentum pole, signalling Goldstone modes.

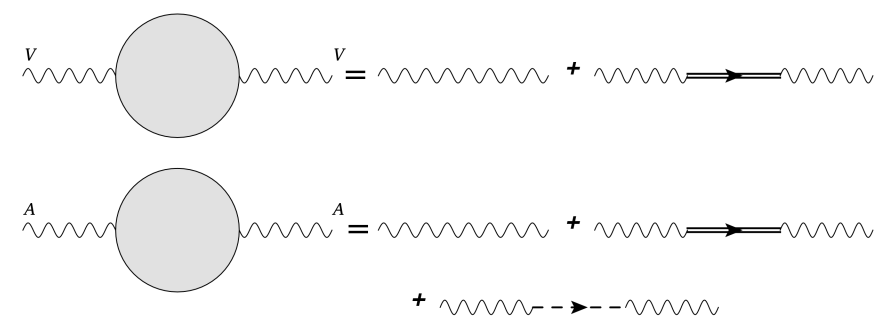

Kepping a dynamical Higgs, the form factor effecive lagrangian up to order $\mathcal{O}\left(\Sigma^{2}\right)$ is

$$
\mathcal{L}_{\text {eff }}^{\mathcal{G}}=\frac{1}{2} \mathcal{P}^{\mu \nu}\left(\Pi_{0}(p) \operatorname{Tr}\left(A_{\mu} A_{\nu}\right)+\Pi_{1}(p, q) \Sigma^{T} A_{\mu} A_{\nu} \Sigma\right)
$$

In the vacuum this becomes

$$
\mathcal{L}_{\text {eff }}^{\mathcal{H}}=\frac{1}{2} \mathcal{P}^{\mu \nu}\left(\Pi_{0}(p) A_{\mu}^{a} A_{\nu}^{a}+\left(\Pi_{0}(p)+\frac{\Pi_{1}(p)}{2}\right) A_{\mu}^{\hat{a}} A_{\nu}^{\hat{a}}\right)
$$

So the $S O(5)$ lagrangian coefficients are related to the form factors $\Pi_{\mathbf{4}}^{L}, \Pi_{\mathbf{4}}^{R}, \Pi_{\mathbf{1}}^{L}, \Pi_{\mathbf{1}}^{R}$, $M_{4}, M_{1}$ by

$$
\begin{aligned}
& \Pi_{0}(p)=\frac{p^{2}}{g^{2}}+\Pi_{a}(p) \\
& \Pi_{1}(p)=2 \Pi_{\hat{a}}(p)-2 \Pi_{a}(p)
\end{aligned}
$$


With the self energies

$$
\begin{aligned}
\Pi_{a}(p) & =\frac{F_{\rho}^{2}}{p^{2}-m_{\rho}^{2}} \\
\Pi_{\hat{a}} & =\frac{F_{a}^{2}}{p^{2}-m_{a}^{2}}
\end{aligned}
$$

The Weinberg sum rules impose a condition to the constants $F_{\rho}$ and $F_{a}$

$$
\left\{\begin{array}{l}
F_{\rho}^{2}-F_{a}^{2}=\frac{f^{2}}{2} \\
F_{\rho}^{2} m_{\rho}^{2}-F_{a}^{2} m_{a}^{2}=0
\end{array}\right.
$$

Resolving the system of equations

$$
\begin{aligned}
& F_{\rho}^{2}=\frac{f^{2}}{2} \frac{m_{a}^{2}}{m_{a}^{2}-m_{\rho}^{2}} \\
& F_{a}^{2}=\frac{f^{2}}{2} \frac{m_{\rho}^{2}}{m_{a}^{2}-m_{\rho}^{2}}
\end{aligned}
$$

Putting everything together, the form factors are:

$$
\begin{aligned}
& \Pi_{0}(p)=p^{2}\left(\frac{1}{g^{2}}+\Pi_{a}(p)\right)=\frac{p^{2}}{g^{2}}+\frac{p^{2}}{2} \frac{f^{2}}{p^{2}-m_{\rho}^{2}} \frac{m_{a}^{2}}{m_{a}^{2}-m_{\rho}^{2}} \\
& \Pi_{1}(p, q)=\frac{f^{2} m_{\rho}^{2} m_{a}^{2}}{\left(p^{2}-m_{\rho}^{2}\right)\left(q^{2}-m_{a}^{2}\right)}
\end{aligned}
$$

where the multiplicative $p^{2}$ factor is due to the kinetic terms definition we adopted.

We can now write the full effective lagrangian with a dynamical Higgs. In the unitary gauge, it is:

$$
\begin{aligned}
\mathcal{L}_{E f f, V}^{\mathcal{G}} & =\frac{1}{2} \mathcal{P}^{\mu \nu}\left[\Pi_{0}(p) \operatorname{Tr}\left(A_{\mu} A_{\nu}\right)+\Pi_{1}(p, q) \Sigma^{T} A_{\mu} A_{\nu} \Sigma\right] \\
& =\frac{1}{2} \mathcal{P}^{\mu \nu}\left[\Pi_{0}(p)\left(A_{\mu}^{a} A_{\nu}^{a}+A_{\mu}^{\hat{a}} A_{\nu}^{\hat{a}}\right)+\Pi_{1}(p, q) A_{\mu}^{A} A_{\nu}^{B} \Sigma^{T} T^{A} T^{B} \Sigma\right]
\end{aligned}
$$


To obtain the gauge boson masses and their couplings to the Higgs, we have to normalize the kinetic terms. First, we switch off the non-SM fields.

$$
\begin{aligned}
\mathcal{L}_{E f f, V}^{\mathcal{G}}= & \frac{1}{2} \mathcal{P}^{\mu \nu}\left[\left(\Pi_{0}(p)+\frac{S_{h}^{2}}{4} \Pi_{1}(p, q)\right) B_{\mu} B_{\nu}\right. \\
& \left.+\left(\Pi_{0}(p)+\frac{S_{h}^{2}}{4} \Pi_{1}(p, q)\right) A_{\mu}^{a} A_{\nu}^{a}-2\left(\frac{S_{h}^{2}}{4} \Pi_{1}(p, q)\right) A_{\mu}^{3} B_{\nu}\right]
\end{aligned}
$$

Now, expanding $\sin ^{2}((h+v) / f) \approx \sin ^{2}(v / f)+2 h / f \sin (v / f) \cos (v / f)$ and assuming that the misalignment angle $\xi$ is small:

$$
\begin{aligned}
\mathcal{L}_{E f f, V}^{\mathcal{H}}= & \frac{1}{2} \mathcal{P}^{\mu \nu}\left[\left(\Pi_{0}+\frac{\left\langle S_{h}^{2}\right\rangle}{4} \Pi_{1}\right)\{\overbrace{A_{\mu}^{1} A_{\nu}^{1}+A_{\mu}^{2} A_{\nu}^{2}}^{2 W_{\mu}^{+} W_{\nu}^{-}}+A_{\mu}^{3} A_{\nu}^{3}+B_{\mu} B_{\nu}\}\right. \\
& \left.-2\left(\frac{\left\langle S_{h}^{2}\right\rangle}{4} \Pi_{1}\right) A_{\mu}^{3} B_{\nu}\right]+2 \kappa_{V} \frac{h}{f} \frac{\Pi_{1}(p, q)}{4}[\overbrace{A_{\mu}^{1} A_{\nu}^{1}+A_{\mu}^{2} A_{\nu}^{2}}^{2 W_{\mu}^{+} W_{\nu}^{-}}+A_{\mu}^{3} A_{\nu}^{3}+B_{\mu} B_{\nu}]
\end{aligned}
$$

Finally, the lagrangian assumes the form:

$$
\begin{aligned}
\mathcal{L}_{V}^{\mathcal{H}}= & \frac{\mathcal{P}^{\mu \nu}}{2}\left[\left(\Pi_{0}+\frac{\left\langle S_{h}^{2}\right\rangle}{4} \Pi_{1}\right) B_{\mu} B_{\nu}+\left(\Pi_{0}+\frac{\left\langle S_{h}^{2}\right\rangle}{4} \Pi_{1}\right) W_{\mu}^{3} W_{\nu}^{3}\right]+ \\
& +\mathcal{P}^{\mu \nu}\left[\left(\Pi_{0}+\frac{\left\langle S_{h}^{2}\right\rangle}{4} \Pi_{1}\right) W_{\mu}^{+} W_{\nu}^{-}-\frac{\Pi_{1}\left\langle S_{h}^{2}\right\rangle}{4} W_{\mu}^{3} B_{\nu}\right] \\
& +\frac{\Pi_{1}(p, q)}{4}\left[2 h W_{\mu}^{+} W_{\nu}^{-}+h A_{\mu}^{3} A_{\nu}^{3}+h B_{\mu} B_{\nu}\right]
\end{aligned}
$$

\section{Electroweak Precision Tests}

Form factors are analytic functions of the external momentum, thus admitting a Taylor expansion. 


$$
\begin{aligned}
\Pi_{0}\left(p^{2}\right) & =\overbrace{\Pi_{0}(0)}^{=0}+\Pi_{0}^{\prime}(0) p^{2}+\mathcal{O}\left(p^{4}\right) \\
& =p^{2}\left\{\frac{1}{g^{2}}+\frac{f^{2} m_{a}^{2}}{2 m_{\rho}^{2}\left(m_{a}^{2}-m_{\rho}^{2}\right)}\right\}+\ldots \\
\Pi_{1}\left(p^{2}\right) & =\overbrace{\Pi_{0}(0)}^{=f^{2}}+\Pi_{0}^{\prime}(0) p^{2}+\mathcal{O}\left(p^{4}\right) \\
& =f^{2}-p^{2}\left\{\frac{f^{2}}{m_{a}^{2}}+\frac{f^{2}}{m_{\rho}^{2}}\right\}+\ldots
\end{aligned}
$$

Now, normalizing the mass terms, the effective lagrangian becomes:

$$
\begin{aligned}
\mathcal{L}_{E f f, V}^{\mathcal{G}}= & \frac{1}{4} A_{\mu \nu}^{i} A^{i \mu \nu}+\frac{1}{4} B_{\mu \nu} B^{\mu \nu}+\overbrace{{\widetilde{\Pi_{30}}}^{\mathcal{P}^{\mu \nu}} \frac{p^{2}}{2}}^{\equiv \Pi_{30}^{\mu \nu}} W_{\mu}^{3} B_{\nu}+ \\
& +\frac{\frac{\left\langle S_{h}^{2}\right\rangle}{4} \Pi_{1}(0)}{\Pi_{0}^{\prime}(0)+\frac{\left\langle S_{h}^{2}\right\rangle}{4} \Pi_{1}^{\prime}(0)}\left\{2 W_{\mu}^{+} W_{\nu}^{-}+W_{\mu}^{3} W_{\nu}^{3}+B_{\mu} B_{\nu}-2 W_{\mu}^{3} B_{\nu}\right\}
\end{aligned}
$$

Provided that the S-parameter breaking term $\widetilde{\Pi_{30}}$ is small, we can rotate by the usual Weinberg angle, and we write the lagrangian in terms of the mass eigenstates.

$$
\begin{aligned}
\mathcal{L}_{E f f, V}^{\mathcal{G}}= & \frac{1}{4} A_{\mu \nu}^{i} A^{i \mu \nu}+\frac{1}{4} B_{\mu \nu} B^{\mu \nu}+{\widetilde{\Pi_{30}}}^{\mu \nu} W_{\mu}^{3} B_{\nu}+ \\
+ & \frac{1}{2}\left(\frac{1}{f^{2} g^{2}} \frac{\Pi_{1}(0)}{\Pi_{0}^{\prime}(0)+\frac{\left\langle S_{h}^{2}\right\rangle}{4} \Pi_{1}^{\prime}(0)}\right) \times \\
& \times\left\{2\left(\frac{v^{2} g^{2}}{4}\right) W_{\mu}^{+} W^{-\mu}+\left(\frac{v^{2}\left(g^{2}+g^{\prime 2}\right)}{4}\right) Z_{\mu} Z^{\mu}\right\} \\
\mathcal{L}_{E f f, V}^{\mathcal{G}}= & \frac{1}{4} A_{\mu \nu}^{i} A^{i \mu \nu}+\frac{1}{4} B_{\mu \nu} B^{\mu \nu}+\widetilde{\Pi_{30}}{ }^{\mu \nu} W_{\mu}^{3} B_{\nu}+ \\
& +m_{W}^{2} W_{\mu}^{+} W^{-\mu}+m_{Z}^{2} Z_{\mu} Z^{\mu}
\end{aligned}
$$


where we have defined

$$
\begin{aligned}
& {\widetilde{\Pi_{30}}}^{\mu \nu} \equiv \mathcal{P}^{\mu \nu} \frac{p^{2}}{2}\left\{\frac{-\frac{g^{2}}{4}\left(\frac{v^{2}}{m_{\rho}^{2}}+\frac{v^{2}}{m_{a}^{2}}\right)}{1+\frac{1}{2} \frac{m_{a}^{2}}{m_{\rho}^{2}} \frac{g^{2} f^{2}}{m_{a}^{2}-m_{\rho}^{2}}-\frac{g^{2}}{4}\left(\frac{v^{2}}{m_{\rho}^{2}}+\frac{v^{2}}{m_{a}^{2}}\right)}\right\} \\
& m_{W}^{2} \equiv \frac{v^{2} f^{2}}{4} \frac{1}{1+\frac{1}{2} \frac{m_{a}^{2}}{m_{\rho}^{2}} \frac{g^{2} f^{2}}{m_{a}^{2}-m_{\rho}^{2}}-\frac{g^{2}}{4}\left(\frac{v^{2}}{m_{\rho}^{2}}+\frac{v^{2}}{m_{a}^{2}}\right)} \\
& m_{Z}^{2}=\frac{m_{W}^{2}}{c_{W}^{2}}
\end{aligned}
$$

From the lagrangian, we can see that there will be a contribution to the PeskinTakeuchi $S$ parameter [12]. These precision parameters are defined as:

$$
\begin{aligned}
& \alpha T=\frac{\Pi_{W W}(0)-\Pi_{33}(0)}{m_{W}^{2}}=0 \\
& \alpha S=-4 s_{w} c_{w} \Pi_{30}^{\prime}(0) \\
& \alpha U=4 s_{w}^{2}\left(\Pi_{W W}^{\prime}(0)-\Pi_{33}^{\prime}(0)\right)=0
\end{aligned}
$$

The t-parameter being zero at tree level is a direct consequence of the imposition of custodial symmetry in the $\mathrm{MCHM}_{5}$. The S-parameter is given by

$$
\alpha S=-\frac{g g^{\prime}}{g^{2}+g^{\prime 2}}\left(\frac{v^{2}}{m_{\rho}^{2}}+\frac{v^{2}}{m_{a}^{2}}\right)
$$

So at the end, we have that $\alpha S \sim \mathcal{O}\left(v^{2} / f^{2}\right)$. The study of precision tests in CHM was extensively done in the literature $[30,29,23]$. It turns out that in a nonrenormalizable theory such as this one, the precision parameters get logarithmic contributions that are divergent. So, the study of these kinds of bounds is very constraining in CHMs. In chapter 5, we will briefly discuss some of these bounds. 


\subsection{Overview of the Form Factor Formalism}

Compositeness signals are accessed through the analytical properties of the correlation functions. This is the crucial point in the form factor formalism, which introduces functions of the momentum in the Higgs interactions. These expressions reflect the underlying dynamics of the strong sector.

We provided some simple models in which the form factor could be developed. Even though these ansatzes stand as models of their own, they served as a warmup of the more concrete realization of the form factor formalism in CHMs. As an example, the MCHM5 was sufficient to illustrate how dynamical deviations from the usual zero momentum limit appear as a consequence of resonant behaviour.

Traditionally in CHM literature, the form factor parametrization is restricted to the analysis of a non-dynamical Higgs. Such approximation limits the full momentum dependence to just $p^{2}$. As discussed, in this case, the phenomenological possibilities get limited to the physical mass running of the Fermions and Gauge bosons.

More notably, the most critical effects appear when including the full structure of three-point functions. In such interactions, the recovery of the fully dynamical Higgs and the possibility of off-shellness provides an excellent opportunity to study new phenomenological effects. Additionally, the EFT analysis showed that the dynamical effects could be competing to the misalignment suppression at zero momentum.

Furthermore, since the modification is dynamical, the shape of distribution functions should be altered beyond an overall normalization. This signals the phenomenological effects due to compositeness that we have been looking for. Now, after restoring the full momentum dependence of the form factor formulation, we can explore the enriched phenomenology it grants. 


\section{Chapter 5}

\section{Collider Phenomenology of Form}

\section{Factors}

Throughout this work, we developed the notion that the Higgs has an internal structure. A notion that leads us to the study of form factors and the necessity of exploring the momentum dependence in the Higgs interactions experimentally. From the experimental point of view, it remains - What are the signs that the Higgs is a composite particle?

The program now is to reduce the conceptual picture of elementarity versus compositeness to the empirical study of the Higgs interactions. Fortunately, the experimental effort today is aligned with this goal as the LHC next stages are devoted to Higgs analysis. We are starting to access the Higgs interactions, and with it, capable of checking if there are departures from the SM.

In the case of a composite Higgs, the immediate effects are the shifts in the couplings that appear as misaligned suppressions. There, it occurred a modification due to vacuum misalignment, a consequence of the strong sector. We denoted these as the zero momentum limit of form factors. In them, the dynamically generated effects are not accessed when the momentum transfer is low.

However, not too far above energetically-wise, we should start to see form factors effects. As implied before, the most critical ingredient to access the form factors is by going of-shell. Thus, the importance of having off-shell processes cannot 
be stressed enough, since these will allow us to explore the different regions in the form factor momentum plane.

If such scenario became real, the next step would be to undergo a process similar to the partonic studies that unraveled the structure of Hadrons. Eventually, the core constituents responsible for confining the Higgs would become evident, providing hints at a complete form of the SM.

However, much before this picture takes place, it is essential to have a clear way to study compositeness signs. For that, the first step is the implementation of form factors and predicting how visible these effects would be. It goes down to find the most sensible experimental observables and channels to the LHC in its upcoming stages, High Luminosity (HL) and High Energy (HE).

First, the Higgs is already reasonably well characterized by currents experiments. Earlier in 2012, the Higgs mass and basic properties were determined at the discovery [7, 8]. Now, the mass is known below the per cent level, $m_{h}=125.09 \pm 0.3$ $\mathrm{GeV}$. Subsequently, at the second run with $\sqrt{s}=13 \mathrm{TeV}$, the LHC mostly confirmed the SM predictions, even though with not great precision [9]. In the HL phase, we expect to improve these measurements, tackling naturalness to its core.

Currently, many Higgs productions channels were explored to some degree [10, 90, 92, 93, 96, 97]. The cross sections for the various processes are:

- Gluon Fusion $\sigma(g g \rightarrow h) \sim 44 \mathrm{pb}$.

- Vector Boson Fusion $\sigma(p p \rightarrow q q h) \sim 4$ pb.

- Higgs-strahlung with W's $\sigma(p p \rightarrow W h) \sim 1.5 \mathrm{pb}$.

- Higgs-strahlung with Z's $\sigma(p p \rightarrow Z h) \sim 0.88 \mathrm{pb}$.

- Associated production with top quarks $\sigma(p p \rightarrow t \bar{t} h) \sim 0.5 \mathrm{pb}$.

- Double Higgs production $\sigma(p p \rightarrow h h) \sim 0.02 \mathrm{pb}$.

The most recent results, from LHC's run 2 are displayed in figure 5.1. 


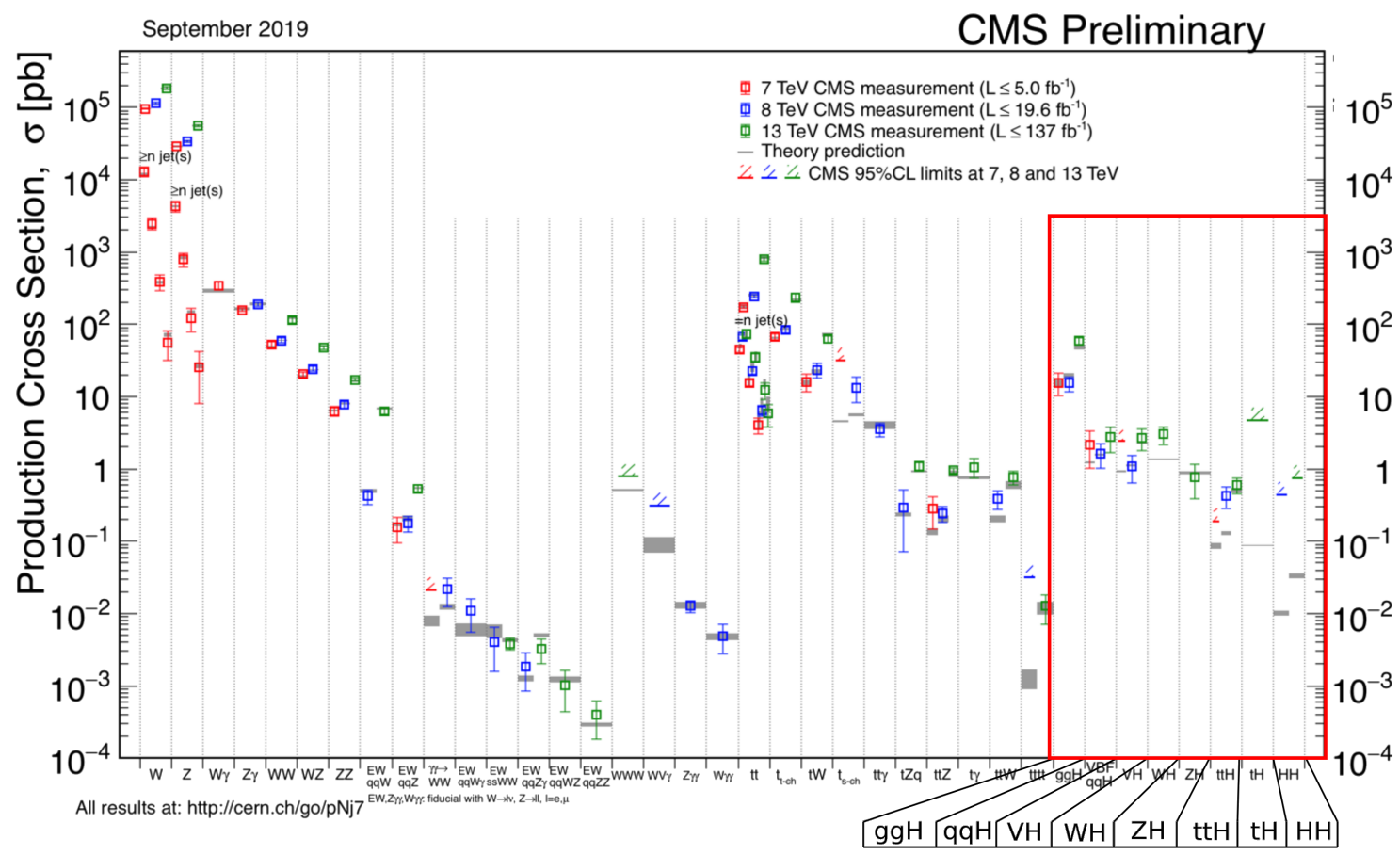

FiguRE 5.1: Summary of the cross section measurements of SM processes at the LHC. We have highlighted the cross-sections involving Higgs processes in the red box.

Additionally, the Higgs decays are summarized in table 5.1. To extract the most promising signals, we shall look for the optimal combination of production mechanism and decay mode.

\begin{tabular}{ll}
\hline Decay Channel & Branching Ratio [\%] \\
\hline$h \rightarrow b b$ & $57.5 \pm 1.9$ \\
$h \rightarrow W W$ & $21.6 \pm 0.9$ \\
$h \rightarrow g g$ & $8.56 \pm 0.86$ \\
$h \rightarrow \tau \tau$ & $6.30 \pm 0.36$ \\
$h \rightarrow c c$ & $2.90 \pm 0.35$ \\
$h \rightarrow Z Z$ & $2.67 \pm 0.11$ \\
$h \rightarrow \gamma \gamma$ & $0.228 \pm 0.011$ \\
$h \rightarrow Z \gamma$ & $0.155 \pm 0.014$ \\
$h \rightarrow \mu \mu$ & $0.022 \pm 0.001$ \\
\hline
\end{tabular}

TABLE 5.1: Higgs Branching Ratios. Data from [90].

Each one of these channels has their particularities, and determining the most efficient one to probe the Higgs is not just about cross-section visibility. The reach 


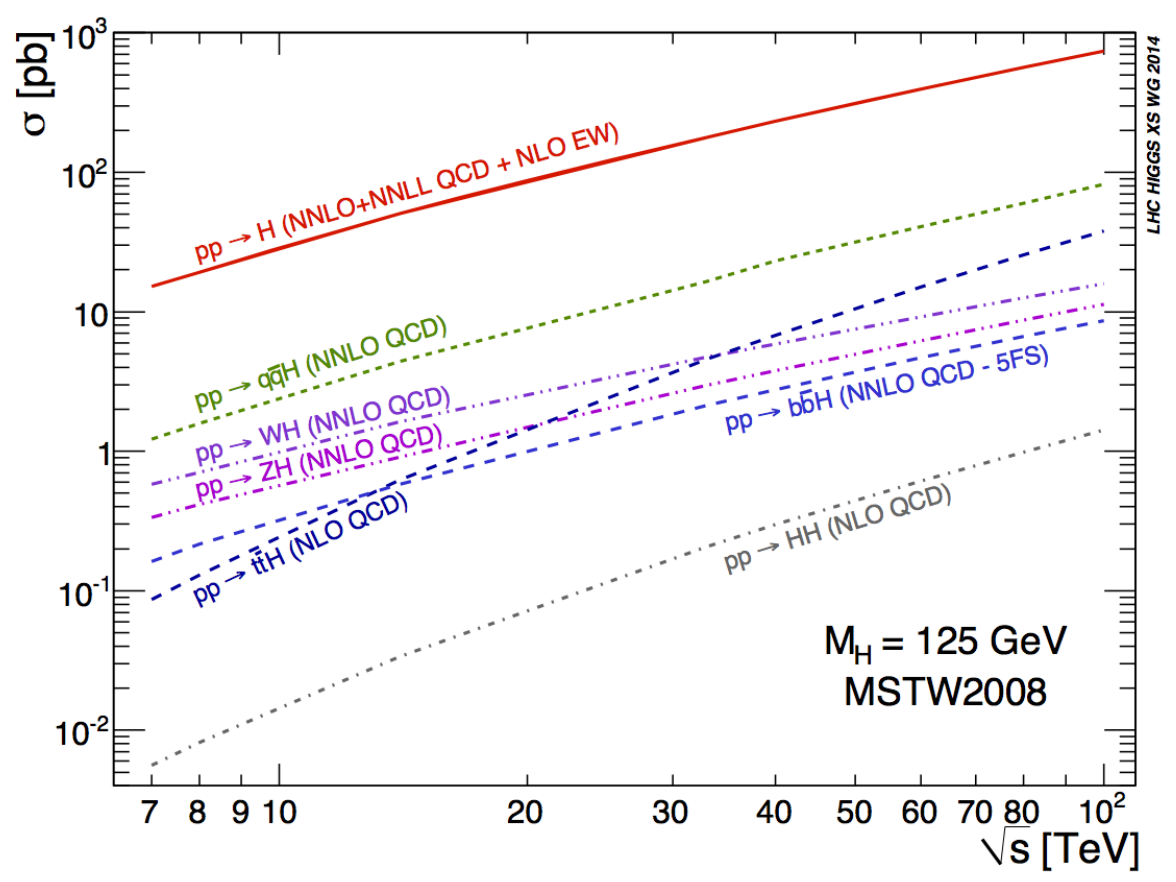

FiguRE 5.2: Simulations of proton-proton cross-sections for future $\sqrt{s}$.

of each channel is determined by a signal-background analysis, which is crucial to see the full discovery potential of the given process.

Further into this chapter, we will select the most promising channels for form factor studies. As anticipated, the most distinctive ones will have top quarks or gauge bosons far off-shell. Also, having the Higgs off-shell is interesting mainly in models in which the form factor couples with thee Higgs momenta, as in the simple ansatz of the heavy scalar mixing case.

Experimentally, every observable is constructed from the four-vectors that define the final state of the collision. From these, one can build all the distribution functions. Ultimately, these distributions will reflect all the kinematical properties of the collision.

Often, the off-shell tails of distribution functions are the best indicative of modifications to the internal structure. 


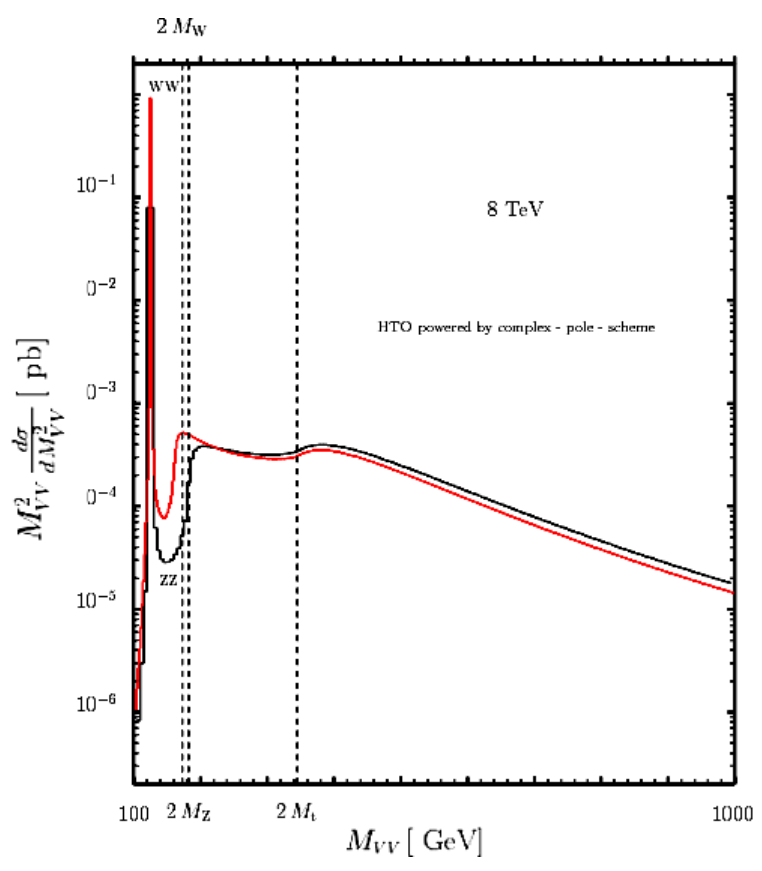

Figure 5.3: Simulation of the invariant mass distribution of $g g \rightarrow V V$ process. Figure extracted from [91]. Such process involves the analysis of an off-shell Higgs as an intermediate state. The distribution tail after the $2 M_{t}$ mark is the most sensitive region for offshell effects.
As an example of an off-shell analysis, consider the process $g g \rightarrow V V$. This has an off-shell Higgs contribution $g g \rightarrow h^{*} \rightarrow V V$ leading to an invariant mass distribution as in figure 5.3. One can see the peak at the resonance - the Higgs becoming on-shell at $125 \mathrm{GeV}$.

Distinguishly, after the mark of $2 M_{Z}$ the distribution tail receives the off-shell contributions from the off-shell Higgs and other diagrams. This tail is the section that is most sensitive to form factor effects $[38,39]$ and is the target of our work. Finding modifications of the distribution functions such as this one is the most important sign of a composite Higgs.

\subsection{Zero Momentum Phenomenology of CHMs}

The zero momentum is the first-order approximation of form factor effects and should provide constraints to the parameter space. There, we must explore a set of parameters that are not yet ruled out by current data from previous studies.

In the CHMs, the coupling constants were modified by functions of $\xi=v^{2} / f^{2}$ because of vacuum misalignment. Keeping $\mathcal{O}(\xi)$ effects while disposing of $\mathcal{O}\left(p^{2} / m_{*}^{2}\right)$ from form factor dynamics implies a further decoupling of dynamical effects from resonances. The dynamical vertices effects are reduced to the misalignment suppression when the scale of the resonances $m_{*}$ is far from $f$, mainly when the couplings $g_{*} \sim 4 \pi$. 
When there is a complete decoupling, we expect non-dynamical effects and the recovery of usual CHM phenomenology.

$\mathrm{CH}$ phenomenology was mainly studied with indirect and direct signals. The indirect is composed of results from Electroweak precision tests and SM-couplings modifications in Atlas and CMS at the LHC. Alternatively, Direct searches are related to the direct production of resonances, with the calculation of production and decay rates.

Indirect searches are aimed to constrain the scale of the new sector $f$ through modifications of the Higgs couplings. In figure 5.4 we can see the bounds due to the $\mathrm{S}$ and $\mathrm{T}$ parameters from precision tests, and figure 5.5 shows the LHC couplings modifiers. Loosely, we should adopt $\xi \leq 0.1$, which gives a new physics scale of $f=800 \mathrm{GeV}[28]$.

The direct searches involve the completion of the theory with some part of the resonance spectrum. In these searches, the scalar, fermion and vector partners are explicitly produced in physical processes. The decay rates are calculated, and the signals in colliders get identified, moving the bounds of the masses of the resonances as data continues to improve.

In figure 5.6, we can see the current and projected bounds on the collider searches for vector partners due to the strongly interacting sector. A value to keep as a reference is $m_{*} \sim 800 \mathrm{GeV}$, below that is hard to keep up with experimental bounds. 


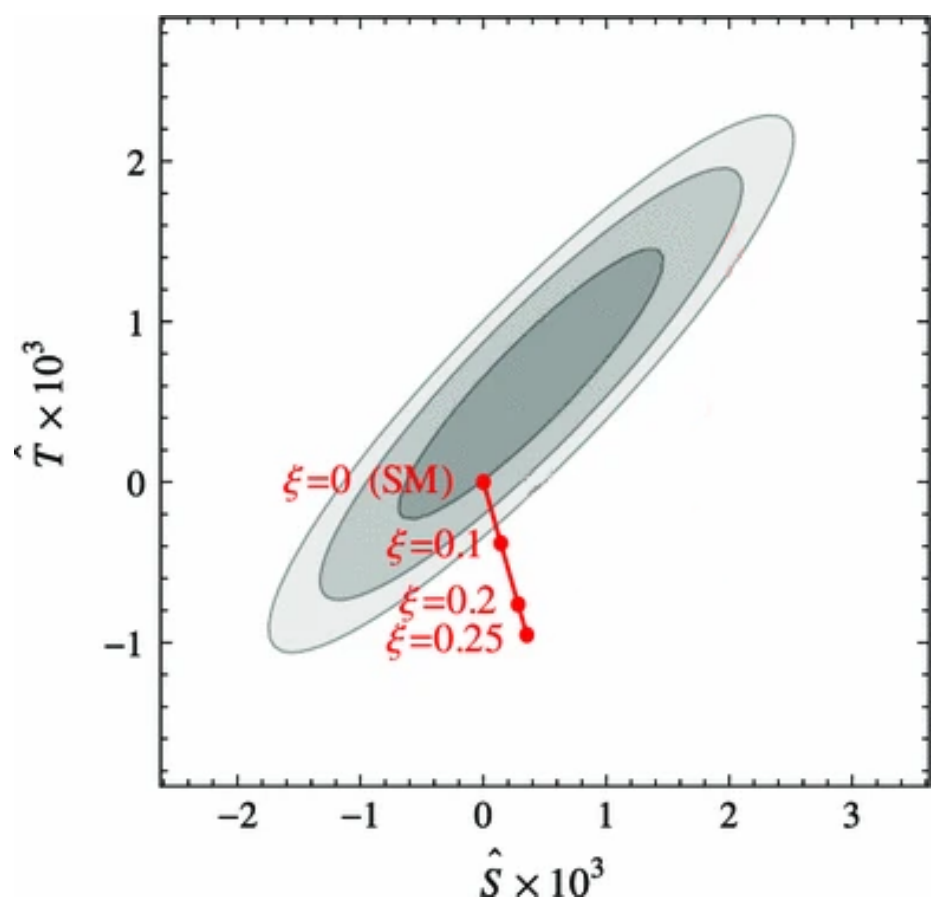

Figure 5.4: Confidence level contours of $68 \%, 95 \%$ and $99 \%$ on the oblique EW $\hat{S}$ and $\hat{T}$ parameters. The red line indicates the MCHM5 predictions. Figure taken from [29].

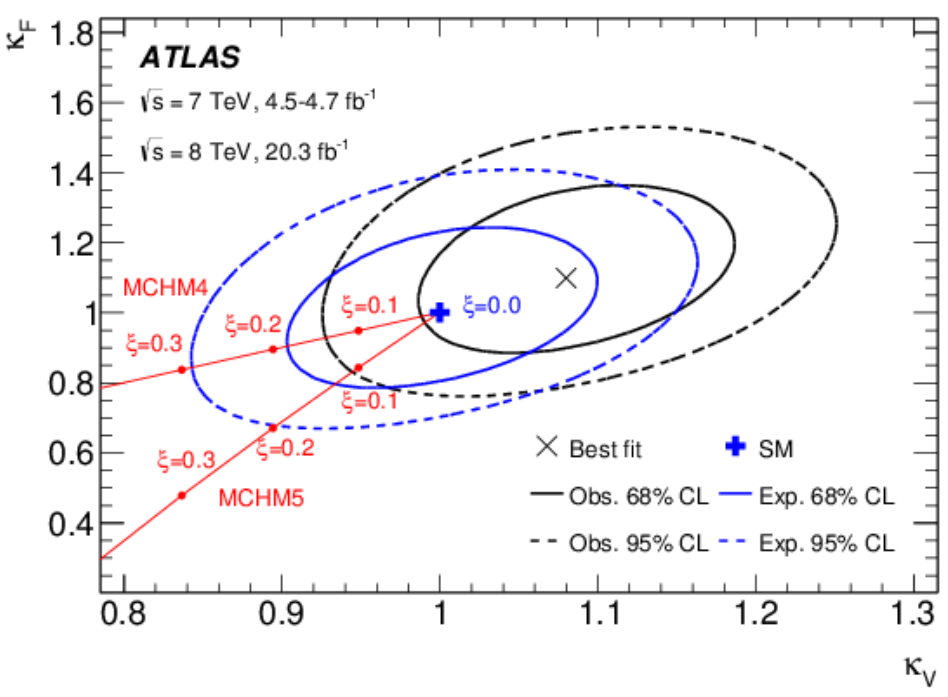

Figure 5.5: Confidence level contours on the SM interaction modifiers $\left[\kappa_{V}, \kappa_{F}\right],[36]$. The red curves indicate the predictions for the MCHM4 and MCHM5 models, compared to the SM expectation and experimental fit. 

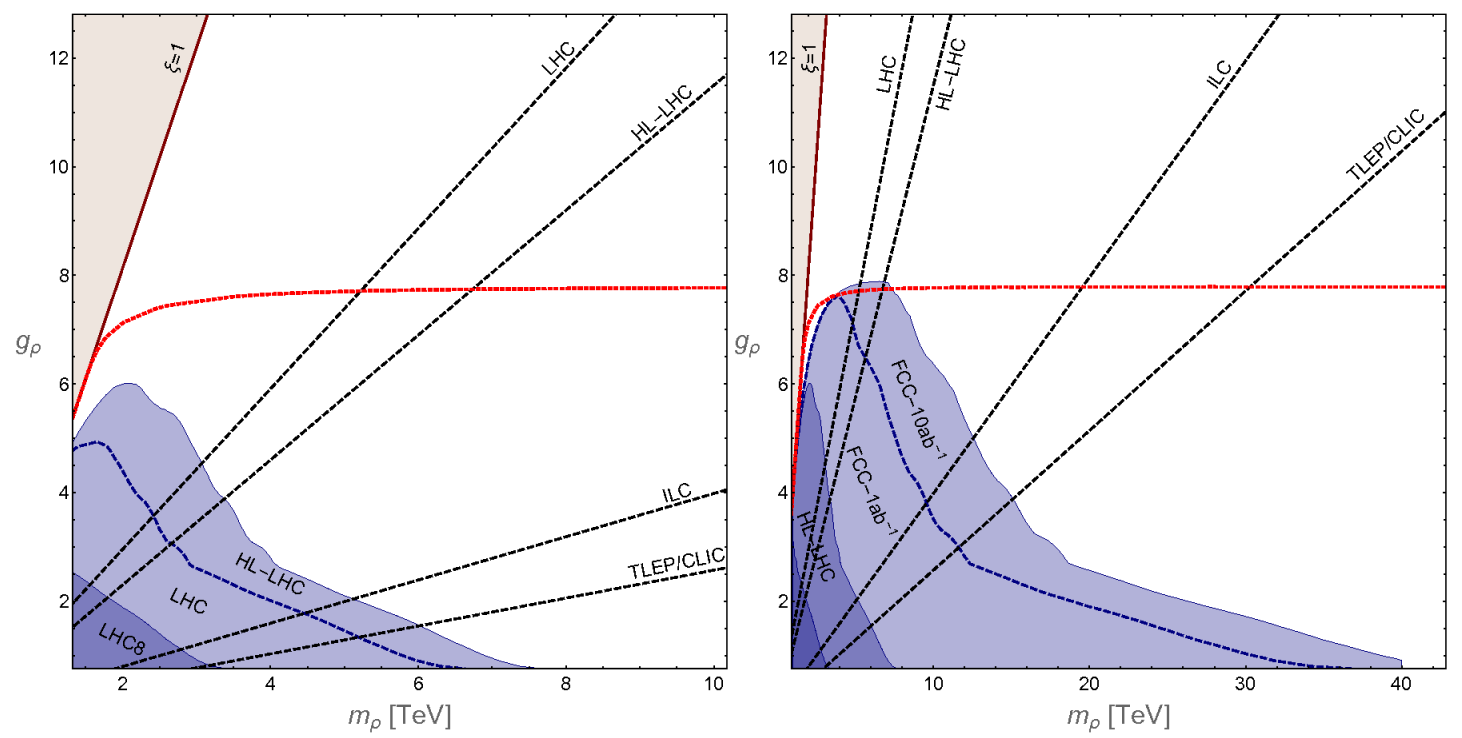

FiguRE 5.6: Direct searches bounds on $\left(m_{\rho}, g_{\rho}\right)$. Figure taken from [28]. The exclusion regions for collider data are highlighted in blue. Depending on the value of the coupling, the LHC can set up a bound of up to $m_{\rho}=8 \mathrm{TeV}$, which will be increased to $m_{\rho}=10 \mathrm{TeV}$ in the HL phase (left panel). The right figure indicate the predictions for future experiments.

\subsection{Form Factor Phenomenology}

From the numerical point of view, the inclusion of form factors vertices is not a standard procedure in usual Monte Carlo (MC) approaches to collider physics. As of the methods used, we shall describe the implementation and simulation of the signal at the parton level.

In our study, the analysis was limited to parton level, without the detector simulation and inclusion hadronization effects. These last will be a subject for future research. However, the main contributions we seek are already visible at the parton level. The critical point is that through this initial study, we achieve concrete signals of what a composite Higgs looks like experimentally. 


\subsubsection{Numerical Implementation}

In numerical applications of particle physics, Monte Carlo methods are broadly used to simulate the collision process. MC is very analogous to a direct simulation of the physical process. In it, the quantities of interest (i.e. cross sections and decay rates) are integrals of weight functions over variables of physical significance.

The method of integration consists of generating a representative sample and averaging over the integrand. The representative set is a real random sample is generated and averaged over the given weights. Physically, the amplitudes are integrated from phase space and continuum variables, like incident partons and fragmentation products.

MadGraph [102] is a software that aims to the computation of physical observables using MC methods. It generates a process and uses tools for manipulation and analysis of the hard events simulations. For tree-level calculations, the input is an user-defined "Lagrangian" packed into a file. This file includes all the interactions of the model.

Such Lagrangians are usually expressed through the Mathematica based, FeynRules [99] and get translated into the standard Universal FeynRules Output (UFO) format [98]. The UFO file uses a Phyton base syntax, called ALOHA [103], in order to write the vertices and Lorentz structures of the theory. These get further translated to Madgraph by another format, the HELAS output, that is Fortran based and more adequate to the internal structure of event generation procedure.

The inner workings are intricate but, in short, the UFO output is the userdefined lagrangian that goes to Madgraph which computes the physical amplitudes. The general structure of a UFO file is:

The generation of UFO files with form factors is not explicitly done within the FeynRules framework. So, in order to achieve our goal, it was necessary to modify the internal structure of the UFO model.

The necessary alteration is to include a general dependence on the momentum to the vertices of the model. The easiest way to do this is by defining the form 
factor in the UFO model using an ALOHA syntax. ALOHA then translates the vertices functions to a FORTRAN output, that is directly used to perform the matrix elements calculations.

For example, the inclusion of the top Yukawa form factor is done as follows.

First, the vertices were altered in the file vertices.py of the default sm UFO file.

vertices.py

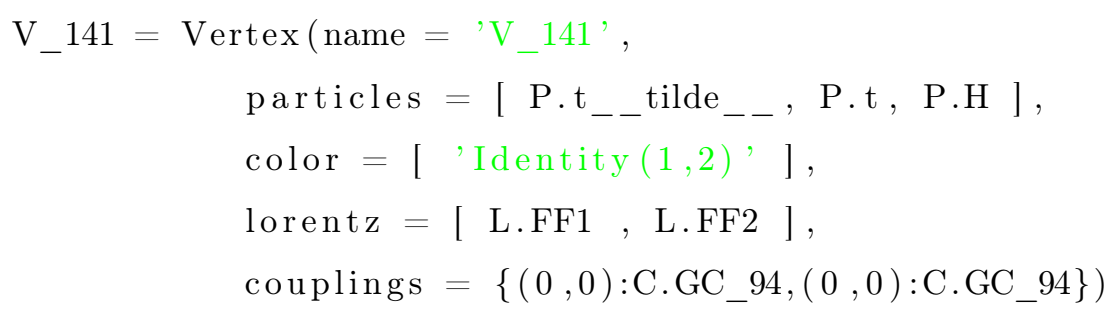

Then, this vertex call for the defined Lorentz structures FF1 and FF2, located in the lorentz.py file.

lorentz.py

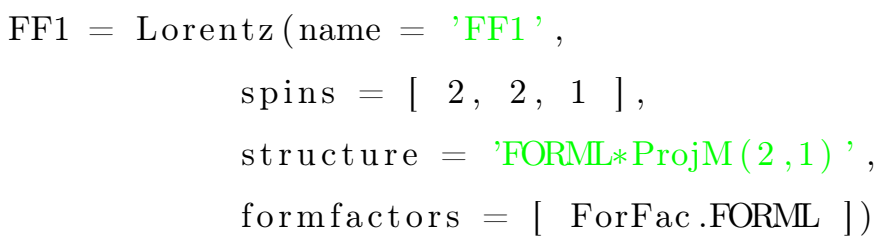

$\mathrm{FF} 2=\operatorname{Lorentz}\left(\right.$ name $={ }^{\prime} \mathrm{FF} 2{ }^{\prime}$,

$\operatorname{spins}=[2,2,1]$,

structure $=$ 'FORMR $* \operatorname{ProjP}(2,1) \sqsupset$ ',

formfactors $=[$ ForFac.FORMR ])

Finally the form factor expressions for FORML and FORMR are defined in the formfactors.py file and the additional parameters of the theory in parameters.py.

formfactors.py 


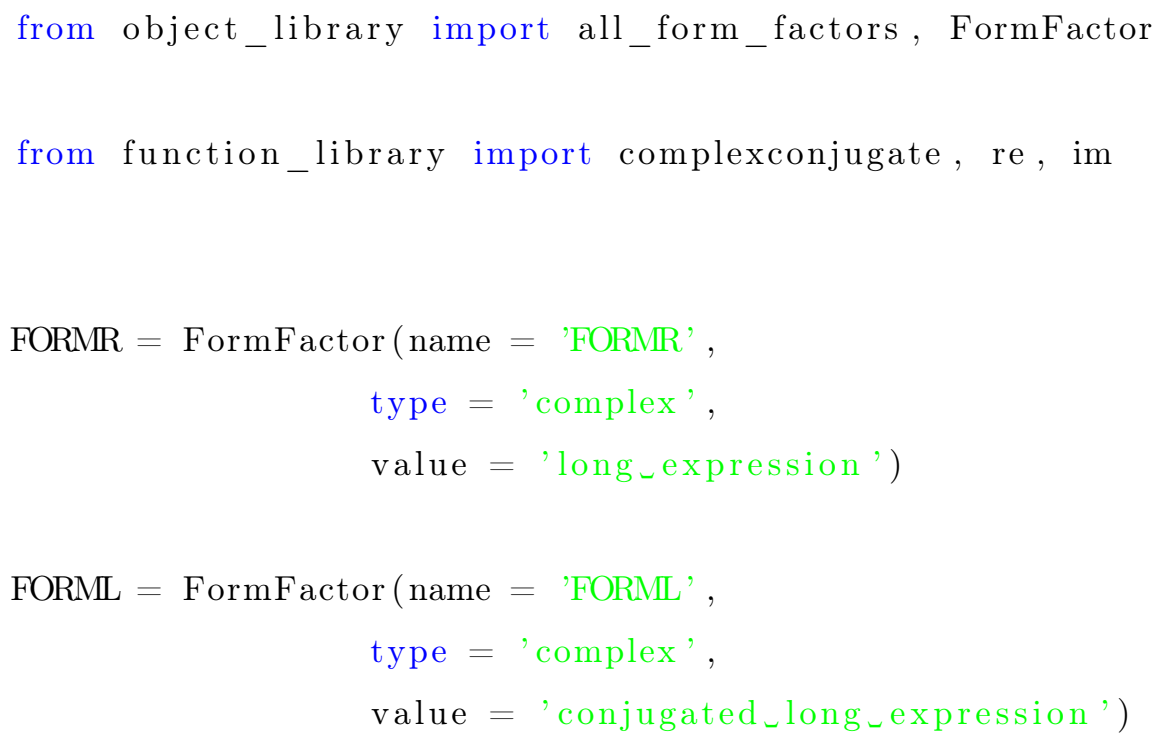

The output in Helas format corresponds to the following Yukawa FFS vertex

$$
\begin{aligned}
\mathcal{L}_{F F S} & =\bar{f}^{\prime}\left(G C(1) \frac{1-\gamma_{5}}{2}+G C(2) \frac{1+\gamma_{5}}{2}\right) f S \\
& =\text { FORML } * \bar{t}_{r} t_{l} h+\text { FORMR } * \bar{t}_{l} t_{r} h
\end{aligned}
$$

Once the form factor model is defined, the generation of events can be carried through in the usual manner.

After discussing the implementation, we return to the task of exploring the Higgs production channels that amplify the effects of form factors. We shall highlight the tth, Zh and gluon fusion because of their essential properties concerning the momentum flow and experimental reach. 


\subsubsection{The tth Channel}

Starting with a proton-proton collision, the tth features the production of a Higgs and two top quarks. Diagramatically, the process is represented by:

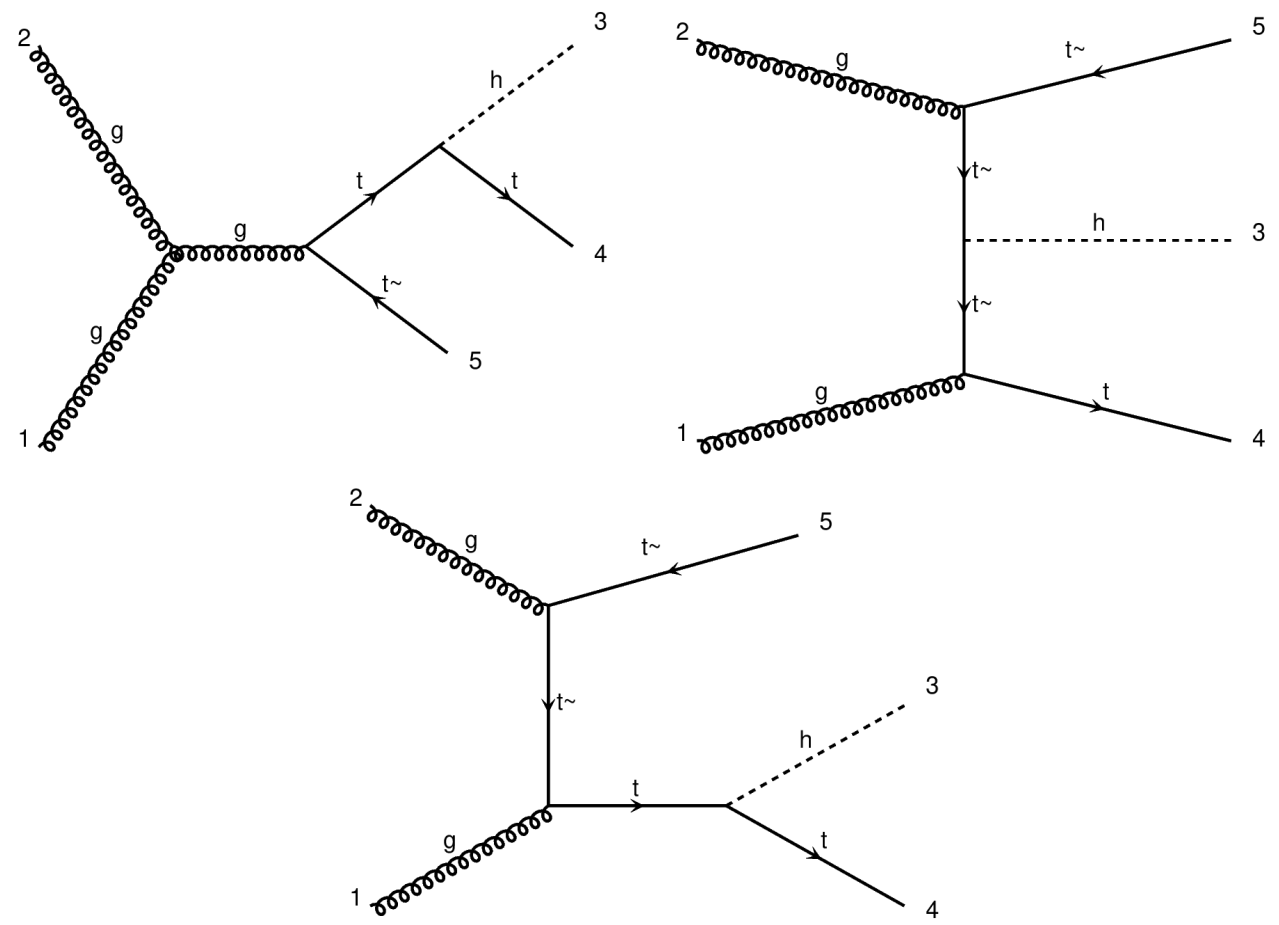

FiguRE 5.7: Representative diagrams of the th channel.

Notably, the tth channel possesses much background with the two tops decaying hadronically or semi-leptonically. The options for the Higgs decays also add to the complexity of the problem since it preferably will decay to two b-quarks, leading to more jets.

Even though tth is a tricky channel for Higgs production, it is the best probe to the Yukawa form factor. In it, we are accessing the interaction of the Higgs with the top quarks directly. tth has a significant contribution of an intermediate off-shell Top-quark, that is the requirement for form factor effects. Furthermore, prospects become better as $\sqrt{s}$ increases since th will become more significant as figure (5.2) suggests. 
When a top quark "strahlungs" to produce a Higgs and another top, it must be highly off-shell, with its momentum much above the physical mass region. Because of this property, we can access the form factor, and its effects appear in the observables.

Remember that the form factor was obtained in chapter 4, and is given by:

$$
\begin{aligned}
y_{t}(p, q) & =\frac{1}{\sqrt{2}} \frac{M(p, q)(1-2 \xi)}{\sqrt{\Pi_{0}^{L}(p)+\Pi_{1}^{L}(p) \frac{1}{2}\left\langle S_{h}^{2}\right\rangle} \sqrt{\Pi_{0}^{R}(q)+\Pi_{1}^{R}(q)\left\langle C_{h}^{2}\right\rangle}} \\
& =\frac{\frac{(1-2 \xi)}{\sqrt{2}} y_{l} y_{r} e^{i\left(\phi_{l}-\phi_{r}\right)}\left[\frac{m_{4}}{p^{2}-m_{4}^{2}}-\frac{m_{1}}{q^{2}-m_{1}^{2}}\right]}{\sqrt{1+\frac{f^{2} l_{l}^{2}}{p^{2}-m_{4}^{2}}+\frac{v^{2} y_{l}^{2}}{2}\left(\frac{1}{p^{2}-m_{1}^{2}}-\frac{1}{p^{2}-m_{4}^{2}}\right)} \sqrt{1+\frac{f^{2} y_{r}^{2}}{q^{2}-m_{1}^{2}}+y_{r}^{2}\left(\frac{f^{2}-v^{2}}{q^{2}-m_{1}^{2}}-\frac{f^{2}-v^{2}}{q^{2}-m_{4}^{2}}\right)}}
\end{aligned}
$$

Here the widths are not explicitly written, but are implied in the pole. Also, we have redefined the complex $y$ couplings such as they are real. In this way, there are 8 parameters in the form factor expression: $f, m_{1}, m_{4}, \Gamma_{1}, \Gamma_{4}, y_{l}, y_{r}$ and $e^{i\left(\phi_{l}-\phi_{r}\right)}$. In order to constrain the parameter space, we shall impose some physical requirements to the expression.

\section{Theory Constraints}

There are two major constraints for the tth vertex.

First, the parameters must be such that we can obtain a light Higgs and that its mass can be fixed to $125 \mathrm{GeV}$. In chapter 2 we enunciated the condition for the obtainment of a light Higgs from the Coleman-Weinberg potentail. From [25], the Higgs mass is estimated to be

$$
\begin{aligned}
& m_{h}^{2}=\frac{N}{\pi^{2}}\left[\frac{m_{t}^{2}\left(m_{1}^{2} m_{4}^{2}\right) \log \left(\frac{m_{1}^{2}}{m_{4}^{2}}\right)}{f^{2}\left(m_{1}^{2}-m_{4}^{2}\right)}+\frac{v^{2}}{4}\left(1-\frac{v^{2}}{f^{2}}\right)\left(\Delta y^{2}\right)^{2}\left(\frac{\left(m_{1}^{2}+m_{4}^{2}\right) \log \left(\frac{m_{1}^{2}}{m_{4}^{2}}\right)}{2\left(m_{1}^{2}-m_{4}^{2}\right)}-1\right)\right] \\
& \Delta y^{2} \equiv\left|y_{l}\right|^{2}-2\left|y_{r}\right|^{2}
\end{aligned}
$$

From (5.4), we can see that if $\Delta y^{2}$ is small, the Higgs mass is practically fixed by the relation between $m_{1}$ and $m_{4}$. This process is illustrated in figure 5.8. 
For now, we will limit to small $\Delta y^{2}$. Keeping $\Delta y^{2}<1$ allow us to satisfy the Higgs mass without having to specify the values of $y_{l}$ and $y_{r}$, so at the end we write $m_{1}$ as a function of only $m_{4}$.

Now for the other constraint, we have to respect the top mass-Yukawa relation in the $\mathrm{MCHM}_{5}$. By going to zero momentum in the form factor, the following relation must be satisfied.

$$
y_{t}=k_{5} \frac{\sqrt{2} m_{t}}{v}=\frac{1-2 \xi}{\sqrt{1-\xi}} \frac{\sqrt{2} m_{t}}{v}
$$

Using (5.5), we can see what values of $y_{l}$ and $y_{r}$ will satisfy the top mass at $172.4 \mathrm{GeV}$.

From figure (5.9), we see that it is possible to choose multiple values of $y_{l}, y_{r}$ that lead to the correct top mass. Also, some values of the chosen mass ratios $g$ 's do not satisfy the top constraint.

What should happen in this $y_{l}-y_{r}$ plane is that the lower the $y$ 's are, the less the heavy resonances mixture with the elementary fermions. This effect lead to subtler effects on the form factor enhancement. This becomes clear in figure 5.10, in which we plot the form factor as a function of $p$ and $q$ for different insets of $y$ 's.

Choosing a set of $y$ 's and requiring the top mass to be real at zero momentum allows us to fix all parameters. The models can then be imported to MADGRAPH, and we can extract the cross-section as a function of $f$.

\section{Results}

Now that the parameter space is constrained correctly, we can run the simulations and extract the most immediate observable - the cross-section modification.

We choose the mass relationship between the two fermionic resonances to be constant and vary the compositeness scale $f$. Running the collision at $\sqrt{s}=14 T e V$ with $10^{6}$ events generated for each $f$ value, we have the cross-section for the minimal and maximal mixing constants in figure 5.11. 

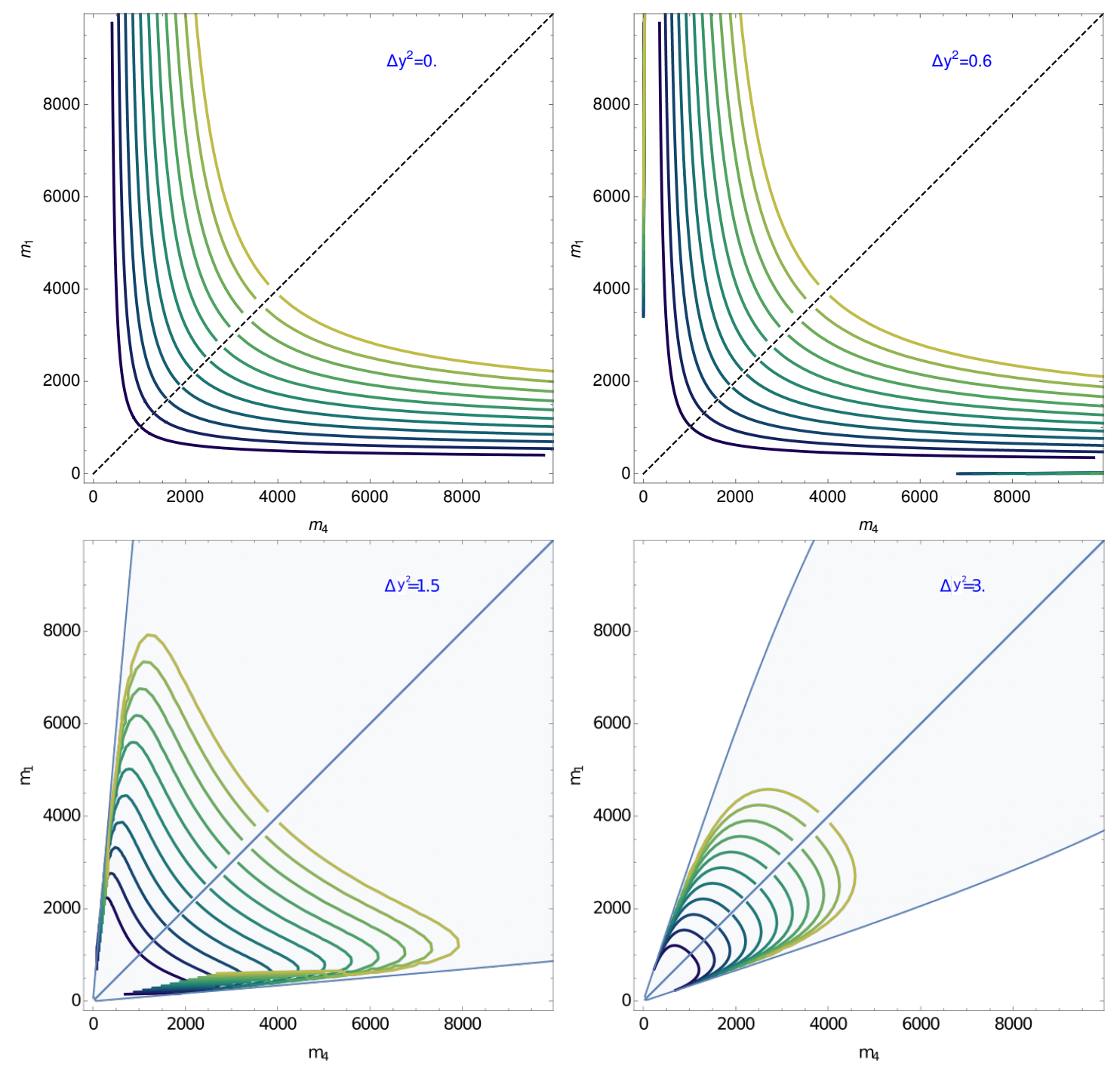

FIGURE 5.8: $m_{1}-m_{4}$ plane scanned by increasing $f$. Each curve correspond to a set of $m_{1}, m_{4}$ that satisfies the Higgs mass at 125 $\mathrm{GeV}$ in (5.4). The darker colours are for the lower f's and it goes from $f=777 \mathrm{GeV}$ to $f=5000 \mathrm{GeV}$. 

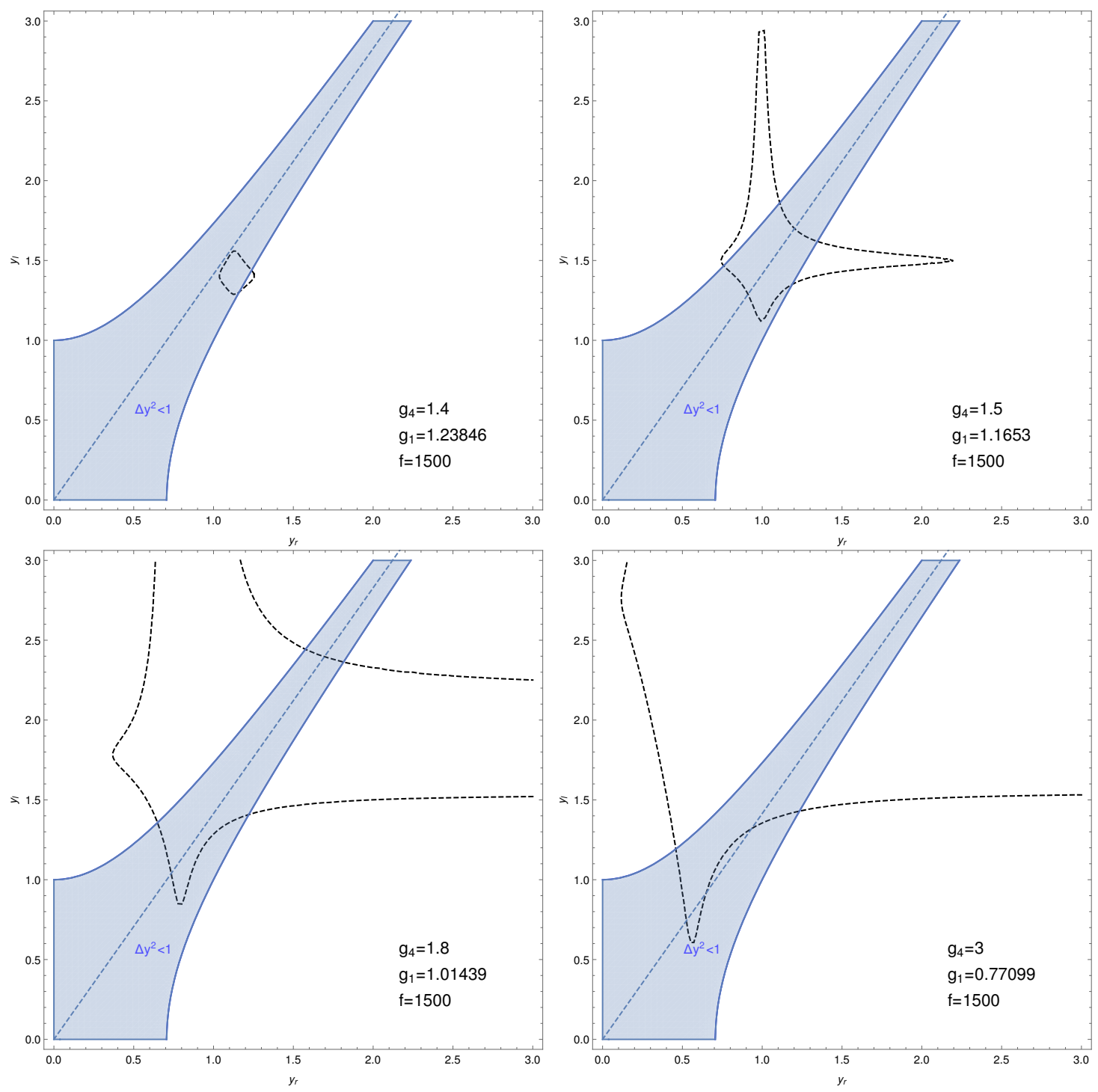

FiguRE 5.9: $y_{l}-y_{r}$ plane. Each point in the dashed contour correspond the set of $y_{l}, y_{r}$ that satisfies the top mass-yukawa relation with $m_{\text {top }} 172.4 \mathrm{GeV}$. 

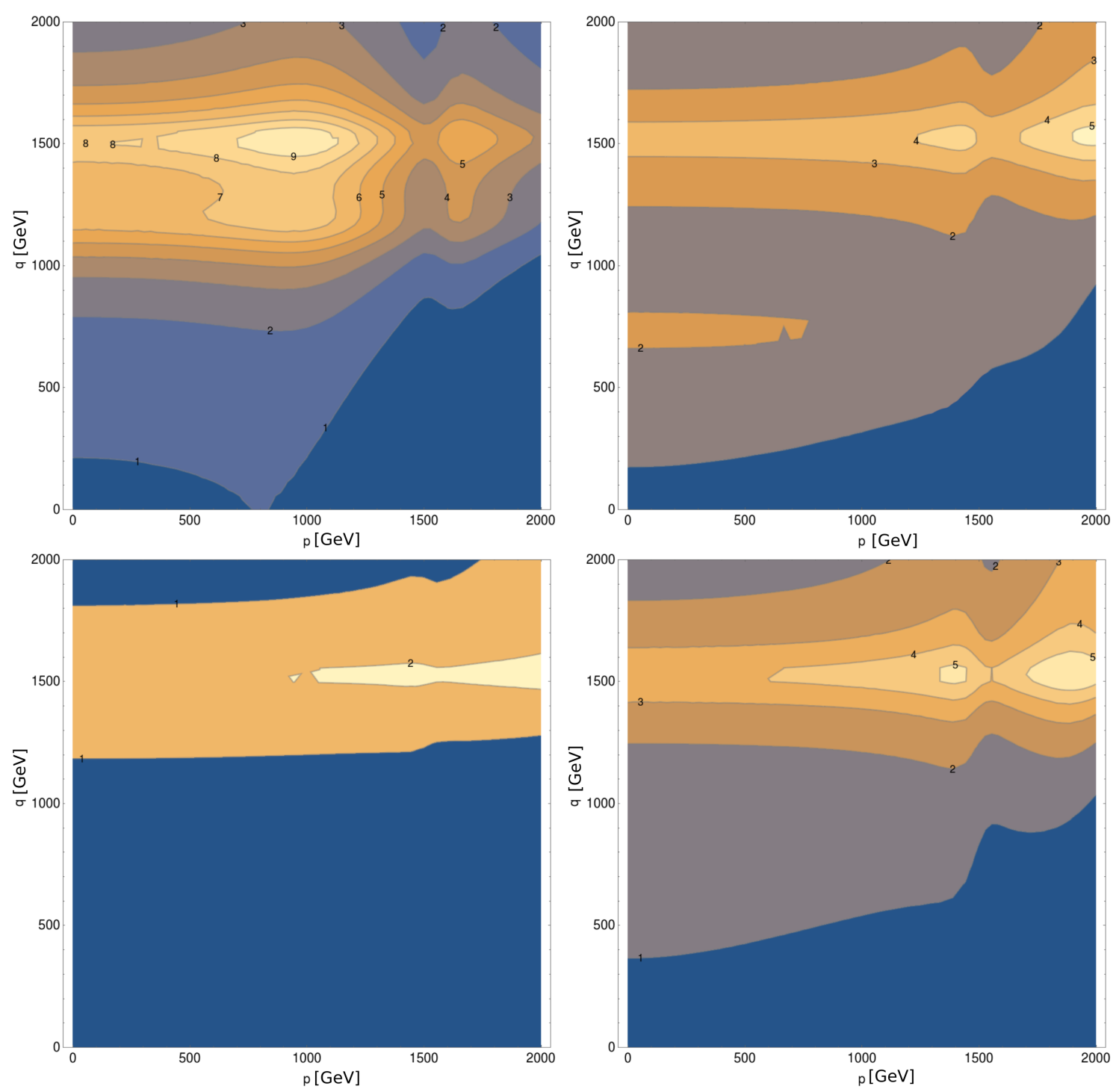

Figure 5.10: Form factor contours in the $p-q$ plane. We show the form factor behaviour as we parametrically walk through the $y_{r}-y_{l}$ plane. We can see that the form factor is well behaved near the origin, where we imposed the mass-Yukawa normalization condition. For low momentum the form factor is the top yukawa value with the $\kappa_{t}^{\mathbf{5}}$ contribution. 


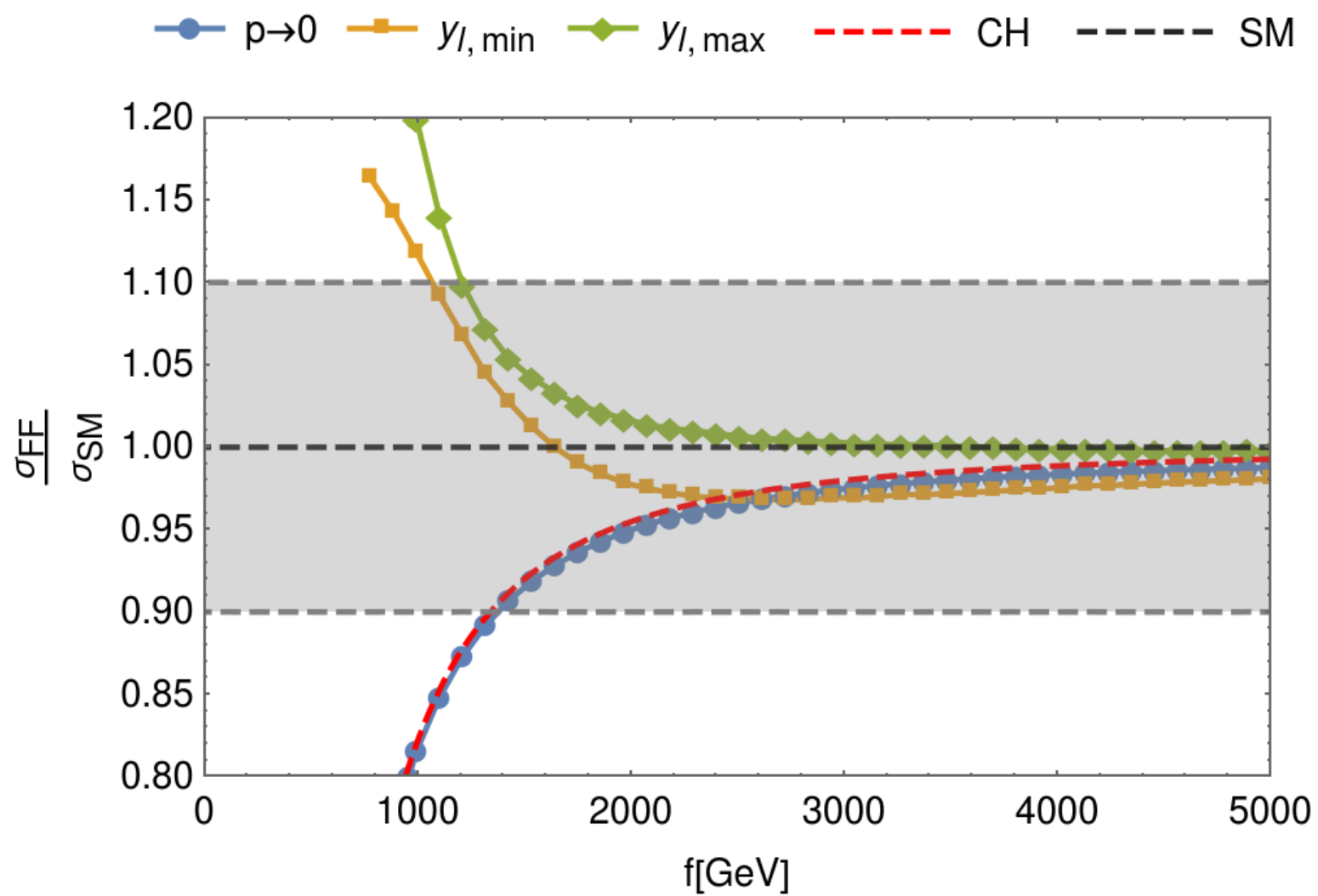

Figure 5.11: Cross section of $t \bar{t} h$ as a function of $f$ for the MCHM5 with form factors. Signal simulation done in Madgraph at partonlevel. The plot is normalized to the SM prediction. CHMs without momentum dependence have an overall suppression of vertices that goes with $\kappa_{5}=(1-2 \xi) / \sqrt{1-\xi}$. Such suppression is depicted in the dashed red curve. By turning on the form factor effects, we get an enhancement due to the analytic structure of their expressions. Fixing all parameters by theory constraints leave us with a minimum or maximum mixing with the resonance, that is controlled by $y_{l}$. These are the yellow and green curves. Finally, $p \rightarrow 0$ is the simulation with form factors but by setting the transferred momentum to zero. Here check that the normalization of the form factors and the zero momentum CHM prediction are the same. 

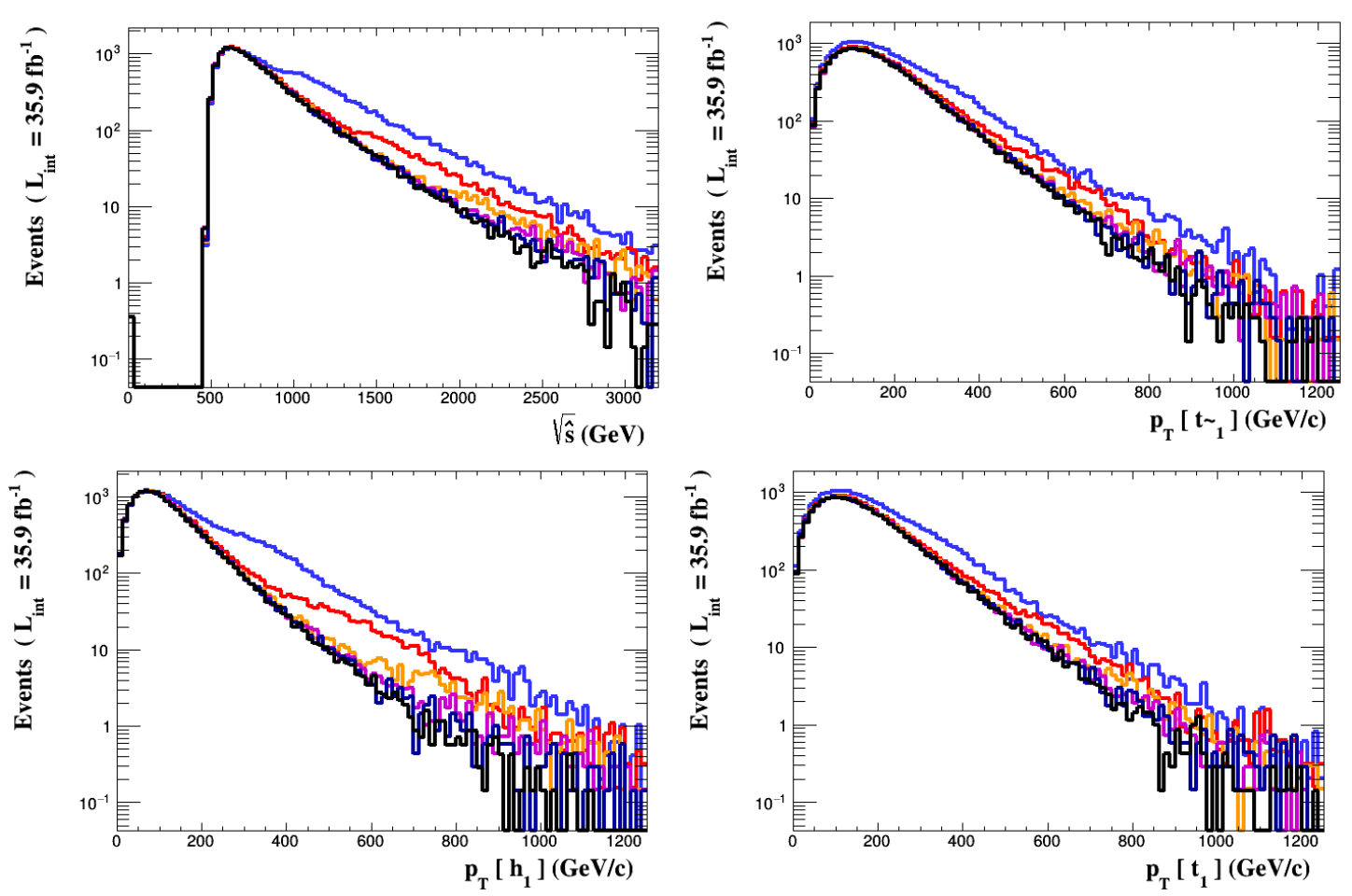

Figure 5.12: Distribution functions for the $t \bar{t} h$ process with MCHM5 form factors. Signal simulation done in Madgraph and kinematic analysis in Madanalysis. The black curve corresponds to the $\mathrm{SM}$ and the other ones are tabeled in table 5.2. Above to the left we have the $\sqrt{s}$ of the process. The one on the top right and the two of the bottom are $p_{T}$ distributions. At the bottom and to the right, we can see that the transverse momentum of the Higgs have significant deviations in shape from the SM, as is the case for $\sqrt{s}$.

Using MadAnalysis [100], we can obtain the distribution functions. In figures 5.12-5.13, we display the distributions for $\sqrt{s}$, transverse momentum, pseudorapidity $\eta$ and the Higgs-top and Higgs-top-top invariant masses.

From the cross-section results, one can see that the effect of dynamical interactions does not entirely decouple. There is an enhancement effect due to the pole structure of form factors, which is significant compared to $\mathcal{O}(\xi)$ corrections. The misalignment suppression competes with such effect leading to an overall enhanced cross-section for $f_{\mathrm{s}}$ below the $2-3 \mathrm{TeV}$ range. When the scale $f$ is large compared to the momentum flow, the results come back to non-dynamical composite Higgs suppression. 

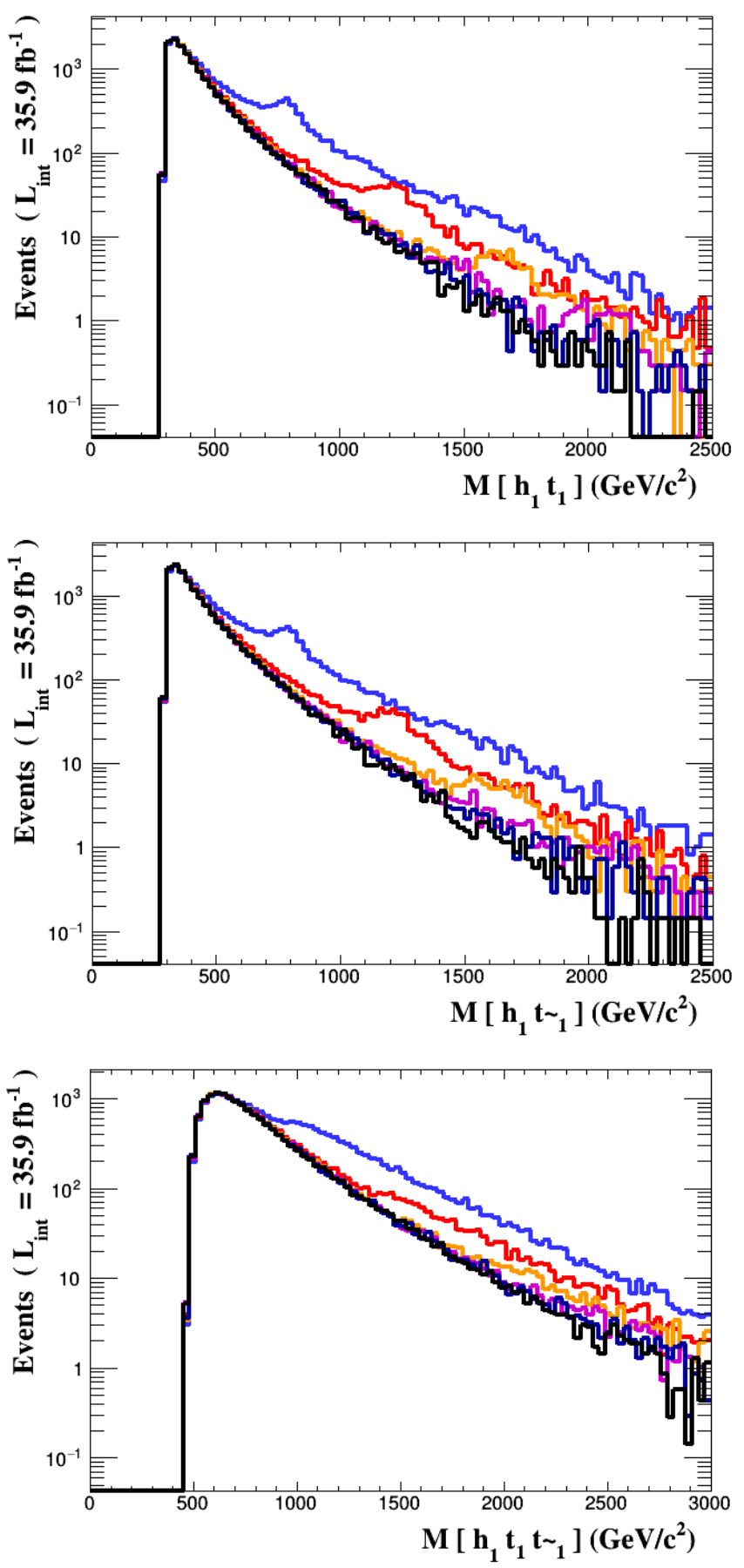

FiguRE 5.13: Distribution functions for the $t \bar{t} h$ process with MCHM5 form factors. Signal simulation done in Madgraph and kinematic analysis in Madanalysis. The plot is normalized to the SM prediction. The black curve corresponds to the SM and the other ones are shown in table 5.2 . The most sensitive probe to momentum dependence are invarian mass distributions. Here we can see the sharp peaks that are characteristic of the resonances. Even though these resonances are not explicitly put in the theory, through the non-linear form factors we can still see their effects at colliders. 


\begin{tabular}{|l|ccc|}
\hline Curve & $\mathbf{f}[\mathrm{TeV}]$ & $\mathbf{m}_{\mathbf{1}}[\mathrm{TeV}]$ & $\mathbf{m}_{\mathbf{4}}[\mathrm{Tev}]$ \\
\hline Blue & 0.8 & 0.9 & 1.2 \\
Red & 1.2 & 1.4 & 1.8 \\
Yellow & 1.6 & 1.9 & 2.5 \\
Pink & 2 & 2.4 & 3.1 \\
\hline
\end{tabular}

TABLE 5.2: Reference for the kinematic distribution figures 5.12-5.13. All widhts are $10 \%$ of the masses.

Thus, decoupling the dynamical interactions seems to be a hard task if the Higgs is assumed to be light.

Even though we are limited to partonic effects, without a complete study of the backgrounds, the kinematic modifications due to form factors are evident in our figures. It is through this formalism that we achieved the modifications of distribution functions expected in a composite scenario.

A very similar phenomenology should appear in the $Z h$ channel, in which a $Z$ boson goes off-shell like the intermediate top-quark here. Now, we shall proceed to see how form factors alter other channels of Higgs production. 


\subsubsection{The ggh Channel}

Even though it is a loop process, gluon fusion is the most efficient way of producing a Higgs. Because of this high production rate, ggh is the most explored channel of Higgs production so far in the LHC [9].

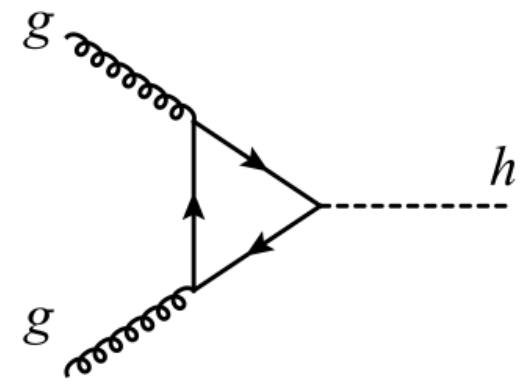

Such channel presents several opportunities to form factor models. First, the analysis of the Higgs off-shellness might provide the means to access a form factor that couples with the Higgs momentum. Furthermore, if we choose a form factor such as the Yukawa in the MCHM5, we alter the structure of the loop integral itself. This will lead to a zero momentum shift in the total production ratio of the whole channel.

However, because of the loop integral, complications arise when trying the complicated expressions of the MCHM5. Furthermore, without assuming the existence of a scalar mixing, the off-shell Higgs case does not provide significant alterations when including form factors. This remark is because the form factors derived in MCHM5 only see the top quark momentum and not the Higgs momentum.

For these reasons, we shall depart from the construction of form factors in the MCHM5 and follow the lines of the simpler ansatz expressions. These illustrate a central point of this work - a composite Higgs should no be limited to the prescription of Composite Higgs Models. Those are simply specific implementations of the compositeness idea itself. 


\section{Higgs Off-Shell}

Through gluon fusion we can explore the Higgs off-shelness [39]. Consider the contribution $g g \rightarrow Z Z$, in which the s-channel Higgs diagram is present together with the fermion box.

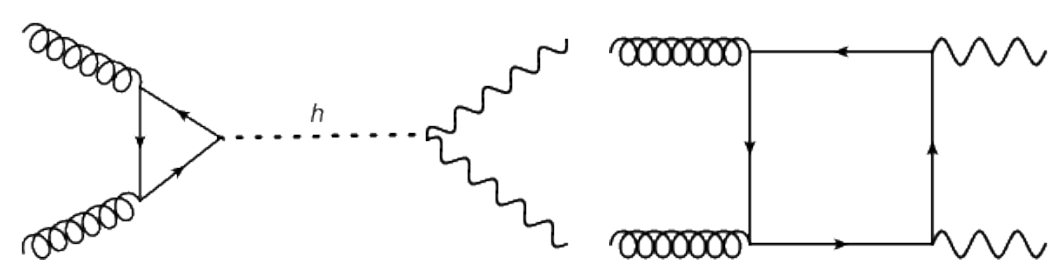

The desired signal comes from the Higgs diagram, but the box diagram comes together as an irreducible background. The two diagrams constructively interfere and provide exciting features for the study of form factors [38, 91].

A study of form factor effects in $g g \rightarrow Z Z$ was conducted by the authors of [38]. There, they used an ansatz for parametrization of the Higgs momentum dependence as a multipole - a structure analogue to the nucleon electromagnetic form factors.

$$
\Gamma\left(k^{2} / \Lambda^{2}\right)=\frac{1}{\left(1+k^{2} / \Lambda^{2}\right)^{n}}
$$

This structure presents differences to the MCHM example. First, the form factor sees the Higgs momentum, whereas in CHM it couples to the top quark momentum. There it was a direct consequence of PC, while here we are assuming another type of UV completion. Regardless of the completion, the form factor (5.6) should be taken only as an educated guess of an unknown realization of the Higgs compositeness.

Additionally, the form factor is in the kinematic region in which there are no poles. This euclidean behaviour is realized in hadronic studies and only provide suppression effects to the distribution functions. The order of the pole is governed by $n$, and the higher multipoles will produce slightly more visible effects as $n$ grows.

For the multipole form factor, the authors explored the opportunity of interference between the two diagrams. It turns out that the interference is constructive, 

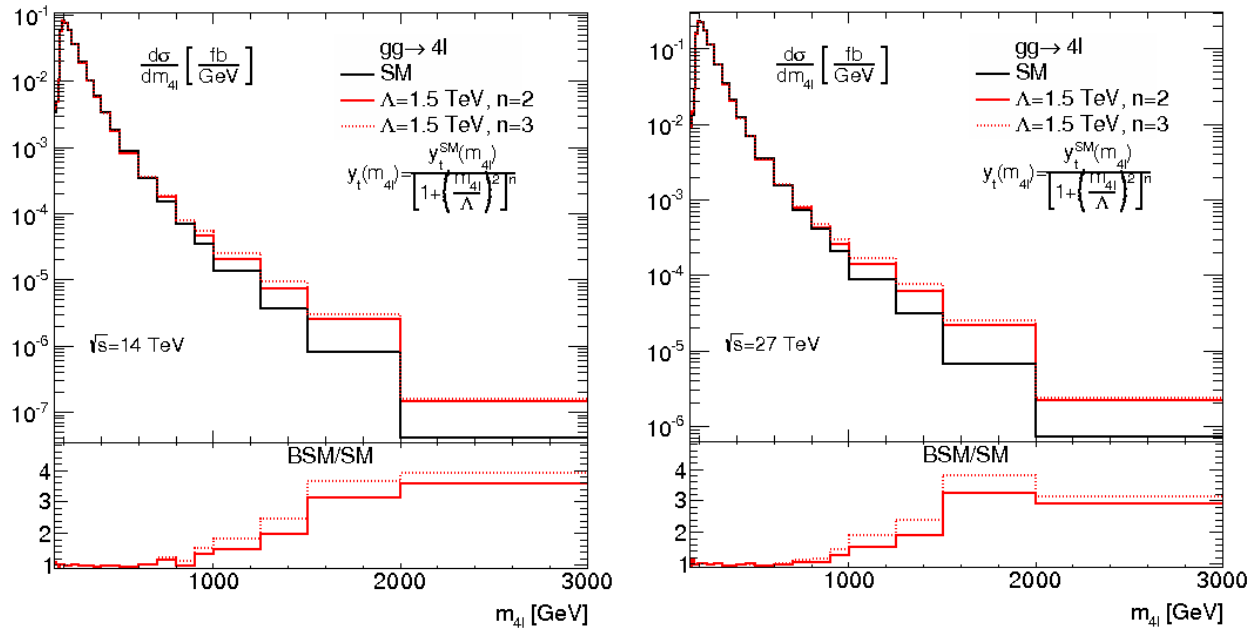

Figure 5.14: Analysis of a form factor model that couples with the Higgs momentum done by [38]. Four-lepton invariant mass distribution for the $g g \rightarrow 4 l$ process at the LHC $14 \mathrm{TeV}$ (left) and $27 \mathrm{TeV}$ (right). The SM prediction is the black curve. With the presence of a form-factor in the Higgs-top coupling, the distribution tail is modified for a strong sector scale of $\Lambda=1.5 \mathrm{TeV}$ (red).

making the Higgs and box diagrams enhance the signal. This effect made it possible to predict feasible signals in distribution functions for the $14 \mathrm{TeV}$ HL-LHC, and 27 TeV HE-LHC upgrades.

Using on-shell constraints, they limited the cutoff to $\Lambda>1.5 \mathrm{TeV}$. Since the signal strength of on-shell $Z Z^{(*)}$ final state is of order $\mathcal{O}(10 \%)$, they secured that $\left|\Gamma\left(m_{h}^{2} / \Lambda^{2}\right)^{2}-1\right|<0.1$ at $95 \%$ C.L. to satisfy on-shell constraints ${ }^{1}$.

As shown in figure 5.14, a form factor that modifies the interaction with the Higgs momentum is accessible through off-shell measurements. In such the form factor is accessed via the Higgs, and the constructive interference with the box diagram provides an effective method for enhancing the signal. As it is, these effects in distribution functions should be accessible in the next rounds of the LHC.

\footnotetext{
${ }^{1}$ The complete vertice here is $V_{t t h}=\frac{\sqrt{2} m_{t}}{v} \Gamma\left(k^{2} / \Lambda^{2}\right)$, normalized to match the SM prediction as $\Lambda \rightarrow \infty$.
} 


\section{Modification of the Loop Integral}

Shifting from a form factor that couples with the Higgs momentum to one that couples to the top-quark momentum, we are reproducing a structure more similar to the MCHM5. Here, when the top quark momenta are modified, the gluon fusion process integral is also modified even for the on-shell Higgs.

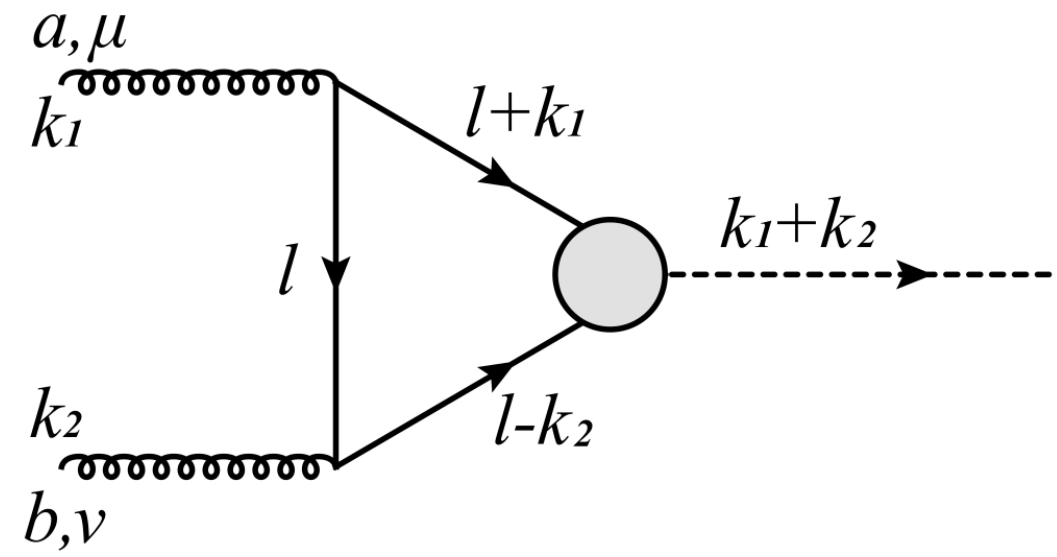

FiguRE 5.15: Gluon loop for Higgs production with the form factor. One can see why the form factor must couple to the top-quarks momenta - If it couples to the Higgs, the integration on loop momenta is the same, leading to just a normalization factor.

First, we define a simple ansatz for the form factor here.

$$
\begin{aligned}
& \Gamma\left(p^{2}, q^{2}\right)=F\left(p^{2}\right)+F\left(q^{2}\right) \\
& F\left(p^{2}\right)=\left(\frac{-i y_{t}}{\sqrt{2}}\right) g_{\xi} \frac{-M^{2}}{p^{2}-M^{2}}
\end{aligned}
$$

where $y_{t}$ is the top-Yukawa coupling and $g_{\xi}$ is a misalignment coefficient.

Figure 5.15 describes the loop. The amplitude for this process is

$$
\mathcal{M}=2\left(-i g_{s}\right)^{2}\left(\frac{-i y_{t}}{\sqrt{2}}\right) i^{3} \operatorname{Tr}\left[t^{a} t^{b}\right] \epsilon_{\mu} \epsilon_{\nu} I^{\mu \nu}
$$

where $I^{\mu \nu}$ is

$$
I^{\mu \nu}=\int \frac{d^{4} l}{(2 \pi)^{4}} \frac{F\left(l+k_{1}\right)}{D_{1} D_{2} D_{3}} \operatorname{Tr}\left[\left(l_{k / 1}+m\right) \gamma^{\mu}(l+m) \gamma^{\nu}\left(\not l-\not l_{2}+m\right)\right]
$$


One can define clearly the path to complete this calculation. First, it is necessary to reduce the trace. Then, to put the integral into a known format, use Feynman parametrization and dimensional regularization. The last step is to use the Passarino-Veltman (PV) reduction reduce the types of tensor integrals we get to scalar ones.

Fortunately, we can use the FORMCalc program [101] to perform the (PV) reduction. We show in figure 5.16 the results after the calculation of the averaged amplitude to the square.

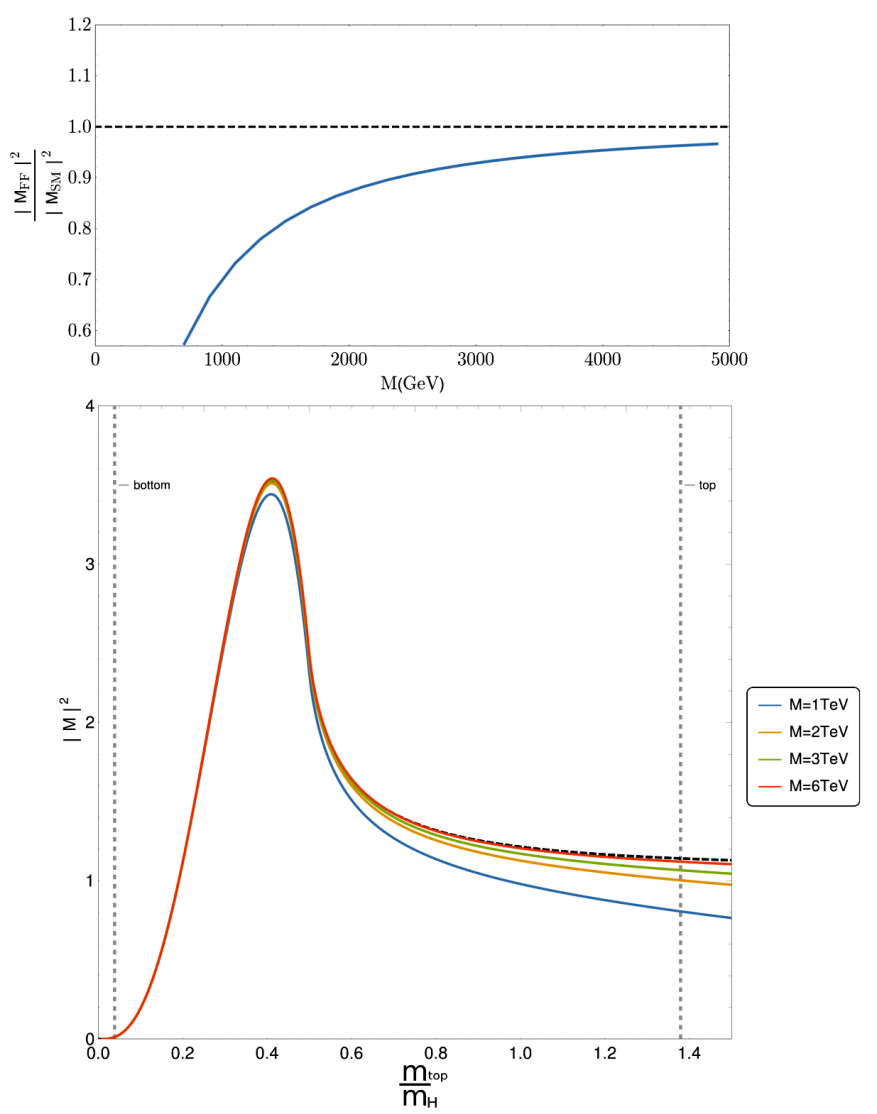

FigURE 5.16: Numerical evaluation of the gluon fusion integral in the form factor case. Due to the euclidean momentum structure of the loop, there is no enhancement since we are far away from the pole region. The upper plot shows how the cross section would scale with the mass of the heavy top partner, and the bottom one shows the integral averaged and squared as a function of $m_{t} o p / m_{h}$. Compared to the SM calculation of [78], the integral seems to go correctly to the SM limit as $M$ grows.

From the analytical point of view, the momentum circulating in the loop integral is at the euclidean kinematic regime. When performing dimensional regularization, the necessity of wick rotating indicates such behaviour. Physically, this 
kinematic region of the form factor means that in the loop, we are not accessing the physical poles of the resonances. So, after integrating, there is a suppression effect.

Even though this form factor is not realistic, it illustrates what would happen in the MCHM5 case. The kinematic region which the form factor access in the loop is not close to the physical threshold and produces further suppression.

Because of the difficulty in performing these calculations numerically, we could not study the MCHM5 complex form factor structure. The most interesting case, studying the off-shell Higgs with modifications to the loop integral is a difficult task. We shall postpone this study to future research.

\subsubsection{Other Channels}

The most promising channel that we left out of our analysis is $Z H$. In it, a $\mathrm{Z}$ boson "strahlungs" to produce another $\mathrm{Z}$ and a Higgs. This is the same structure as in tth, and we expect to obtain similar results in such study. ${ }^{2}$

Additionally, the other channels offer some difficulties. Even though vector boson fusion has a large cross-section compared to other channels, its reach is determined by detector capabilities. In weak boson fusion, the central collision is highly central and depends on more intricate jet detection techniques [93]. However, the off-shell Higgs in this channel presents exciting possibilities.

\footnotetext{
${ }^{2}$ At the current moment, we are studying the form factors effects on the $Z H$ channel, but their inclusion in the dissertation was not possible.
} 


\subsection{Compositeness from the Collider Perspective}

For CHMs, the inclusion of form factors as dynamical effects in interactions considerably enrich the phenomenology. The parametrization with momentum dependence not only is necessary but is indispensable for compositeness scales at the few $\mathrm{TeV}$ ranges.

The in-depth analysis of collider simulation and hadronization should be achieved in our future research. However, the vital core of the phenomenological predictions is already present here - form factors modify the distribution functions according to a well defined physical prescription. Analyticity of external momentum was a guide in establishing the anatomy of form factors, and the prescription we presented is a concrete realization of the Higgs compositeness.

Since this realization is a particular, QCD-biased implementation, one can ask for a more general picture of the Higgs compositeness. This exploration shall be the last topic in our discussion of the composite Higgs and should also serve as a compass for future research. 


\section{Chapter 6}

\section{Discussions and Conclusion}

Previously, we discovered that the necessity of momentum dependence in composite Higgses extended the CHM scope of signals. As defined, the zero momentum parameterization of a CHM hides away attributes like the momentum dependence of three-point interactions. Although concrete, the effective models without momentum dependence are, perhaps, too simplistic. Their local structure does not fully capture the dynamical content of the strong sector.

In light of what we discovered, we can conclude that the restoration of dynamical effects in the non-local form factor approach catches the main features of the Higgs compositeness. Consequently, the composite Higgs picture can be upgraded according to the theoretical and phenomenological expectations of such a scenario.

Our approach had the main limitation of being very QCD-based. An interesting exercise is to speculate about how a strong sector for the Higgs could be different from QCD. This will lead us to the prospects in future research of such formalism presented throughout the dissertation. The idea is to build-up the phenomenology of the different ways of compositeness, while exploring in more general terms the analytic structure of form factors. 


\subsection{The Composite Higgs Picture}

To construct a picture of a composite Higgs, we needed mainly three elements. First, the formalism for dealing with the symmetry structure of a the pNGB Higgs and all the CHM effective parametrization. Then, it was necessary to introduce further hypothesis on the interactions of the elementary and composite sectors via partial compositeness and gauged external sources. Finally, the dynamical interactions were constructed to match the specifications of the spectral decomposition and analytic structure of the theory.

As a rule, each part introduced a general feature of compositeness but also approximations and assumptions. To point to the general picture, we must trace back some steps, and find the generalities and the expenses of the model-building choices we made.

When constructing a composite Higgs, there should be a scale that dynamically generates a composite sector. To build an effective theory, we need to respect, at least approximately, some global symmetry structure.

If the Higgs is composite, an essential feature is that it needs to be light compared to the compositeness scale. This necessity comes from experimental observation - there are not any new states lying around the Higgs scale. Here, making the Higgs a pNGB is a straightforward way of achieving such lightness.

While the existence of a global symmetry is general and the pNGB Higgs is desired, the specific group realization is particular to each model. Choosing such symmetry breaking pattern as $S O(5) \rightarrow S O(4)$, or specific fermionic representations is a model-building requirement. Overall, these specific choices lead to similar phenomenology - a misalignment suppression that is a function of $\xi=v^{2} / f^{2}$.

This fixes the picture of the effective theory that shares the SM degrees of freedom. Symmetry structure is an essential guide, the pNGB is a desired tool, and the specific group assignments are just specific realizations of the idea. 
Then, the inclusion of dynamical effects comes from assuming the composite/elementary interactions. This part is responsible for obtaining an adequate dynamical EWSB, with a naturally light Higgs. Thus, the importance of such interactions lies in the resolution of the hierarchy problem.

In general, the connection of elementary and composite sectors is made with operators that are generated by some UV completed theory. As we argued in chapter 2, the scaling dimensions of this operator must be such that there is not a reintroduction of the HP later on.

The study of these operators has led us to partial compositeness, a commonly used framework in $\mathrm{CH}$ phenomenology. With PC, elementary and composite states mix and provide means to build our form factors later on. However, this entire theoretical construction should not be viewed as something more generic than a hypothesis - There could be other constructions that relate the two sectors and dynamically generate EWSB. Admittedly, they are far less common in literature ${ }^{1}$, and novel ideas are truly needed.

Finally, in trying to include dynamical effects in the Higgs phenomenology, we stumbled into the spectral decomposition. Such prescription is the most general expression for a two-point interaction in any theory, regardless of interaction strength. However, to extract functional forms from the spectral decomposition, we had to impose plenty of approximations.

First of all, the use of sum rules can be justified as the necessity of $\mathcal{G}$-symmetry restoration in the UV. This use can be viewed as a general feature of a composite Higgs since we are taking for granted the existence and reliability of such symmetries.

Furthermore, the narrowness of resonances was crucial to factor their contribution from the complicated continuum of the spectral function. We justified this assumption through the QCD-biased $1 / N$ expansion, yet much space for alternatives remains. Even in QCD, the large-N behaviour is not capable of explaining some details like the wide $\sigma$ 's and $\rho$ 's, and their strongly interacting behaviour at the

\footnotetext{
${ }^{1}$ Technicolor being the most notable alternative, despite its failures in making a viable BSM candidate.
} 
hadron level. Conversely, the large-N should be viewed at best as an organizational principle.

Additionally, throwing away the towers of resonances and the continuum seems to be just a simplification, without a deep justification. There, the focus was on obtaining an explicit formulation and achieving concrete results. Nevertheless, it provided much insight into the phenomenological capabilities of our approach.

Most strikingly, as we try to go past the 't Hooft limit, we face the enormous puzzle of the scope of strongly interacting theories. Beyond the formation of bound states, we have limited knowledge of the capabilities of the nonperturbative physics. In such a regime, the narrow and free behaviour of composite particles can be questioned, and even resonant phenomena are not granted.

Related to the problem of nonperturbative methods, it remains the inclusion of a language for three-point functions. Something like a spectral decomposition, which relates physical properties to analyticity in external momentum is not available for three-point functions. In the case of these interactions, we expect a more complex structure to arise, reflecting the kinematical possibilities and all intricate vertex contributions from multiparticle states.

While there is not a general decomposition of three-point functions, we build the form factors from the assumption of elementary and composite sectors interactions. Then, because the Higgs becomes non-dynamical and generates two-point contributions as masses for all elementary particles, we could adequately normalize the form factors and impose phenomenological constraints.

We can see a scheme for the picture in table 6.1. In short, a composite Higgs is described by a $\mathcal{G}$-effective theory, that dynamically generates EWSB through explicit breakings and respects physical constraints on its interactions. Beyond that, we are subject to the further hypothesis that forms different models of composite Higgses. 


\begin{tabular}{|ccc|}
\hline General & Hyphothesis & $\begin{array}{c}\text { Simplification } \\
\text { Model Building }\end{array}$ \\
\hline $\begin{array}{c}\text { Exact/approximate } \\
\text { global } \mathcal{G}\end{array}$ & pNGB Higgs & $\begin{array}{c}\text { Specific realizations } \\
\text { i.e. } \mathcal{G}=S O(5), \psi=\mathbf{5}^{\prime} s, \ldots\end{array}$ \\
\hline $\begin{array}{c}\text { Dynamical EWSB, } \\
\text { light Higgs }\end{array}$ & PC and mixtures & Resonance content \\
\hline $\begin{array}{c}\text { Unitarity, analiticity, } \\
\text { spectral decomposition, } \\
\text { sum rules. }\end{array}$ & $\begin{array}{c}\text { Formation of resonances, } \\
\text { confinement, } \\
\text { large-N limit. }\end{array}$ & $\begin{array}{c}\text { Only first resonance, } \\
\text { discarting the continum. }\end{array}$ \\
\hline
\end{tabular}

TABLE 6.1: The composite Higgs picture. Scheme of all general features, hyphotesis and simplifications/model-building choices we made throughout the text.

\subsection{Perspectives}

Throughout the $\mathrm{CH}$ literature, there have been many alternative realizations, each one with different mechanisms for the generation of a light Higgs [29]. These were intensely explored before, and are not the main focus of our work. Alternatively, we should focus on the form factor possibilities, beyond the approximations discussed in the last section.

The exploration of momentum dependence in more exotic scenarios could provide examples of different ways a Higgs can behave as a composite particle. In general, such scenarios are created by strongly interacting sectors that depart from QCD-based behaviour.

Here, we shall list some possibilities for these sectors. The tone of this discussion is exploratory, intending to present prospects for future applications of form factor phenomenology. Even though the consequences are not carried out thoroughly, these possibilities will help to build a complete picture of the composite Higgs possibilities.

\section{Invisible Resonances}

It is a possibility that the heavy resonances are simply not seen by colliders. There are some ways in which this feature could be true. 
Suppose a scenario in which the strong sector has a hidden part that is neutral to the SM groups. Then, the spectrum of the theory could include particles that are invisible to our ordinary matter detectors. Evidently, in such case, the mass scale of the hidden partners of SM particles could be much lower since the effects of resonances go to invisible collider contributions.

A concrete realization of such a scenario is the Twin Higgs model [40]. Remarkably, the Twin Higgs also addresses the hierarchy problem through the idea of neutral naturalness [41]. In it, the invisible top-quark partners are responsible for cancelling the quadratic divergences of the SM, a cancellation that is a consequence of the symmetry structure of the theory.

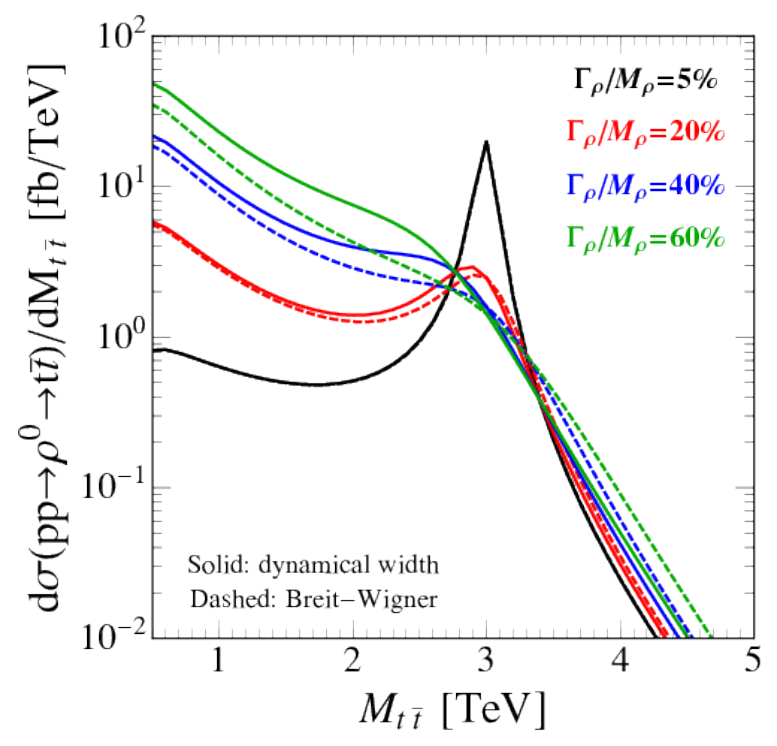

FiguRE 6.1: Shape of a broad composite resonance at the LHC. Assuming the s-channel production of a $3 \mathrm{TeV} \rho$ ressonance, [43] studied the effects of increasing widths in kinematic distributions. Note that for increasing widths, the characteristic sharp peak distributions are broaden to becoming enhanced rates.
Another possibility for making heavy partners invisible to experiment is by avoiding the resonant behaviour. If the resonances start getting very broad compared to their masses, their clear experimental signatures through sharp peaks start getting obscured. A broad resonance would only be seen as an enhanced rate, which is an experimentally challenging probe.

In the MCHM5, the resonances narrowness is justified since they decay only to SM states, and the strong sector by itself is assumed to be stable in the large-N limit. For example, the coupling of the $\mathrm{TeV} \rho$ with the $\mathrm{SM}$ is suppressed by $\rho$-SM mixing, which guarantees its 
stability. However, in allowing for other embeddings, the narrow character of resonances could come up as a generic feature. This possibility is discussed in [43].

As resonance widths become larger compared to their masses, deviations of the Breit-Wigner shape become more apparent. To treat broad resonances beyond the leading narrow width approximation, one could replace the propagator as

$$
\frac{1}{\left(s-M_{\rho}^{2}\right)^{2}+M_{\rho}^{2} \Gamma_{\rho}^{2}} \rightarrow \frac{1}{\left(s-M_{\rho}^{2}\right)^{2}+s^{2} \Gamma_{\rho}^{2} / M_{\rho}^{2}}
$$

In the direct s-channel production of the $\rho$, replacing the propagator produced the invariant mass distributions in figure 6.1. As expected, the sharp peak signal in distributions become more elusive to detection.

A possibility then is to assume broad resonances in the form factor expressions. In a realization of the $\mathrm{CH}$ that allows large widths, making the substitution (6.1) into the expressions of the derived form factors should produce more subtle signals in the presented channels here. The pivotal question is to what extent they are still detectable. Such possibility shall be considered for future developments.

\section{Continuum Contributions and Unparticles}

At some point, as the resonances keep getting broader, their contribution cannot be integrated out from the spectral function and become part of the continuum distribution. In the analytic plane, their poles are getting shifted out from the physical region of real masses. Such a situation implies that, at some point, resonances cannot be described as particles anymore since their masses are not well defined.

As an example, despite its role in chiral perturbation theory and nucleon-nucleon interactions, much controversy arises as of the existence of the $\sigma$ meson in QCD. The pole position of the $\sigma$ is measured to be at $\sqrt{449_{-16}^{+22}-i(275 \pm 12)} \mathrm{MeV}$ giving a width of $\sim 60 \%$ the value of the mass [65]. Hence, when looking at experiments, the sigma cannot be detected as an isolated Breit-Wigner distribution, but only through phase shifts measurements, as indicated in figure 6.2. 


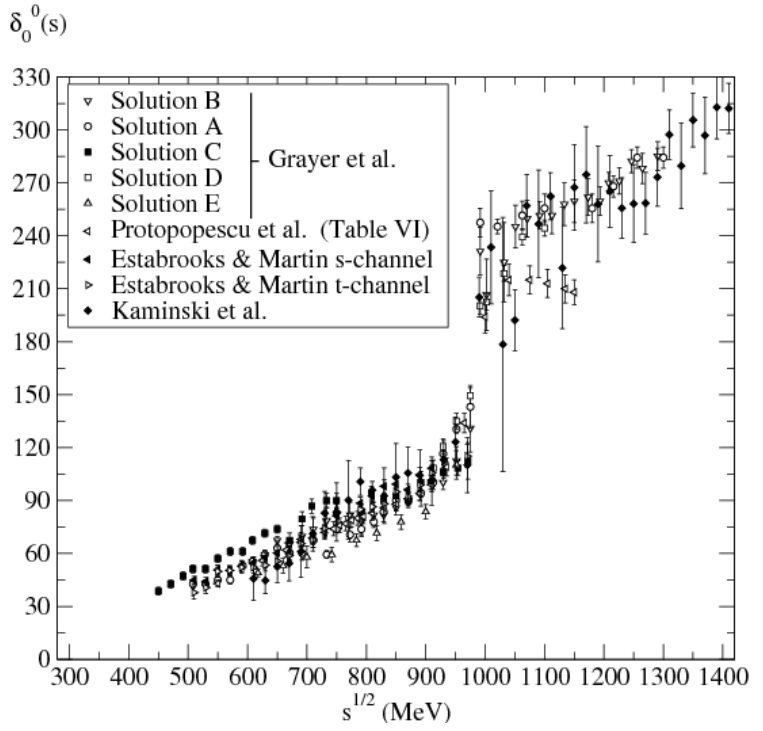

Figure 6.2: Phase shift of $\pi \pi \rightarrow \pi \pi$ process. The sigma is so wide that the familiar BreitWigner is not appropriate to describe its behaviour. There is no phase shift at $500 \mathrm{MeV}$, the mass of the sigma. This is in contrast with the sharp phase increase of the $f_{0}(980)$ resonance.
As a consequence of the unfitting status of the $\sigma$, it is assigned as a nonordinary quark-antiquark meson. Its description indicates the need to go beyond the $1 / N$ expansion, into more exotic scenarios. The large- $\mathrm{N}$ is insufficient even within QCD, where it fails to provide some important aspects of the hadronic theory.

Assuming such an elusive strong sector hinders the detection possibilities. However, a concrete implementation of such ideas for a composite Higgs could attack the hierarchy problem. Because broad resonances are harder to see, the experimental bounds get loosened, and these states can be generically lighter. The consequence is less fine-tuning and a more natural model-building.

Previously, the description of the continuum spectral function was attributed to multiparticle contributions. While multiparticle states surely contribute to a continuous momentum dependence, the whole continuum part of the spectral function can have more internal dynamics. An exotic scenario, described by Howard Georgi, provides an example of a theory in which there are no particles states.

The idea of unparticle correlates strongly with scale invariance. Georgi idea relates to "stuff" that is scale-invariant while not necessarily having zero mass. These are generally members of the continuum spectrum of the theory. 
Then, the unparticle sector is coupled to the SM at low energies via interaction terms like ${ }^{2}$ :

$$
\mathcal{L}_{\text {int }}=c_{1} \bar{e} \gamma_{\mu} e O_{\mathcal{U}}^{\mu}+c_{2} \bar{e} \gamma_{\mu} \gamma_{5} e O_{\mathcal{U}}^{\mu}
$$

where $O_{\mathcal{O}}^{\mu}$ is the unparticle operator.

Even though we do not intent to present the details, the unparticle spectral function is easily obtained by scale invariance requirements. It is constrained to scale with dimension $2 d_{\mathcal{U}}$, the dimension of $O_{\mathcal{U}}^{\mu}$.

$$
\left|\left\langle 0\left|O_{\mathcal{U}}(0)\right| p\right\rangle\right|^{2} \rho\left(p^{2}\right)=A_{d_{\mathcal{U}}} \theta\left(p^{0}\right) \theta\left(p^{2}\right)\left(p^{2}\right)^{d_{\mathcal{U}}-2}
$$

Which lead to an integrable example of a continuous spectral function. ${ }^{3}$

$$
\begin{aligned}
\Pi_{\mathcal{U}}\left(p^{2}\right) & =\int_{0}^{\infty} d m^{2} \frac{\rho\left(m^{2}\right)}{p^{2}-m^{2}} \\
& =i \frac{A_{d_{\mathcal{U}}}}{2} \frac{g^{\mu \nu}+p^{\mu} p^{\nu} / p^{2}}{\sin \left(d_{\mathcal{U}} \pi\right)}\left(-p^{2}-i \epsilon\right)^{d_{\mathcal{U}}-2}
\end{aligned}
$$

The important point about (6.5) is that it provides an explicit continuum effect that could be used in the form factor formalism. Ultimately, the main use of these exotic possibilities is to see how much sensibility to UV physics we can have in phenomenology when its effects are highly elusive. However, as theoretical subjects, scale-invariant sectors do propose an interesting framework for the Higgs compositeness.

Remarkably, conformal invariance is found in many applications of CHMs. An important result regards the 4d EFT we studied here is equivalent to a holographic 5 description in the so-called Randall-Sundrum models [66, 67, 68, 69]. In most developments of holographic models, the strongly interacting sector of the Higgs is assumed to have conformal symmetry.

\footnotetext{
${ }^{2}$ Georgi proposes this tensor structure as just an example of unparticles applications.

${ }^{3}$ In which $A_{d_{\mathcal{U}}}=\frac{16 \pi^{5 / 2}}{(2 \pi)_{\mathcal{U}}^{d}} \frac{\Gamma\left(d_{\mathcal{U}}+1 / 2\right)}{\Gamma\left(d_{\mathcal{U}}-1\right) \Gamma\left(2 d_{\mathcal{U}}\right)}$. For more details check [80].
} 
It seems that the possibilities we presented before can fit in the context of a conformal strongly interacting sector. This scenario presents a theory which is asymptotically free in the UV, but a non-trivial fixed point dominates the infrared, and model-building is explored through the use of dualities like the AdS/CFT correspondence [70]. Generically, broad resonances and continuum contributions can be attainable near conformal windows, where the theory parameters run very slowly from the IR to the UV.

These exotic possibilities suggest that the Higgs could be composite in different ways beyond the usual large-N prescription. However, since we do not dispose of a lot of nonperturbative tools, exploring strongly interacting theories has proven to be an enormous challenge. Ultimately, having a grasp of the landscape of possible completions is an impossible task.

However, there might be a better strategy than guessing specific behaviours of these composite sectors. Here, an interesting idea follows along the lines of the Bootstrap philosophy.

Most of the developments achieved in the momentum dependent implementation rely on imposing constraints on the form factors. We used the specifications of the spectral decomposition to limit the possibilities of two-point functions and to provide certain realizations for three-point form factors.

Imposing boundary conditions on form factors seems to be an effective way of taming the complicated three-point functions kinematic dependence. Something reminiscent of the Heisenberg's Bootstrap program could be of use here - Using unitarity, analyticity, causality and the spectral decomposition to constraint form factor behaviour.

While trying to make sense of nonperturbative physics, the Bootstrap models became popular models in the '60s and '70s. At that time, the idea was to solve or constraint the amplitudes using only basic principles of field theory, without any perturbative means. While the Bootstrapping became a tool of QFT framework, the program was mostly abandoned after the experimental discovery of quarks and 
gluons.

In modern developments, Heisenberg's idea would return through the Conformal Bootstrap [81, 82]. The same premise is applied here, to study strongly coupled theories nonperturbatively using symmetries and other consistency conditions. In the case of conformal field theories, the conformal Bootstrap was able to achieve some interesting results.

In two-dimensions, Poincaré invariance and the conformal transformations were sufficient to solve various $2 \mathrm{~d}$ models. With only the general axiomatic assumptions of the Bootstrap program, the two- and three-point functions were significantly constrained.

More recently, in 2008, the conformal Bootstrap was developed for $4 \mathrm{~d}$ theories [83]. Historically, one of the primary motivations for using bootstrap techniques in $4 \mathrm{~d}$ was to shed light into the Hierarchy Problem. The goal was to alleviate the dangerous quadratic behaviour of the Higgs mass term by increasing its scale dimension. As we have seen, a similar strategy was used in the Composite Higgs and Technicolor models.

The Bootstrap philosophy gives an enormous playground for model-building. Using the form factor approach, the requirement of physical conditions like unitarity and analyticity seems to be a compelling approach to limit their behaviour. The conformal Bootstrap presents a remarkable example of how theoretical investigations can constraint strongly interacting scenarios for BSM physics. 


\subsection{Conclusion}

In conclusion, through the use of form factors, we could extract collider signals of the Higgs compositeness. To achieve our goal, we had to restore the full momentum dependence of the three-point interactions of the Higgs in the form factor formalism.

Particularly, we used the MCHM5 as an object of study. Through an inquiring review of CHM, we could identify the lacking element of the Higgs compositeness. Within the form factor formalism, the point-like interactions of the CHM got promoted to dynamical interactions, with a momentum dependence associated with them. Such momentum dependence was explored according to the general nonperturbative prescriptions available, like the spectral decomposition.

Within the MCHM5, the numerical implementation showed that form factor effects add to the phenomenology of the zero momentum parametrization. On CHMs, the only coupling modifications are due to misalignment suppression. Here, we found that the pole structure of form factors enhances this contribution at the $\mathcal{O}\left(p^{2} / m_{*}^{2} \sim \xi\right)$ order. Due to this effect, the dynamical enhancement in the crosssection is at the same order of the misalignment suppression, as indicated in figure 5.11. Momentum dependence not only enriches the phenomenology but is indispensable to usual $\mathrm{CH}$ models.

Importantly, the form factor study was capable of modifying the shape of the distribution functions - an expected feature of compositeness. These modifications presented us with exciting phenomenological signals, with important prospects for more detailed studies.

The developments made in our project presented many opportunities for future research.

Theoretically, the possibilities for the Higgs compositeness are many. Previously in this chapter, we have dedicated a discussion of such possibilities, ranging from neutral composite sectors to broad resonances and unparticles. The conformal sector exploration is a vast and rich subject, which leads to interesting phenomenology and 
a robust framework for model-building. Bootstrapping constraints on form factor expressions is an exciting idea that we wish to implement in our discussions.

The HP stands as the ultimate difficulty of the SM framework. However, the tone we adopted is to shift from hierarchy to the Higgs compositeness. This methodology can, perhaps, help elucidate the problem with novel strategies to attack it. Alternatives for the Higgs compositeness might lead to novel ways of viewing the HP, with new methods and tools.

Phenomenologically, we have identified off-shellness as the necessary condition for the appearance of momentum dependent effects. The experimental analysis of such processes is intricated but full of possibilities. By having off-shell momenta, we are probing the collider processes in their most UV sensitive form. If there BSM physics, off-shellnesss magnifies the potential of their discovery significantly.

The next step is to study these signatures even further, into the detailed simulation of the listed channels. To concretely find the reach of the LHC, we need to be able to perform a complete analysis of background and hadronization effects, together with the detector capabilities. The critical questions are - Is the LHC reach at HL and HE enough to find any Higgs compositeness? Furthermore, what are the bounds we can extract from these data? What about future colliders?

These questions are guiding principles to the next stages of our project. With or without BSM physics, the next phases of the LHC are already a concrete reality. At this important phase of particle physics, the community will shift its attention to these developments. Our major concern stands as being able to effectively extract the LHC signals, and this shall be our guide to future research. 


\section{References}

[1] Peter W. Higgs. "Broken Symmetries and the Masses of Gauge Bosons". In: Phys. Rev. Lett. 13 (1964). [,160(1964)], pp. 508-509. DOI: 10 .1103/ PhysRevLett.13.508.

[2] Steven Weinberg. "A Model of Leptons". In: Phys. Rev. Lett. 19 (1967), pp. 1264-1266. DOI: 10.1103/PhysRevLett.19.1264.

[3] Abdus Salam. "Weak and Electromagnetic Interactions". In: Conf. Proc. C680519 (1968), pp. 367-377. DOI: 10.1142/9789812795915_0034.

[4] S. L. Glashow. "Partial Symmetries of Weak Interactions". In: Nucl. Phys. 22 (1961), pp. 579-588. DOI: 10.1016/0029-5582(61)90469-2.

[5] J. Goldstone. "Field Theories with Superconductor Solutions". In: Nuovo Cim. 19 (1961), pp. 154-164. DOI: 10.1007/BF02812722.

[6] Riccardo Barbieri et al. "Electroweak symmetry breaking after LEP-1 and LEP-2". In: Nucl. Phys. B703 (2004), pp. 127-146. DOI: 10 .1016/j . nuclphysb . 2004.10.014. arXiv: hep-ph/0405040 [hep-ph].

[7] Georges Aad et al. "Observation of a new particle in the search for the Standard Model Higgs boson with the ATLAS detector at the LHC'. In: Phys. Lett. B716 (2012), pp. 1-29. DOI: 10 . 1016/ j . physletb . 2012 . 08 . 020. arXiv: 1207.7214 [hep-ex].

[8] Serguei Chatrchyan et al. "Observation of a New Boson at a Mass of 125 GeV with the CMS Experiment at the LHC". In: Phys. Lett. B716 (2012), pp. 30-61. DOI: 10 . 1016/j . physletb. 2012 . 08 . 021. arXiv: 1207 . 7235 [hep-ex]. 
[9] Albert M Sirunyan et al. "Combined measurements of Higgs boson couplings in proton-proton collisions at $\sqrt{s}=13 \mathrm{TeV}$ ". In: Eur. Phys. J. C79.5 (2019), p. 421. DOI: 10 . $1140 /$ epjc/s10052-019-6909-y. arXiv: 1809.10733 [hep-ex].

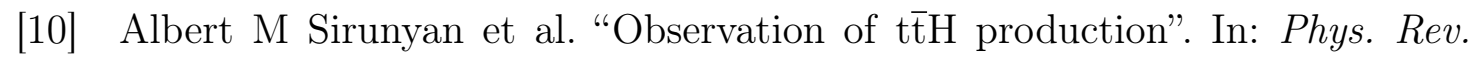
Lett. 120.23 (2018), p. 231801. DOI: 10 . 1103/PhysRevLett. 120 . 231801. arXiv: 1804.02610 [hep-ex].

[11] Albert M Sirunyan et al. "Running of the top quark mass from proton-proton collisions at $\sqrt{s}=13$ TeV". In: Phys. Lett. B803 (2020), p. 135263. DOI: 10.1016/j.physletb. 2020.135263. arXiv: 1909.09193 [hep-ex] .

[12] Michael E. Peskin and Tatsu Takeuchi. "Estimation of oblique electroweak corrections". In: Phys. Rev. D46 (1992), pp. 381-409. DOI: 10.1103/PhysRevD . 46.381 .

[13] Riccardo Barbieri. "Electroweak theory after the first Large Hadron Collider phase". In: Phys. Scripta T158 (2013), p. 014006. DOI: 10 . 1088 / 0031 8949/2013/T158/014006. arXiv: 1309.3473 [hep-ph].

[14] Peter W. Higgs. "Nobel Lecture: Evading the Goldstone theorem". In: Rev. Mod. Phys. 86.3 (2014), p. 851. DOI: 10.1103/RevModPhys.86.851.

[15] François Englert. "Nobel Lecture: The BEH mechanism and its scalar boson". In: Rev. Mod. Phys. 86.3 (2014), p. 843. DOI: 10.1103/RevModPhys. 86.843.

[16] Riccardo Barbieri. Ten Lectures on the Electro Weak Interactions. Scuola Normale Superiore, 2007. ISBN: 9788876423116. arXiv: 0706.0684 [hep-ph].

[17] Steven Weinberg. "Elementary particle theory of composite particles". In: Phys. Rev. 130 (1963), pp. 776-783. DOI: 10.1103/PhysRev.130.776.

[18] P. T. Matthews and Abdus Salam. "Relativistic field theory of unstable particles". In: Phys. Rev. 112 (1958), pp. 283-287. DOI: 10.1103/PhysRev.112. 283. 
[19] P. T. Matthews and Abdus Salam. "Relativistic theory of unstable particles. 2". In: Phys. Rev. 115 (1959), pp. 1079-1084. DOI: 10.1103/PhysRev. 115. 1079.

[20] David B. Kaplan and Howard Georgi. "SU(2) x U(1) Breaking by Vacuum Misalignment". In: Phys. Lett. 136B (1984), pp. 183-186. DOI: 10 . 1016 / 0370-2693(84)91177-8.

[21] David B. Kaplan, Howard Georgi, and Savas Dimopoulos. "Composite Higgs Scalars". In: Phys. Lett. 136B (1984), pp. 187-190. DOI: 10 .1016/0370$2693(84) 91178-\mathrm{X}$.

[22] David B. Kaplan. "Flavor at SSC energies: A New mechanism for dynamically generated fermion masses". In: Nucl. Phys. B365 (1991), pp. 259-278. DOI: $10.1016 / \mathrm{S} 0550-3213(05) 80021-5$.

[23] Kaustubh Agashe, Roberto Contino, and Alex Pomarol. "The Minimal composite Higgs model". In: Nucl. Phys. B719 (2005), pp. 165-187. DOI: 10 . 1016/j.nuclphysb.2005.04.035. arXiv: hep-ph/0412089 [hep-ph] .

[24] Roberto Contino, Leandro Da Rold, and Alex Pomarol. "Light custodians in natural composite Higgs models". In: Phys. Rev. D75 (2007), p. 055014. DOI: 10.1103/PhysRevD.75.055014. arXiv: hep-ph/0612048 [hep-ph].

[25] Alex Pomarol and Francesco Riva. "The Composite Higgs and Light Resonance Connection". In: JHEP 08 (2012), p. 135. DOI: 10.1007/JHEP08(2012) 135. arXiv: 1205.6434 [hep-ph].

[26] Roberto Contino, Yasunori Nomura, and Alex Pomarol. "Higgs as a holographic pseudoGoldstone boson". In: Nucl. Phys. B671 (2003), pp. 148-174. DOI: $10.1016 / j$. nuclphysb . 2003 . 08 . 027. arXiv: hep-ph / 0306259 [hep-ph].

[27] G. F. Giudice et al. "The Strongly-Interacting Light Higgs". In: JHEP 06 (2007), p. 045. DOI: 10 .1088/1126-6708/2007/06/045. arXiv: hep-ph/ 0703164 [hep-ph]. 
[28] Giuliano Panico and Andrea Wulzer. "The Composite Nambu-Goldstone Higgs". In: Lect. Notes Phys. 913 (2016), pp.1-316. DOI: 10 . 1007 / 978 3-319-22617-0. arXiv: 1506.01961 [hep-ph].

[29] Brando Bellazzini, Csaba Csáki, and Javi Serra. "Composite Higgses". In: Eur. Phys. J. C74.5 (2014), p. 2766. DOI: 10 . $1140 /$ epjc/s10052-0142766-x. arXiv: 1401.2457 [hep-ph].

[30] Roberto Contino. "The Higgs as a Composite Nambu-Goldstone Boson". In: Physics of the large and the small, TASI 09, proceedings of the Theoretical Advanced Study Institute in Elementary Particle Physics, Boulder, Colorado, USA, 1-26 June 2009. 2011, pp. 235-306. DOI: 10 . 1142/9789814327183_ 0005. arXiv: 1005.4269 [hep-ph].

[31] Hao-Lin Li et al. "EFTs meet Higgs Nonlinearity, Compositeness and (Neutral) Naturalness". In: JHEP 09 (2019), p. 010. DOI: 10.1007/JHEP09 (2019) 010. arXiv: 1904.05359 [hep-ph].

[32] Da Liu et al. "Patterns of Strong Coupling for LHC Searches". In: JHEP 11 (2016), p. 141. DOI: 10 . 1007 / JHEP11(2016) 141. arXiv: 1603.03064 [hep-ph].

[33] Florian Goertz. "Composite Higgs theory". In: PoS ALPS2018 (2018), p. 012. DOI: $10.22323 / 1.330 .0012$. arXiv: 1812.07362 [hep-ph].

[34] Da Liu, Ian Low, and Carlos E. M. Wagner. "Modification of Higgs Couplings in Minimal Composite Models". In: Phys. Rev. D96.3 (2017), p. 035013. DOI: 10.1103/PhysRevD.96.035013. arXiv: 1703.07791 [hep-ph].

[35] Aleksandr Azatov and Jamison Galloway. "Light Custodians and Higgs Physics in Composite Models". In: Phys. Rev. D85 (2012), p. 055013. DOI: 10.1103/ PhysRevD .85.055013. arXiv: 1110.5646 [hep-ph].

[36] G. Aad et al. "Constraints on new phenomena via Higgs boson couplings and invisible decays with the ATLAS detector". In: Journal of High Energy Physics 2015 (Nov. 2015). DOI: 10.1007/JHEP11 (2015) 206. 
[37] Marcela Carena, Leandro Da Rold, and Eduardo Pontón. "Minimal Composite Higgs Models at the LHC". In: JHEP 06 (2014), p. 159. DOI: 10.1007/ JHEP06 (2014)159. arXiv: 1402.2987 [hep-ph] .

[38] Dorival Gonçalves, Tao Han, and Satyanarayan Mukhopadhyay. "Higgs Couplings at High Scales". In: Phys. Rev. D98.1 (2018), p. 015023. DOI: 10.1103/ PhysRevD .98.015023. arXiv: 1803.09751 [hep-ph].

[39] Dorival Goncalves, Tao Han, and Satyanarayan Mukhopadhyay. "Off-Shell Higgs Probe of Naturalness". In: Phys. Rev. Lett. 120.11 (2018). [Erratum: Phys. Rev. Lett.121,no.7,079902(2018)], p. 111801. DOI: 10.1103/PhysRevLett. 120 . 111801, 10.1103/PhysRevLett . 121.079902. arXiv: 1710.02149 [hep-ph].

[40] Z. Chacko, Hock-Seng Goh, and Roni Harnik. "The Twin Higgs: Natural electroweak breaking from mirror symmetry". In: Phys. Rev. Lett. 96 (2006), p. 231802. DOI: 10.1103/PhysRevLett.96.231802. arXiv: hep-ph/0506256 [hep-ph].

[41] Gustavo Burdman et al. "Colorless Top Partners, a $125 \mathrm{GeV}$ Higgs, and the Limits on Naturalness". In: Phys. Rev. D91.5 (2015), p. 055007. DOI: 10.1103/PhysRevD.91.055007. arXiv: 1411.3310 [hep-ph].

[42] Gustavo Burdman. "New solutions to the hierarchy problem". In: Braz. J. Phys. 37 (2007), pp. 506-513. DOI: $10.1590 /$ S0103-97332007000400006. arXiv: hep-ph/0703194 [hep-ph].

[43] Da Liu, Lian-Tao Wang, and Ke-Pan Xie. "Broad composite resonances and their signals at the LHC". In: Phys. Rev. D100.7 (2019), p. 075021. DOI: 10.1103/PhysRevD.100.075021. arXiv: 1901.01674 [hep-ph].

[44] Christoph Englert et al. "The $\hat{H}$-Parameter: An Oblique Higgs View". In: JHEP 09 (2019), p. 041. DOI: 10.1007 / JHEP09 (2019) 041. arXiv: 1903. 07725 [hep-ph]. 
[45] Christopher T. Hill and Elizabeth H. Simmons. "Strong Dynamics and Electroweak Symmetry Breaking". In: Phys. Rept. 381 (2003). [Erratum: Phys. Rept.390,553(2004)], pp. 235-402. DOI: 10.1016/S0370-1573(03) 00140-6. arXiv: hep-ph/0203079 [hep-ph].

[46] Csaba Csáki, Salvator Lombardo, and Ofri Telem. "TASI Lectures on Nonsupersymmetric BSM Models". In: Proceedings, Theoretical Advanced Study Institute in Elementary Particle Physics : Anticipating the Next Discoveries in Particle Physics (TASI 2016): Boulder, CO, USA, June 6-July 1, 2016. WSP. WSP, 2018, pp. 501-570. DOI: 10.1142/9789813233348_0007. arXiv: 1811.04279 [hep-ph]. URL: http://webtheory.sns.it/ggilectures2018/ csaki/notes.pdf.

[47] Csaba Csáki and Philip Tanedo. "Beyond the Standard Model". In: Proceedings, 2013 European School of High-Energy Physics (ESHEP 2013): Paradfurdo, Hungary, June 5-18, 2013. 2015, pp. 169-268. DOI: 10.5170/CERN2015-004.169. arXiv: 1602.04228 [hep-ph].

[48] Csaba Csaki, Christophe Grojean, and John Terning. "Alternatives to an Elementary Higgs". In: Rev. Mod. Phys. 88.4 (2016), p. 045001. DOI: 10 . 1103/RevModPhys.88.045001. arXiv: 1512.00468 [hep-ph] .

[49] R. Hofstadter and R. W. McAllister. "Electron Scattering From the Proton". In: Phys. Rev. 98 (1955), pp. 217-218. DOI: 10.1103/PhysRev.98.217.

[50] Vardan Khachatryan et al. "Measurement and QCD analysis of double-differential inclusive jet cross sections in pp collisions at $\sqrt{s}=8 \mathrm{TeV}$ and cross section ratios to 2.76 and 7 TeV". In: JHEP 03 (2017), p. 156. DOI: 10.1007 / JHEP03 (2017) 156. arXiv: 1609.05331 [hep-ex].

[51] Eduardo de Rafael. "An Introduction to sum rules in QCD: Course". In: Probing the standard model of particle interactions. Proceedings, Summer School in Theoretical Physics, NATO Advanced Study Institute, 68th session, 
Les Houches, France, July 28-September 5, 1997. Pt. 1, 2. 1997, pp. 11711218. arXiv: hep-ph/9802448 [hep-ph].

[52] Steven Weinberg. "Precise relations between the spectra of vector and axial vector mesons". In: Phys. Rev. Lett. 18 (1967), pp. 507-509. DOI: 10.1103/ PhysRevLett.18.507.

[53] J.J. Sakurai. "Theory of strong interactions". In: Annals Phys. 11 (1960), pp. 1-48. DOI: 10.1016/0003-4916(60)90126-3.

[54] Masako Bando, Taichiro Kugo, and Koichi Yamawaki. "Nonlinear Realization and Hidden Local Symmetries". In: Phys. Rept. 164 (1988), pp. 217-314. DOI: $10.1016 / 0370-1573(88) 90019-1$.

[55] OTTO STERN. "The method of molecular rays". In: Nobel Lectures, Physics 1942-1961 (1946), pp. 8-16.

[56] G. Breit and I. I. Rabi. "On the Interpretation of Present Values of Nuclear Moments". In: Phys. Rev. 46 (3 1934), pp. 230-231. DOI: 10.1103/PhysRev. 46.230. URL: https://link.aps.org/doi/10.1103/PhysRev.46.230.

[57] Gerard 't Hooft. "A Planar Diagram Theory for Strong Interactions". In: Nucl. Phys. B72 (1974). [,337(1973)], p. 461. DOI: 10.1016/0550-3213(74) 901540.

[58] Sidney R. Coleman, J. Wess, and Bruno Zumino. "Structure of phenomenological Lagrangians. 1." In: Phys. Rev. 177 (1969), pp. 2239-2247. DOI: 10. 1103/PhysRev.177.2239.

[59] Curtis G. Callan Jr. et al. "Structure of phenomenological Lagrangians. 2." In: Phys. Rev. 177 (1969), pp. 2247-2250. DOI: 10.1103/PhysRev.177.2247.

[60] Edward Witten. "Baryons in the 1/n Expansion". In: Nucl. Phys. B160 (1979), pp. 57-115. DOI: 10.1016/0550-3213(79)90232-3. 
[61] Gunnar Kallen. "On the definition of the Renormalization Constants in Quantum Electrodynamics". In: Helv. Phys. Acta 25.4 (1952). [,509(1952)], p. 417. DOI: $10.1007 / 978-3-319-00627-7 \_90$.

[62] Martin Breidenbach et al. "Observed Behavior of Highly Inelastic electronProton Scattering". In: Phys. Rev. Lett. 23 (1969), pp. 935-939. DOI: 10. 1103/PhysRevLett. 23.935.

[63] Richard P. Feynman. "Very high-energy collisions of hadrons". In: Phys. Rev. Lett. 23 (1969). [,494(1969)], pp. 1415-1417. DOI: 10 .1103/PhysRevLett . 23.1415 .

[64] Elliott D. Bloom et al. "High-Energy Inelastic e p Scattering at 6-Degrees and 10-Degrees". In: Phys. Rev. Lett. 23 (1969), pp. 930-934. DoI: 10.1103/ PhysRevLett.23.930.

[65] J. R. Pelaez. "From controversy to precision on the sigma meson: a review on the status of the non-ordinary $f_{0}(500)$ resonance". In: Phys. Rept. 658 (2016), p. 1. DOI: 10.1016/j .physrep.2016.09.001. arXiv: 1510.00653 [hep-ph].

[66] Lisa Randall and Raman Sundrum. "A Large mass hierarchy from a small extra dimension". In: Phys. Rev. Lett. 83 (1999), pp. 3370-3373. DOI: 10 . 1103/PhysRevLett.83.3370. arXiv: hep-ph/9905221 [hep-ph].

[67] Nima Arkani-Hamed, Massimo Porrati, and Lisa Randall. "Holography and phenomenology". In: JHEP 08 (2001), p. 017. DOI: 10.1088/1126-6708/ 2001/08/017. arXiv: hep-th/0012148 [hep-th] .

[68] R. Rattazzi and A. Zaffaroni. "Comments on the holographic picture of the Randall-Sundrum model". In: JHEP 04 (2001), p. 021. DOI: 10.1088/11266708/2001/04/021. arXiv: hep-th/0012248 [hep-th] .

[69] Raman Sundrum. "Tasi 2004 lectures: To the fifth dimension and back". In: Theoretical Advanced Study Institute in Elementary Particle Physics: Many Dimensions of String Theory (TASI 2005) Boulder, Colorado, June 5-July 1, 2005. [,585(2005)]. 2005, pp. 585-630. arXiv: hep-th/0508134 [hep-th]. 
[70] Juan Martin Maldacena. "The Large N limit of superconformal field theories and supergravity". In: Int. J. Theor. Phys. 38 (1999). [Adv. Theor. Math. Phys.2,231(1998)], pp. 1113-1133. DOI: 10 . 1023/A : 1026654312961, 10 . 4310/ATMP. 1998.v2.n2.a1. arXiv: hep-th/9711200 [hep-th].

[71] Steven Weinberg. The Quantum theory of fields. Vol. 1: Foundations. Cambridge University Press, 2005. ISBN: 9780521670531, 9780511252044.

[72] Steven Weinberg. The quantum theory of fields. Vol. 2: Modern applications. Cambridge University Press, 2013. ISBN: 9781139632478, 9780521670548, 9780521550024.

[73] Michael E. Peskin and Daniel V. Schroeder. An Introduction to quantum field theory. Reading, USA: Addison-Wesley, 1995. ISBN: 9780201503975, 0201503972. URL: http://www.slac.stanford.edu/ mpeskin/QFT.html.

[74] Matthew D. Schwartz. Quantum Field Theory and the Standard Model. Cambridge University Press, 2014. ISBN: 1107034736, 9781107034730. URL: http: //www . cambridge .org/us/academic/subjects/physics/theoretical physics - and - mathematical - physics / quantum - field - theory - and standard-model.

[75] Chris Quigg. Gauge Theories of the Strong, Weak, and Electromagnetic Interactions. USA: Princeton University Press, 2013. ISBN: 9780691135489, 9781400848225. URL: http://chrisquigg.com/gauge-theories/.

[76] Anthony Duncan. "The Conceptual Framework of Quantum Field Theory". In: The Conceptual Framework of Quantum Field Theory (Jan. 2013). DOI: 10.1093/acprof : oso/9780199573264.001.0001.

[77] J. F. Donoghue, E. Golowich, and Barry R. Holstein. "Dynamics of the standard model". In: Camb. Monogr. Part. Phys. Nucl. Phys. Cosmol. 2 (1992). [Camb. Monogr. Part. Phys. Nucl. Phys. Cosmol.35(2014)], pp. 1-540. DOI: $10.1017 / \mathrm{CB} 09780511524370$.

[78] Vernon D. Barger and R. J. N. Phillips. COLLIDER PHYSICS. 1987. ISBN: 9780201149456. 
[79] Howard Georgi. "Unparticle physics". In: Phys. Rev. Lett. 98 (2007), p. 221601. DOI: 10.1103/PhysRevLett.98.221601. arXiv: hep-ph/0703260 [hep-ph].

[80] Howard Georgi. "Another odd thing about unparticle physics". In: Phys. Lett. B650 (2007), pp. 275-278. DOI: 10.1016/j . physletb.2007.05.037. arXiv: 0704.2457 [hep-ph].

[81] David Simmons-Duffin. "The Conformal Bootstrap". In: Proceedings, Theoretical Advanced Study Institute in Elementary Particle Physics: New Frontiers in Fields and Strings (TASI 2015): Boulder, CO, USA, June 1-26, 2015. 2017, pp. 1-74. DOI: 10 . 1142/9789813149441_0001. arXiv: 1602.07982 [hep-th].

[82] David Poland, Slava Rychkov, and Alessandro Vichi. "The Conformal Bootstrap: Theory, Numerical Techniques, and Applications". In: Rev. Mod. Phys. 91 (2019), p. 015002. DOI: 10.1103/RevModPhys.91.015002. arXiv: 1805. 04405 [hep-th].

[83] Riccardo Rattazzi et al. "Bounding scalar operator dimensions in 4D CFT". In: JHEP 12 (2008), p. 031. DOI: 10.1088/1126-6708/2008/12/031. arXiv: 0807.0004 [hep-th].

[84] Roman Zwicky. "A brief Introduction to Dispersion Relations and Analyticity". In: Proceedings, Quantum Field Theory at the Limits: from Strong Fields to Heavy Quarks (HQ 2016): Dubna, Russia, July 18-30, 2016. 2017, pp. 93-120. DOI: 10.3204/DESY-PROC-2016-04/Zwicky. arXiv: 1610.06090 [hep-ph].

[85] Frank Wilczek. "Origins of Mass". In: Central Eur. J. Phys. 10 (2012), pp. 10211037. DOI: $10.2478 / \mathrm{s} 11534-012-0121-0$. arXiv: 1206.7114 [hep-ph].

[86] James D. Wells. "The theoretical physics ecosystem behind the discovery of the Higgs boson". In: (2016). arXiv: 1609.04268 [physics.hist-ph]. 
[87] Gian Francesco Giudice. "The Dawn of the Post-Naturalness Era". In: From My Vast Repertoire ...: Guido Altarelli's Legacy. Ed. by Aharon Levy, Stefano Forte, and Giovanni Ridolfi. 2019, pp. 267-292. DOI: 10.1142/9789813238053_ 0013. arXiv: 1710.07663 [physics.hist-ph].

[88] Chris Quigg. "Unanswered Questions in the Electroweak Theory". In: Ann. Rev. Nucl. Part. Sci. 59 (2009), pp. 505-555. DOI: 10.1146/annurev.nucl. 010909.083126. arXiv: 0905.3187 [hep-ph].

[89] H. Georgi. "Why I would be very sad if a Higgs boson were discovered". In: (1993), pp. 337-342.

[90] D. de Florian et al. "Handbook of LHC Higgs Cross Sections: 4. Deciphering the Nature of the Higgs Sector". In: (2016). DOI: 10 . 2172/1345634 , 10 . 23731/CYRM-2017-002. arXiv: 1610.07922 [hep-ph].

[91] Nikolas Kauer and Giampiero Passarino. "Inadequacy of zero-width approximation for a light Higgs boson signal". In: JHEP 08 (2012), p. 116. DOI: 10.1007/JHEP08(2012)116. arXiv: 1206.4803 [hep-ph].

[92] Tao Han. "Collider phenomenology: Basic knowledge and techniques". In: Physics in $D>=4$. Proceedings, Theoretical Advanced Study Institute in elementary particle physics, TASI 2004, Boulder, USA, June 6-July 2, 2004. 2005, pp. 407-454. DOI: 10 . 1142 /9789812773579_0008. arXiv: hep-ph / 0508097 [hep-ph].

[93] Dorival Goncalves, Tilman Plehn, and Jennifer M. Thompson. "Weak boson fusion at 100 TeV". In: Phys. Rev. D95.9 (2017), p. 095011. DOI: 10.1103/ PhysRevD.95.095011. arXiv: 1702.05098 [hep-ph].

[94] Aleksandr Azatov et al. "Taming the off-shell Higgs boson". In: Zh. Eksp. Teor. Fiz. 147 (2015). [J. Exp. Theor. Phys.120,354(2015)], pp. 410-425. DOI: 10.1134/S1063776115030140,10.7868/S0044451015030039. arXiv: 1406 . 6338 [hep-ph] 
[95] Nima Arkani-Hamed et al. "Physics opportunities of a $100 \mathrm{TeV}$ proton-proton collider". In: Phys. Rept. 652 (2016), pp. 1-49. DOI: 10.1016/j · physrep. 2016.07.004. arXiv: 1511.06495 [hep-ph].

[96] Roberto Contino et al. "Strong Double Higgs Production at the LHC". In: JHEP 05 (2010), p. 089. DOI: 10.1007/JHEP05 (2010) 089. arXiv: 1002.1011 [hep-ph].

[97] J. Alison et al. "Higgs Boson Pair Production at Colliders: Status and Perspectives". In: Double Higgs Production at Colliders Batavia, IL, USA, September 4, 2018-9, 2019. Ed. by B. Di Micco et al. 2019. arXiv: 1910.00012 [hep-ph]. URL: https://lss . fnal gov/archive/2019/conf/fermilabconf-19-468-e-t.pdf.

[98] Celine Degrande et al. "UFO - The Universal FeynRules Output". In: Comput. Phys. Commun. 183 (2012), pp. 1201-1214. DOI: 10.1016/j.cpc.2012.01. 022. arXiv: 1108.2040 [hep-ph].

[99] Adam Alloul et al. "FeynRules 2.0 - A complete toolbox for tree-level phenomenology". In: Comput. Phys. Commun. 185 (2014), pp. 2250-2300. DOI: 10.1016/j.cpc.2014.04.012. arXiv: 1310.1921 [hep-ph].

[100] Eric Conte, Benjamin Fuks, and Guillaume Serret. "MadAnalysis 5, A UserFriendly Framework for Collider Phenomenology". In: Comput. Phys. Commun. 184 (2013), pp. 222-256. DOI: 10.1016/ j.cpc.2012.09.009. arXiv: 1206.1599 [hep-ph].

[101] T. Hahn and M. Perez-Victoria. "Automatized one loop calculations in fourdimensions and D-dimensions". In: Comput. Phys. Commun. 118 (1999), pp. 153-165. DOI: 10 . 1016/S0010-4655(98) 00173-8. arXiv: hep-ph / 9807565 [hep-ph]. 
[102] J. Alwall et al. "The automated computation of tree-level and next-to-leading order differential cross sections, and their matching to parton shower simulations". In: JHEP 07 (2014), p. 079. DOI: 10.1007/JHEP07 (2014) 079. arXiv: 1405.0301 [hep-ph].

[103] Priscila de Aquino et al. "ALOHA: Automatic Libraries Of Helicity Amplitudes for Feynman Diagram Computations". In: Comput. Phys. Commun. 183 (2012), pp. 2254-2263. DOI: $10.1016 /$ j . cpc . 2012 . 05.004 . arXiv: 1108.2041 [hep-ph]. 